Supplement of Geosci. Model Dev., 13, 5875-5896, 2020

https://doi.org/10.5194/gmd-13-5875-2020-supplement

(C) Author(s) 2020. This work is distributed under

the Creative Commons Attribution 4.0 License.

(c) (i)

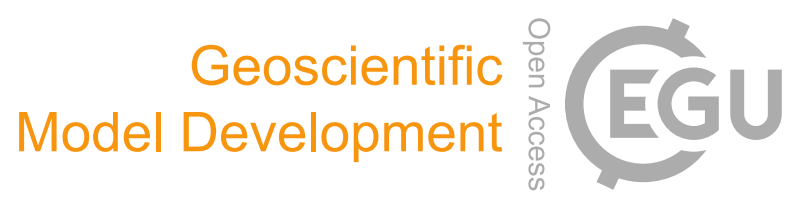

Supplement of

\title{
Implementation of the RCIP scheme and its performance for 1-D age computations in ice-sheet models
}

Fuyuki Saito et al.

Correspondence to: Fuyuki Saito (saitofuyuki@jamstec.go.jp)

The copyright of individual parts of the supplement might differ from the CC BY 4.0 License. 


\section{S1 Comprehensive experiment sheets}

In addition to the experiments presented in the main paper, several experimental configuration combinations of parameters are examined. In this Supplement, some representative results of them are provided. All of the experiments shown are examined under non-steady surface mass balance configurations. Each page contains two figures: the upper one is the result obtained using a square-wave type surface mass balance input, while the lower is one with the same configuration except for applying a cosine-wave type input. One figure contains five sub-figures. (a) profiles of the computed age, $(b, d)$ The computed age differences relative to the result of the RCIP+corr case, and (c,e) the annual layer thickness. The upper sub-figures (a-c) show the profiles in terms of depths, while the lower sub-figures (d-e) show them in terms of the computed ages. The surface mass balance input used in each experiment is shown in the lower left figure for only first 200 or $100 \mathrm{kyr}$. The prescribed time evolution of ice-thickness is also shown in the lower left figure for the non-steady thickness experiments. The grey and black lines in $(\mathrm{a}, \mathrm{c}, \mathrm{e})$ correspond to 'benchmark' profiles which are computed using constant surface/basal mass balance and thickness.

Figures $\mathrm{S} 1$ to $\mathrm{S6}$ are the results of a most standard experiments: constant $H=3000 \mathrm{~m}$, $a_{\mathrm{H}}, a_{\mathrm{L}}=3,1.5 \mathrm{~cm} \mathrm{yr}^{-1}, P_{\mathrm{T}}=100 \mathrm{kyr}, P_{\mathrm{H}}: P_{\mathrm{L}}=1: 1$, and the uniform discretization of 129 levels (see Eq 57 in the main text about definition of $a_{\mathrm{H}}, a_{\mathrm{L}}, P_{\mathrm{T}}, P_{\mathrm{H}}$ and $P_{\mathrm{L}}$ ). Basal mass balances are set as constant $M_{\mathrm{b}}=0 \mathrm{~mm} \mathrm{yr}^{-1}$ S1S2, $M_{\mathrm{b}}=0.3 \mathrm{~mm} \mathrm{yr}^{-1}$ S3 S4 , and $M_{\mathrm{b}}=3 \mathrm{~mm} \mathrm{yr}^{-1}$ (S556). All of the results are the snapshots at $t=1 \mathrm{Myr}$.

Figures $\mathrm{S} 7$ to $\mathrm{S} 12$ are the same combination as presented above except for $a_{\mathrm{L}}=0.75 \mathrm{~cm} \mathrm{yr}^{-1}$. Figures S13 to S18 are the same except for $a_{\mathrm{L}}=0 \mathrm{~cm} \mathrm{yr}^{-1}$. Figures S19 to S22 are the same except for $a_{\mathrm{L}}=-15 \mathrm{~cm} \mathrm{yr}^{-1}$.

Two types of sensitivity experiments regarding to the shape of prescribed time-evolution of surface mass balance inputs are performed: one type obtained using a different $P_{\mathrm{H}}: P_{\mathrm{L}}$, and the other using a different $P_{\mathrm{T}}$. Figures $\mathrm{S} 23$ to $\mathrm{S} 26$ are the results obtained using a longer $P_{\mathrm{H}}$, as $P_{\mathrm{H}}: P_{\mathrm{L}}=7: 1$, and Figures $\mathrm{S} 27$ to $\mathrm{S} 30$ are the results using a shorter $P_{\mathrm{H}}$, as $P_{\mathrm{H}}: P_{\mathrm{L}}=1: 7$. Figures $\mathrm{S} 31$ to $\mathrm{S38}$ are the results obtained using $P_{\mathrm{T}}=50 \mathrm{kyr}$, Figures $\mathrm{S39}$ to $\mathrm{S} 46$ using $P_{\mathrm{T}}=$ $20 \mathrm{kyr}$, and Figures $\mathrm{S} 47$ to $\$ 54$ using $P_{\mathrm{T}}=10 \mathrm{kyr}$.

Two types of sensitivity experiments regarding to the shape of prescribed time evolution of ice-thickness are performed: one type with $\tau_{H}=10 \mathrm{kyr}$ (Figs S55 to S58), and the other with $\tau_{H}=3 \mathrm{kyr}$ (Figs S59 to S62). (see Eq61 in the main text about definition of $\tau_{H}$ ).

The series of experiments obtained using constant $H=3000 \mathrm{~m}$ and $M_{\mathrm{b}}=0 \mathrm{~mm} \mathrm{yr}^{-1}$ are repeated by higher resolution configurations. Figures $S 63$ to $S 70$ are the results of $P_{\mathrm{T}}=100 \mathrm{kyr}$; Figures S71 to S94 are results obtained using a uniform discretization of 513 levels. Figures S95 to 5126 are the same results but using a smooth non-uniform discretization of 513 levels. Figures $\mathrm{S} 127$ to $\mathrm{S} 158$ are the same results but using a non-smooth non-uniform discretization of 477 levels. The snapshots at $t=2 \mathrm{Myr}$ are plotted for those experiment with higher resolutions. Designs of these higher resolution discretization are shown in Fig. 14 in the main text.

Furthermore, the series of experiments obtained using constant $H=3000 \mathrm{~m}$ and $M_{\mathrm{b}}=$ $0 \mathrm{~mm} \mathrm{yr}^{-1}$ are repeated by lower resolution configurations. Figures $\mathrm{S159}$ to $\mathrm{S166}$ are the results of $P_{\mathrm{T}}=100 \mathrm{kyr}$, obtained using a uniform discretization of 33 levels. 

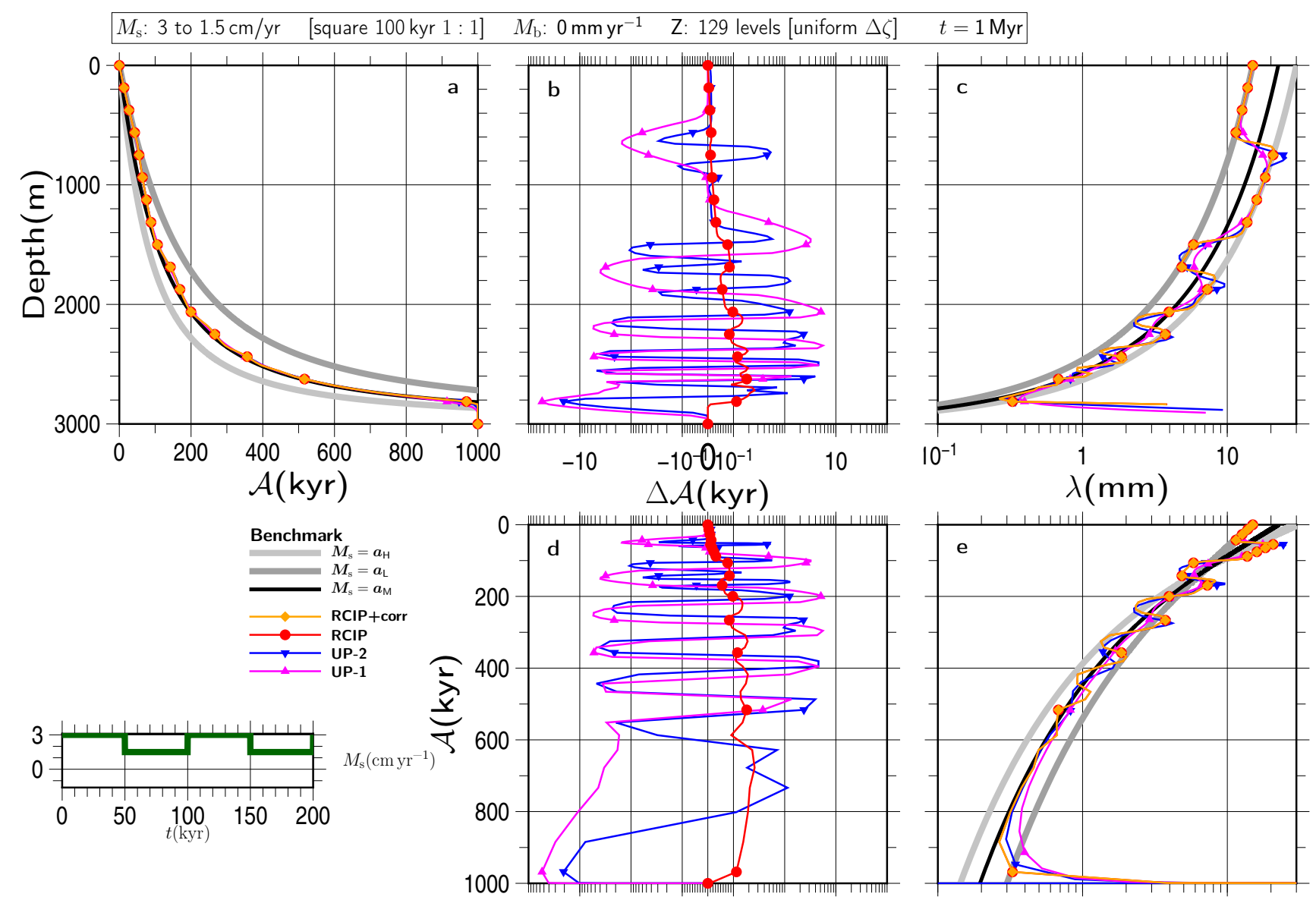

Figure S1
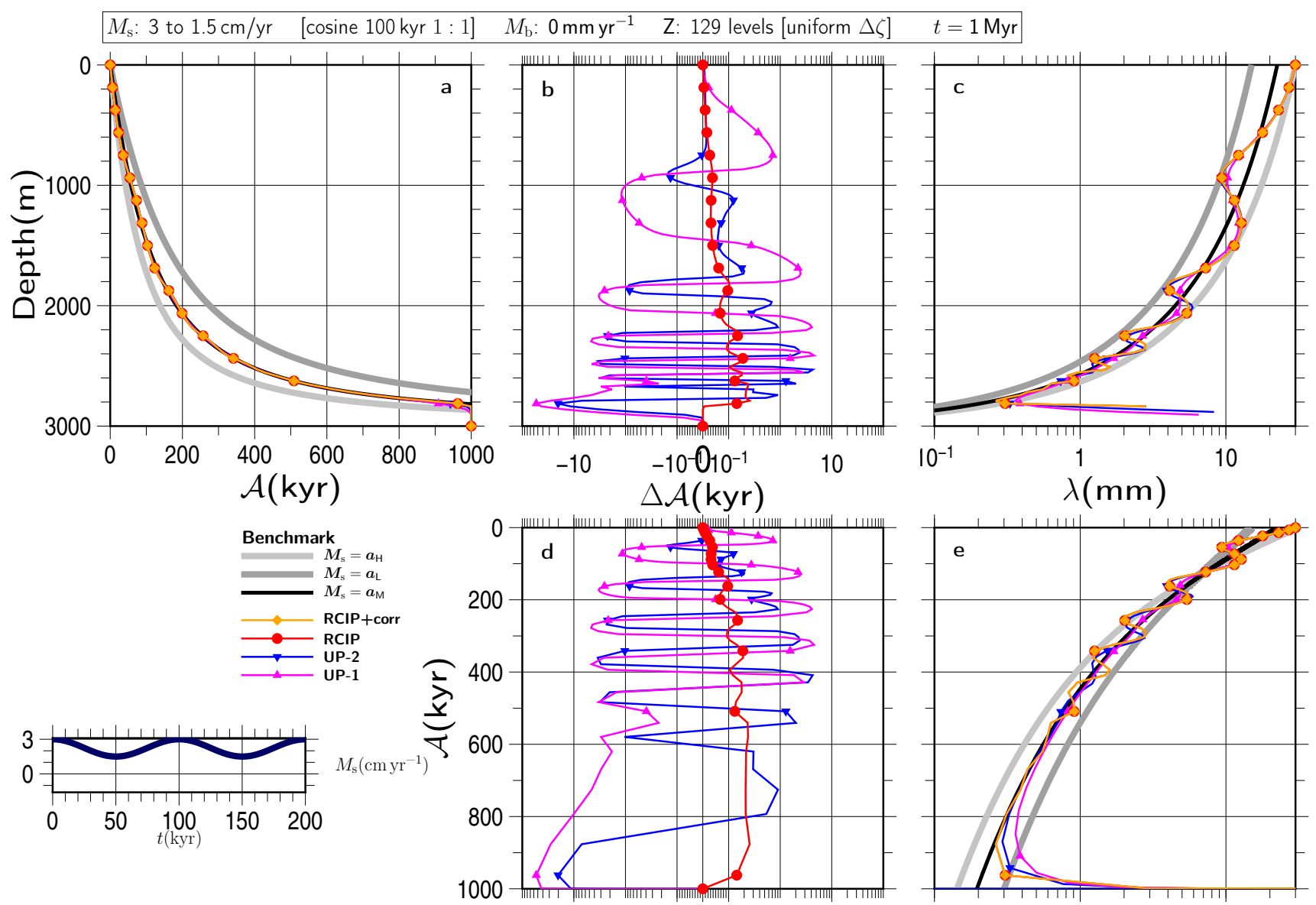

Figure S2 

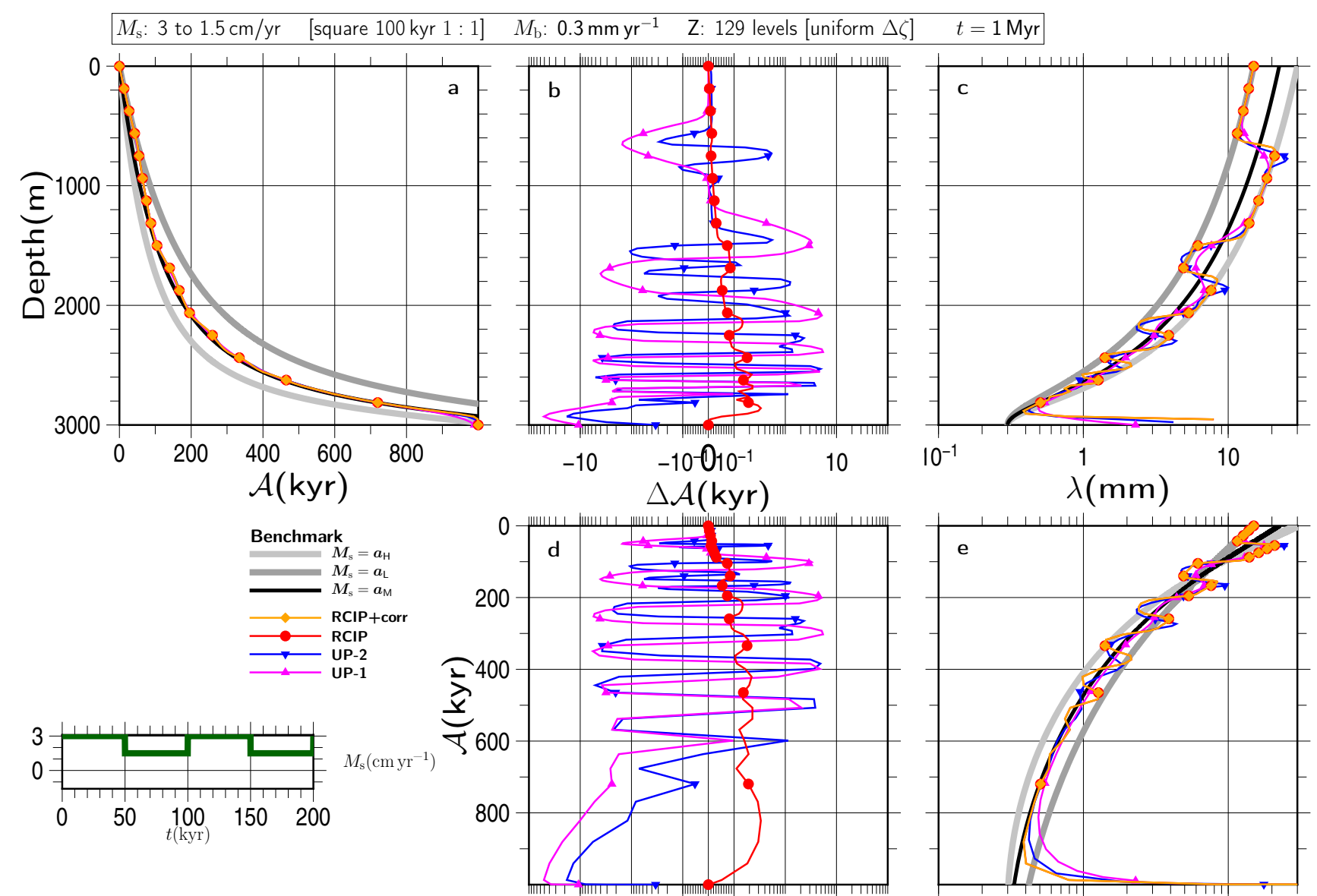

Figure S3
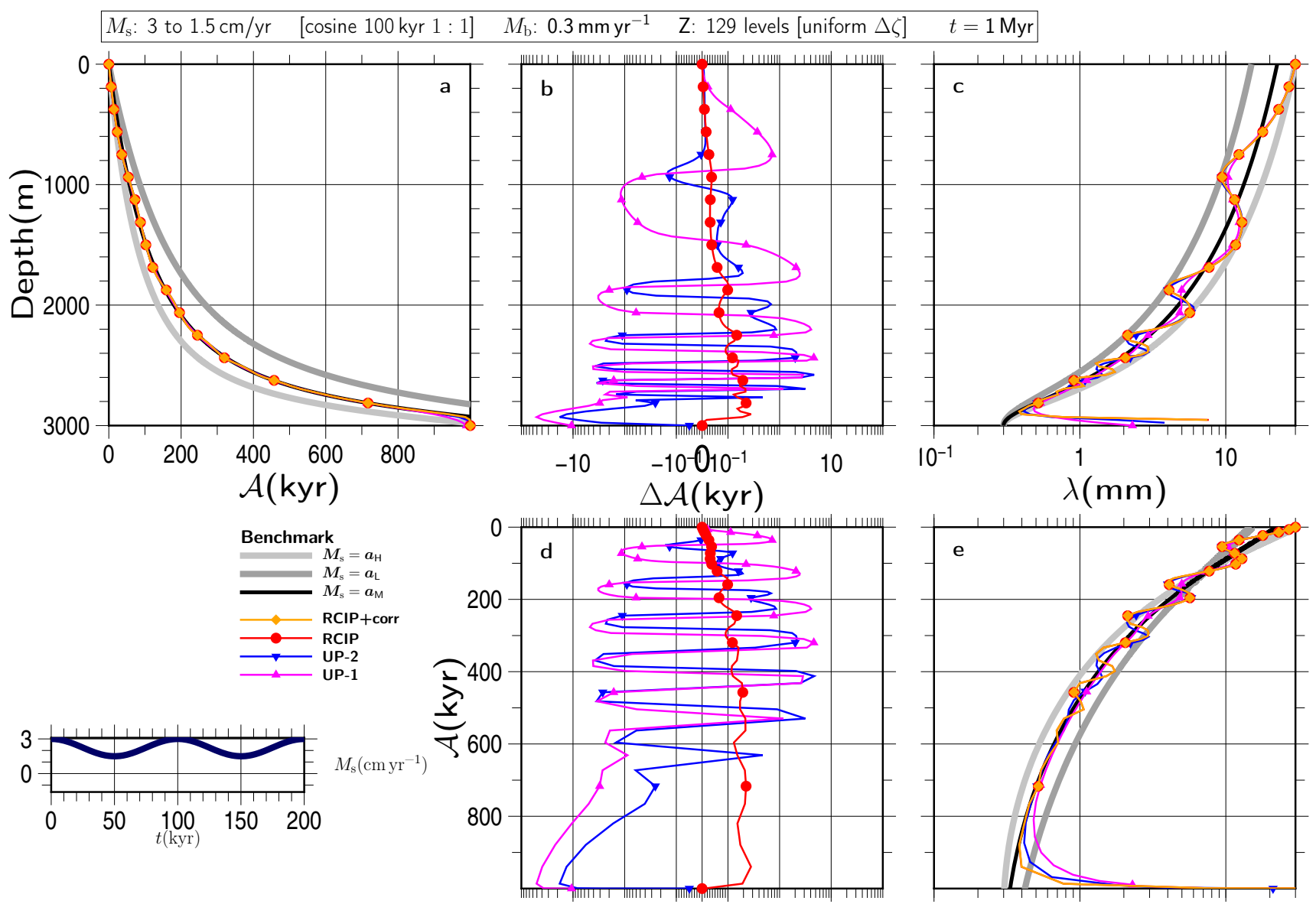

Figure S4 

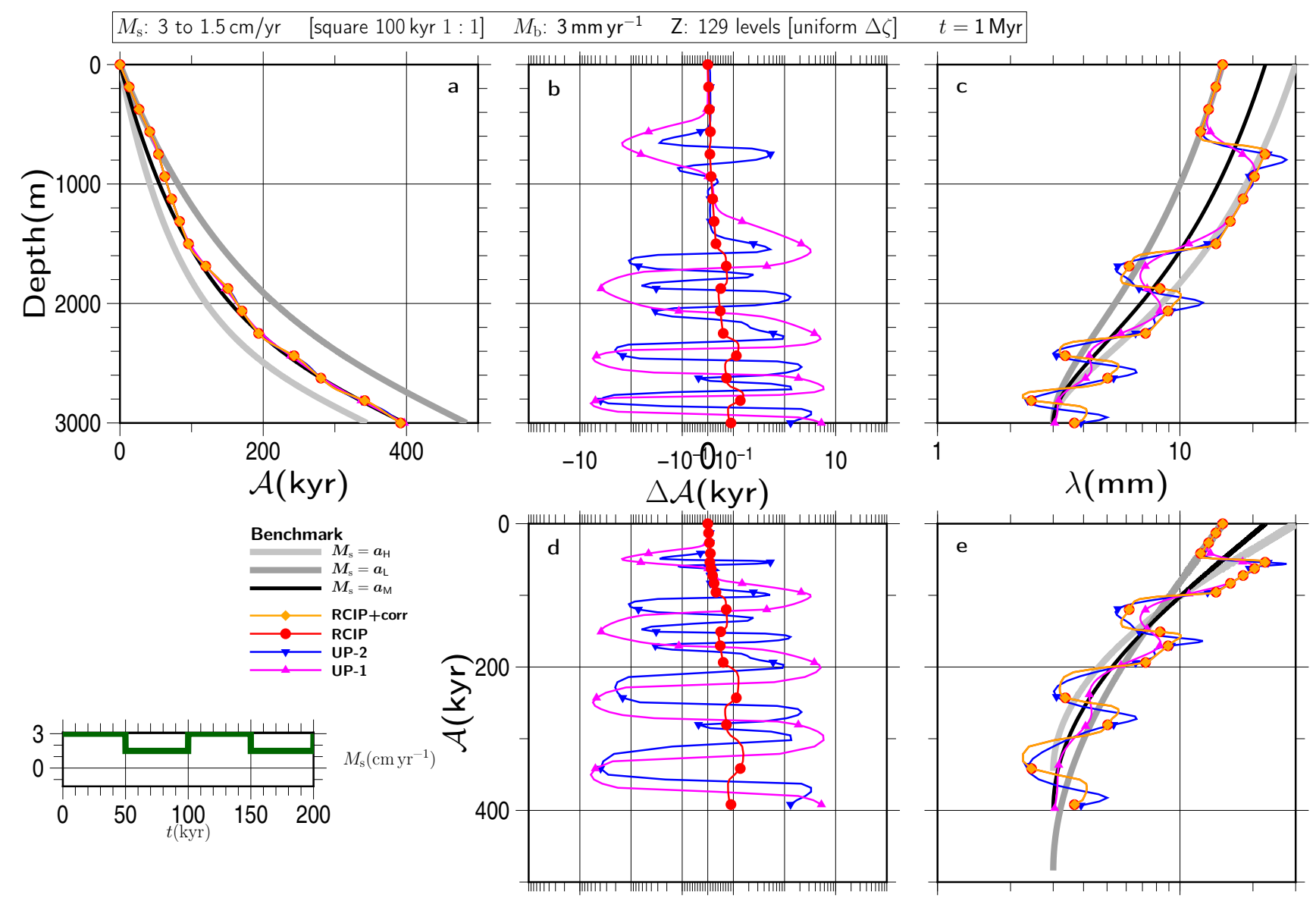

Figure S5
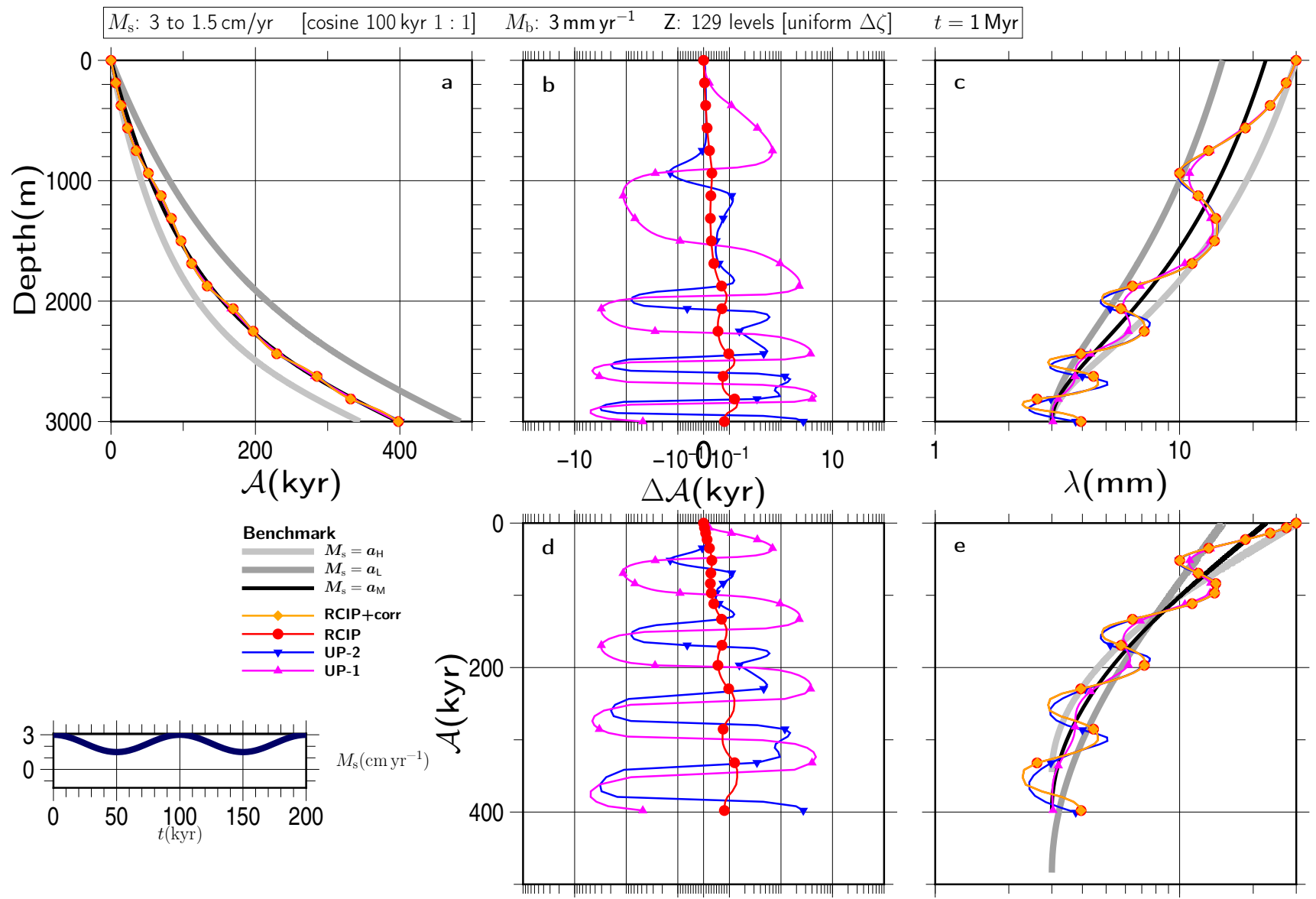

Figure S6 

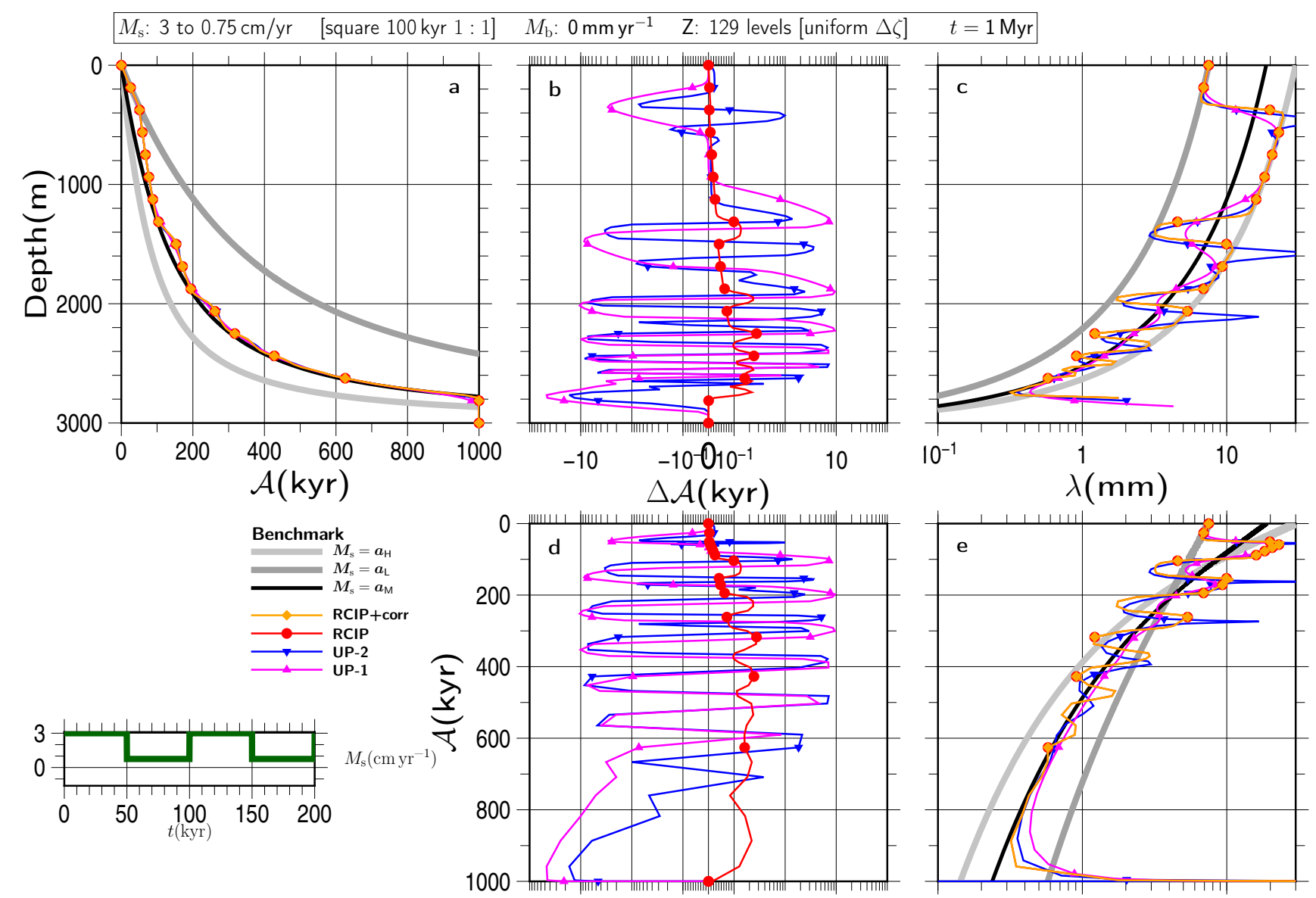

Figure S7
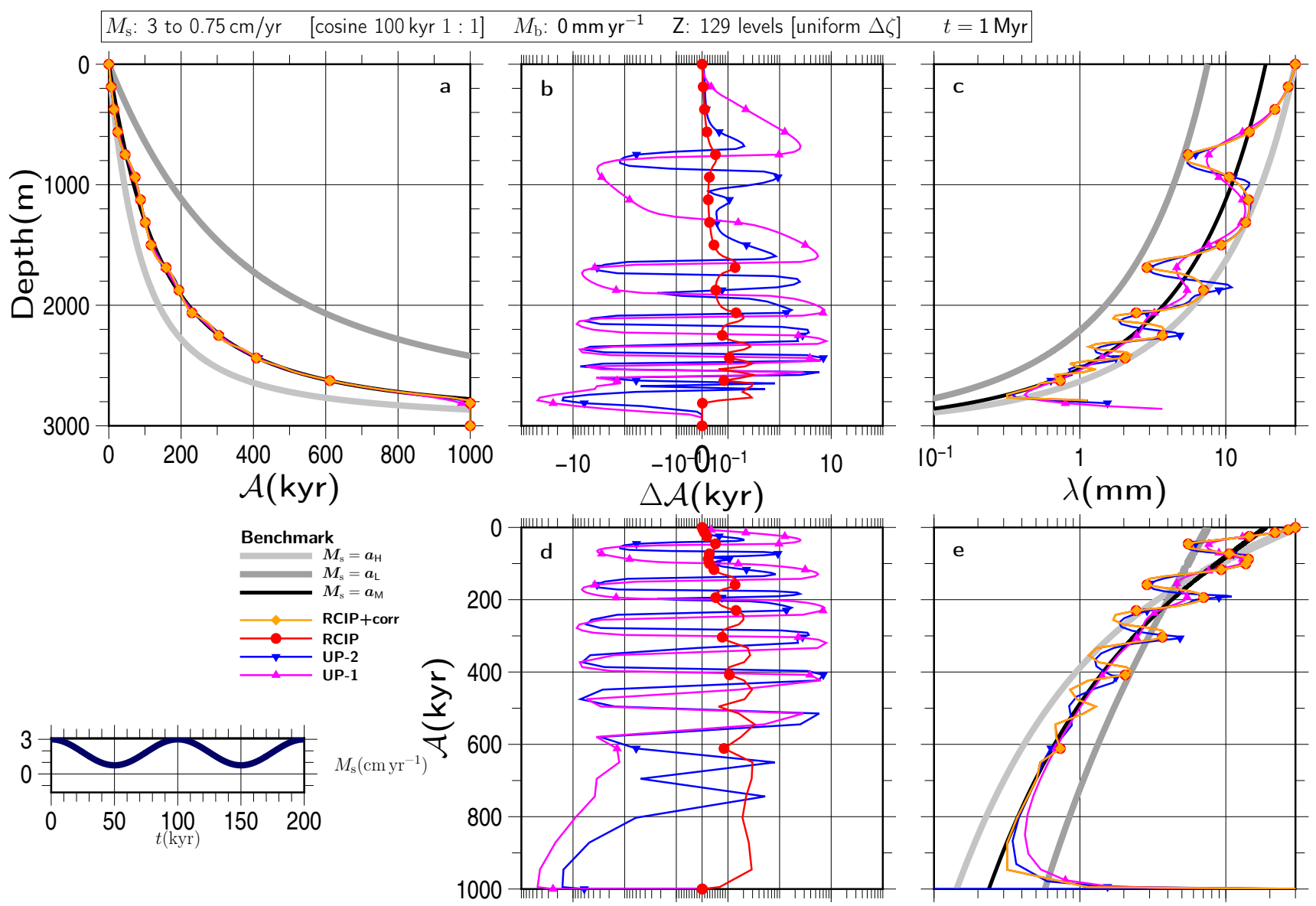

Figure S8 

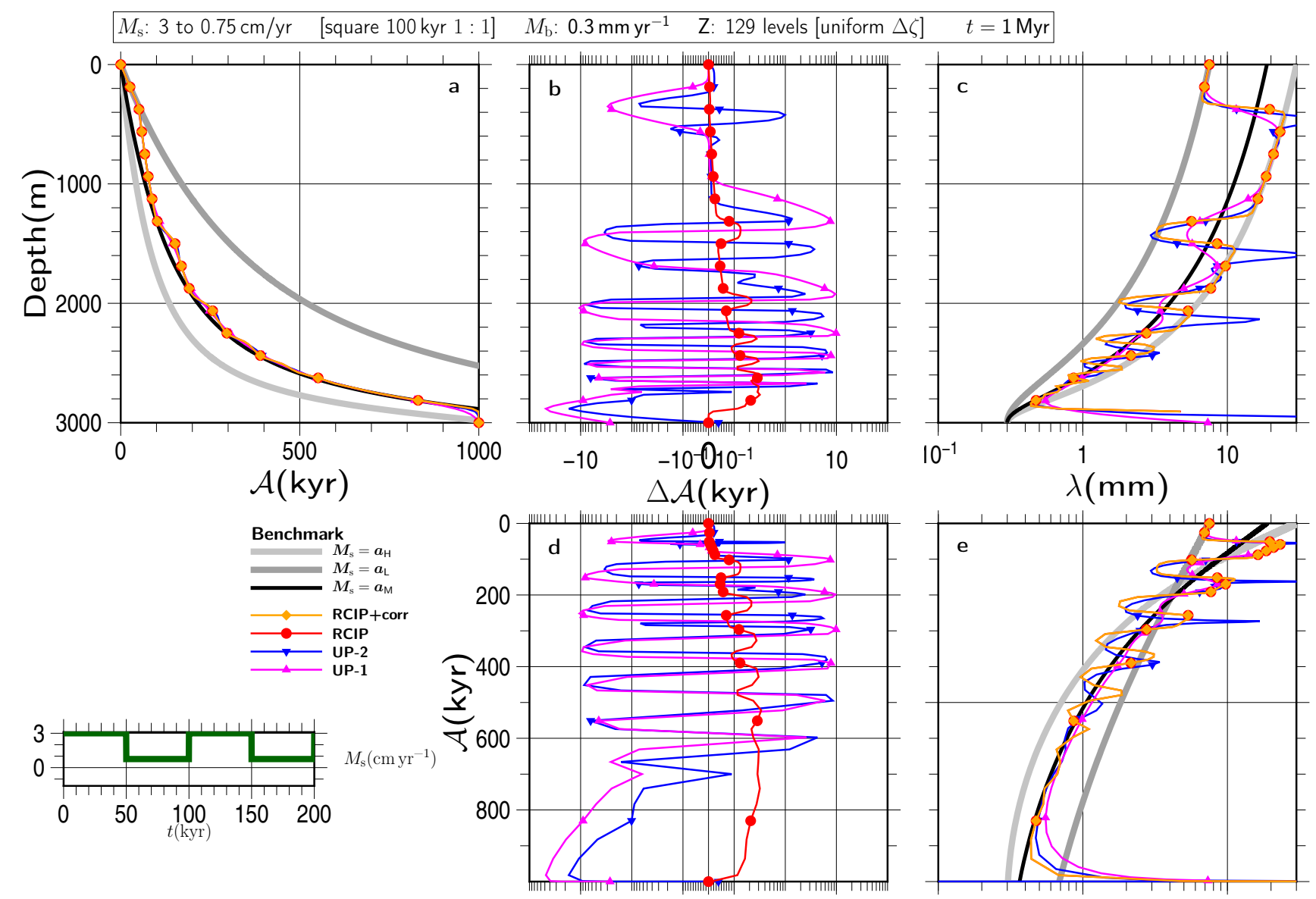

Figure S9
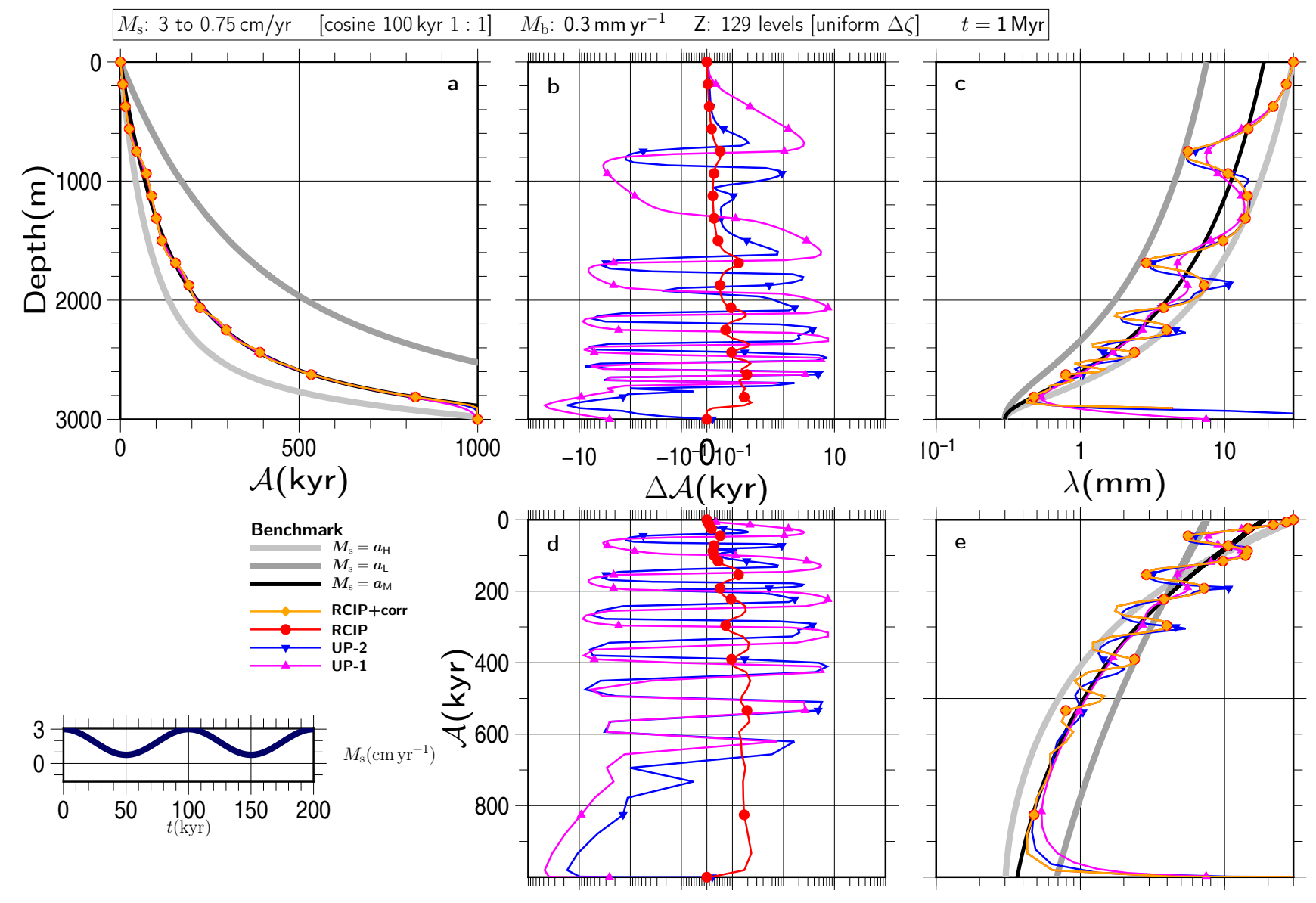

Figure S10 

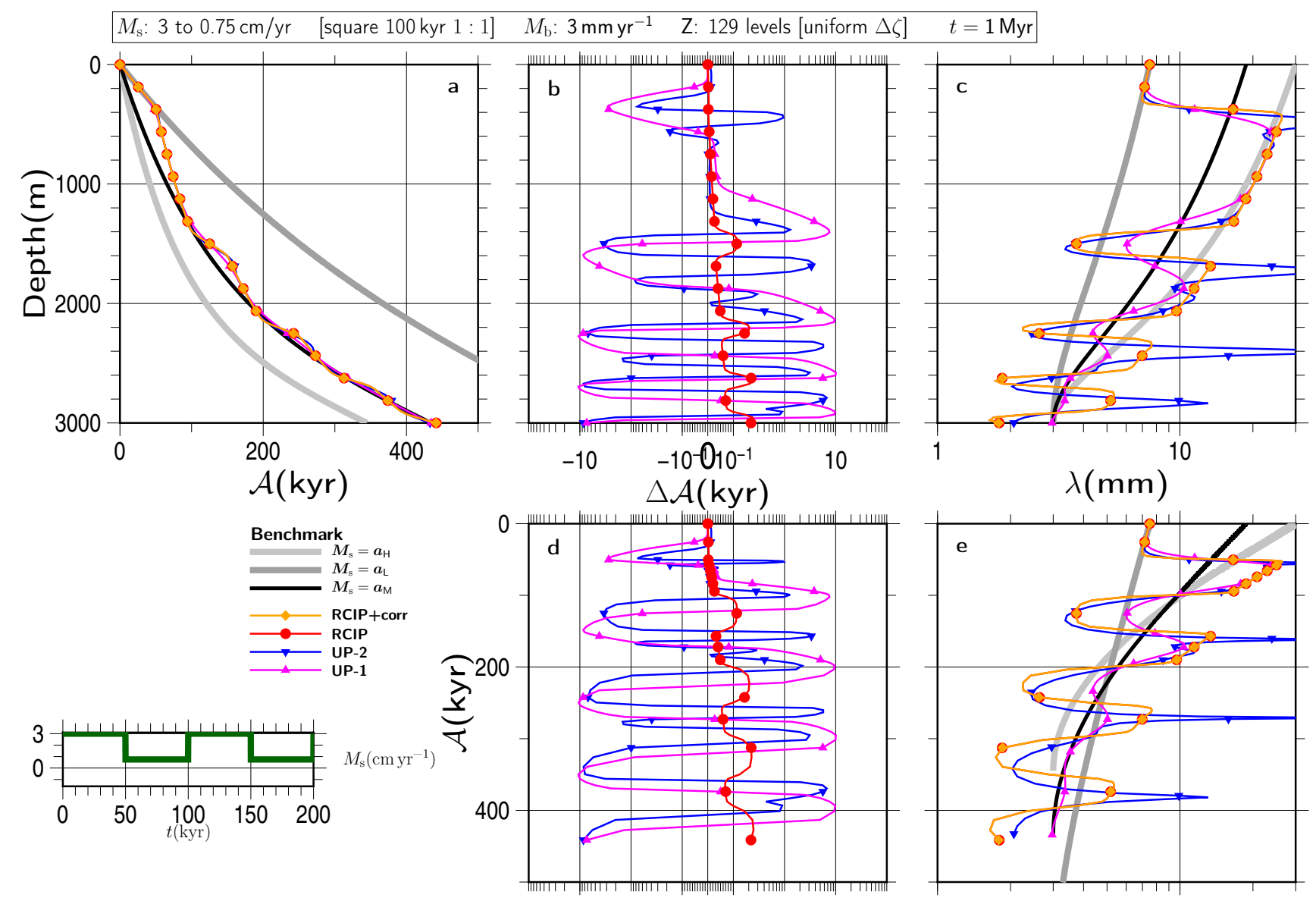

Figure S11
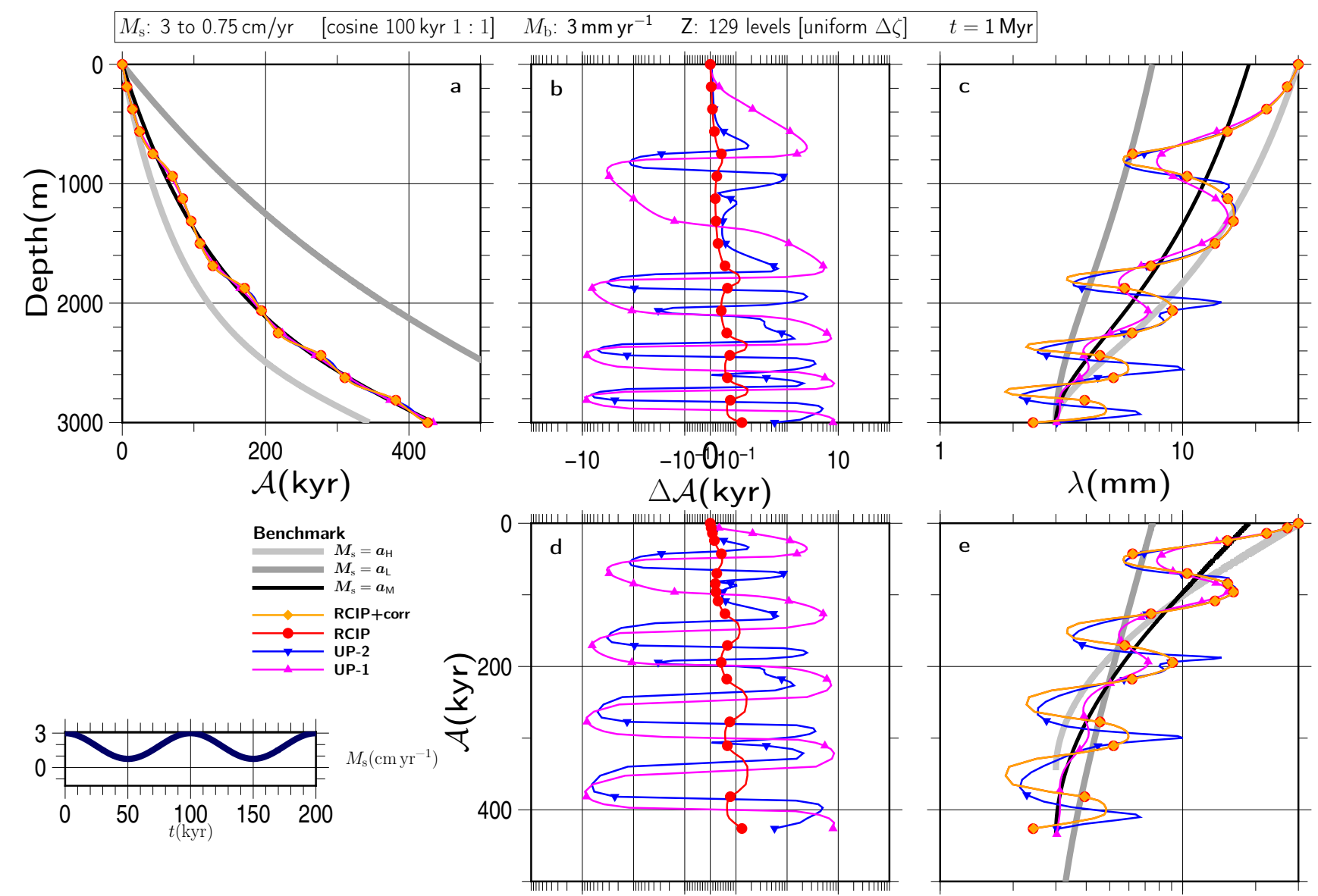

Figure S12 

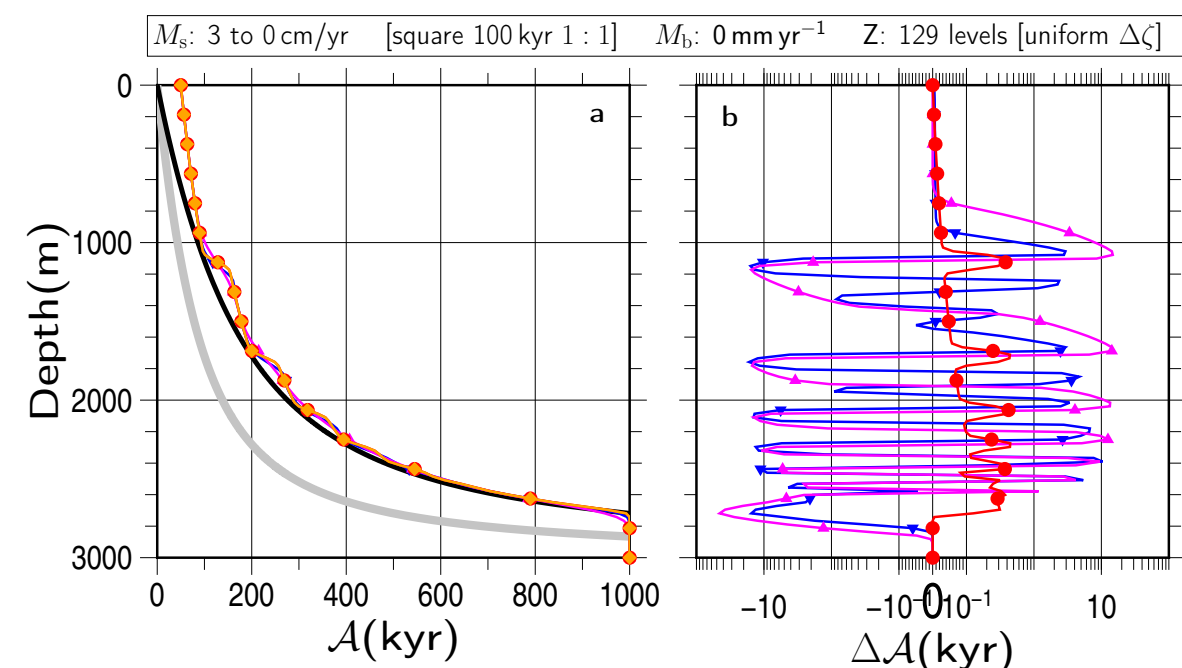

$t=1 \mathrm{Myr}$
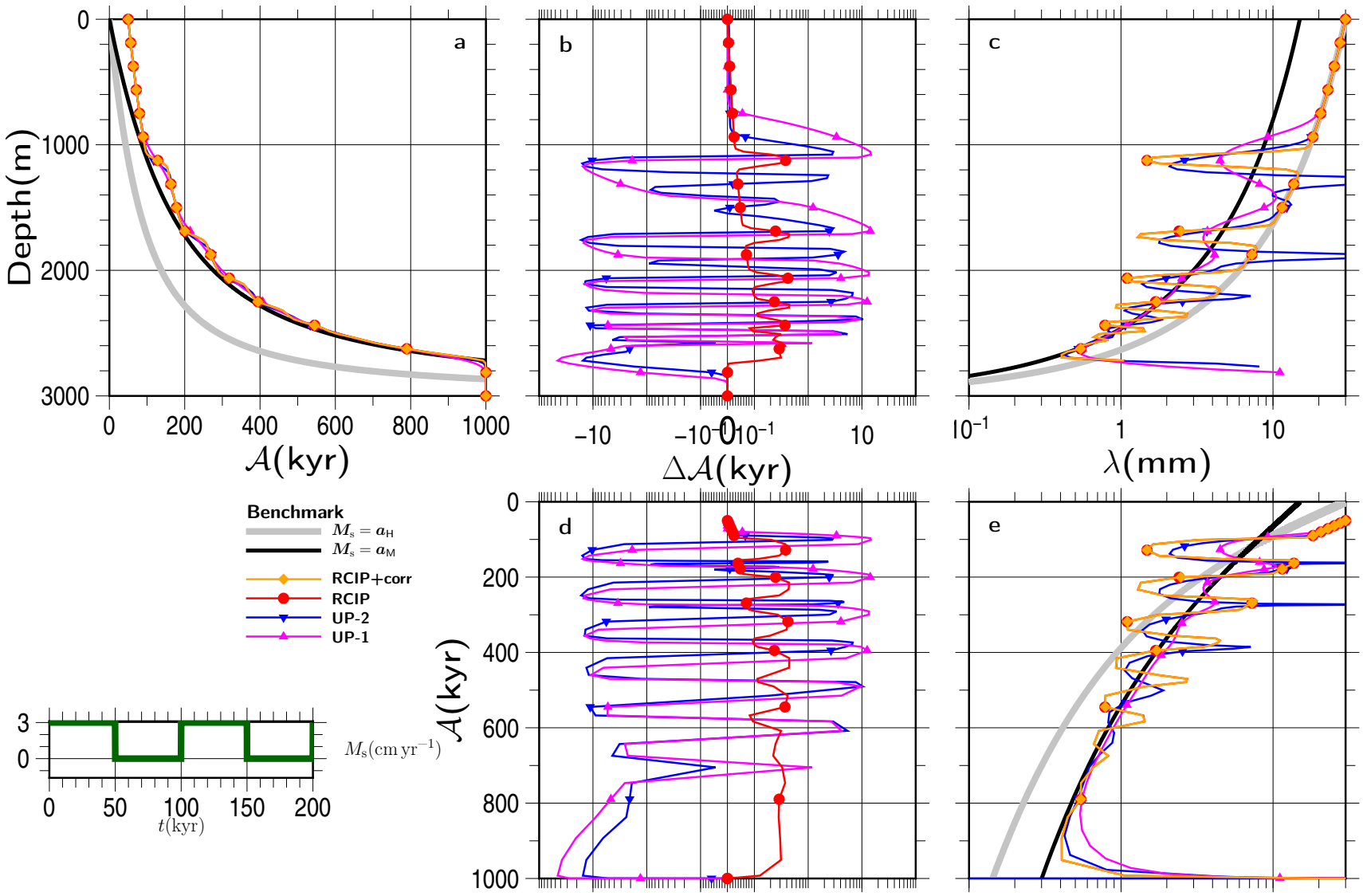

Figure S13
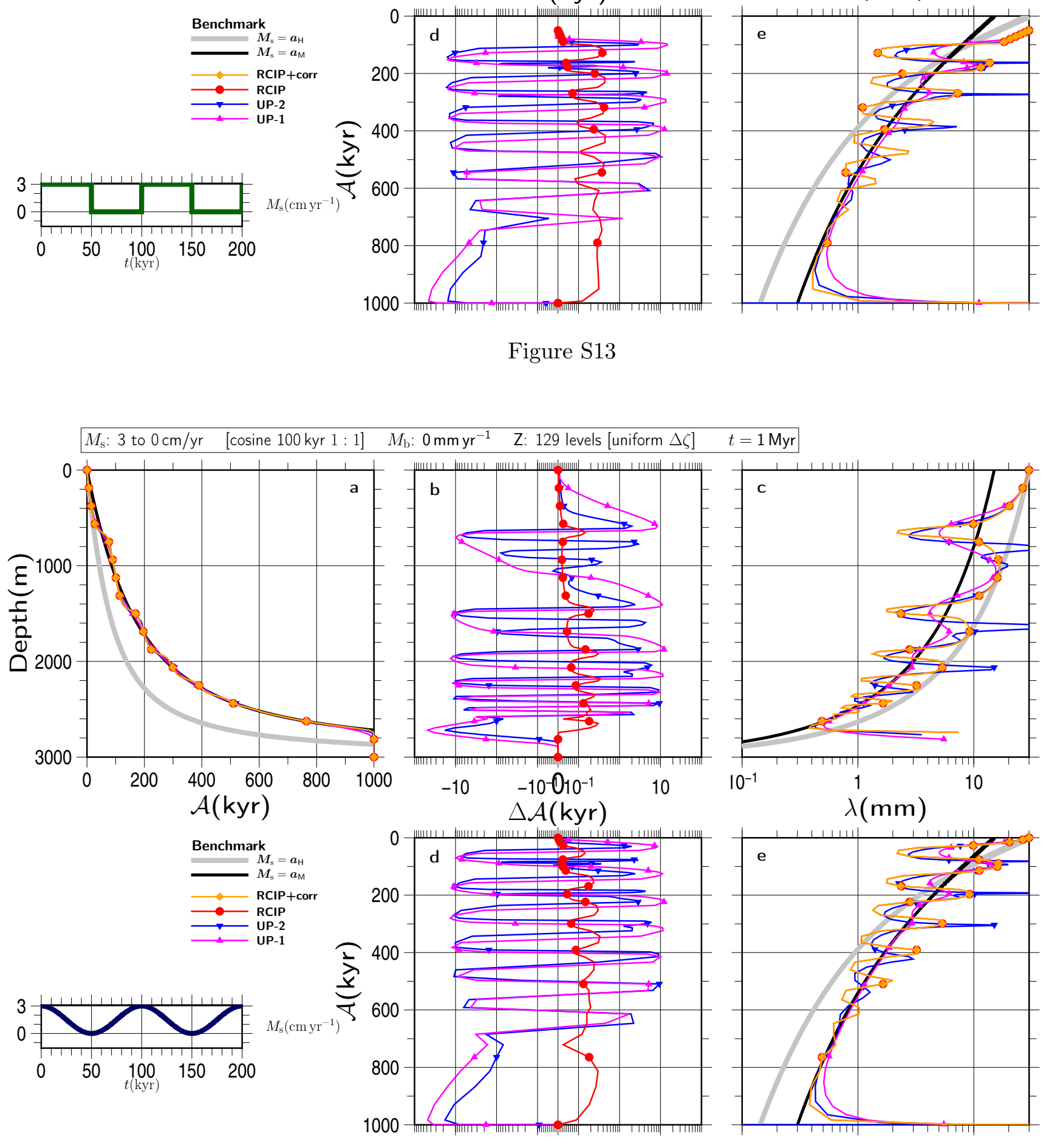

Figure S14 

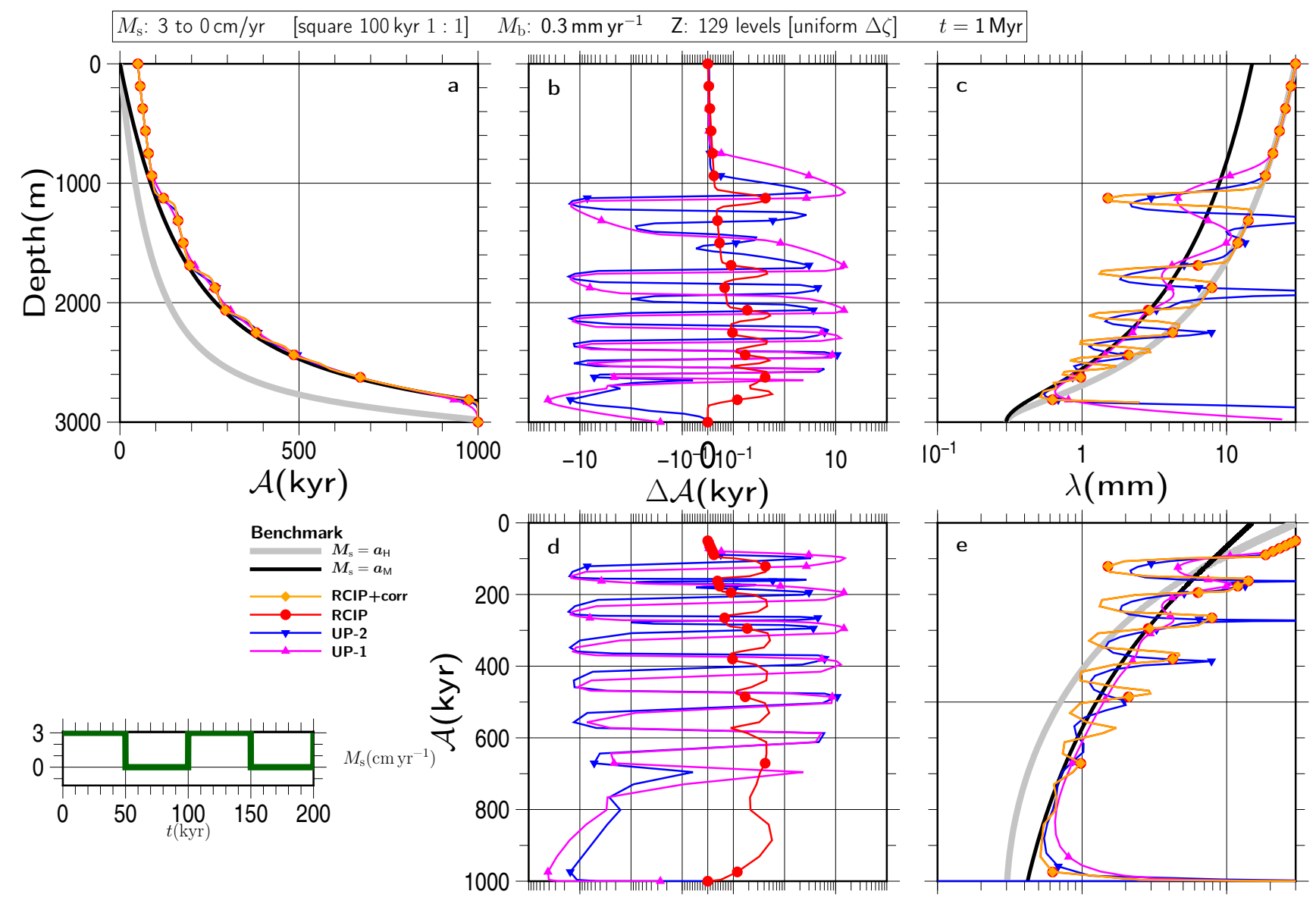

Figure S15
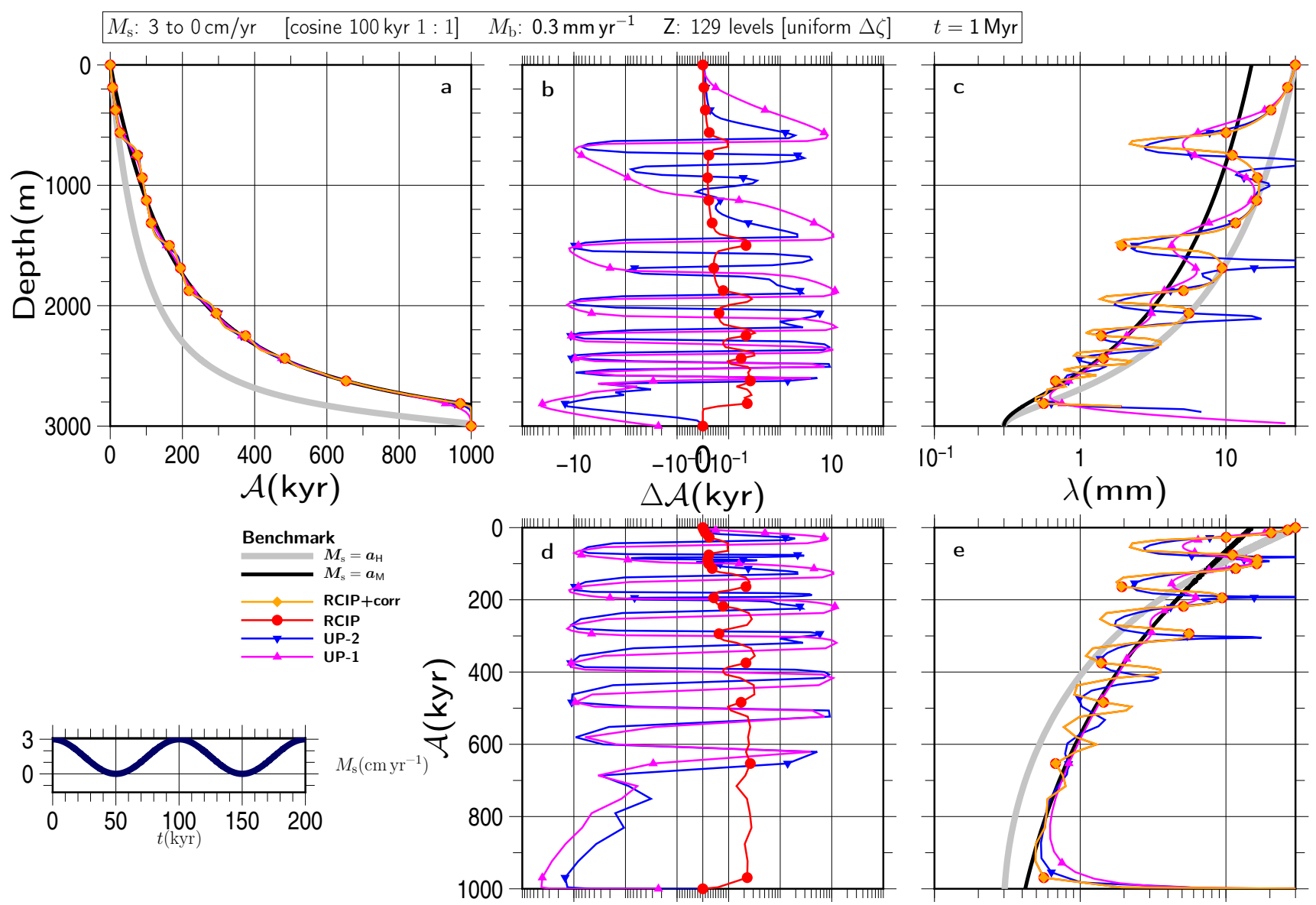

Figure S16 

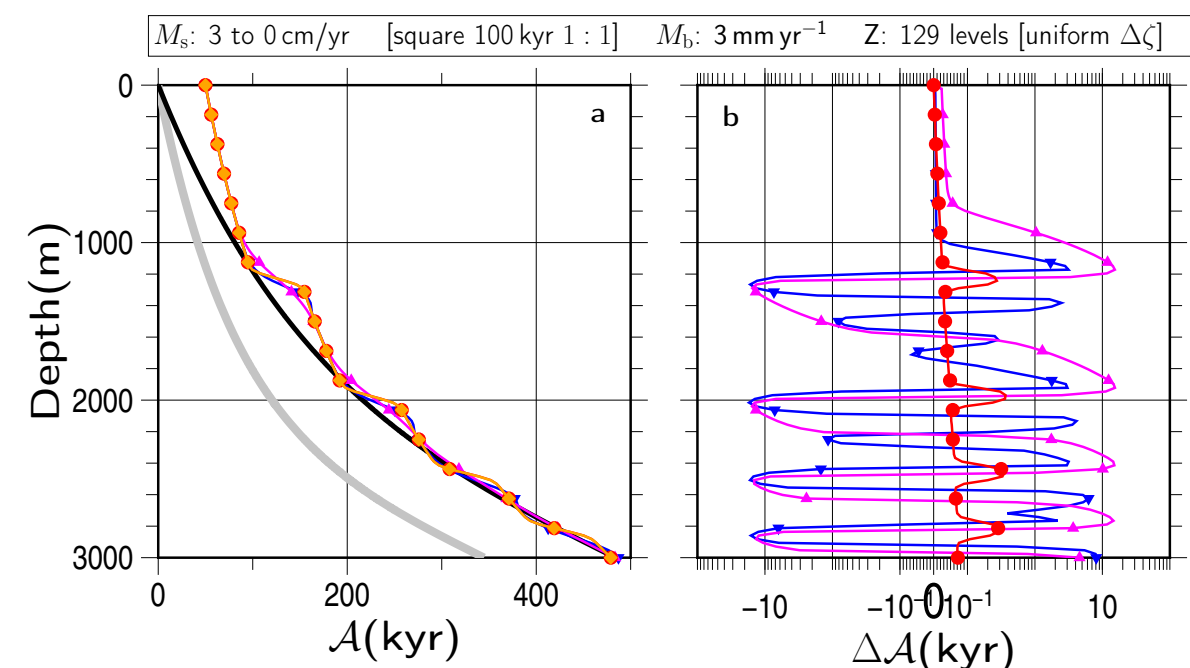

$t=1 \mathrm{Myr}$
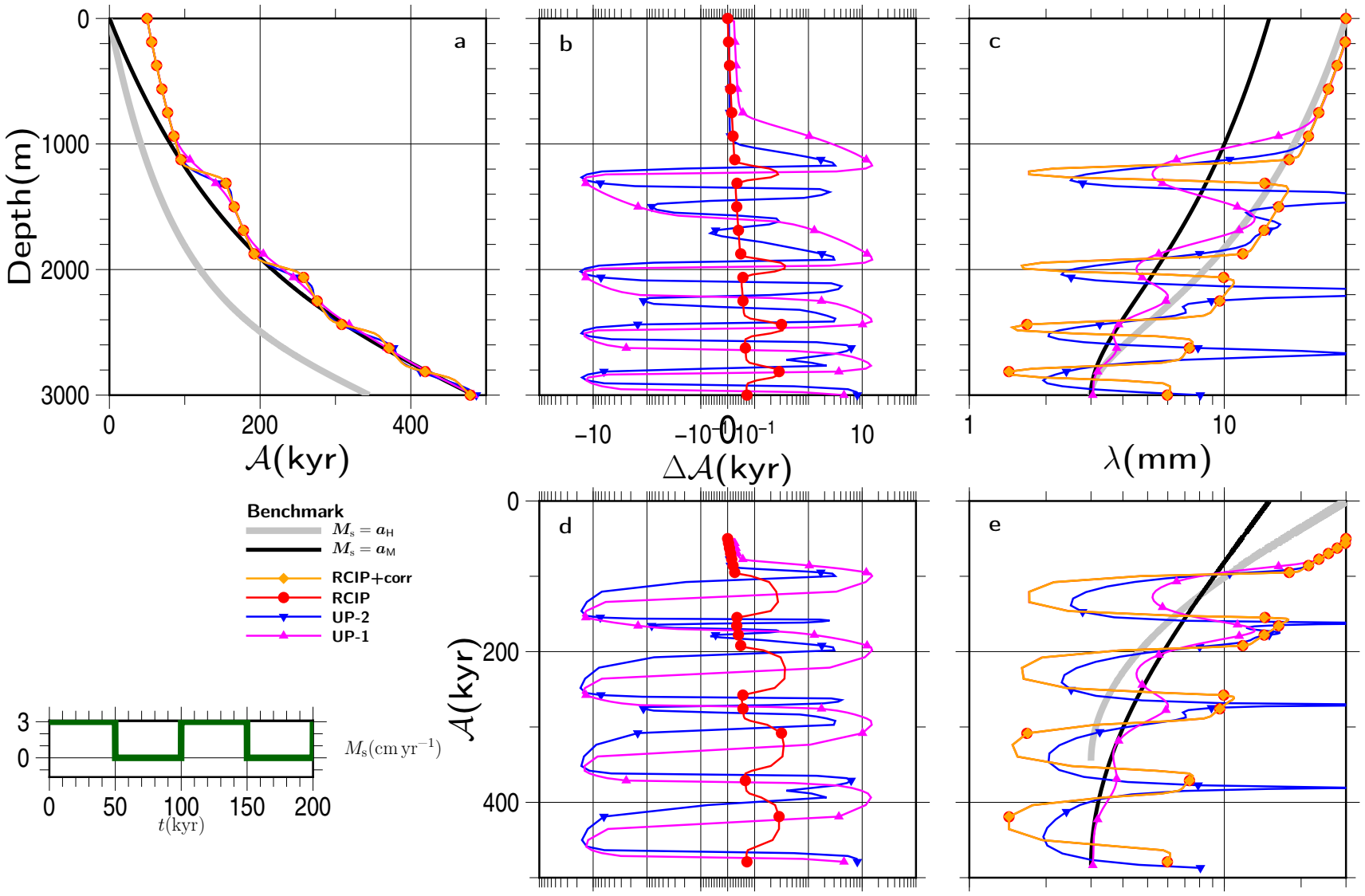

Figure S17
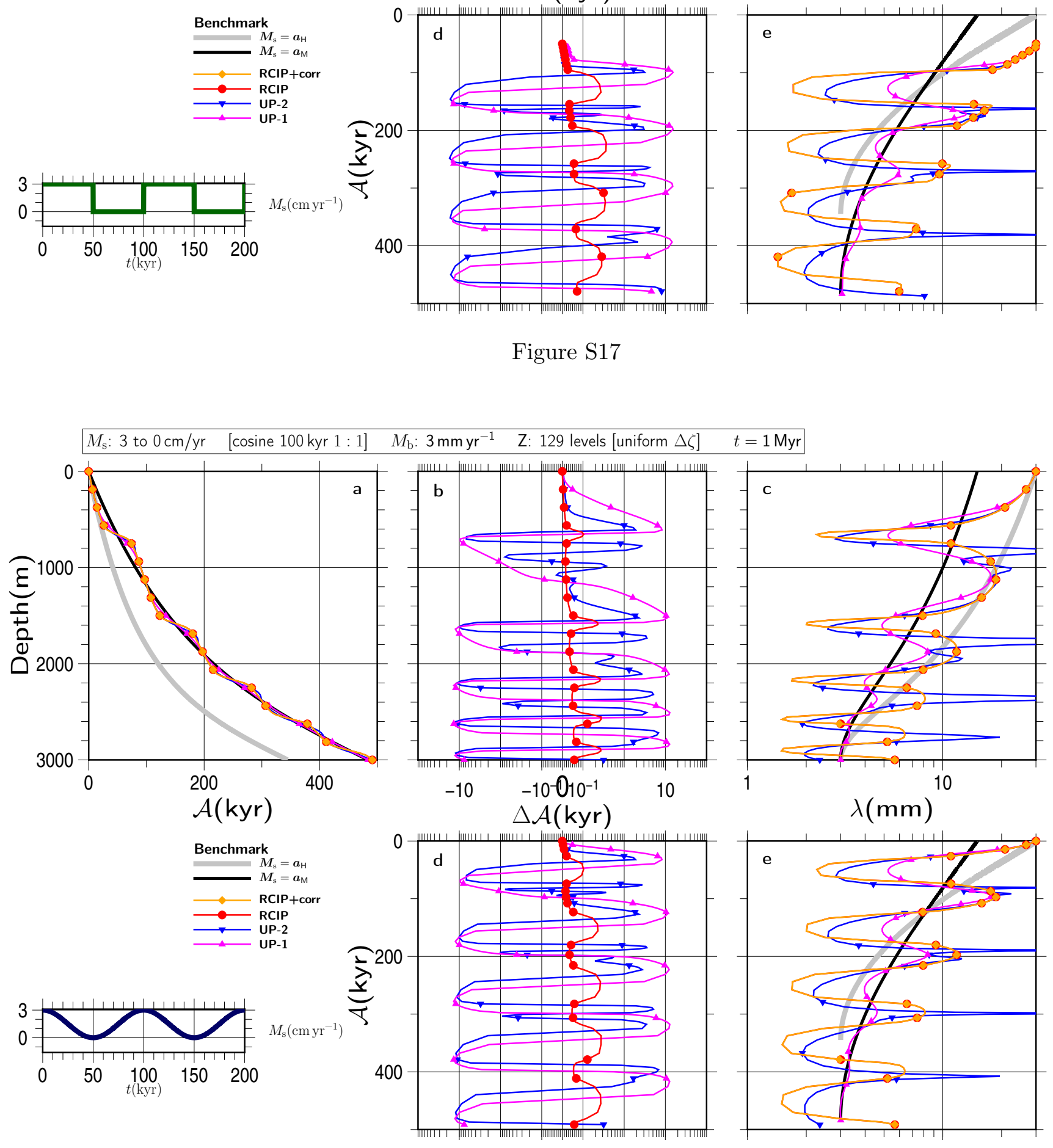

Figure S18 

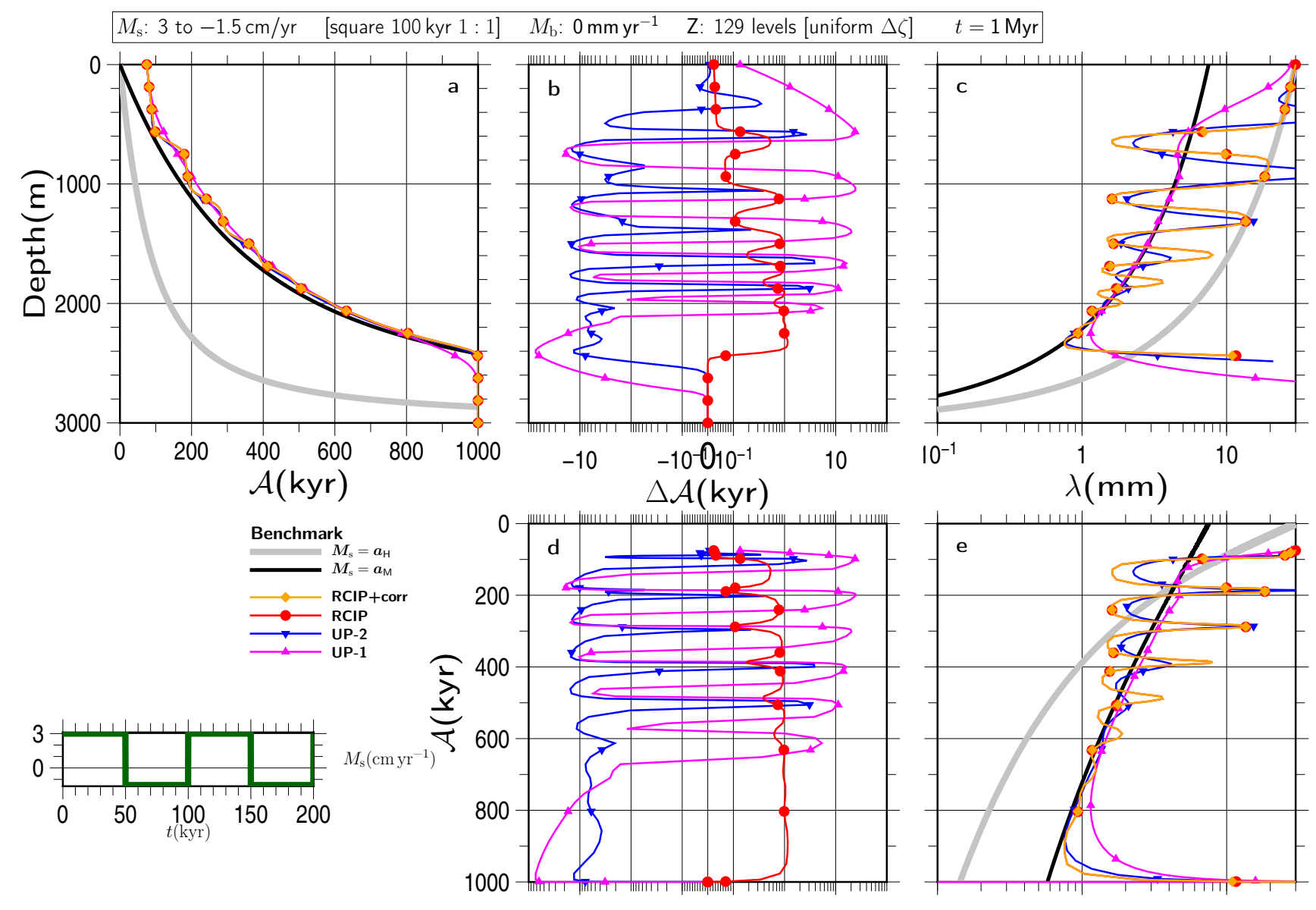

Figure S19
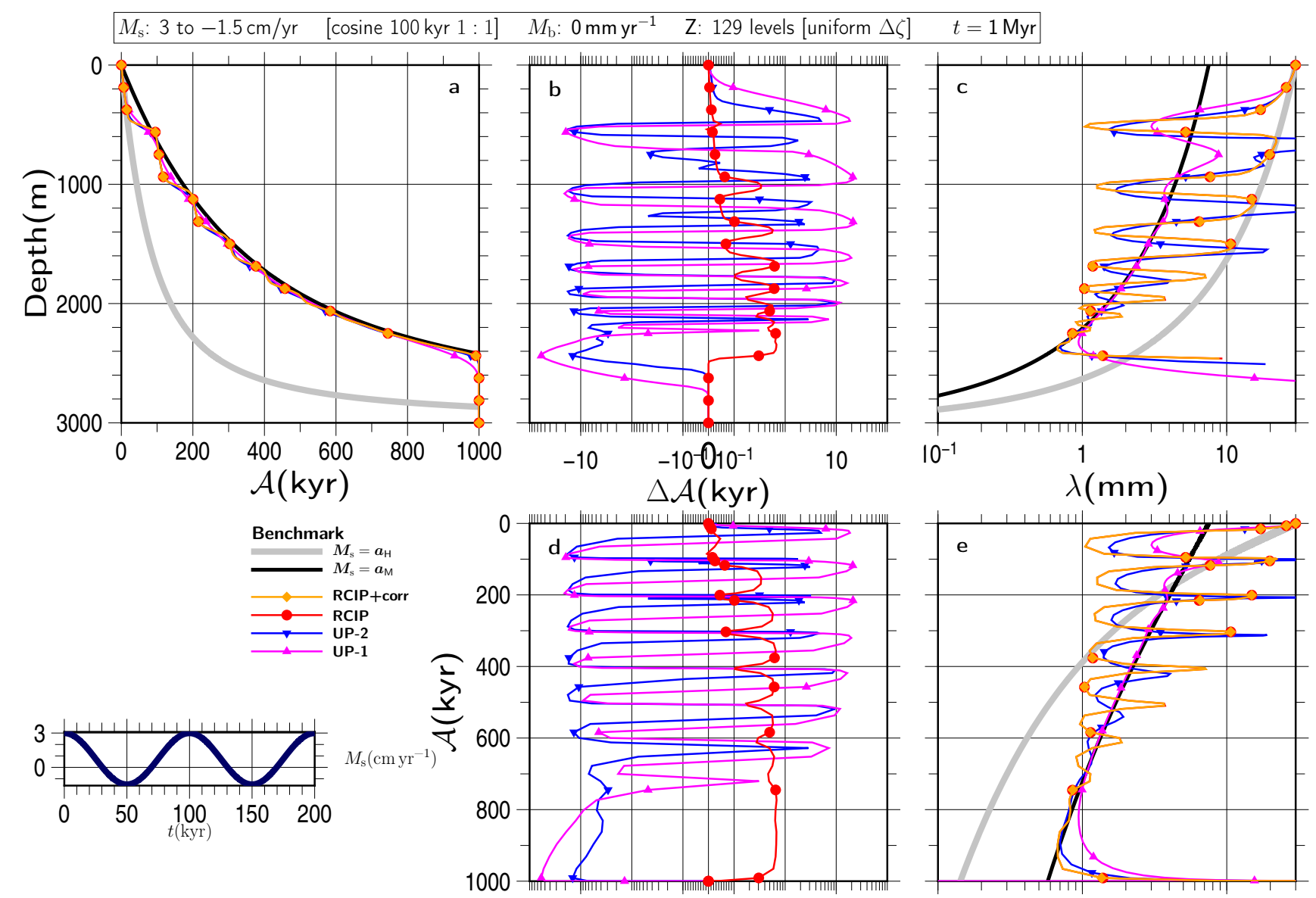

Figure S20 

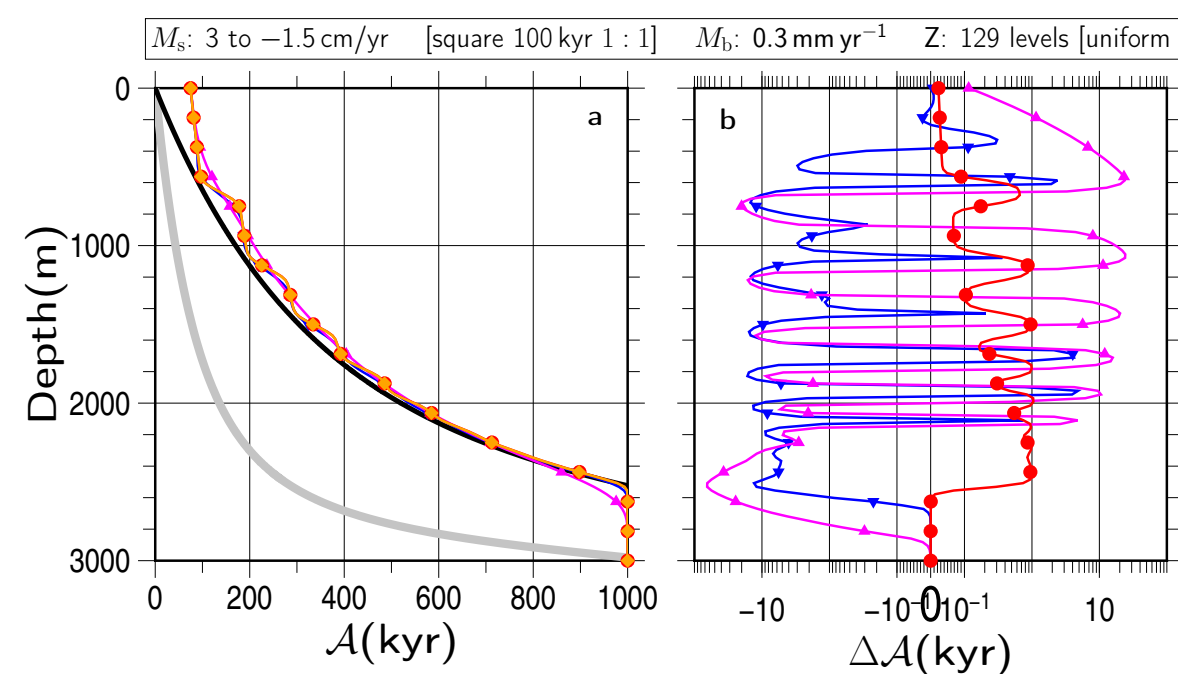

\section{$\Delta \zeta] \quad t=1 \mathrm{Myr}$}
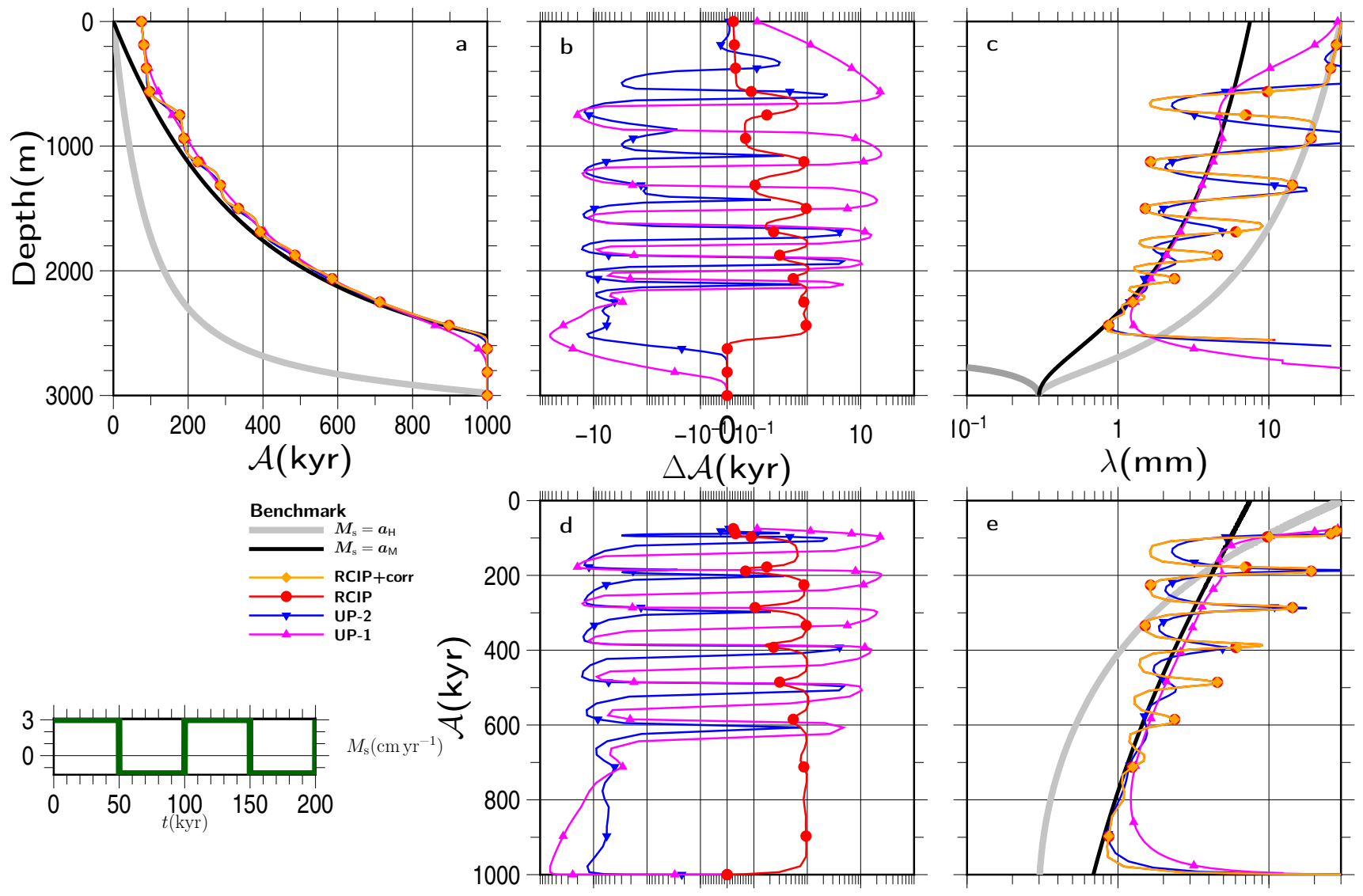

Figure S21
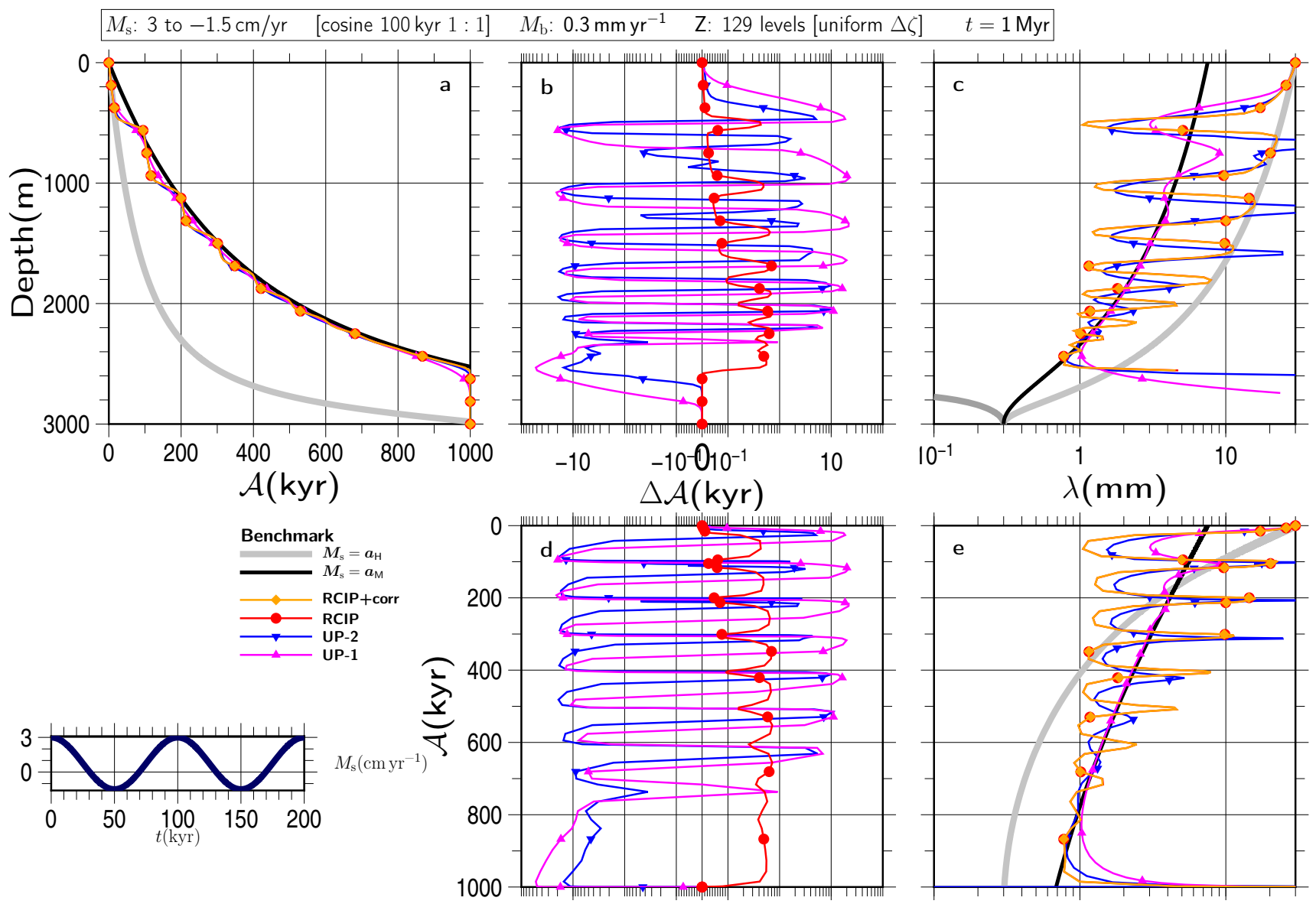

Figure S22 

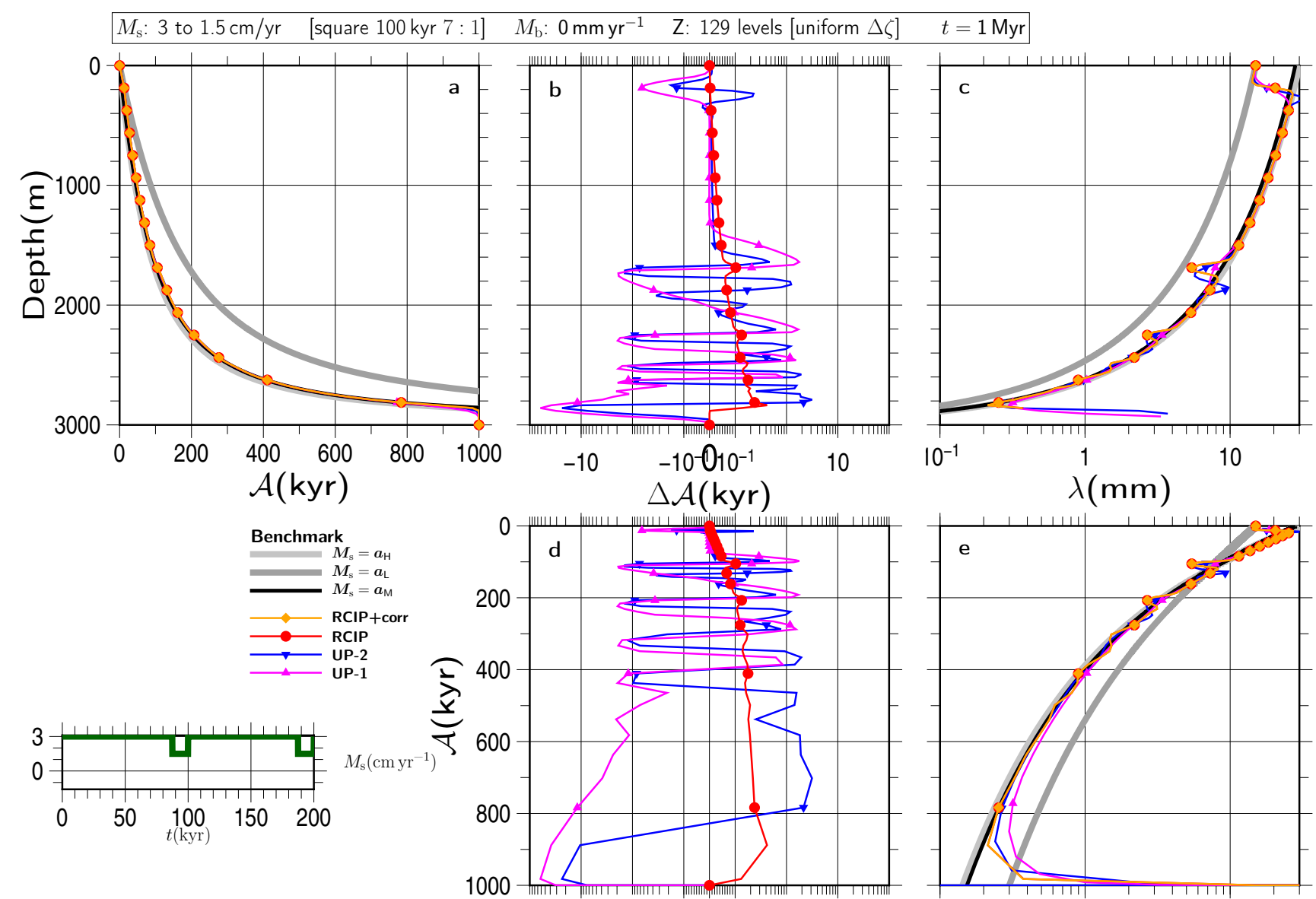

Figure S23
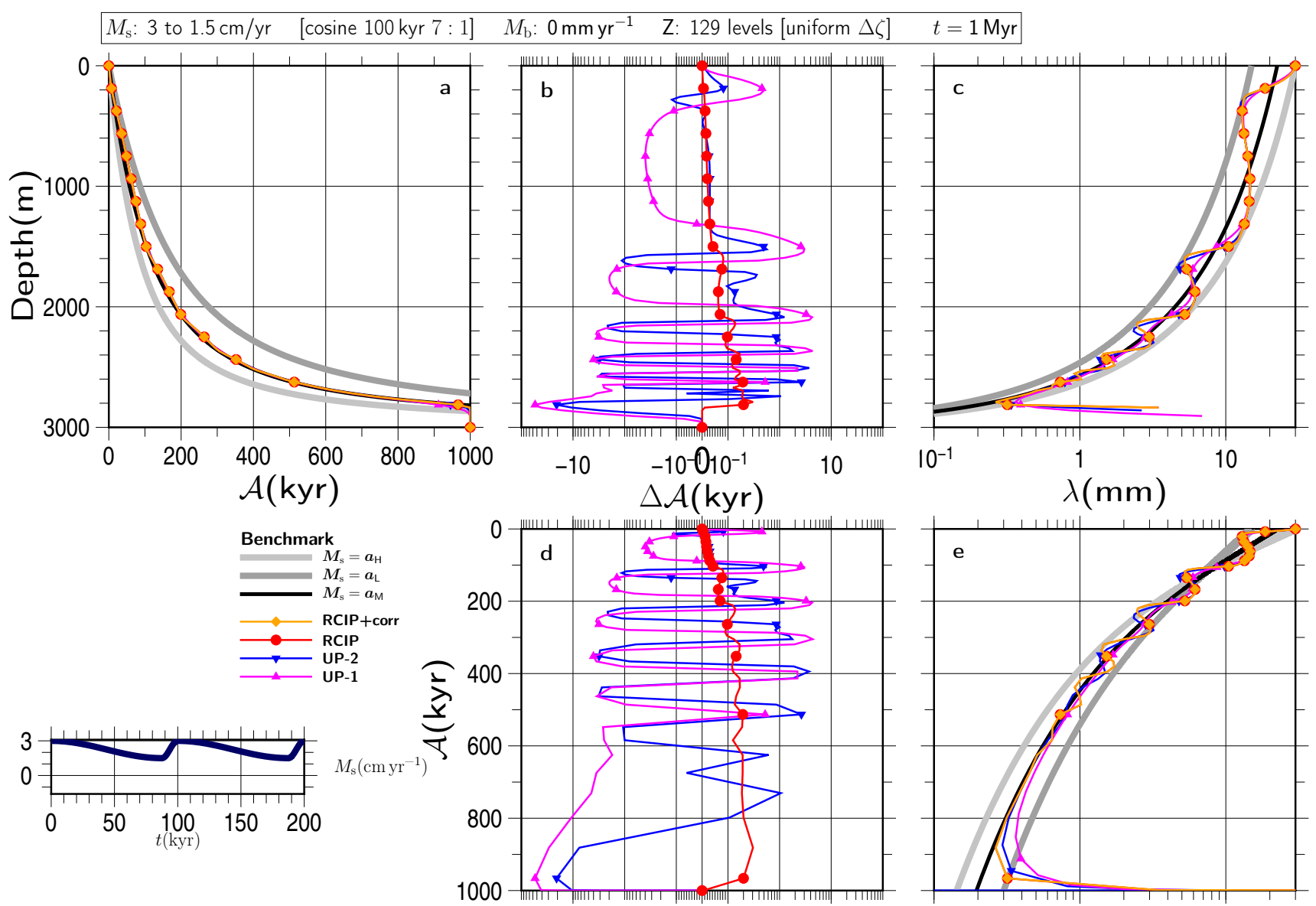

Figure S24 

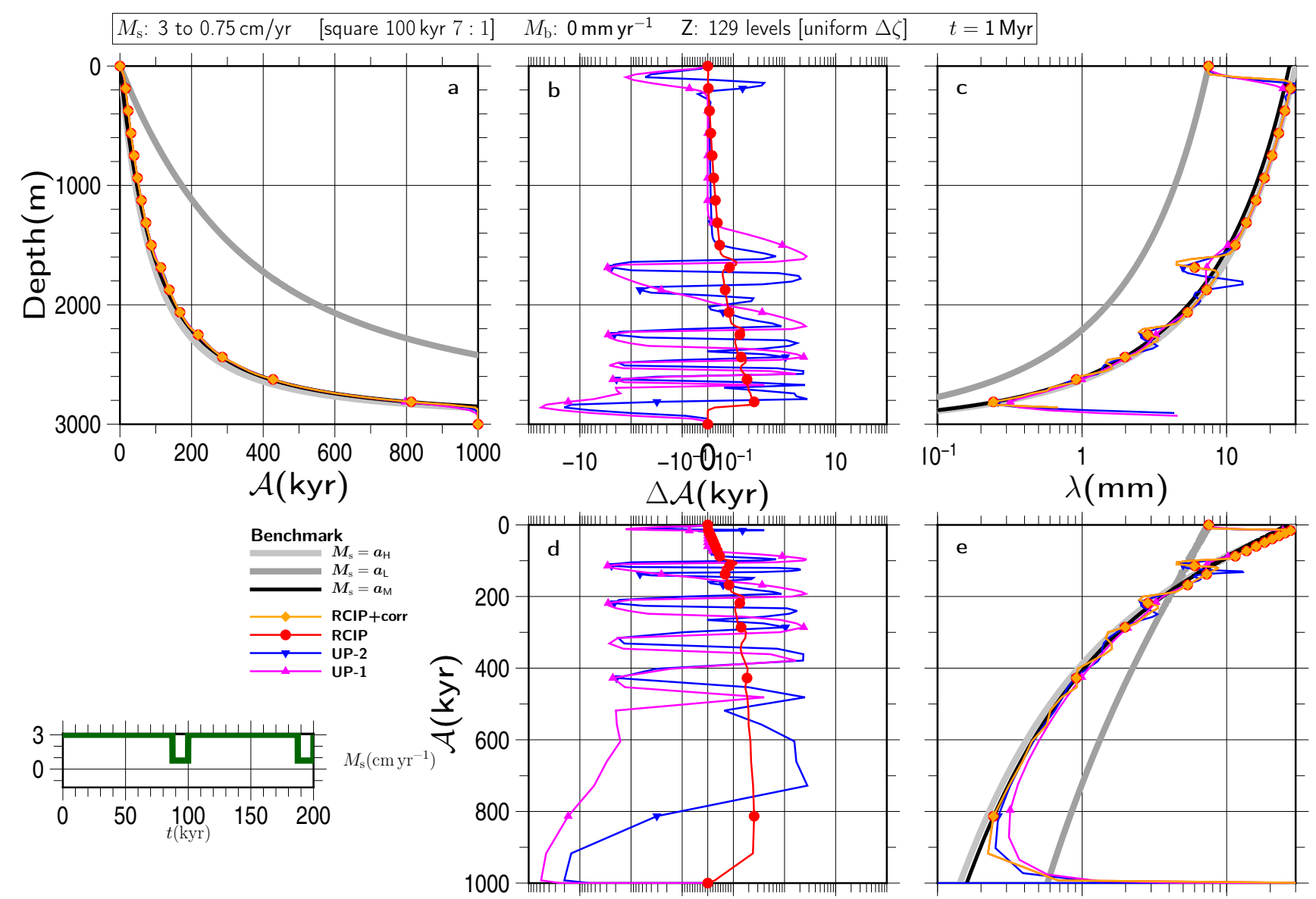

Figure S25
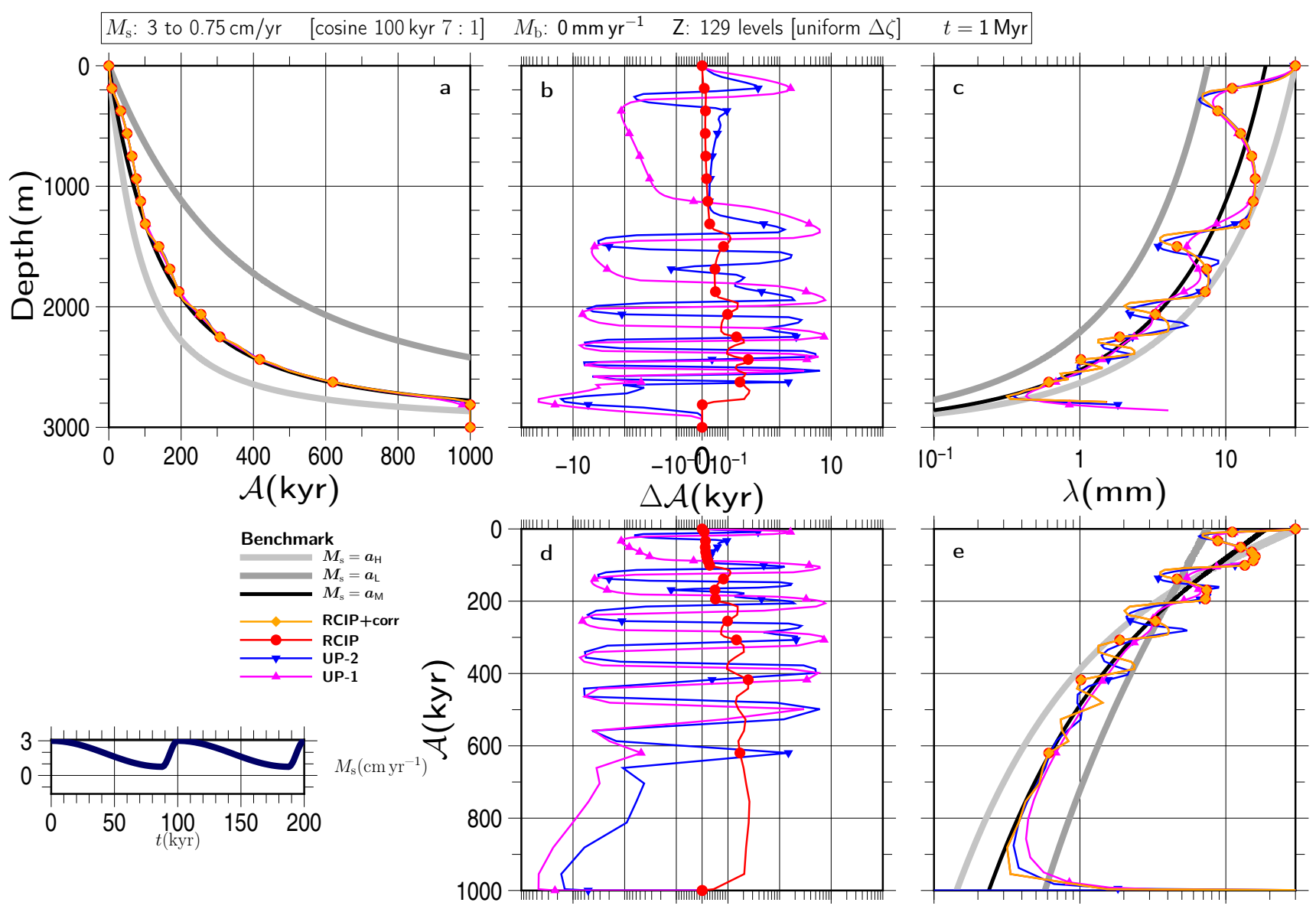

Figure S26 

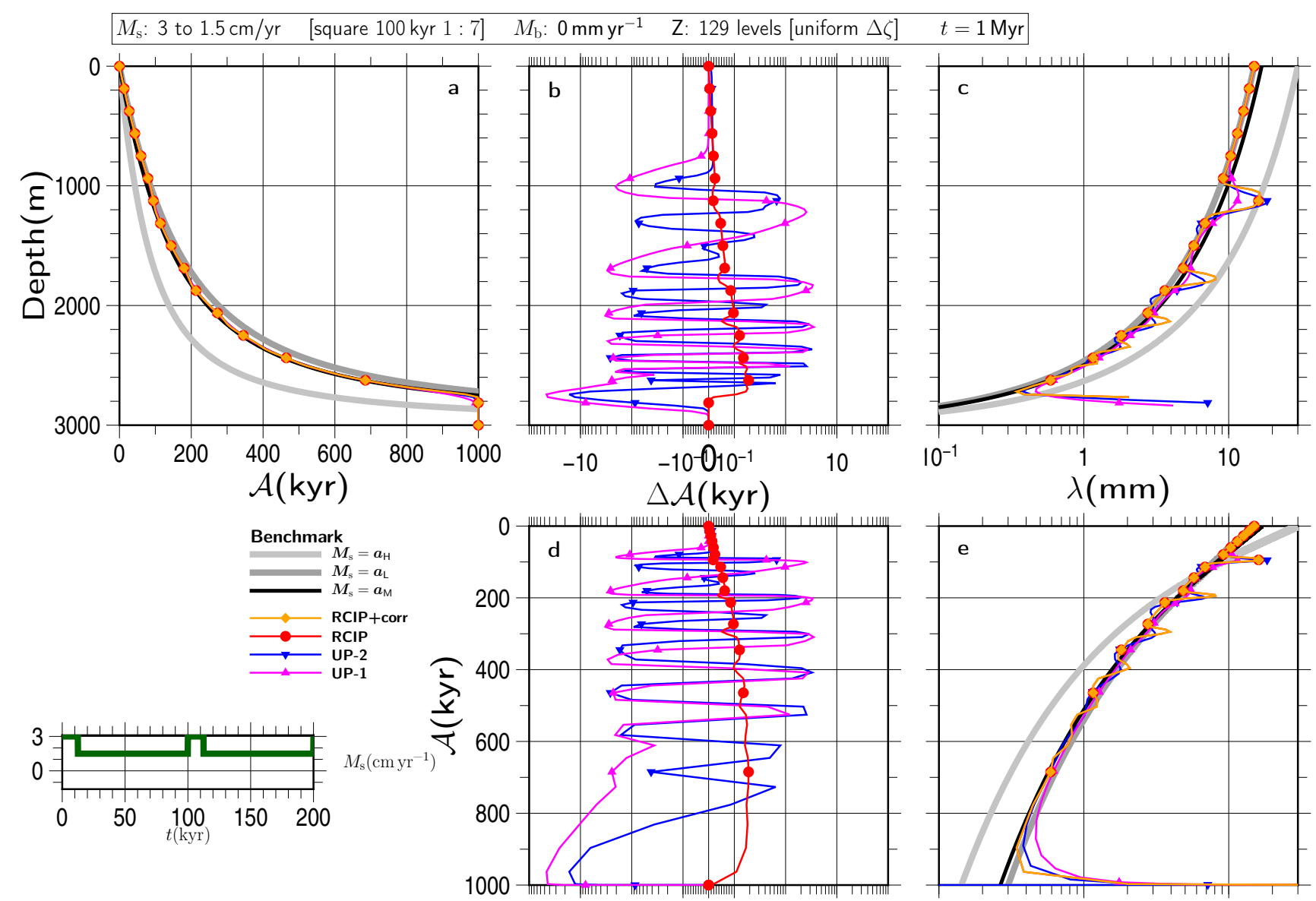

Figure S27
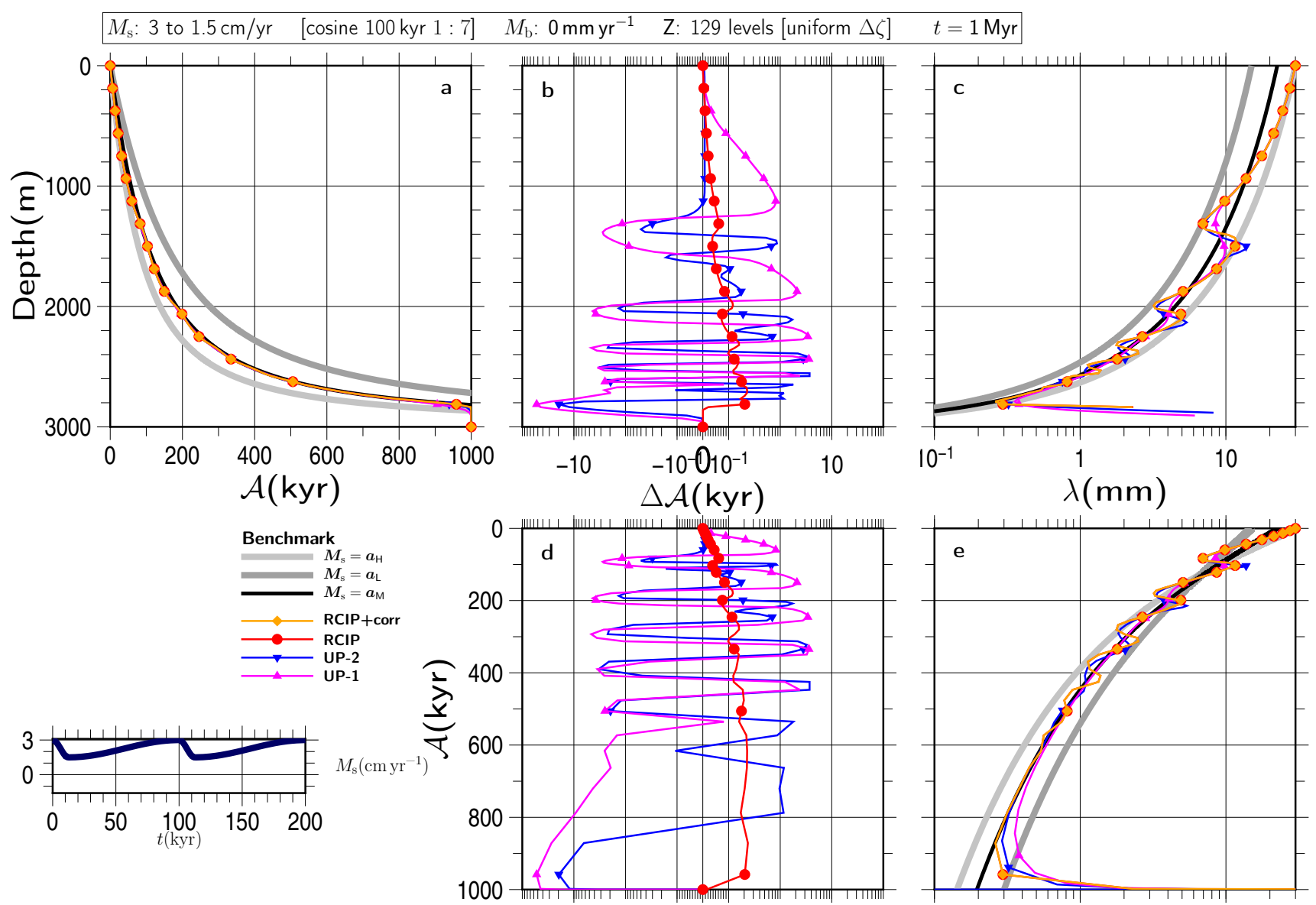

Figure S28 

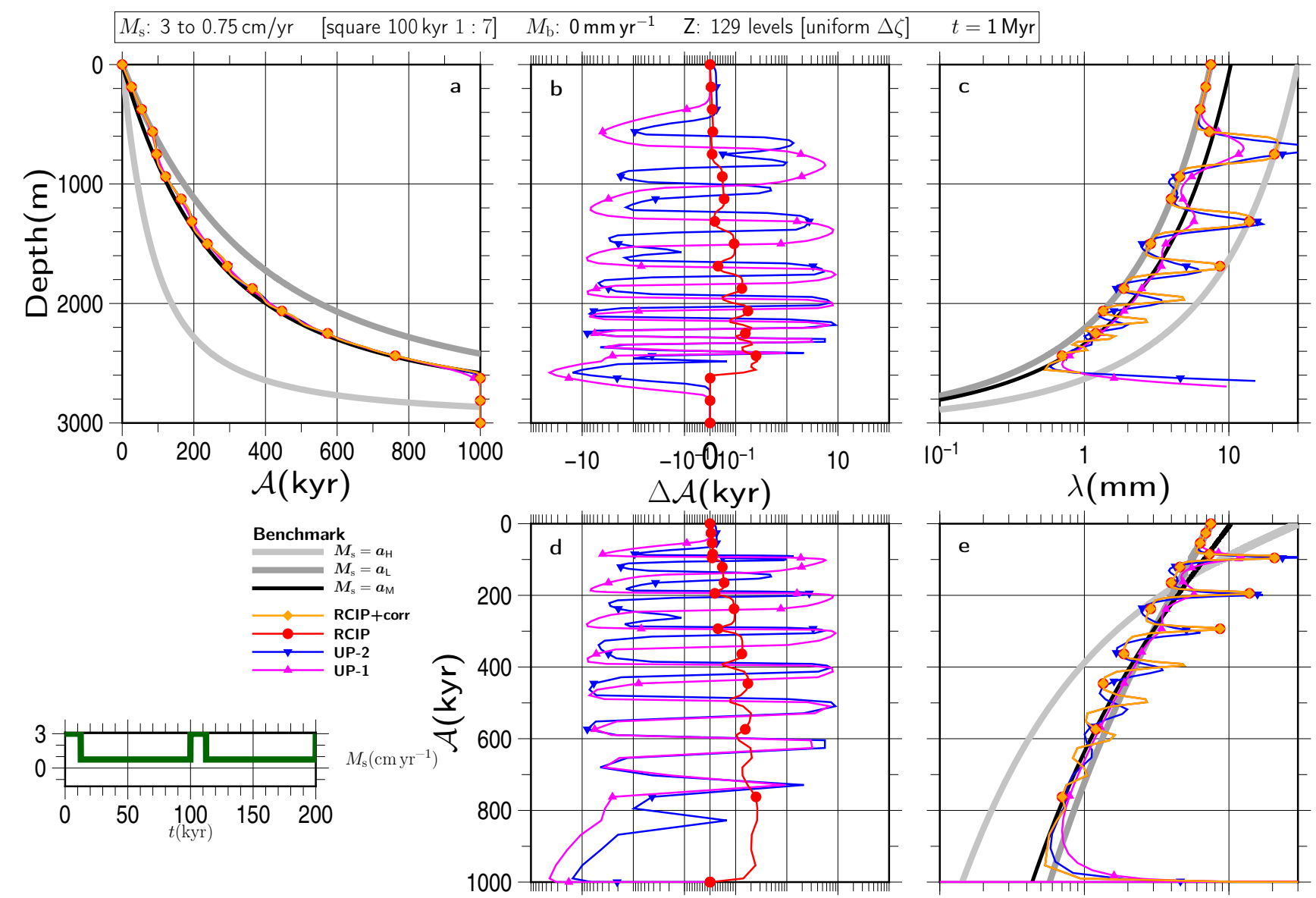

Figure S29
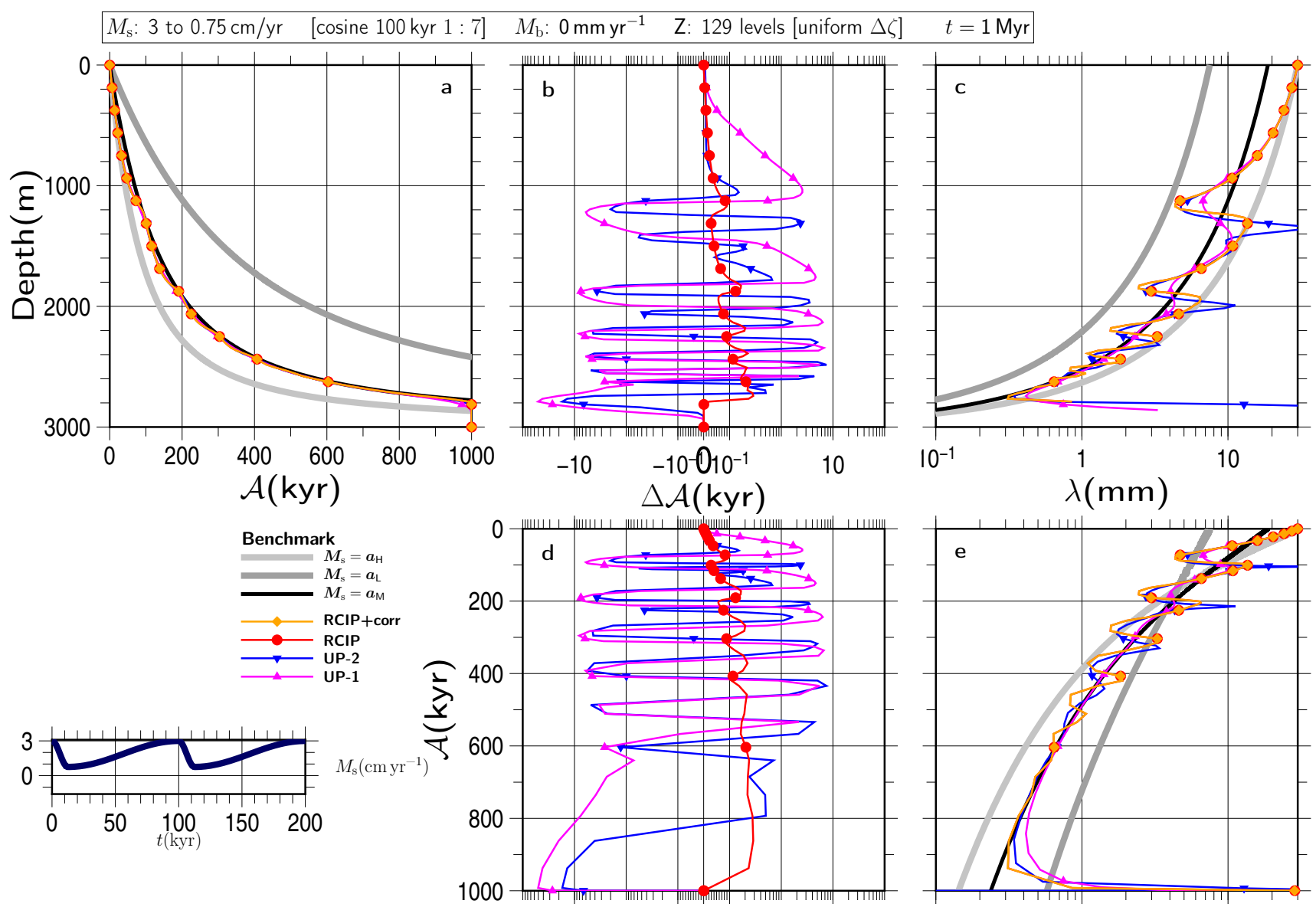

Figure S30 

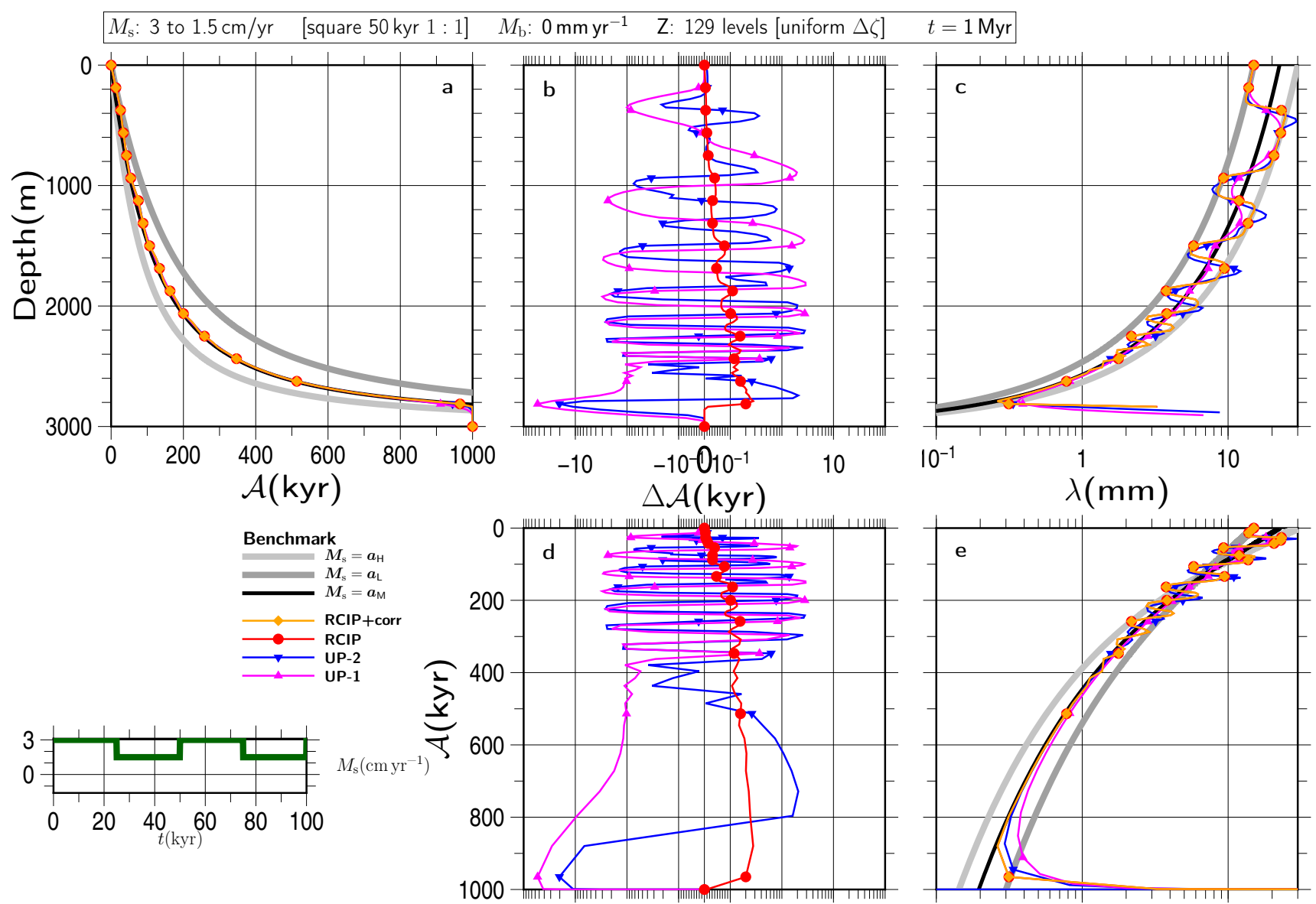

Figure S31
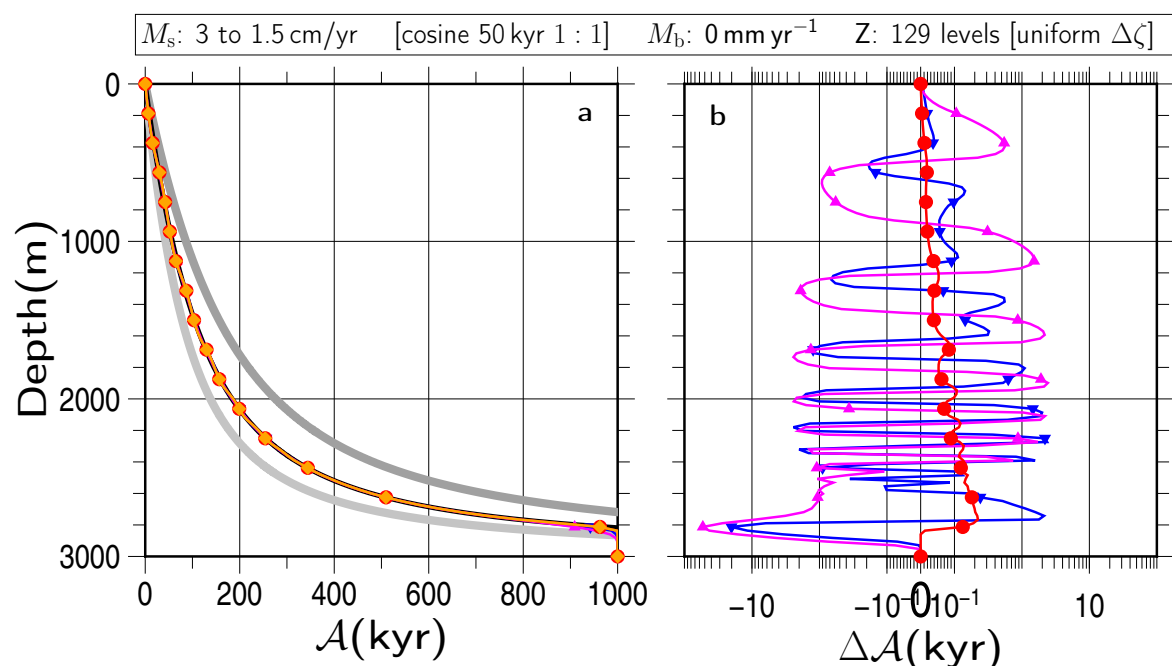

$t=1 \mathrm{Myr}$
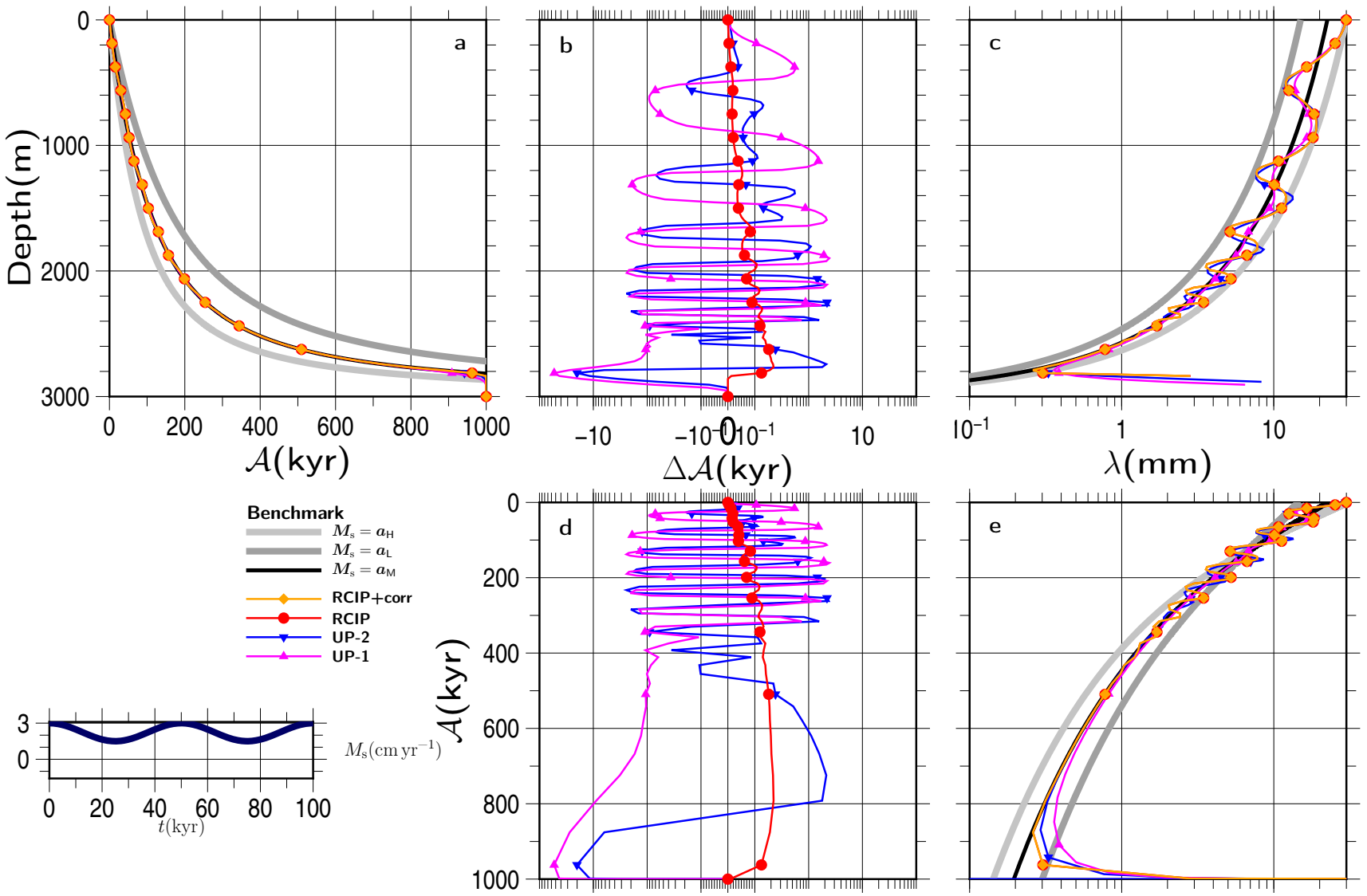

Figure S32 

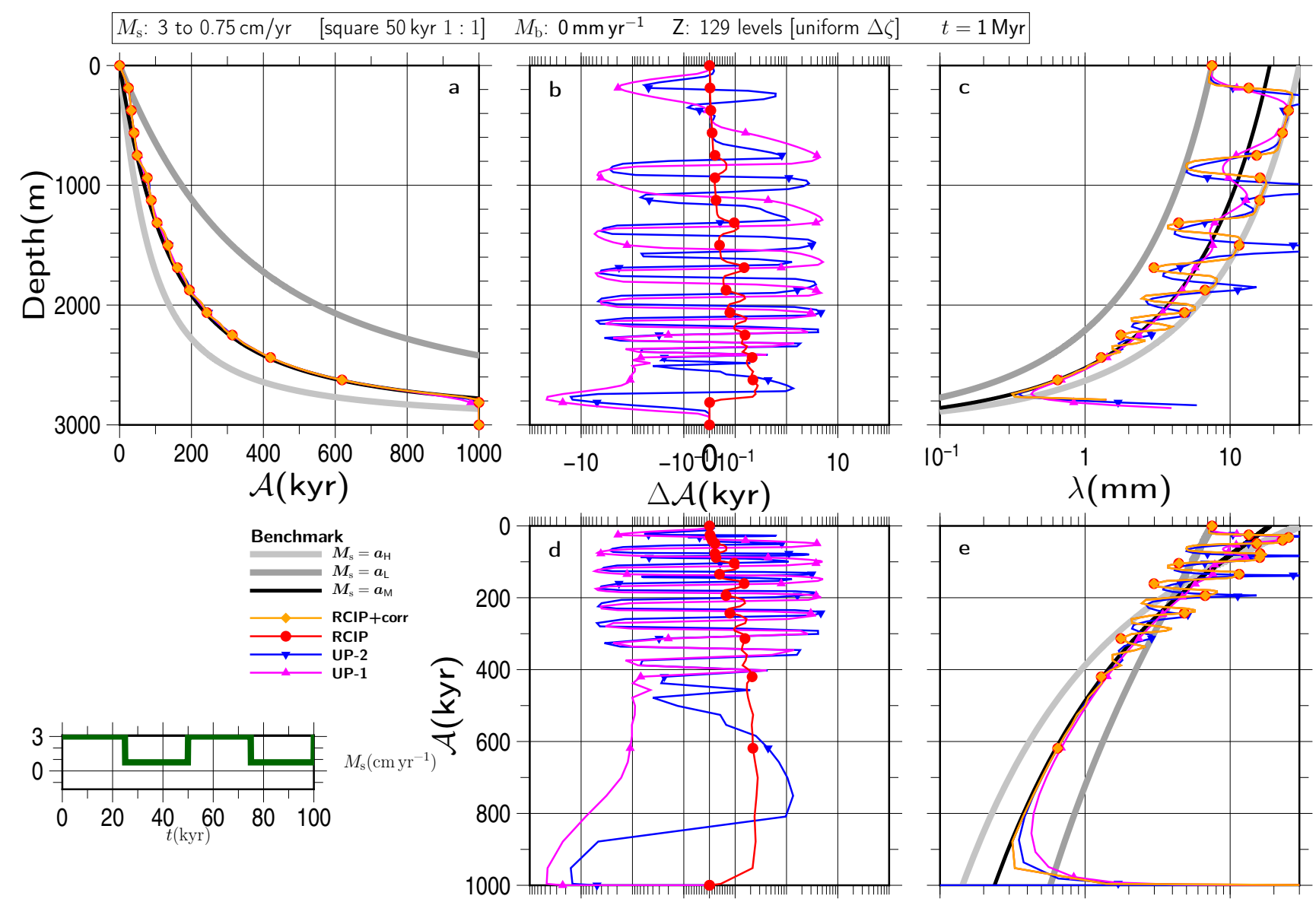

Figure S33
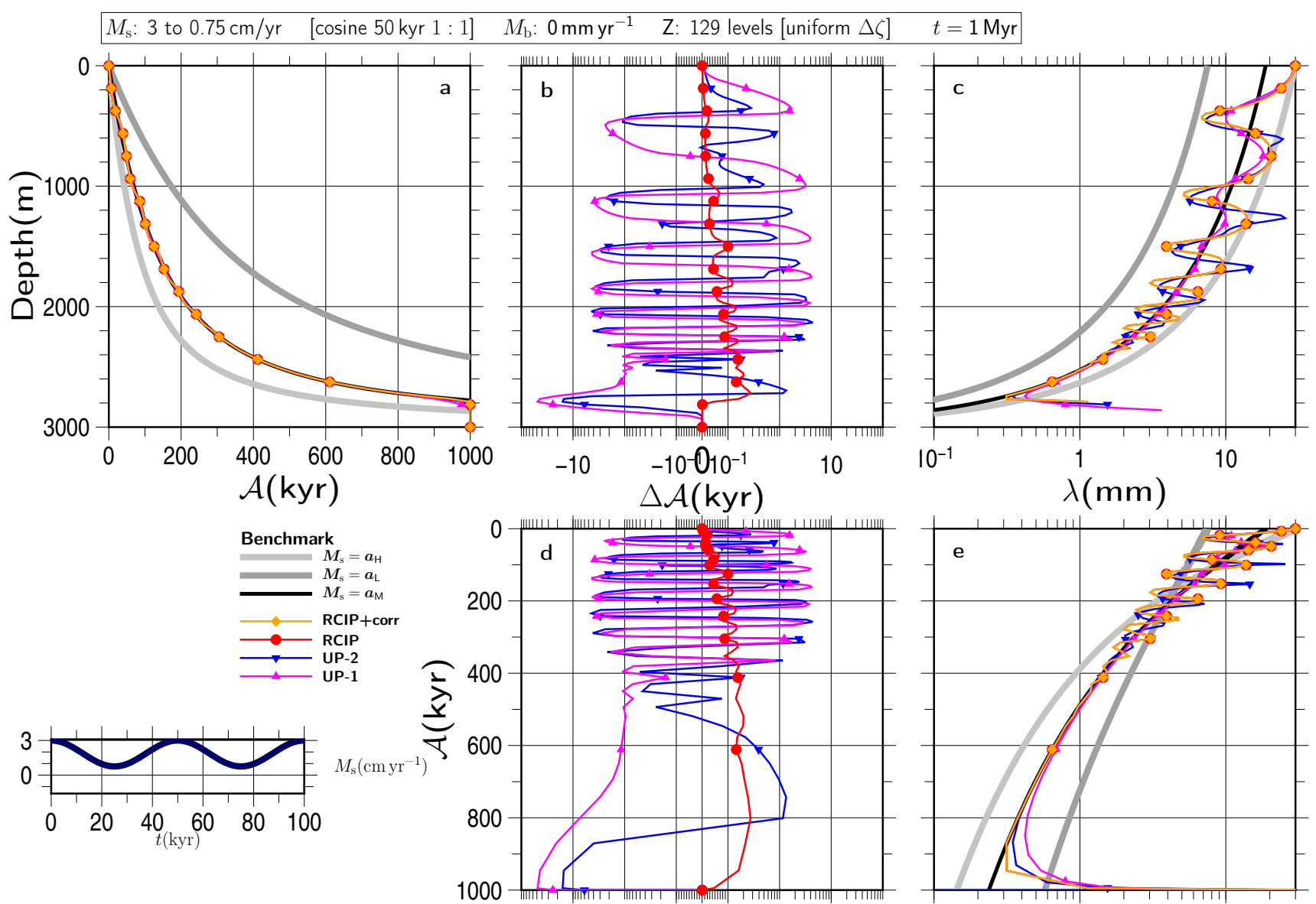

Figure S34 

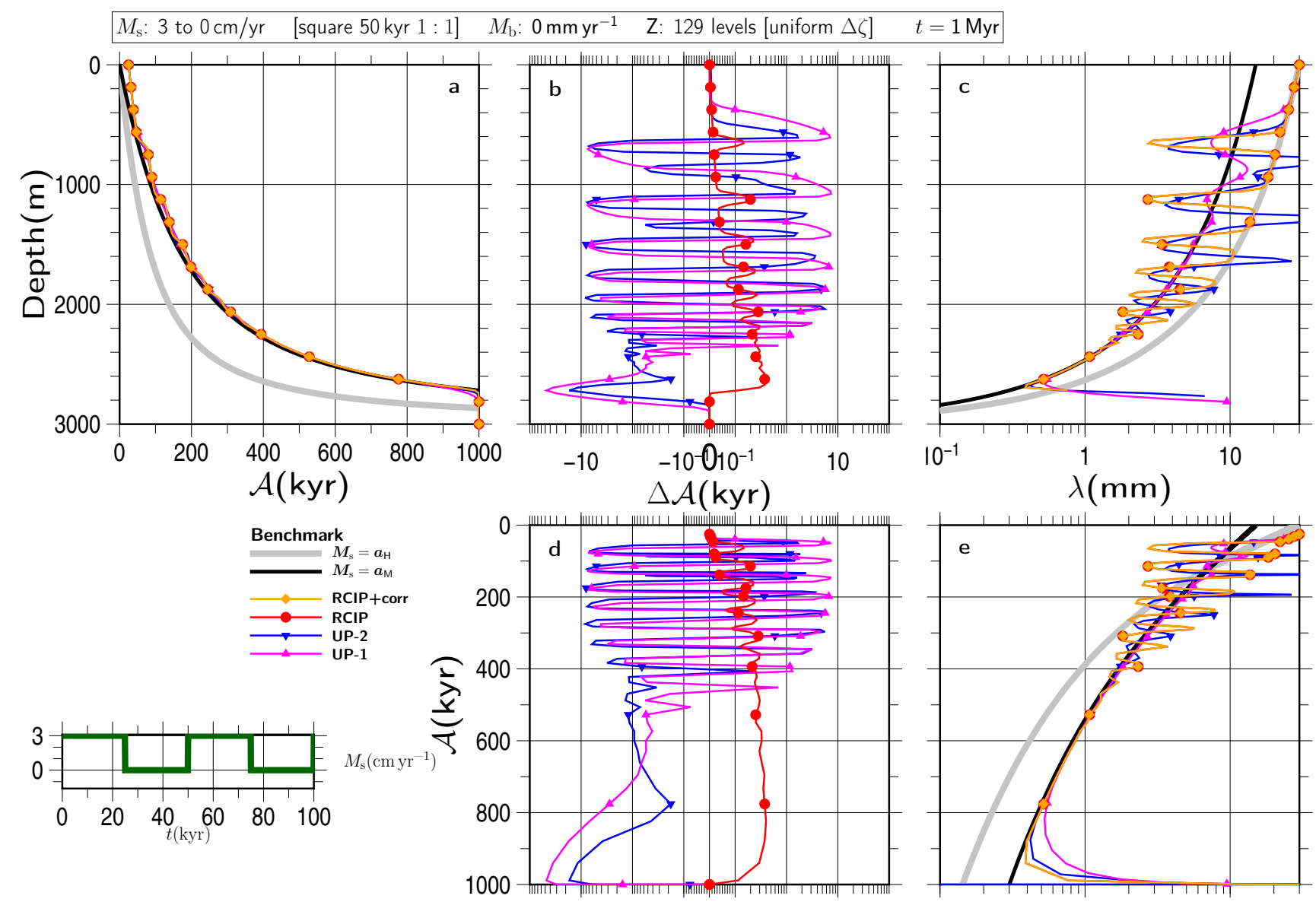

Figure S35
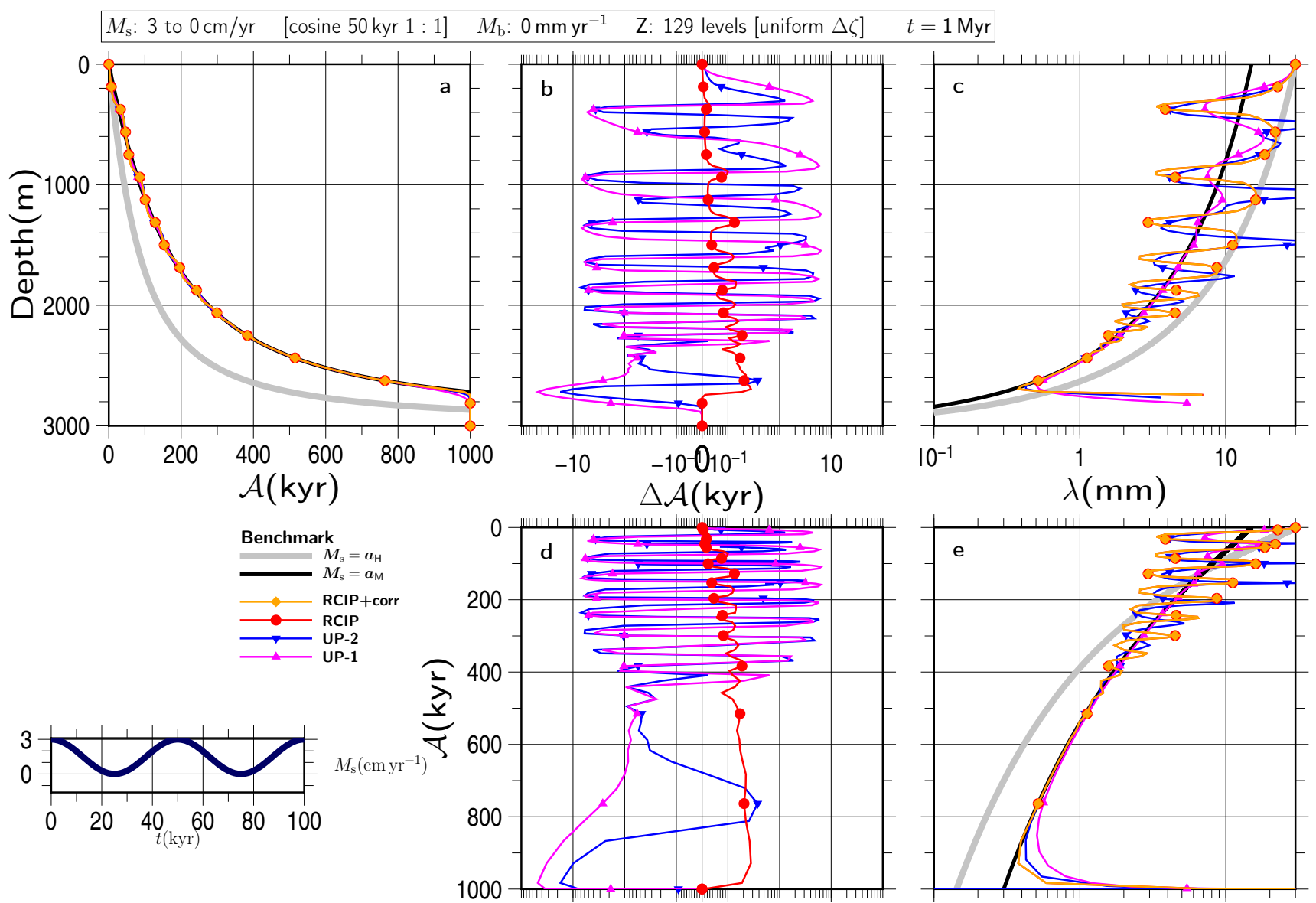

Figure S36 

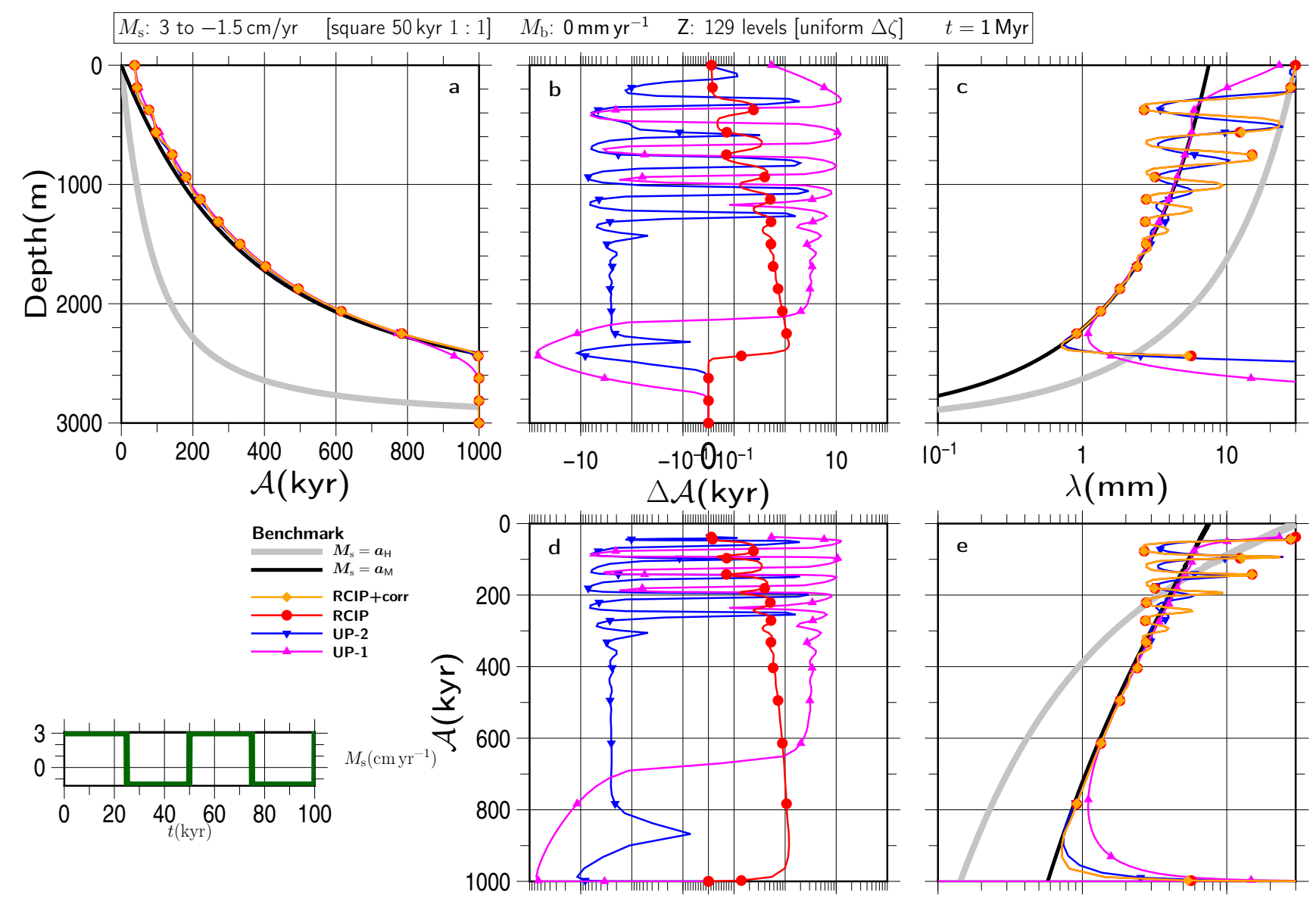

Figure S37
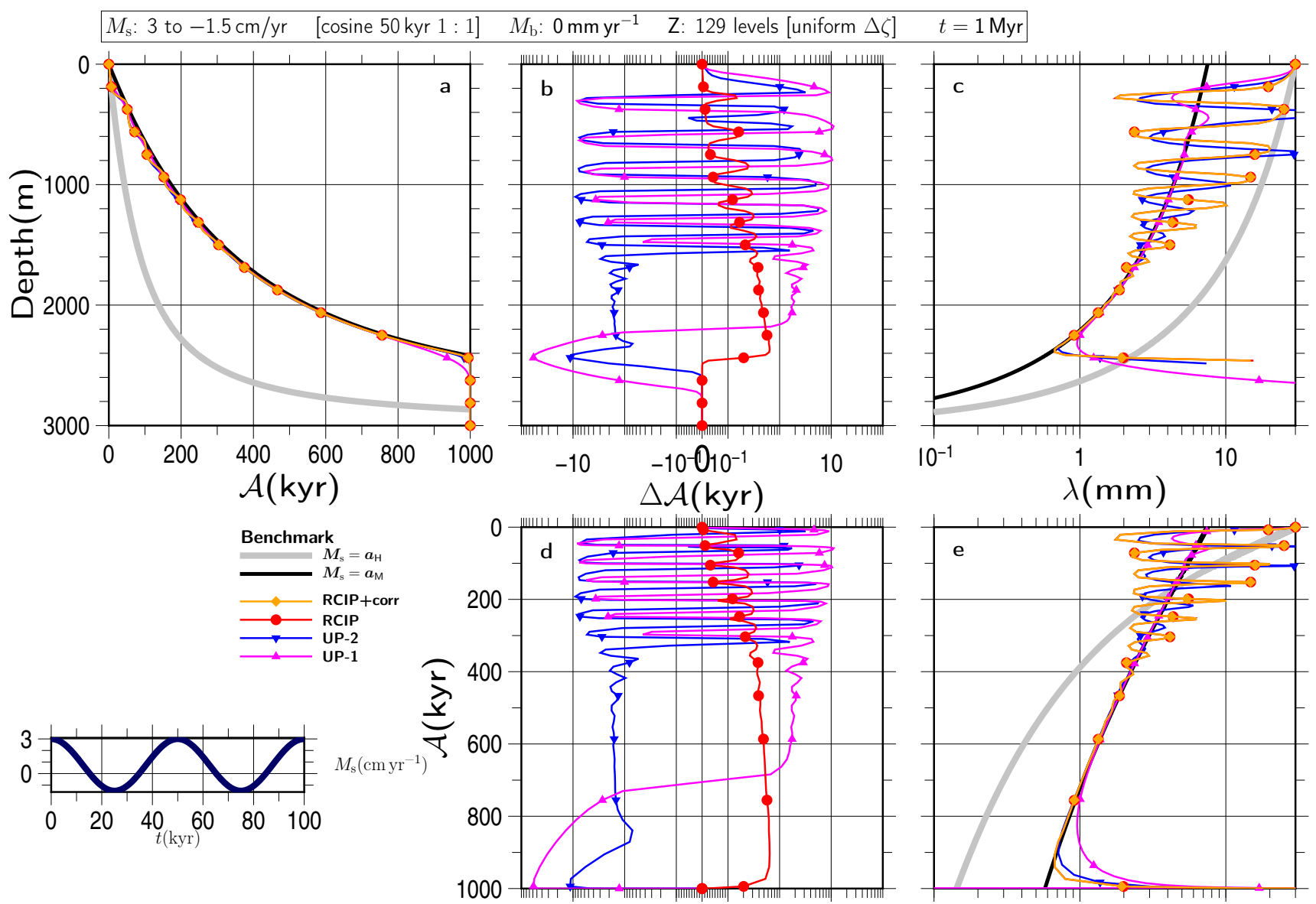

Figure S38 

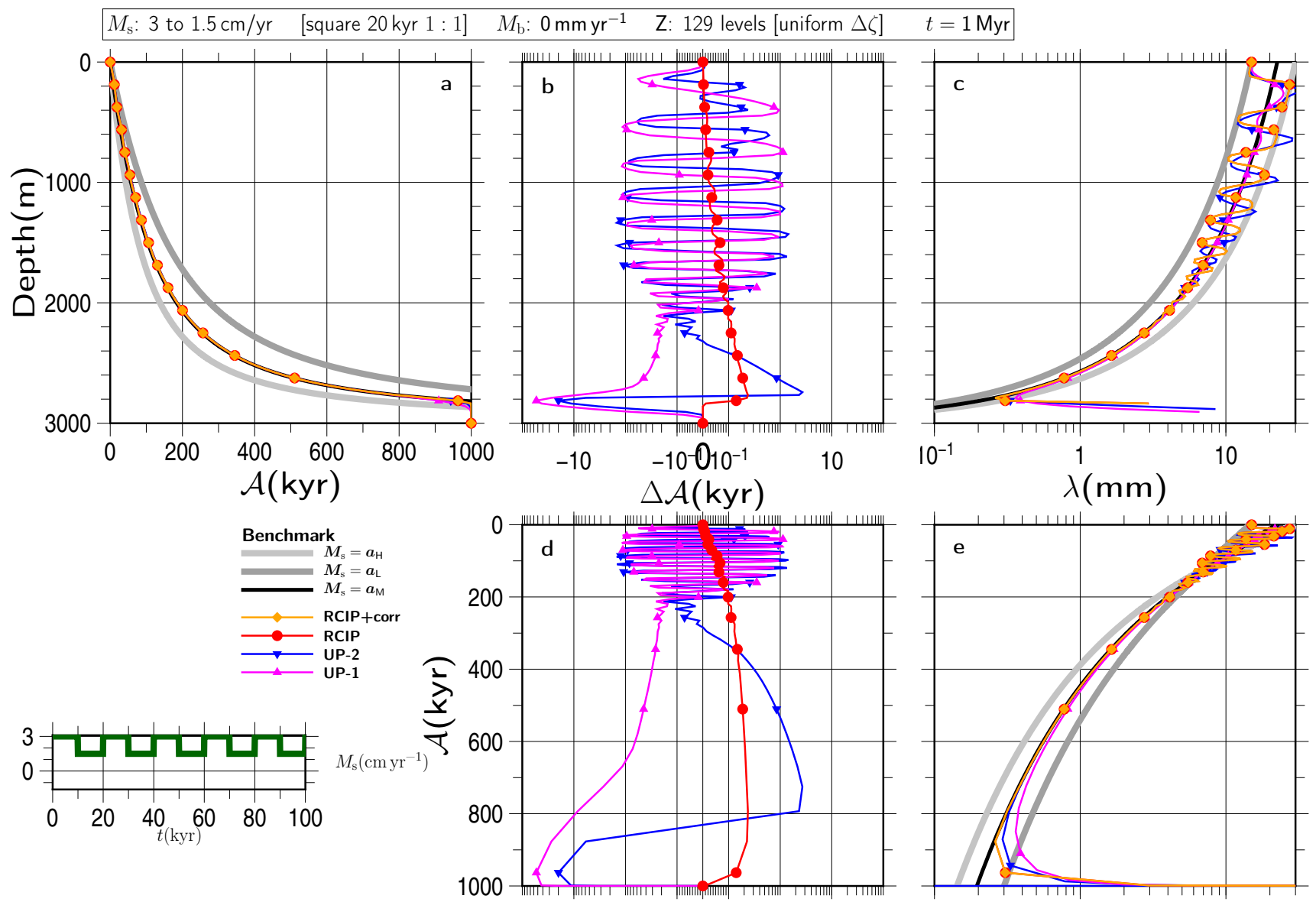

Figure S39
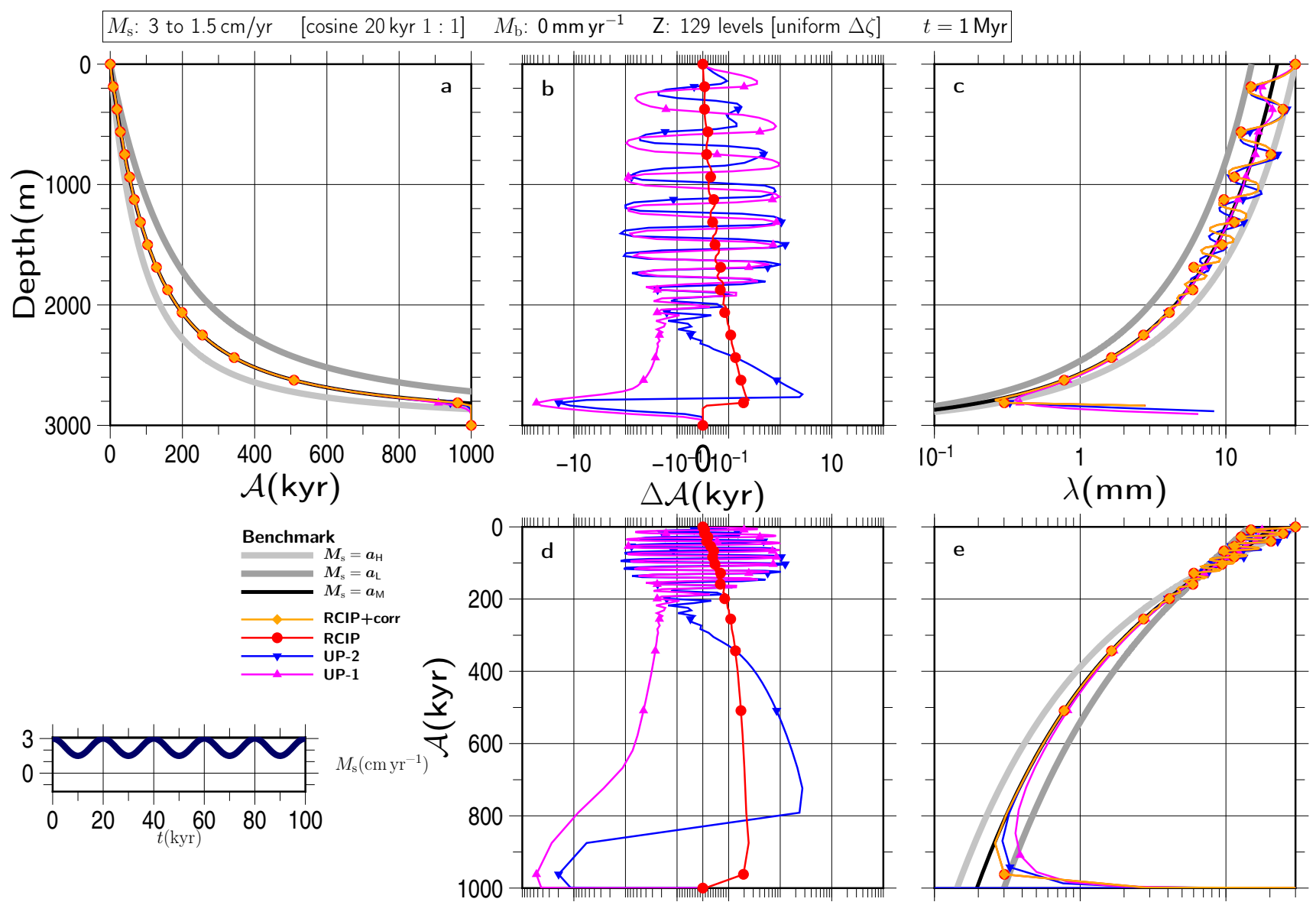

Figure S40 

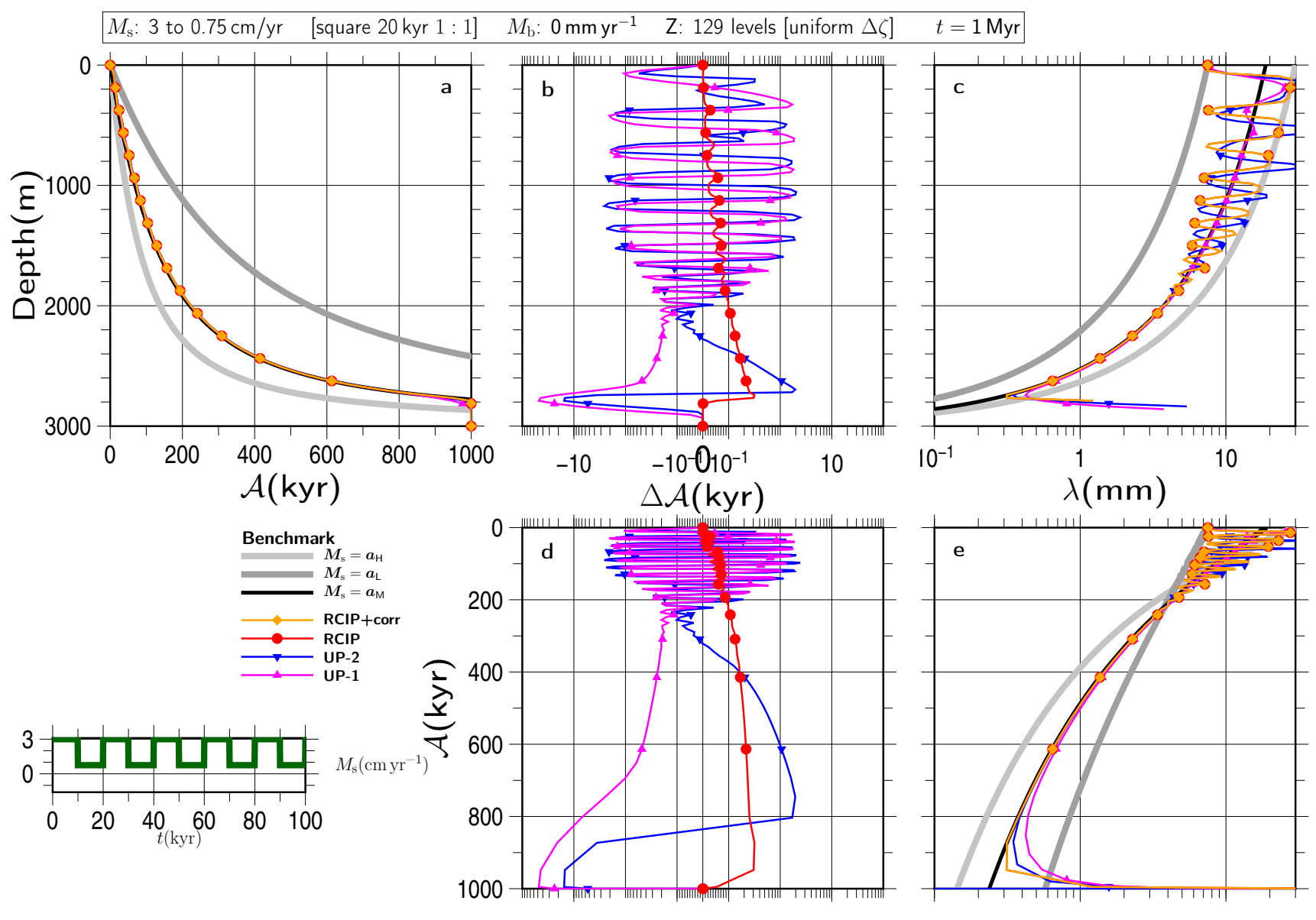

Figure S41
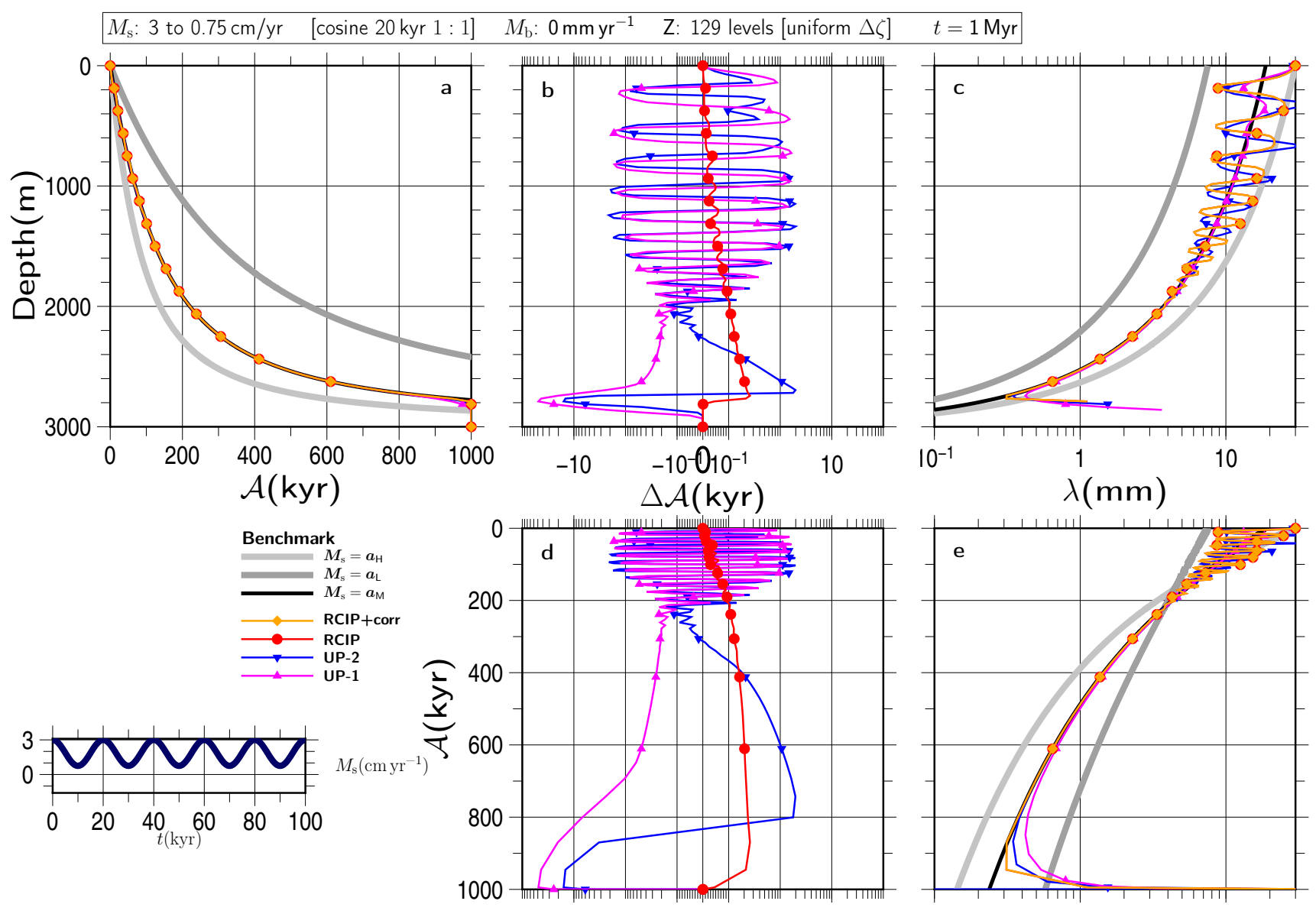

Figure S42 

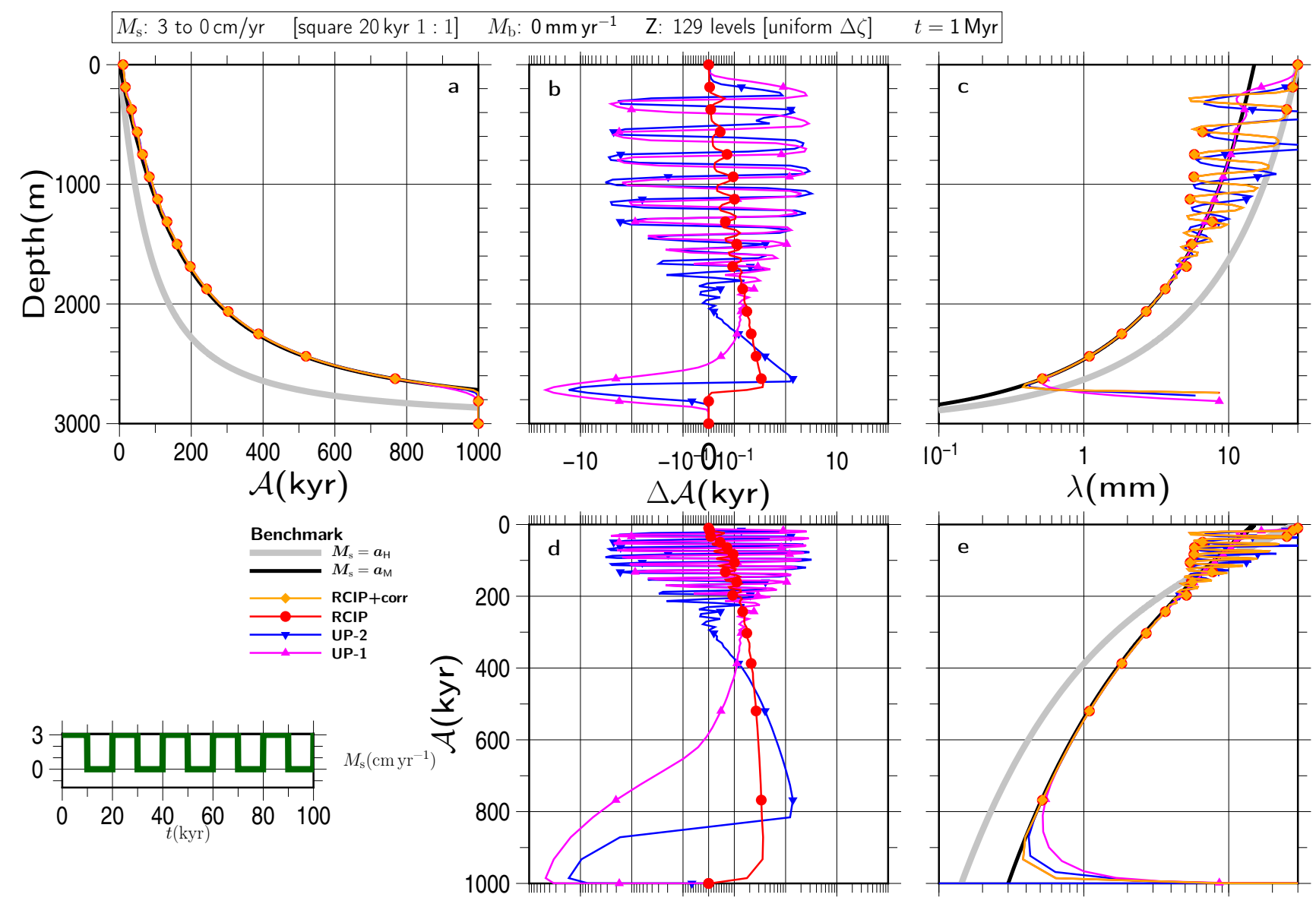

Figure S43
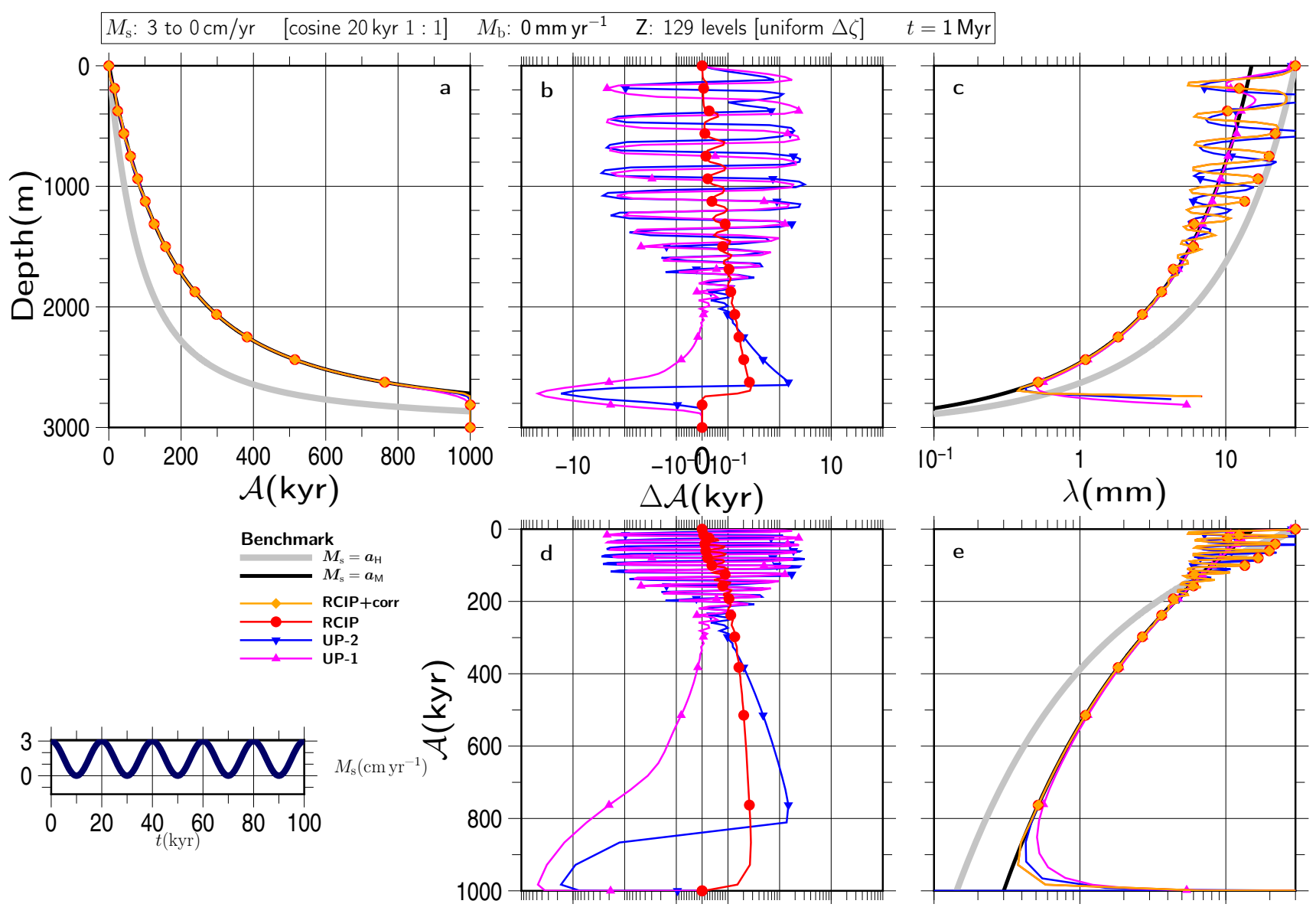

Figure S44 

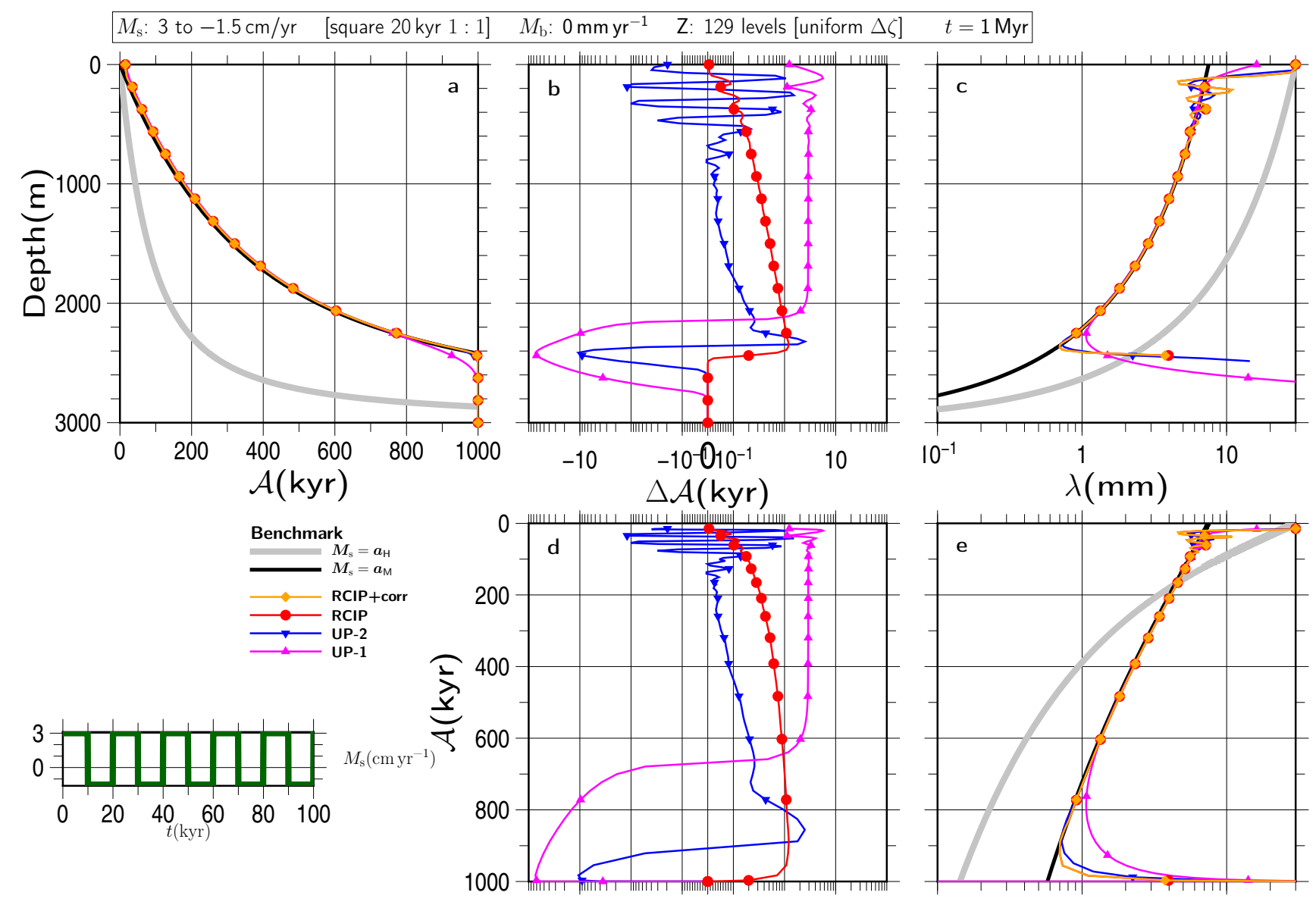

Figure S45
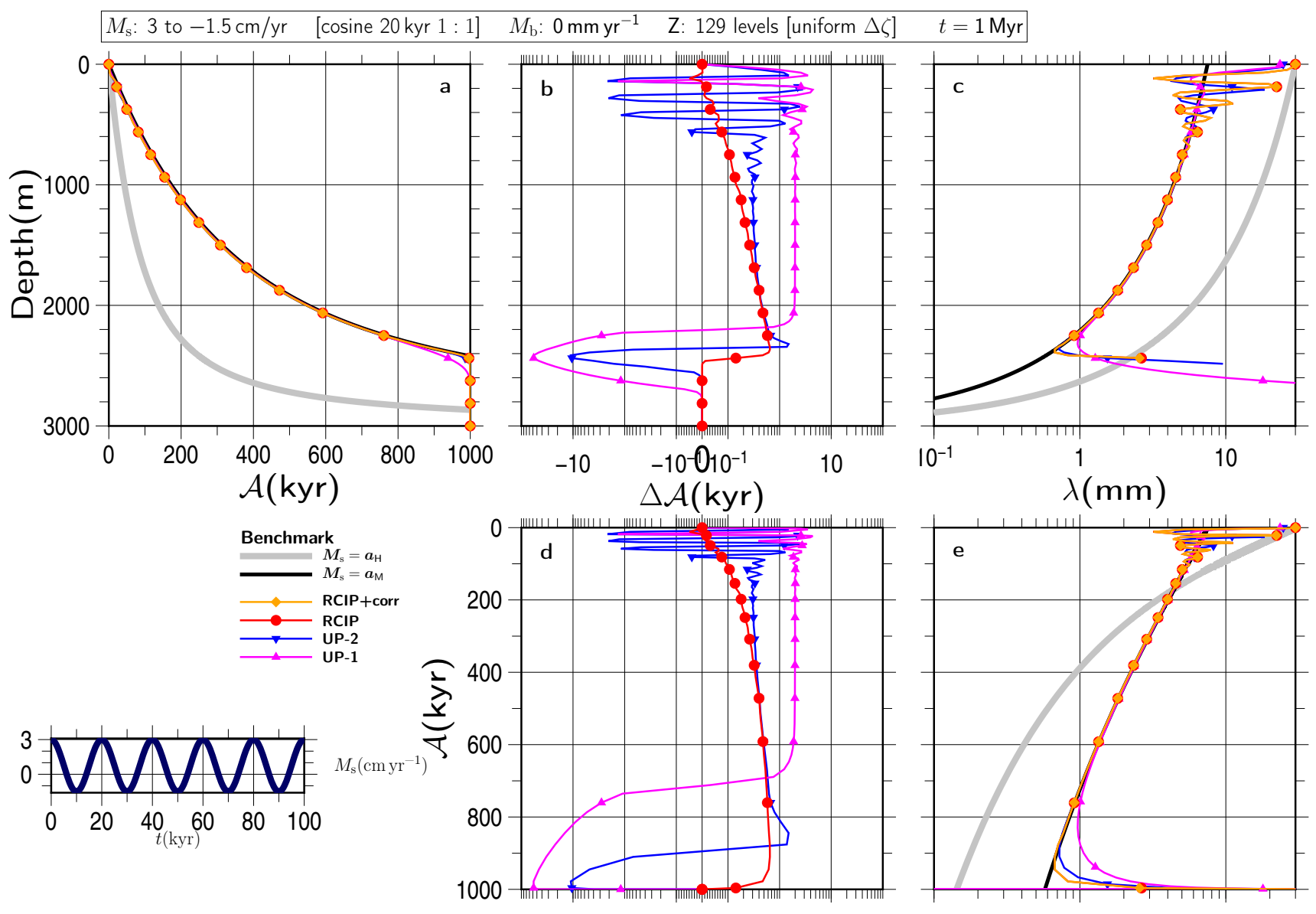

Figure S46 

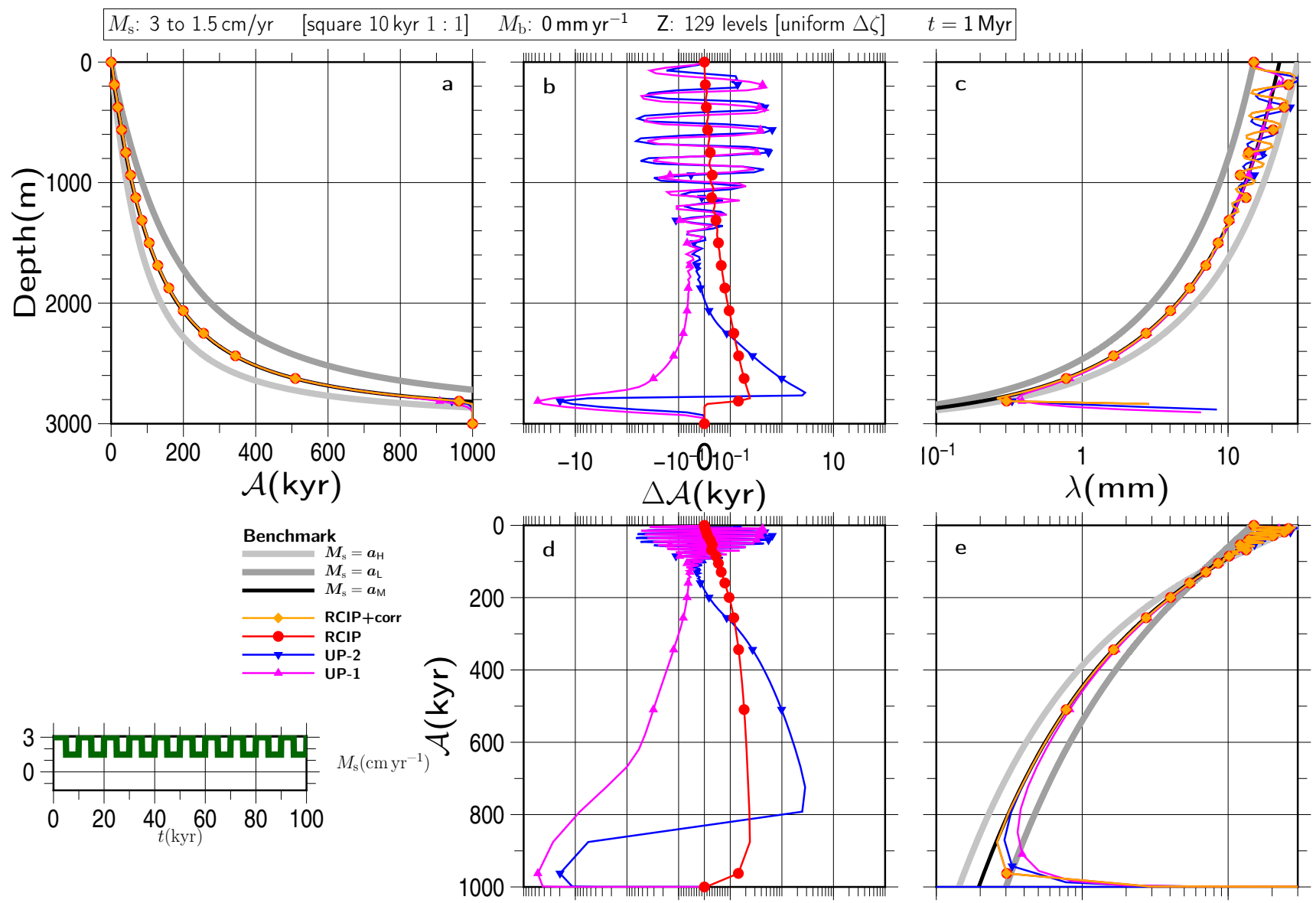

Figure S47
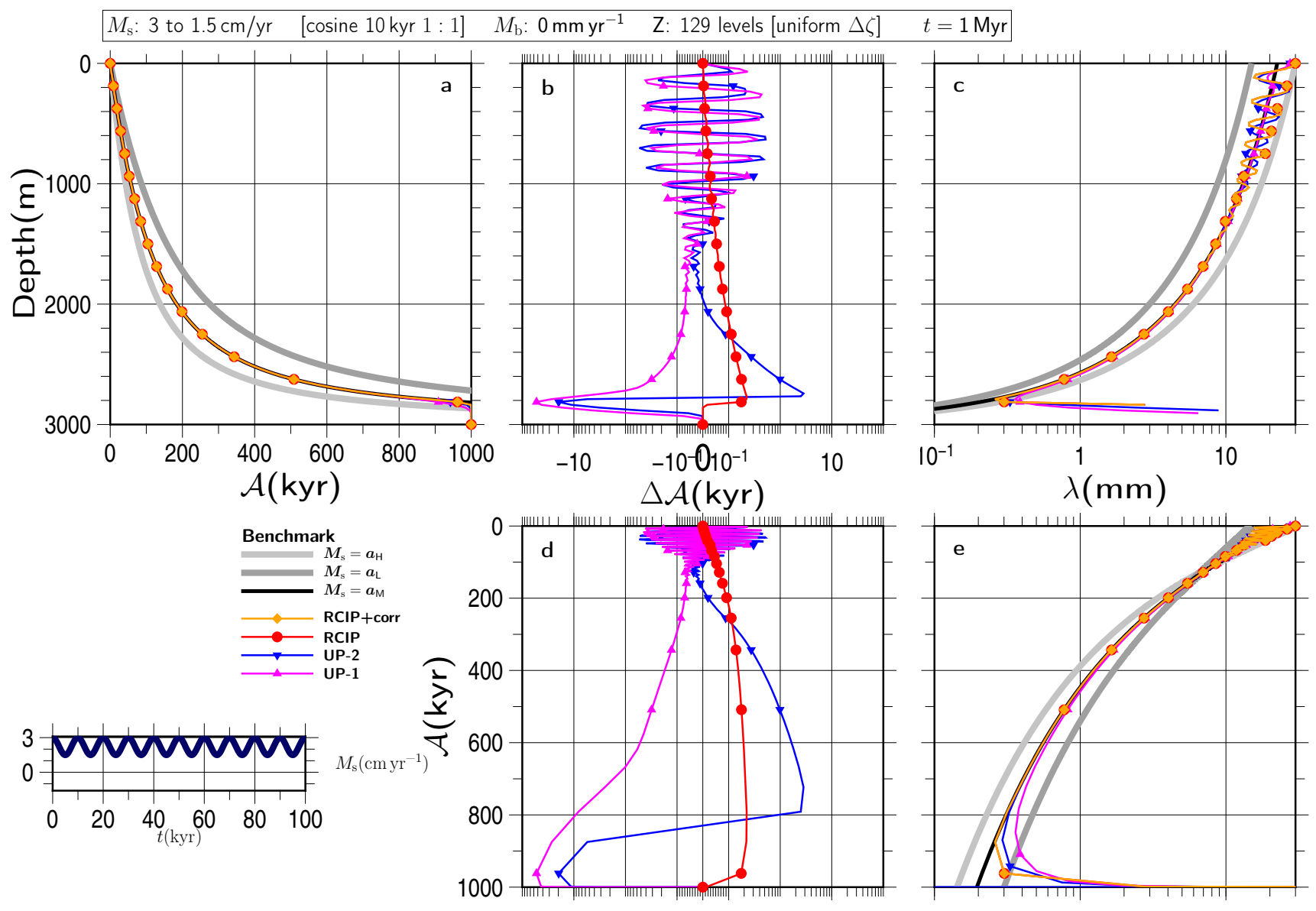

Figure S48 

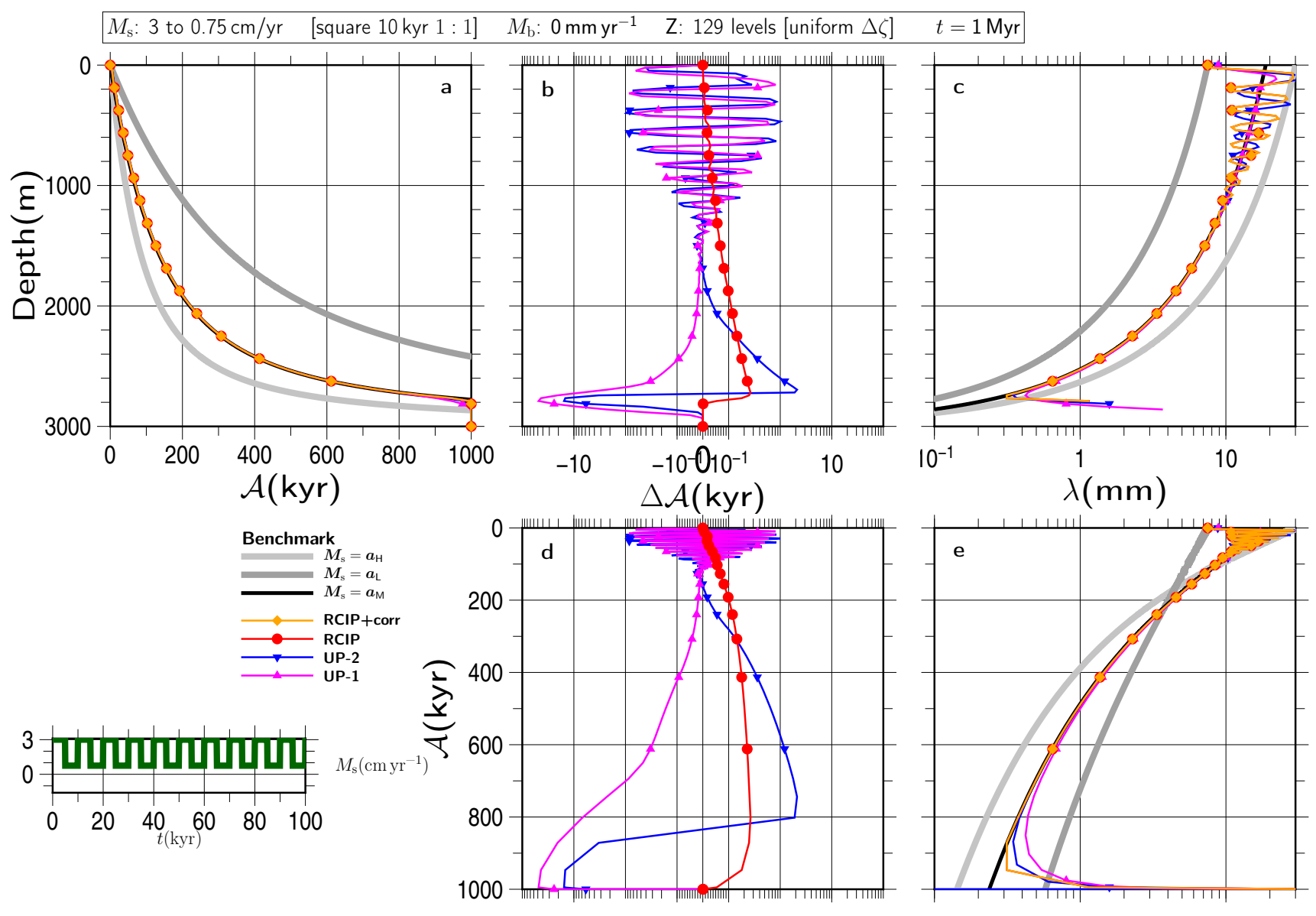

Figure S49
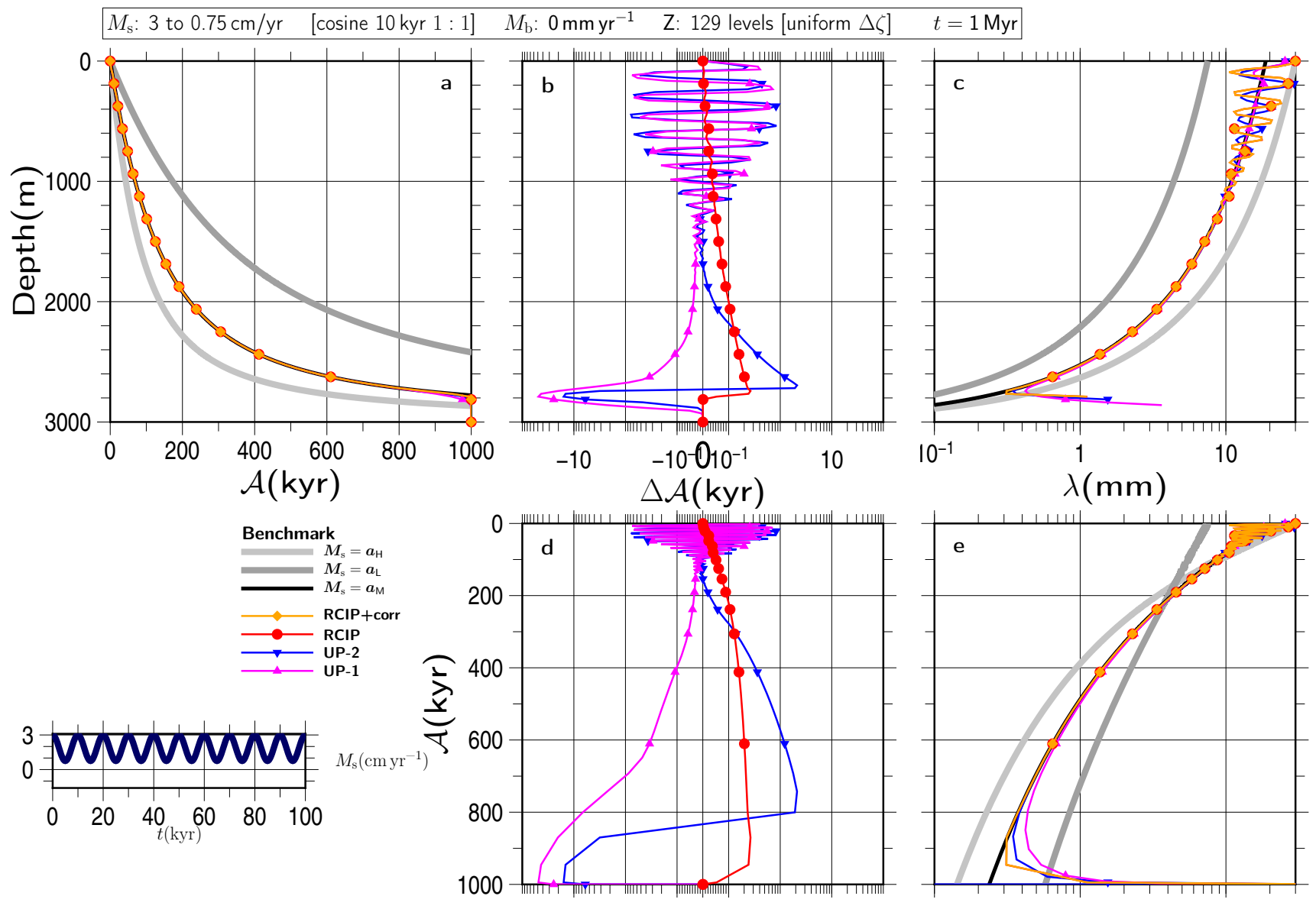

Figure S50 

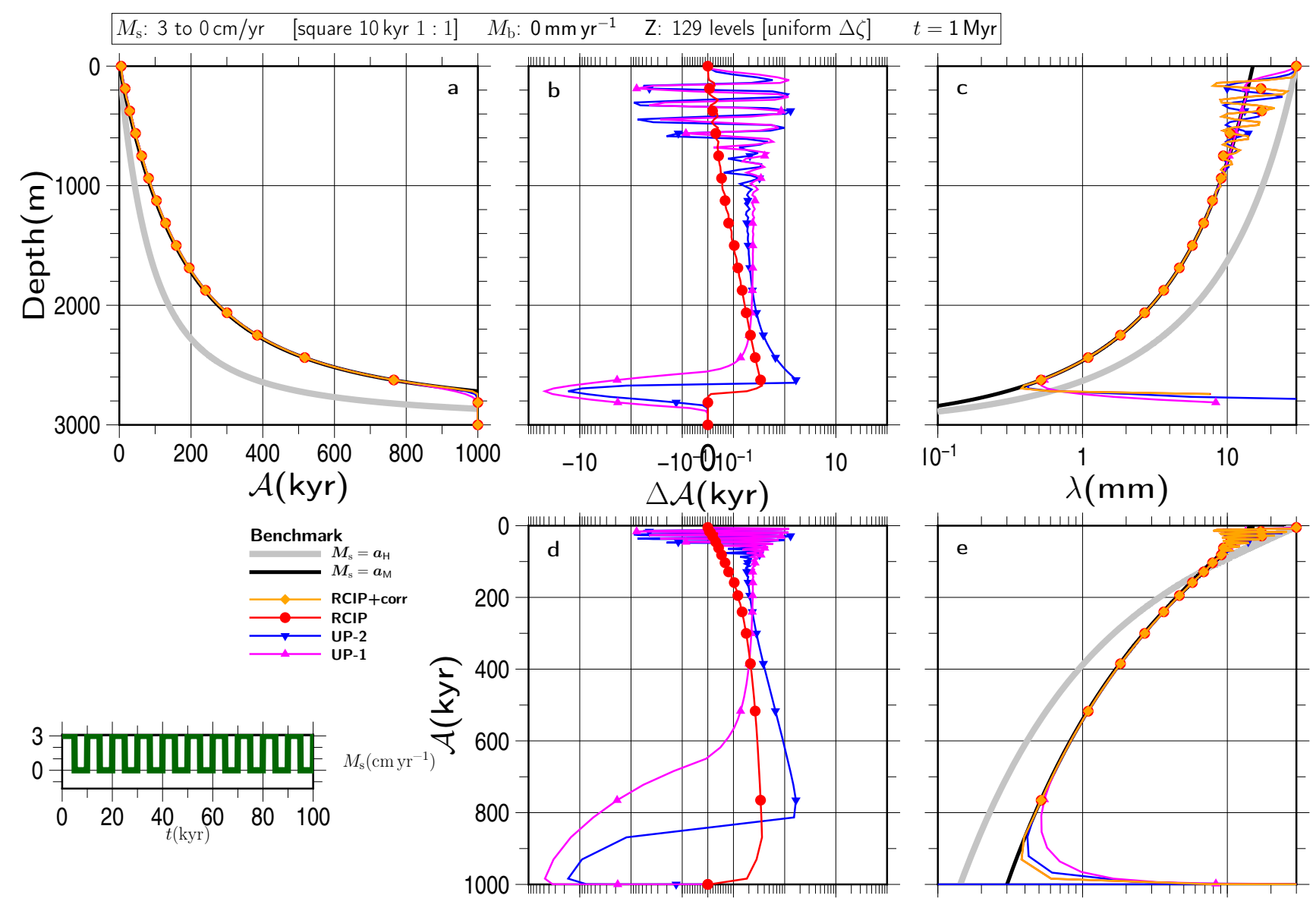

Figure S51
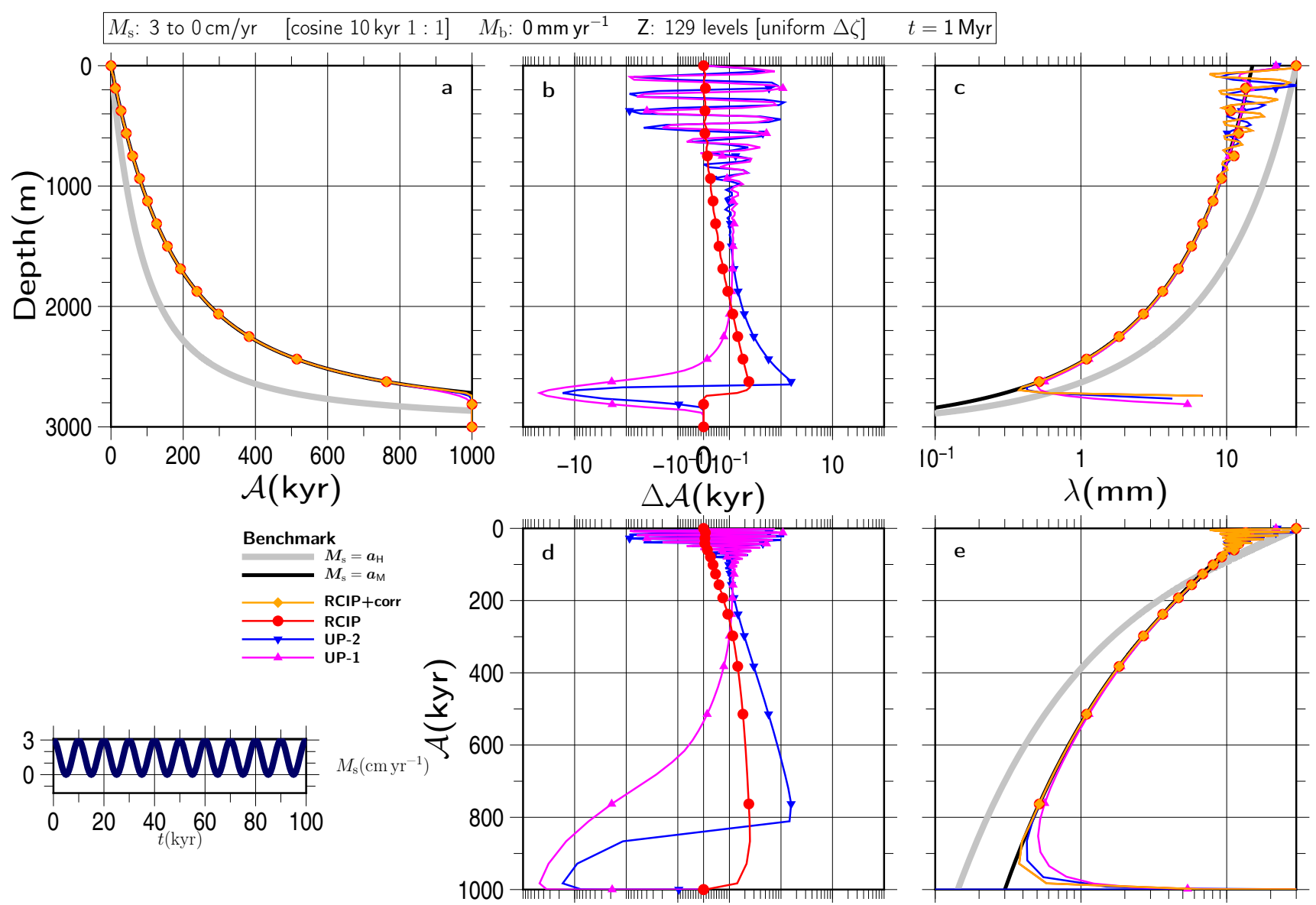

Figure S52 

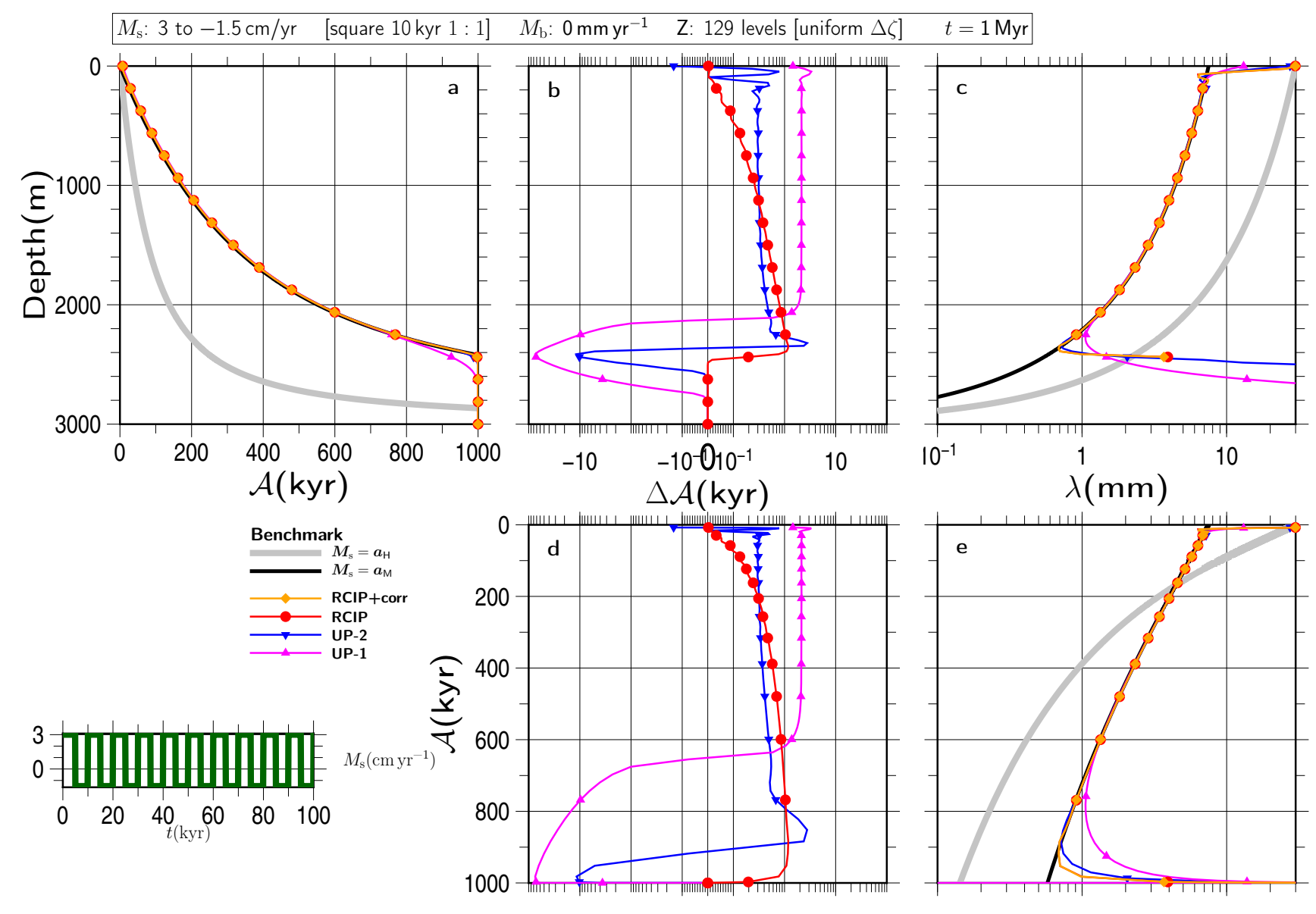

Figure S53
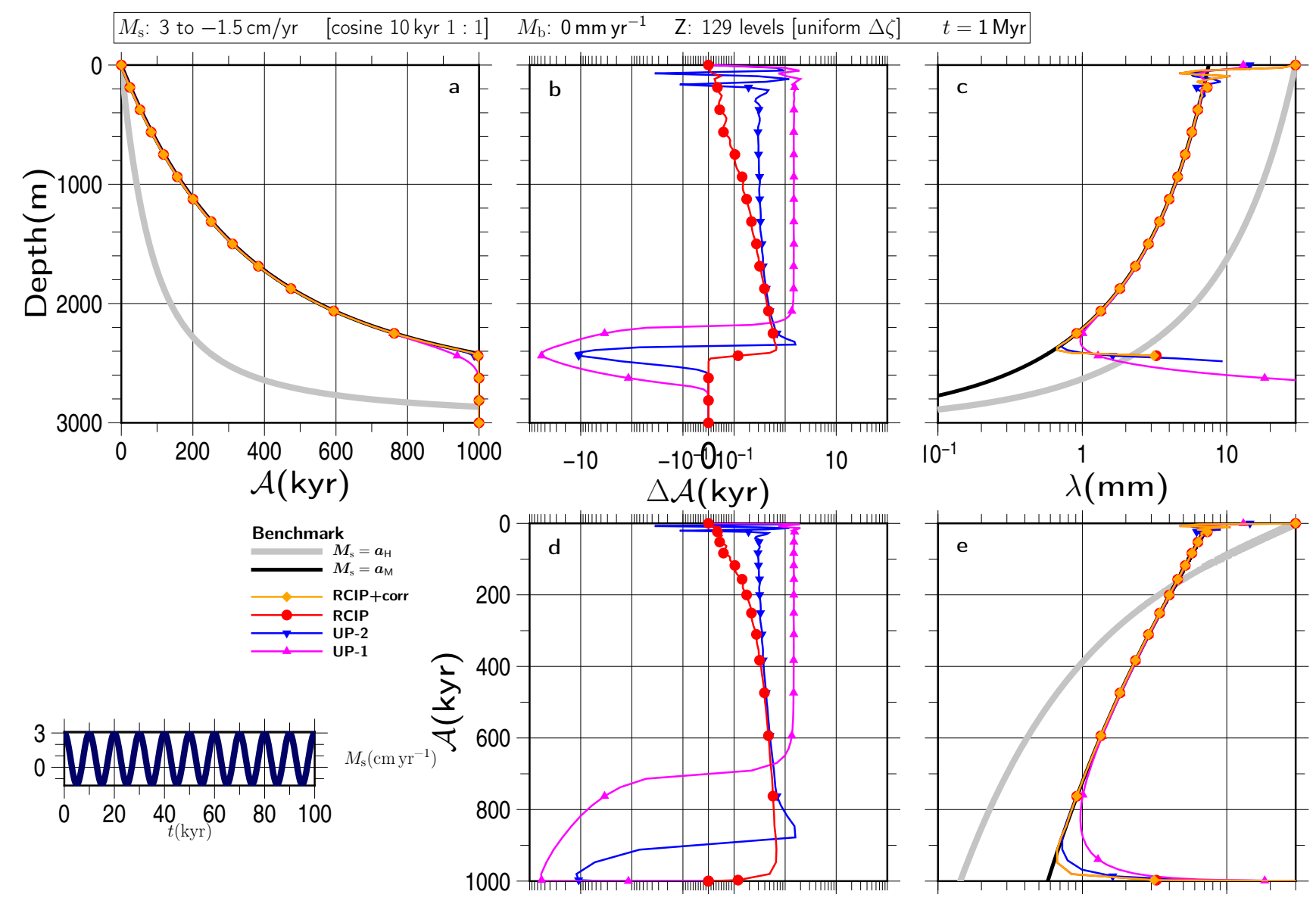

Figure S54 

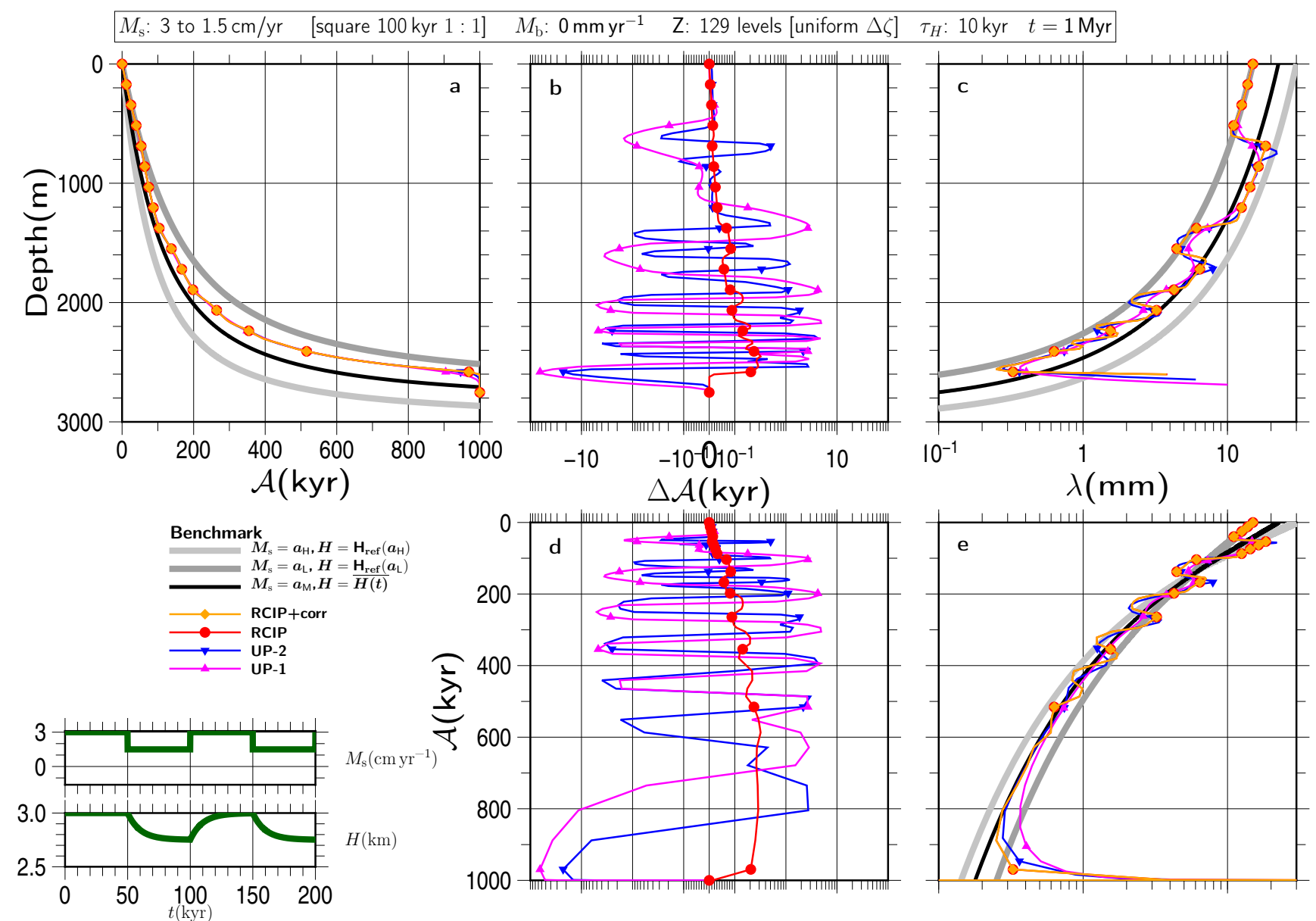

Figure S55
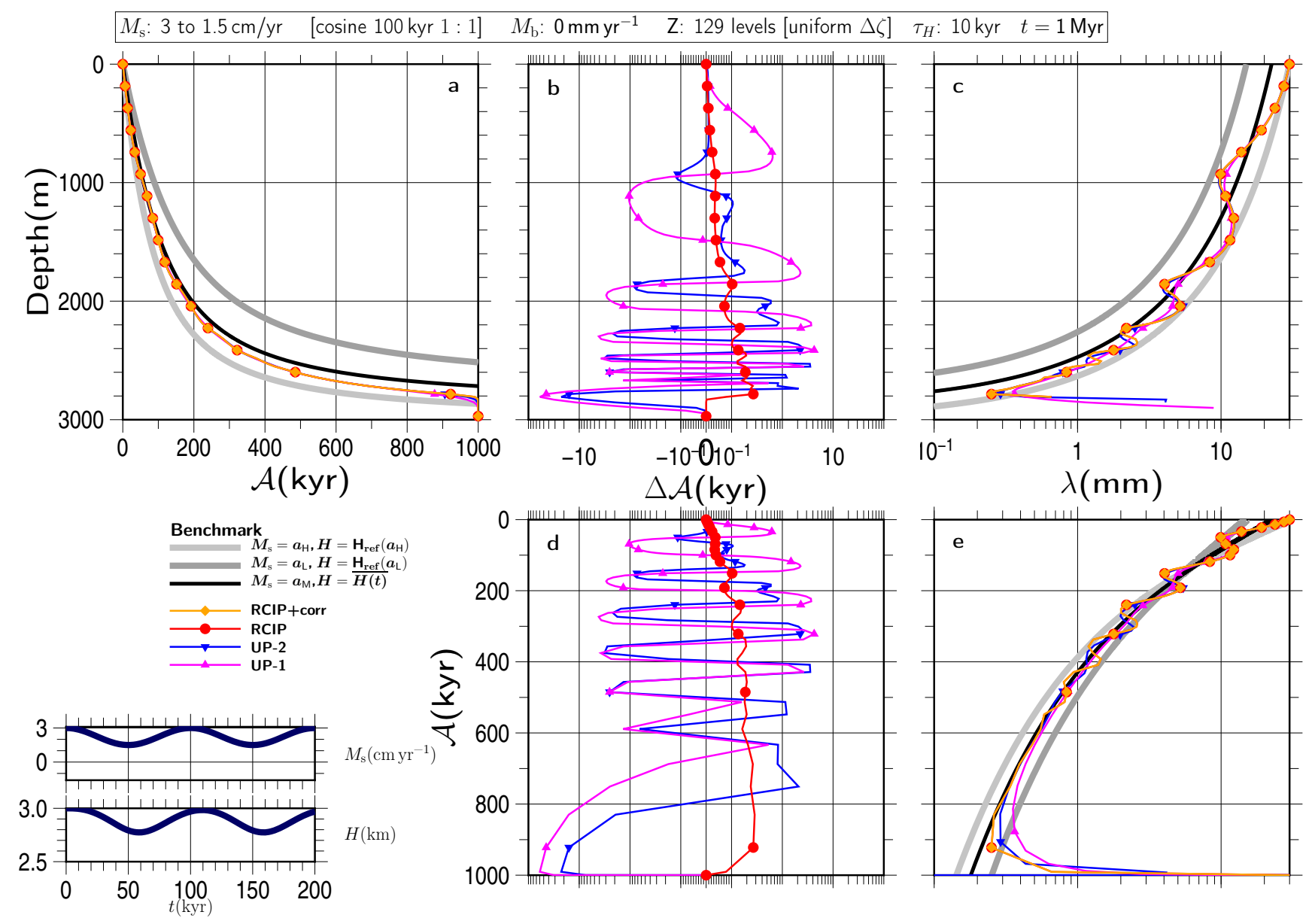

Figure S56 

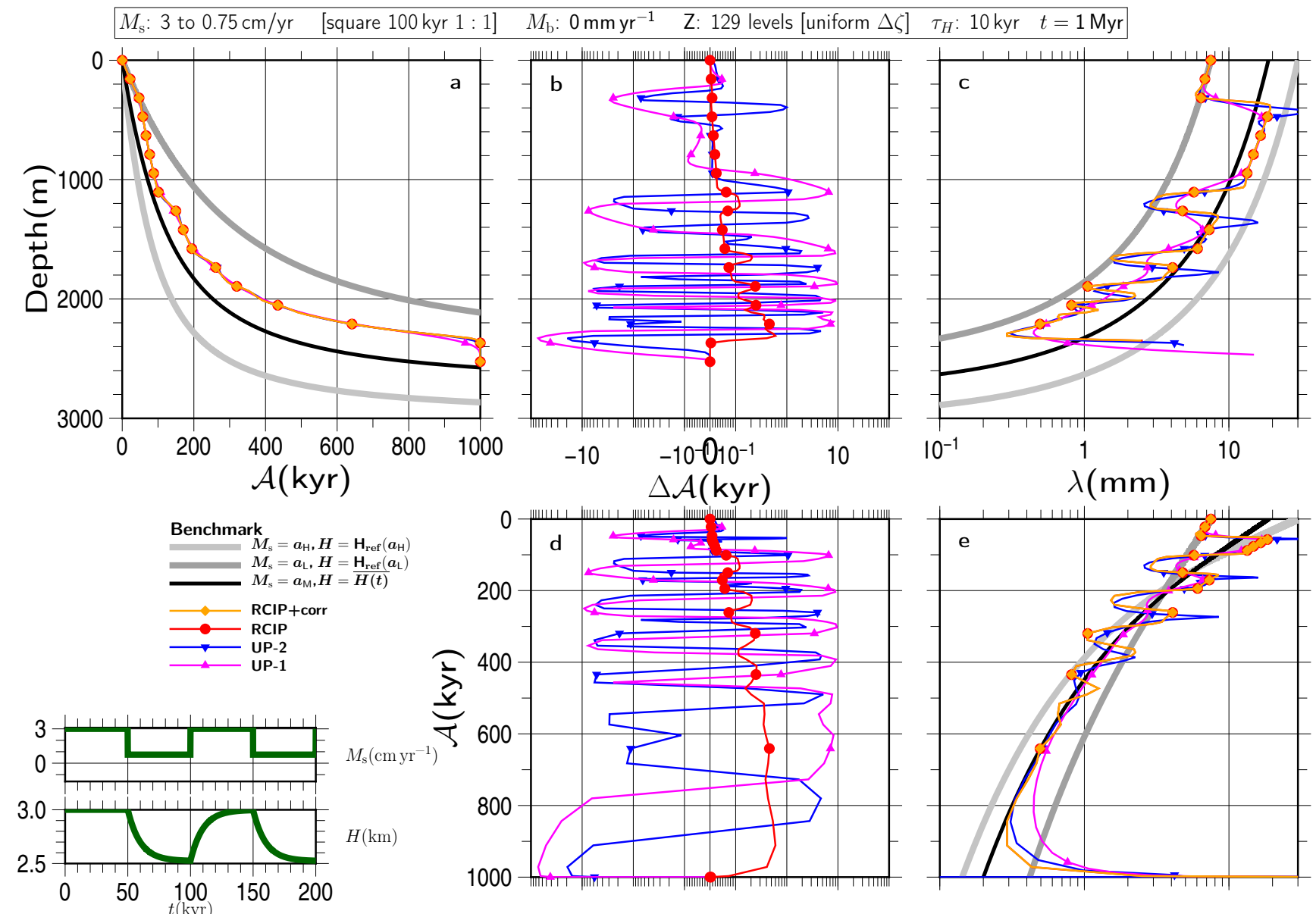

Figure S57
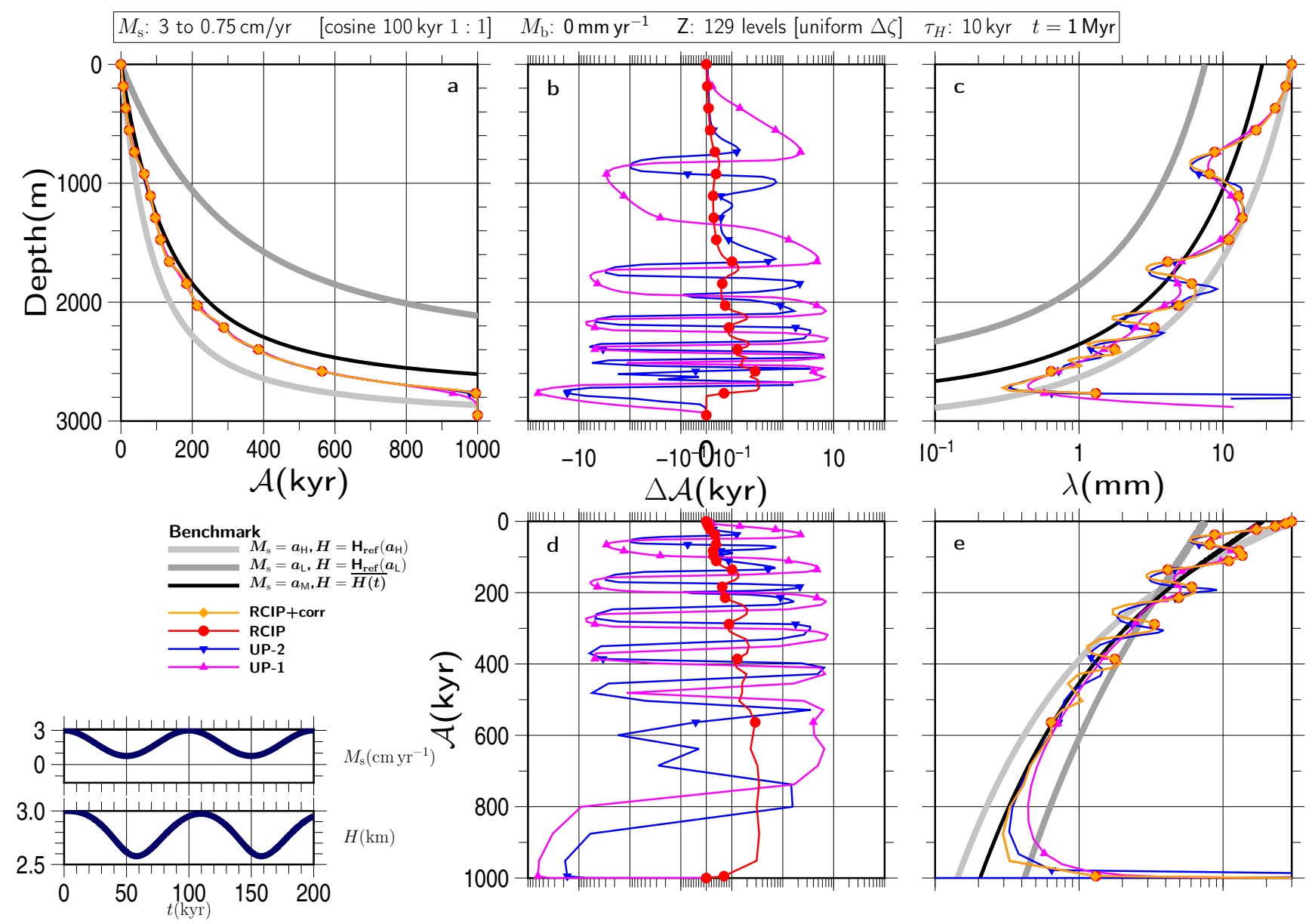

Figure S58 

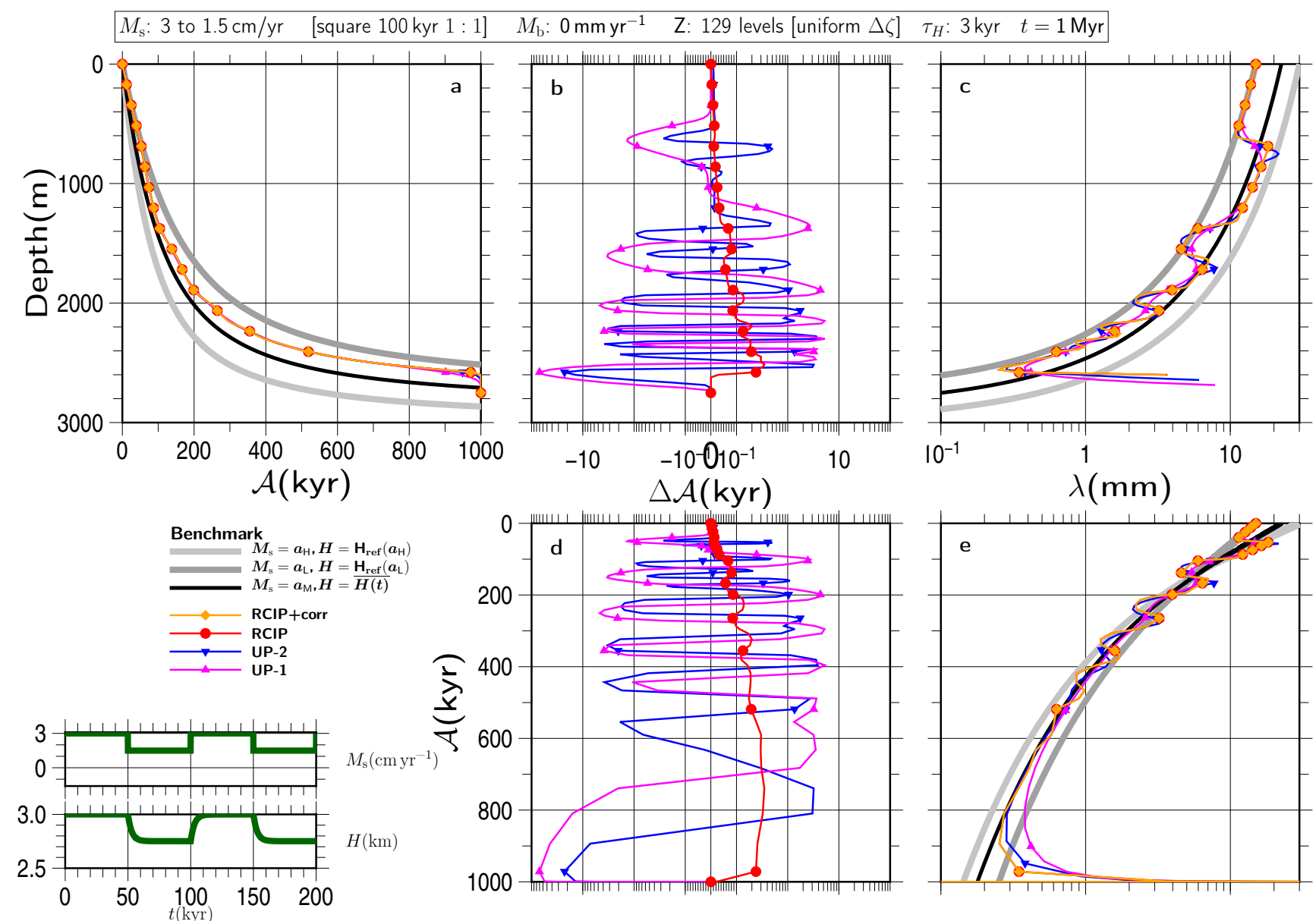

Figure S59
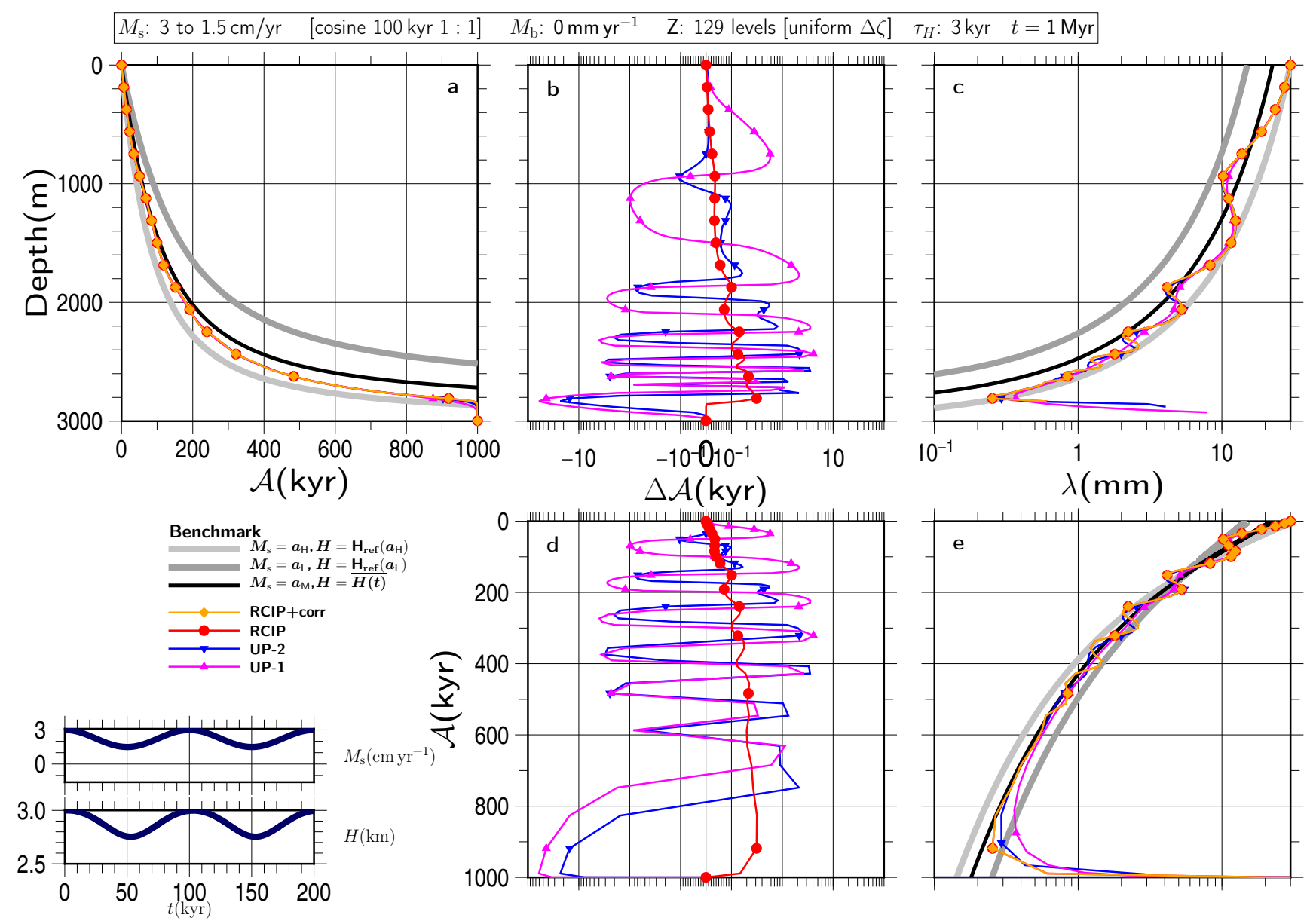

Figure S60 

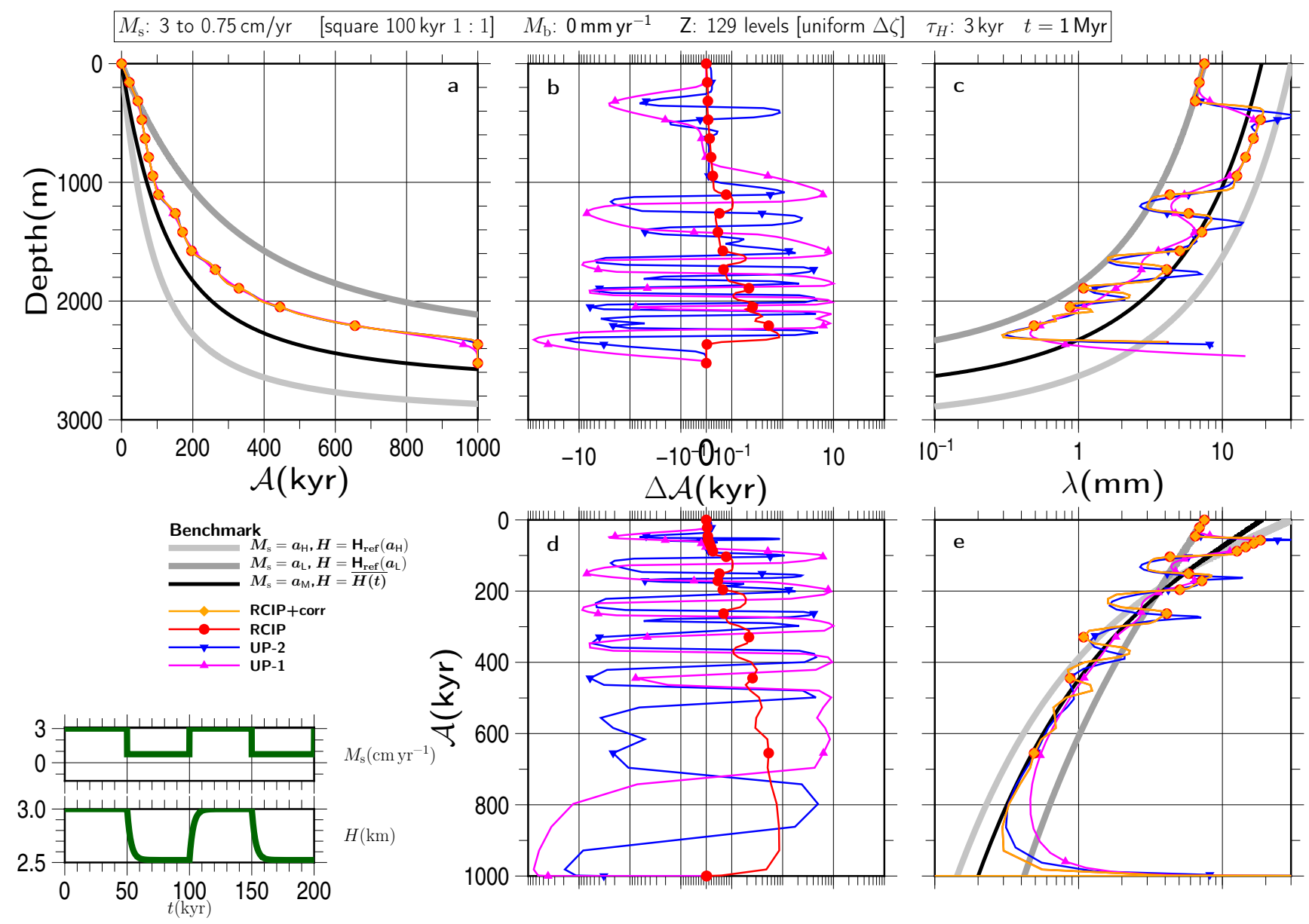

Figure S61
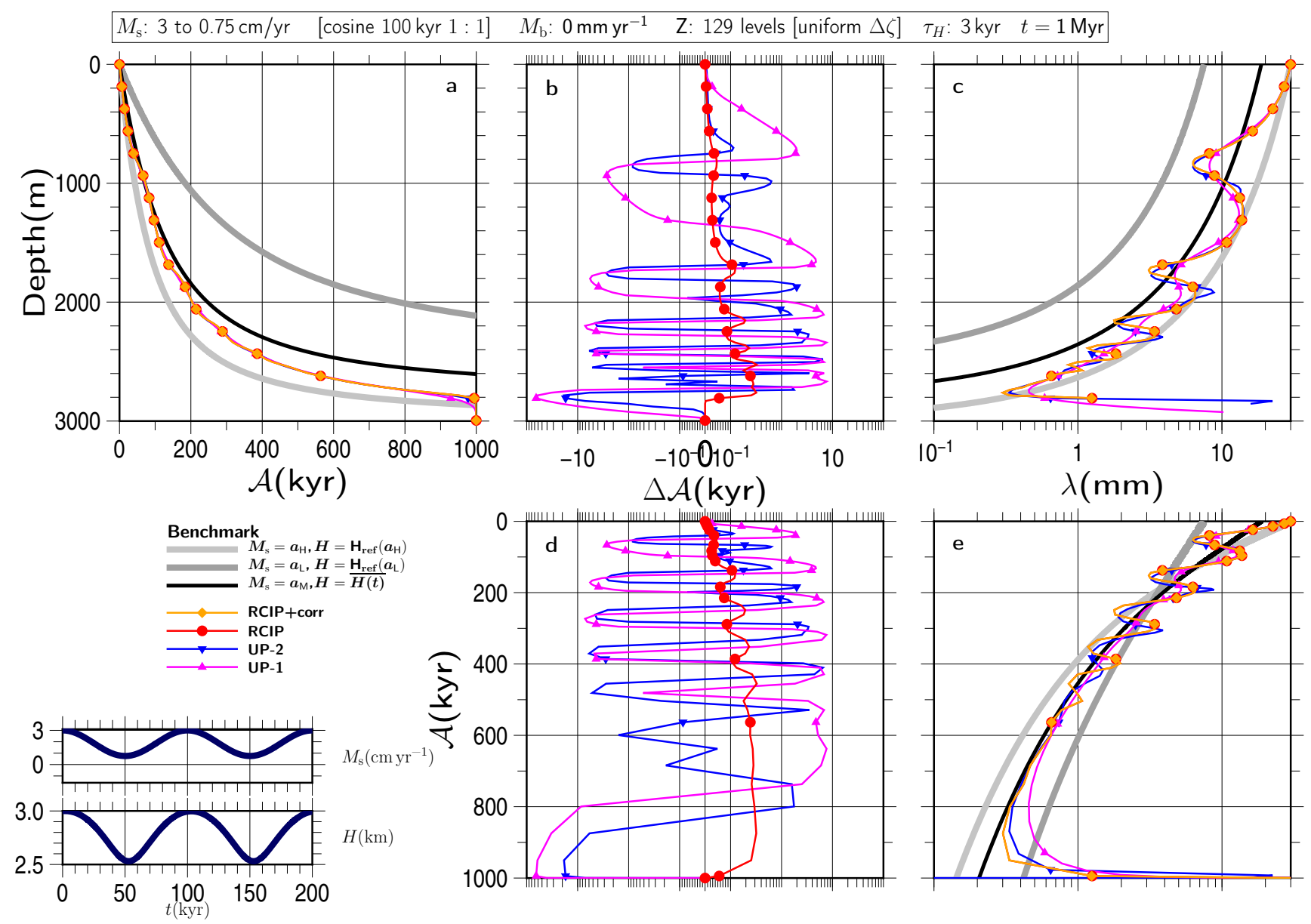

Figure S62 

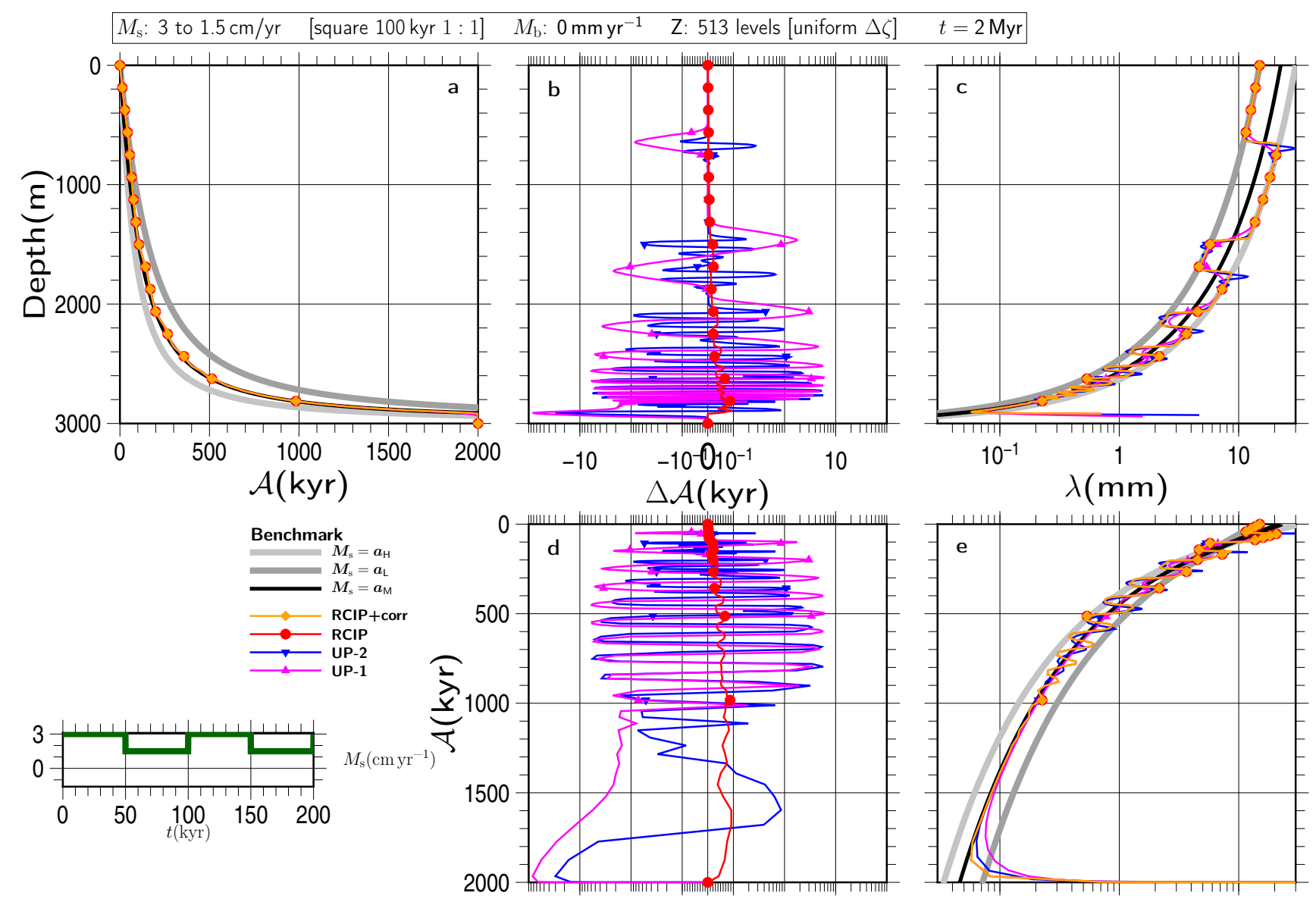

Figure S63
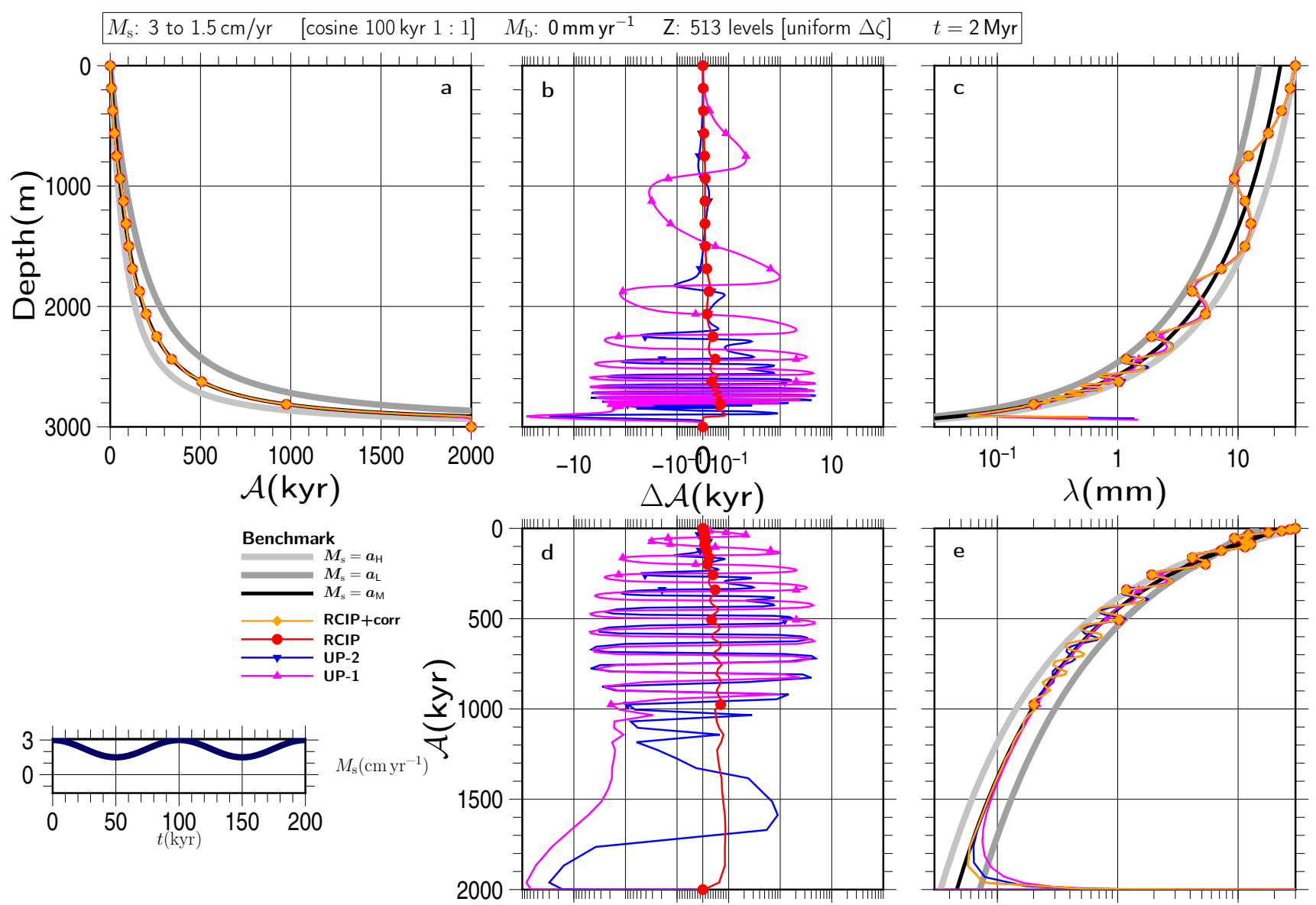

Figure S64 

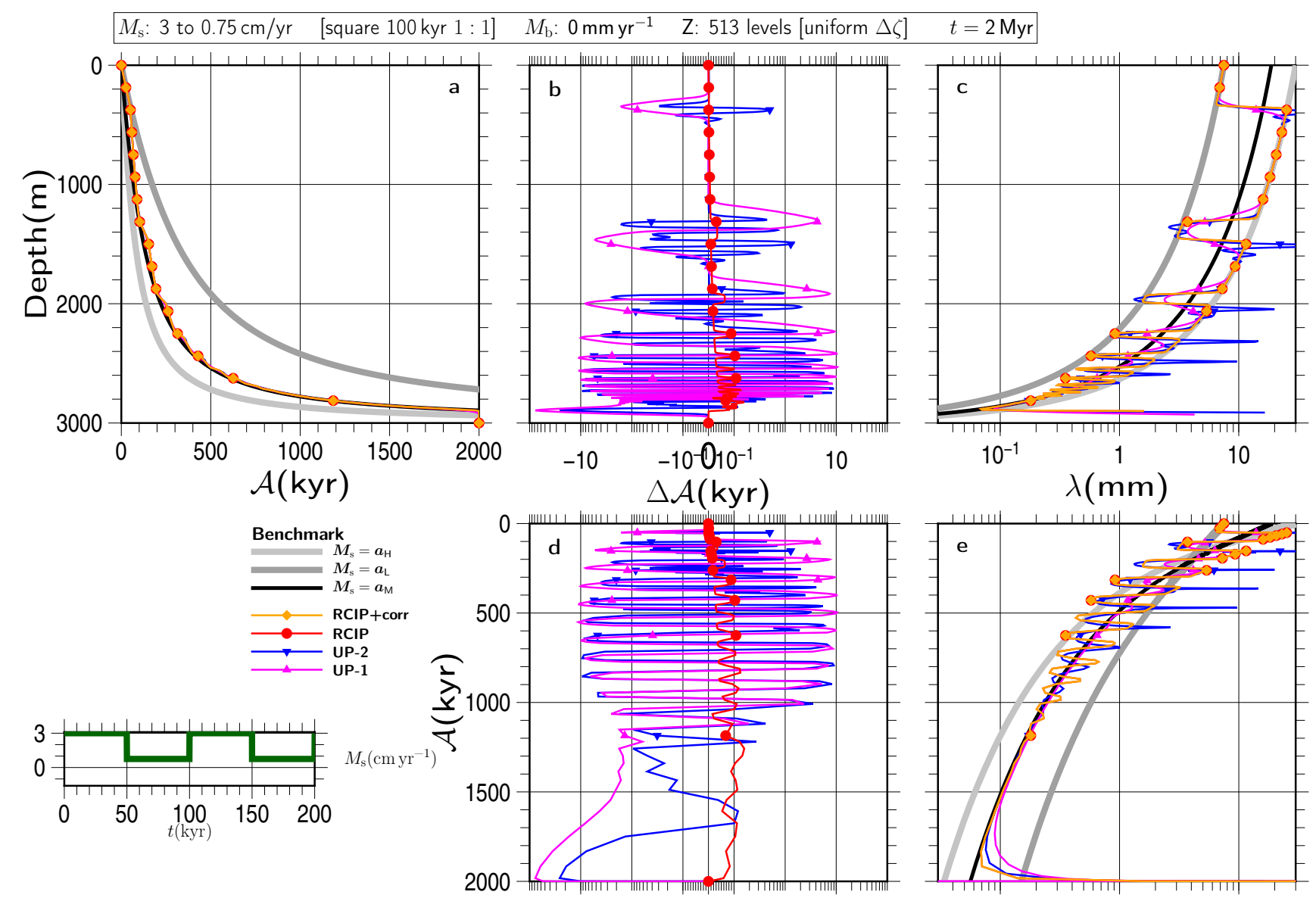

Figure S65
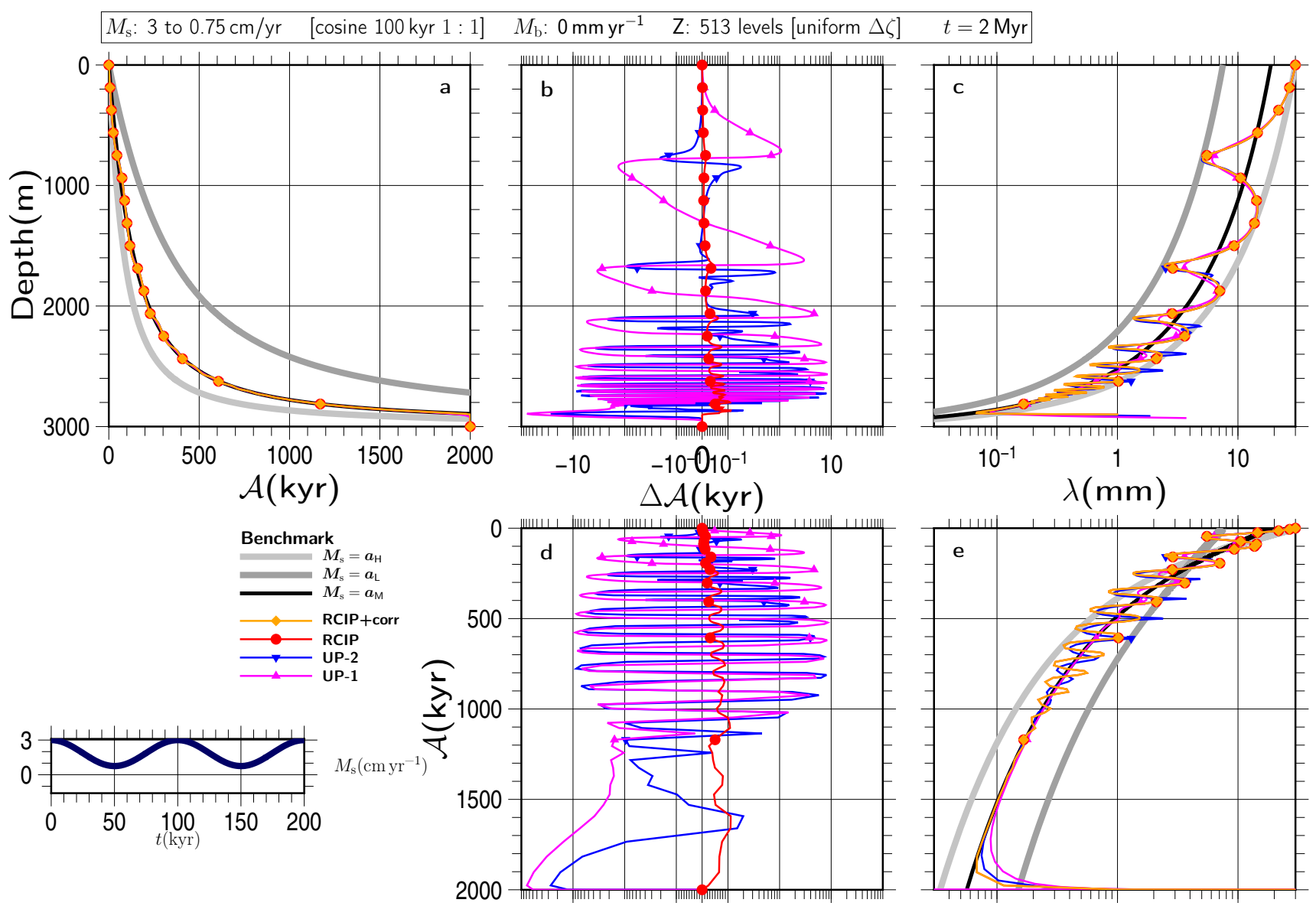

Figure S66 

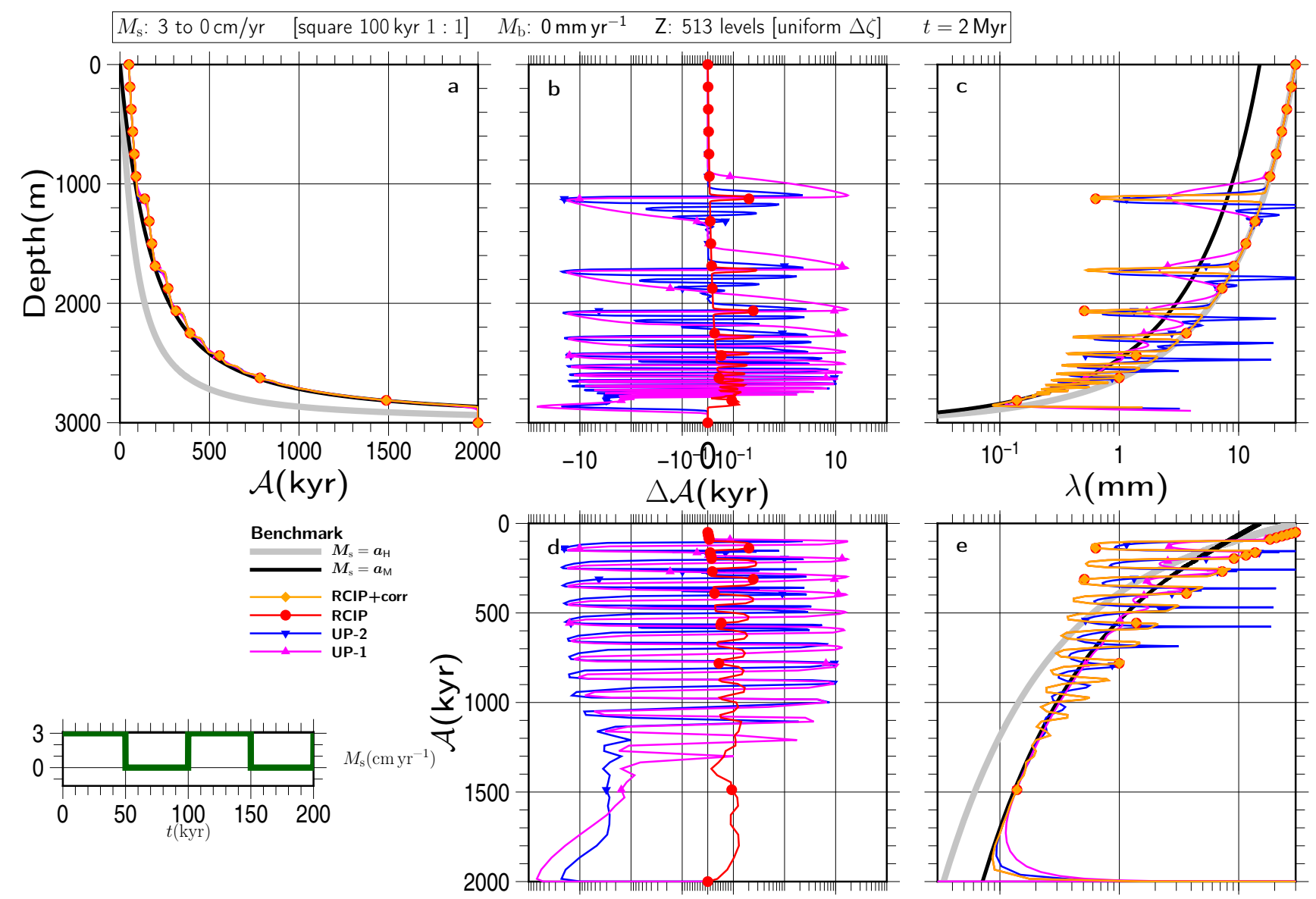

Figure S67
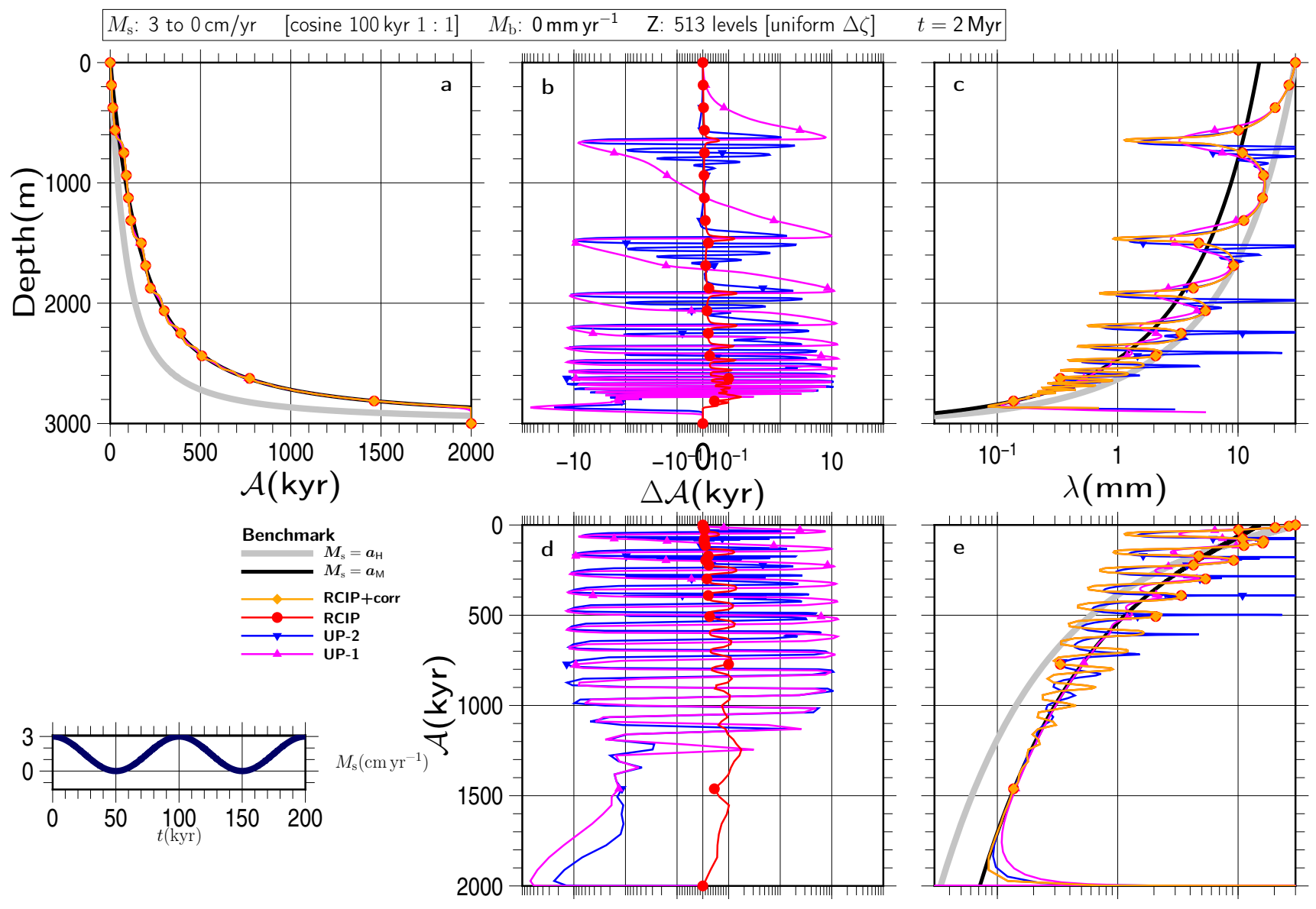

Figure S68 

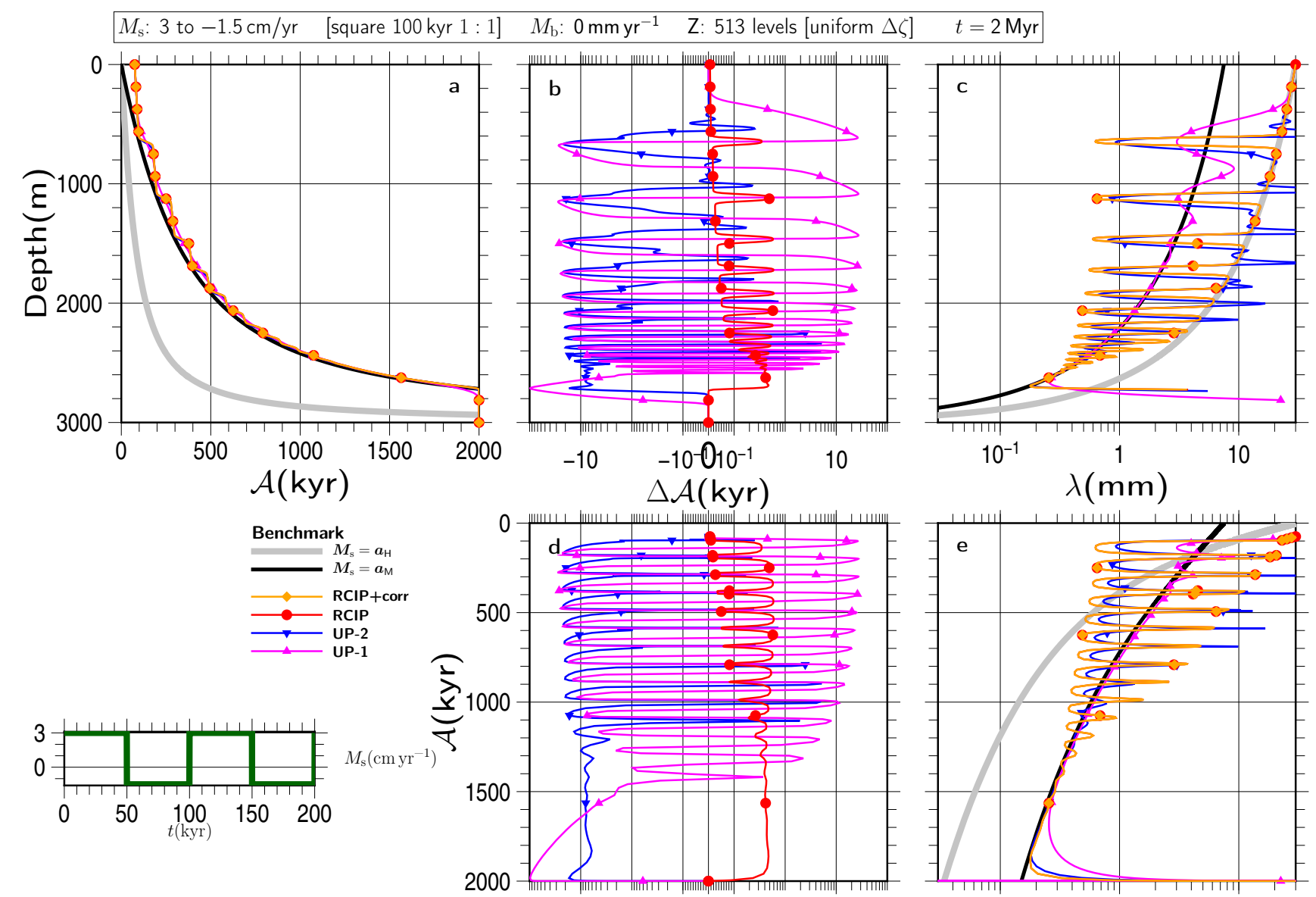

Figure S69
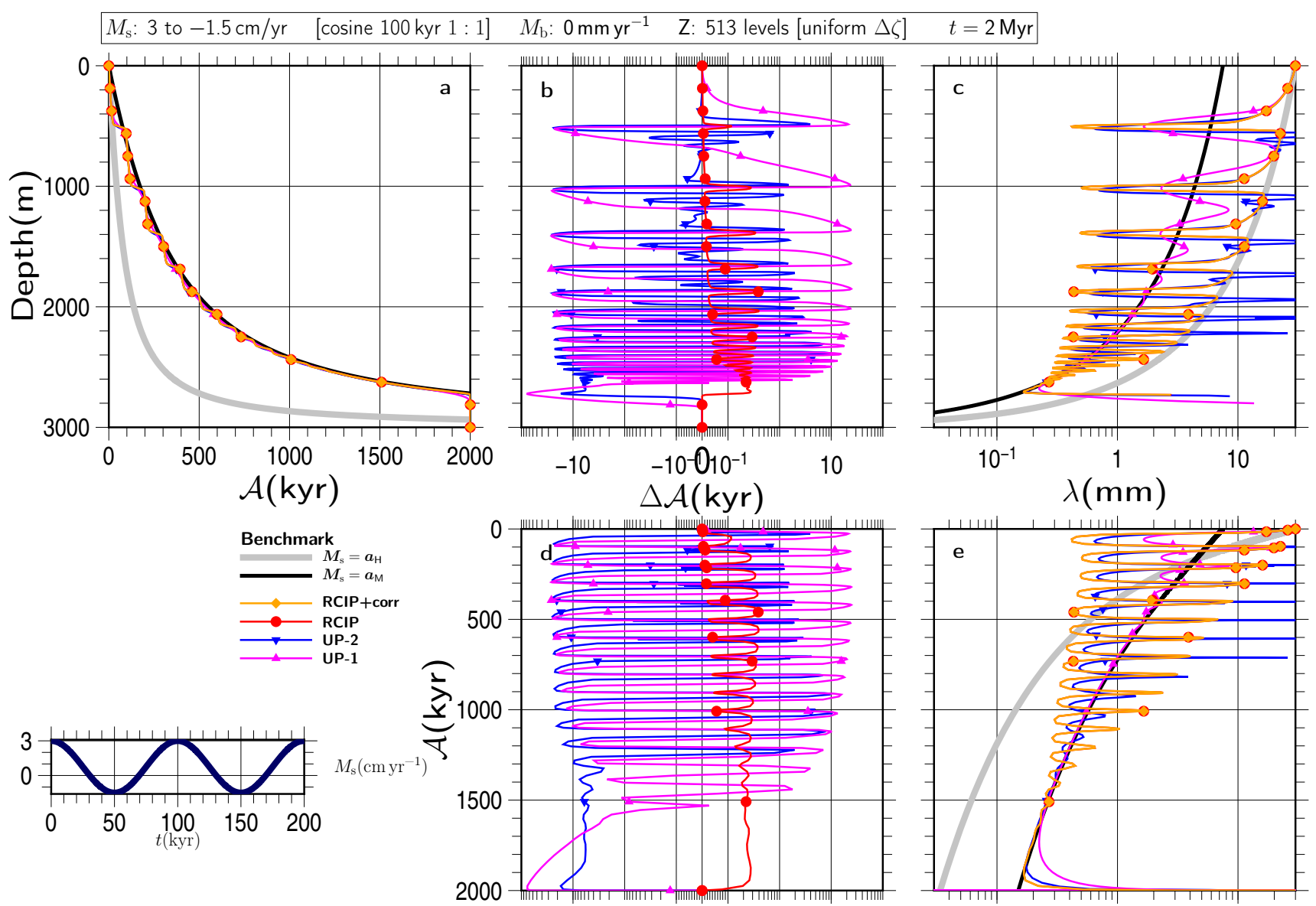

Figure S70 

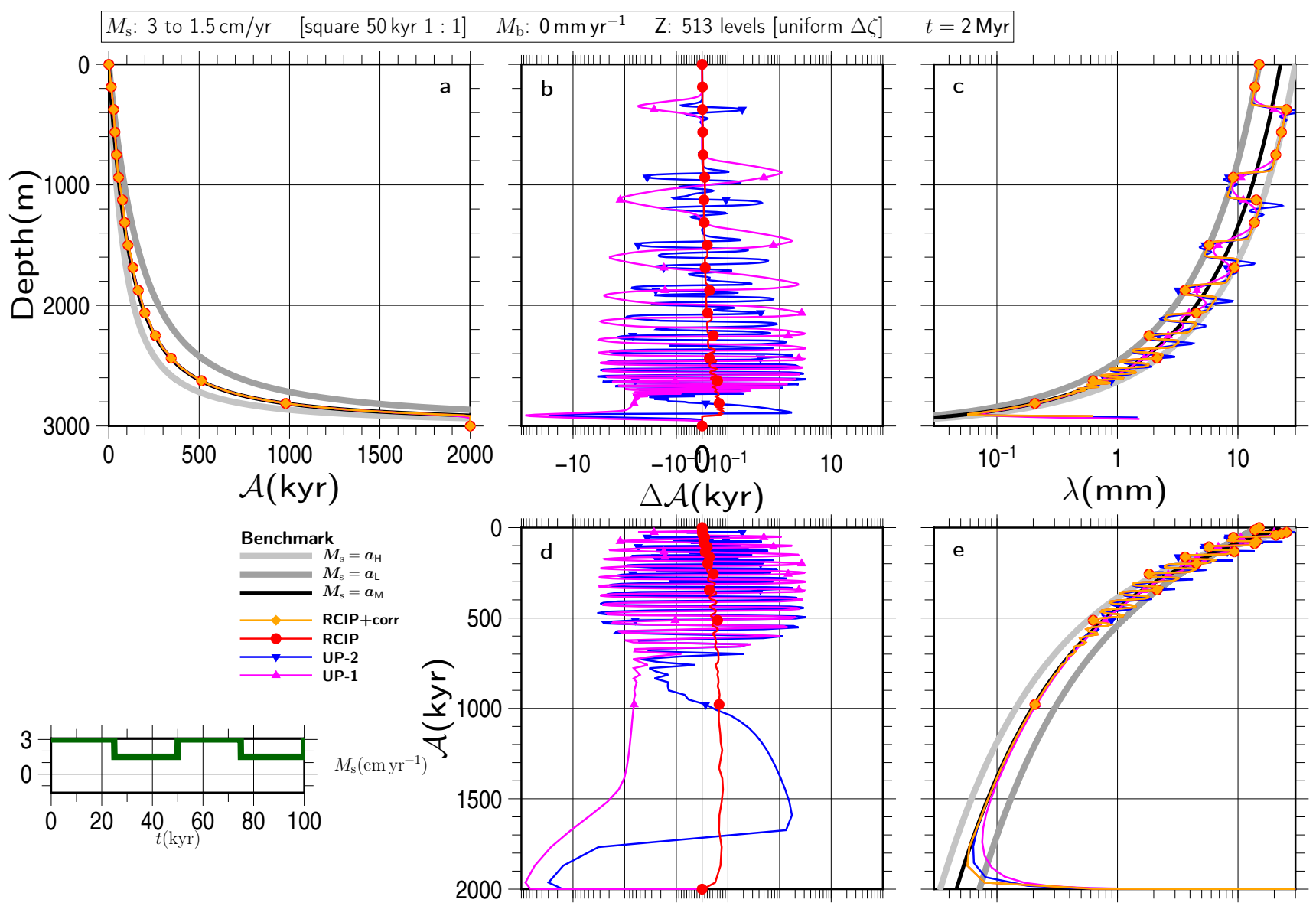

Figure S71
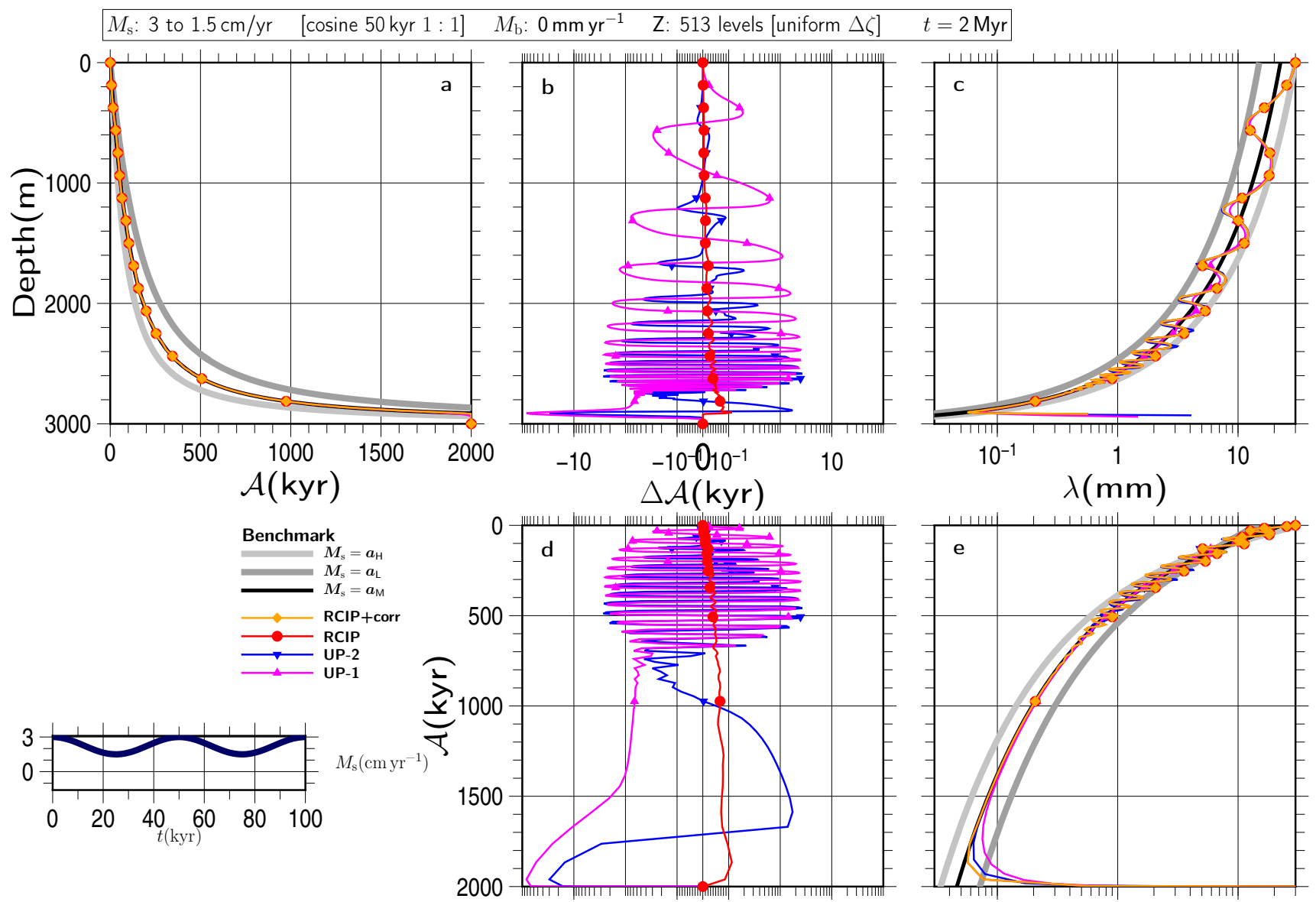

Figure S72 

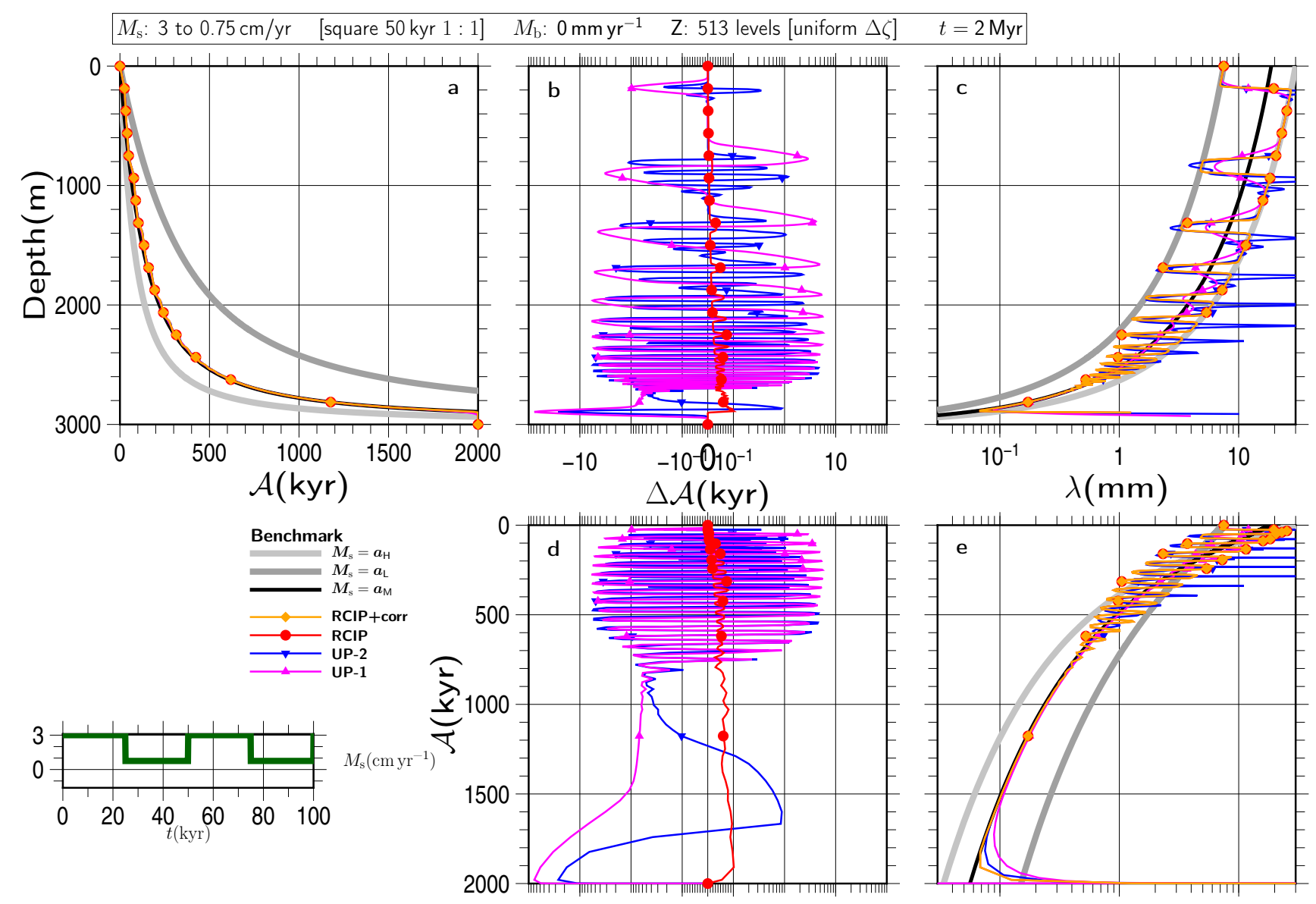

Figure S73
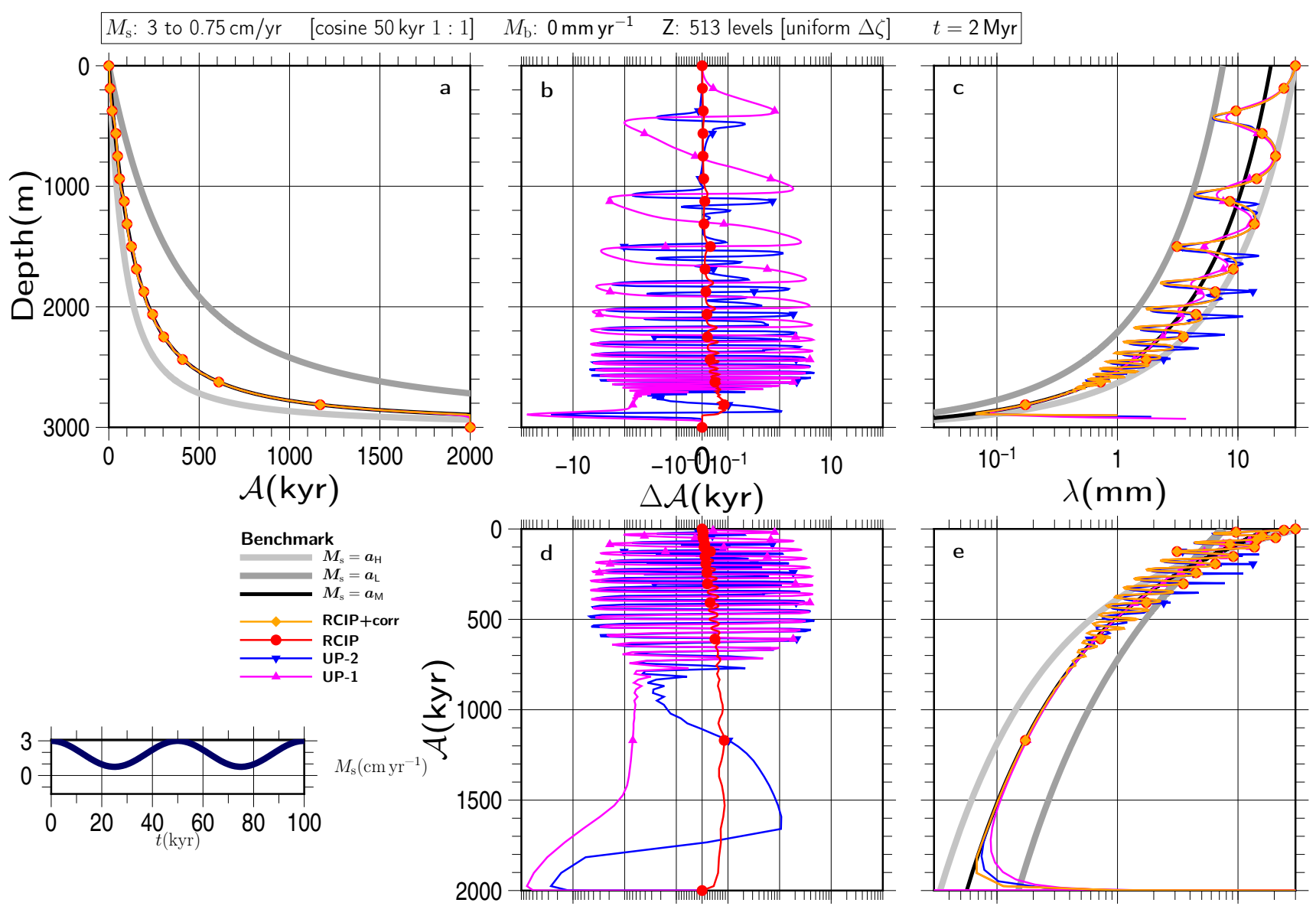

Figure S74 

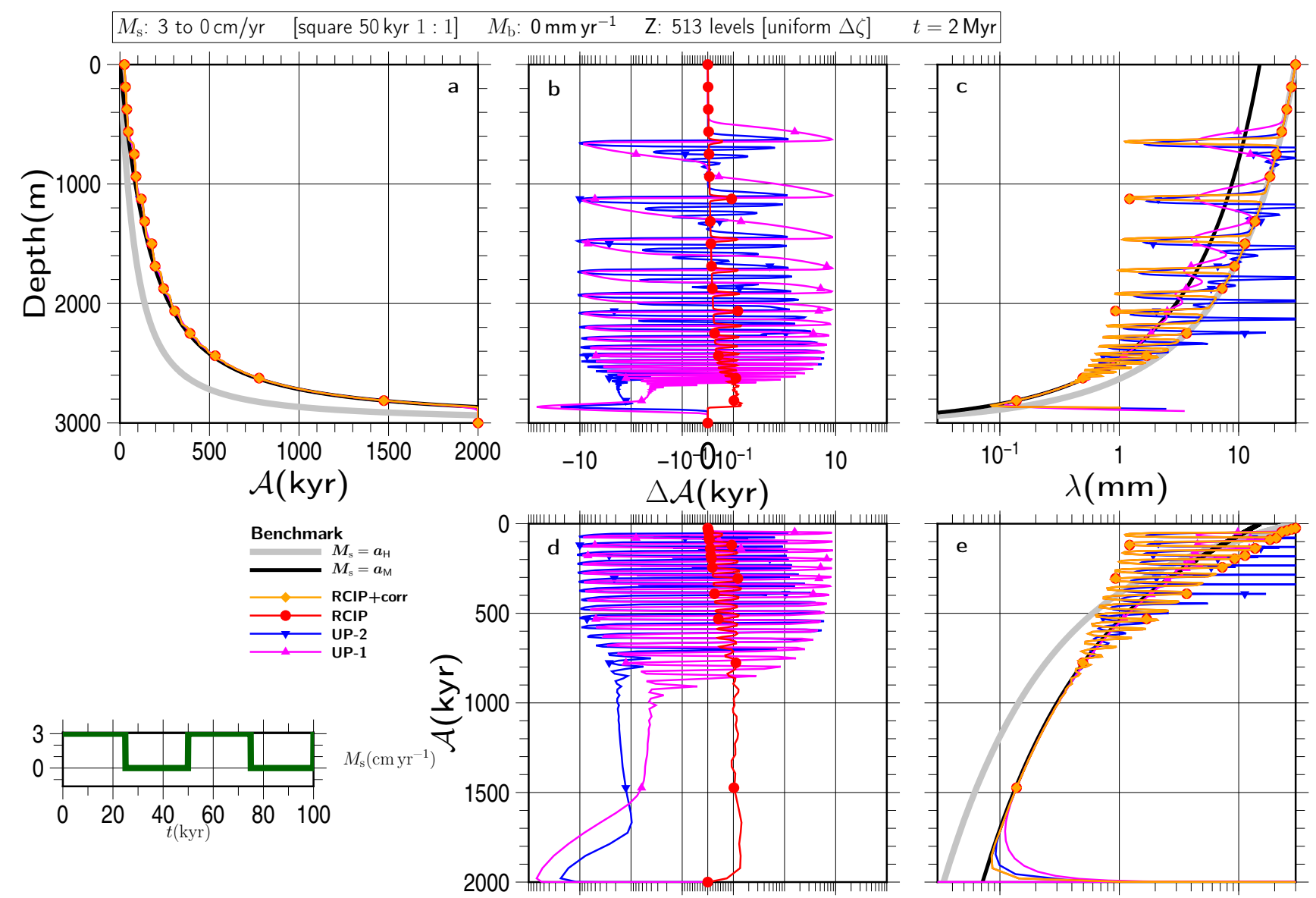

Figure S75
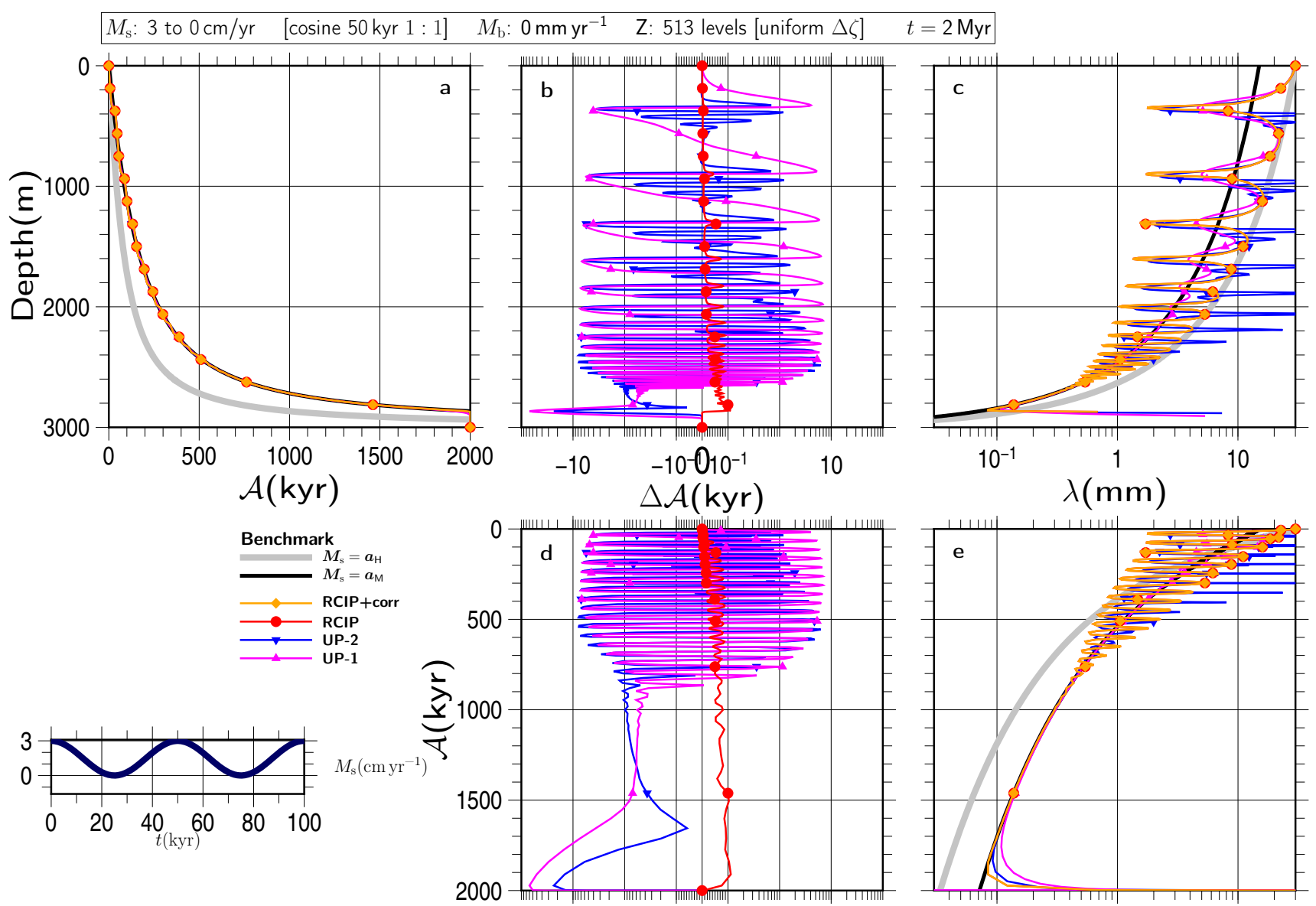

Figure S76 

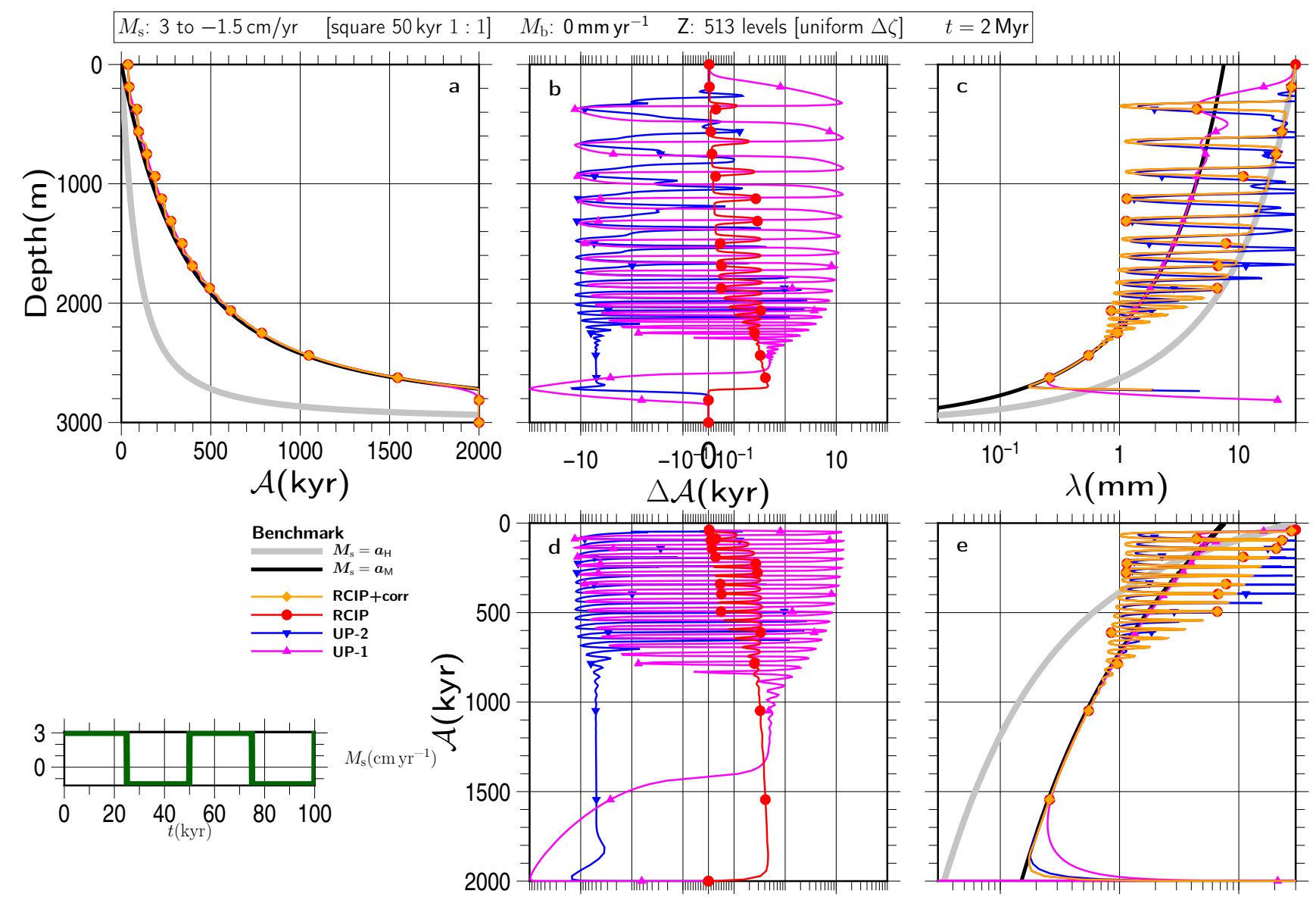

Figure S77
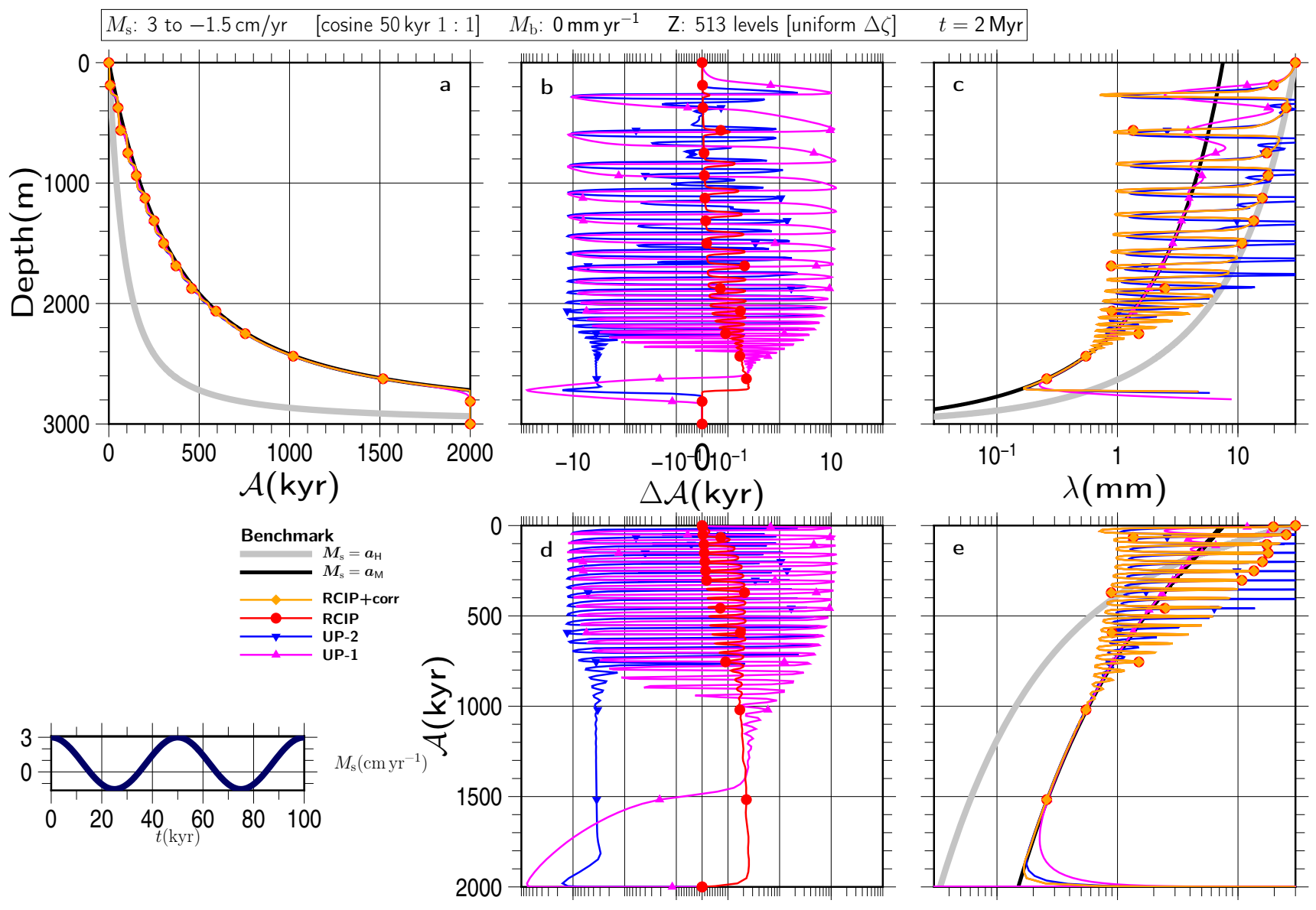

Figure S78 

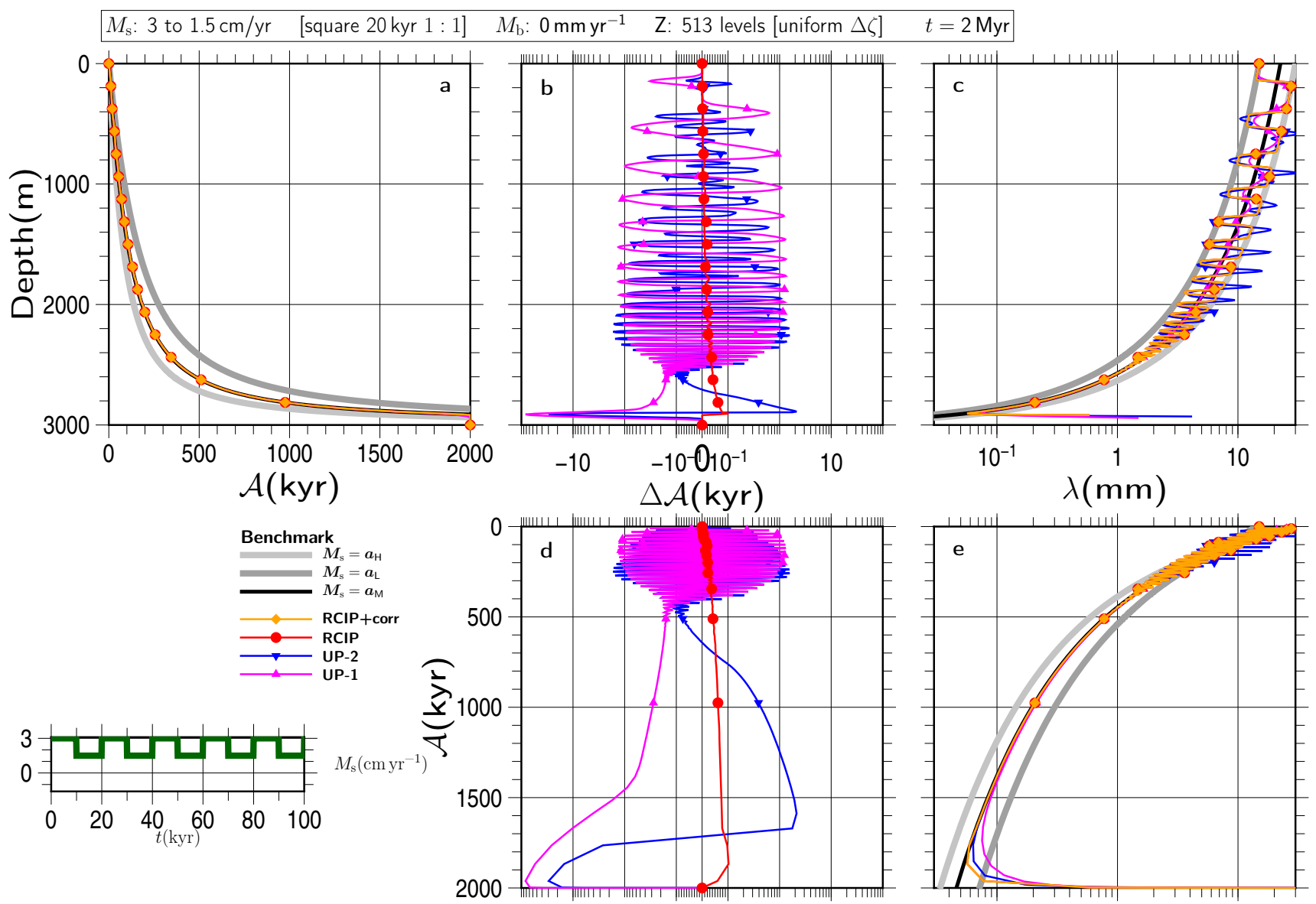

Figure S79
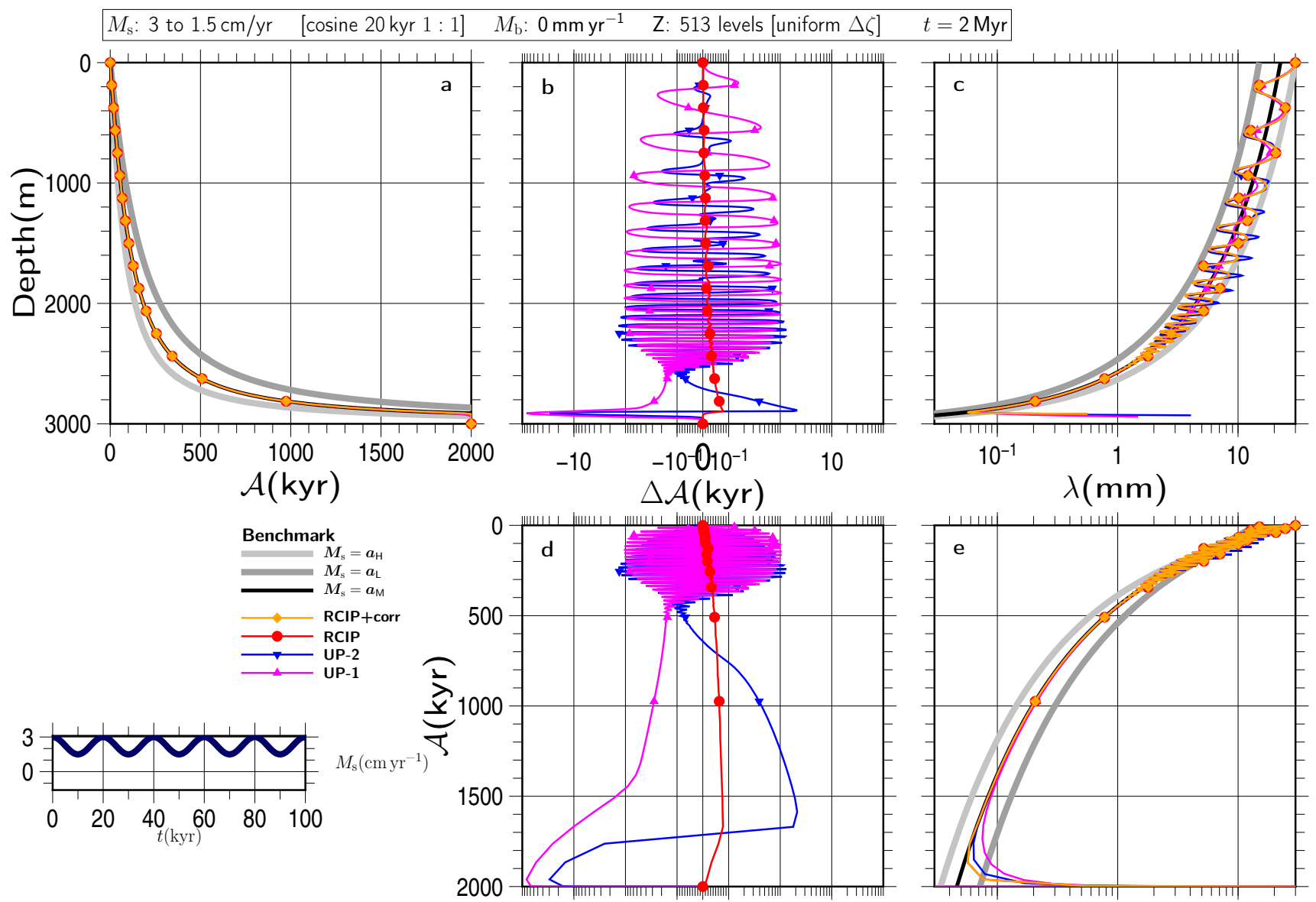

Figure S80 

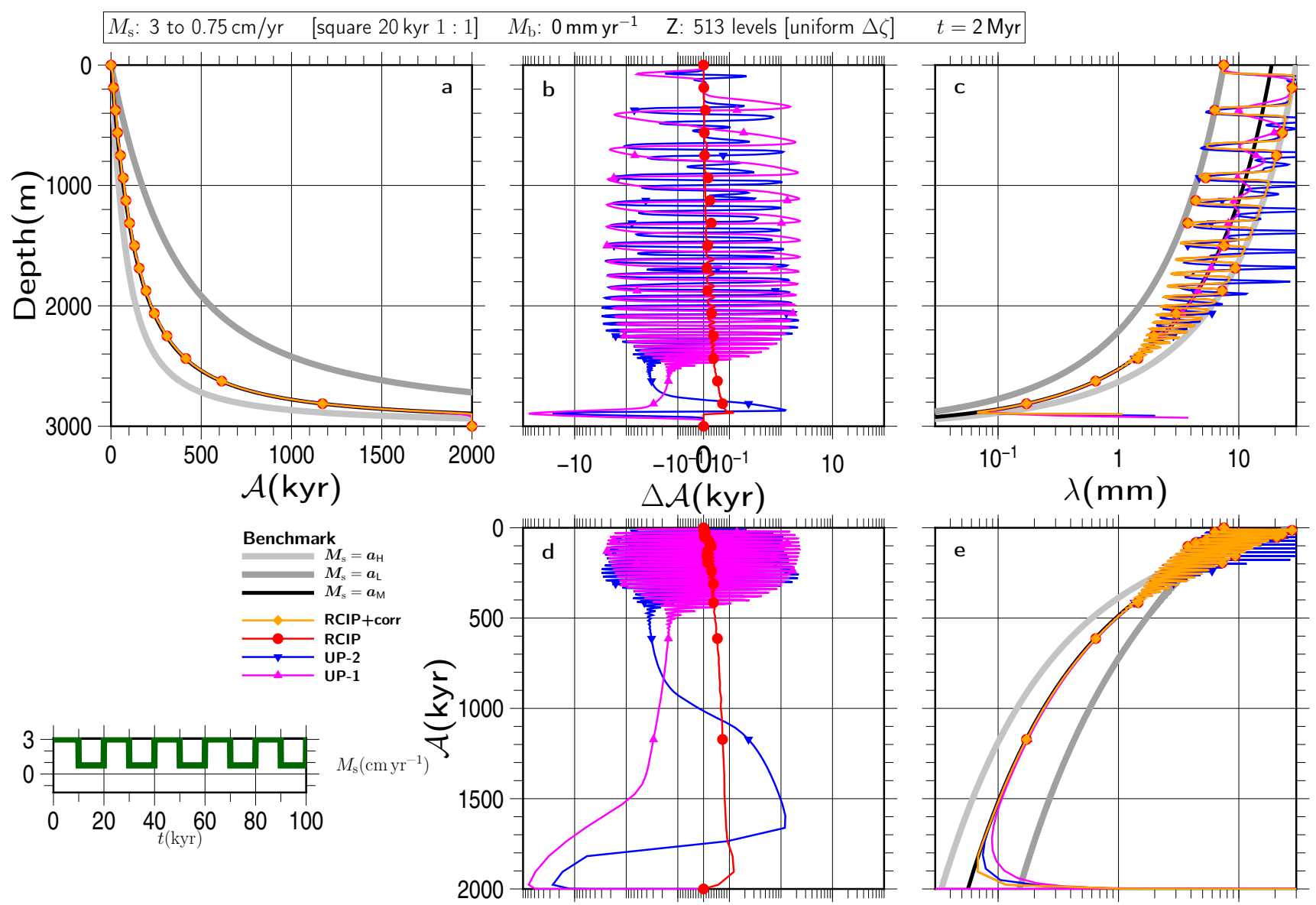

Figure S81
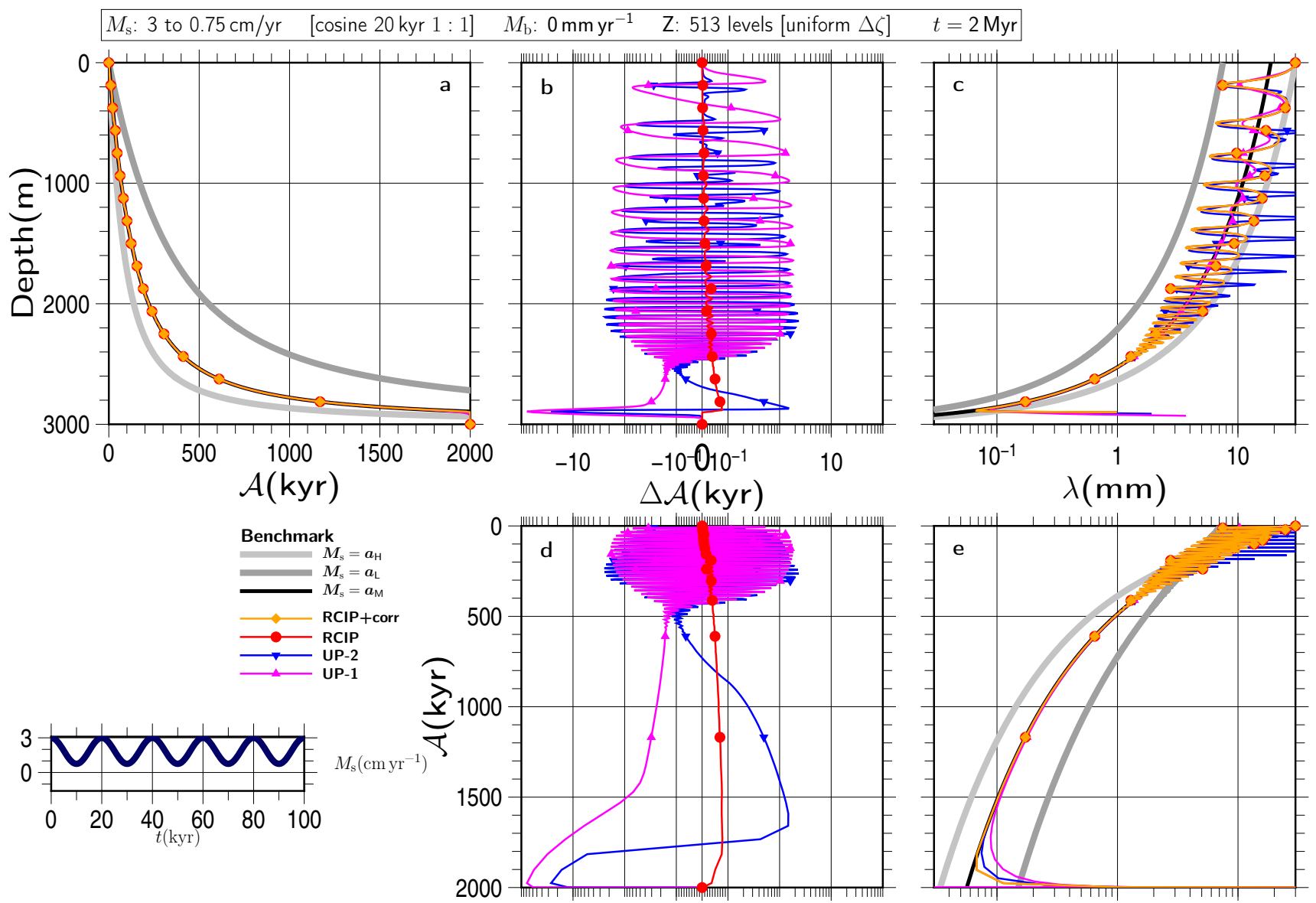

Figure S82 

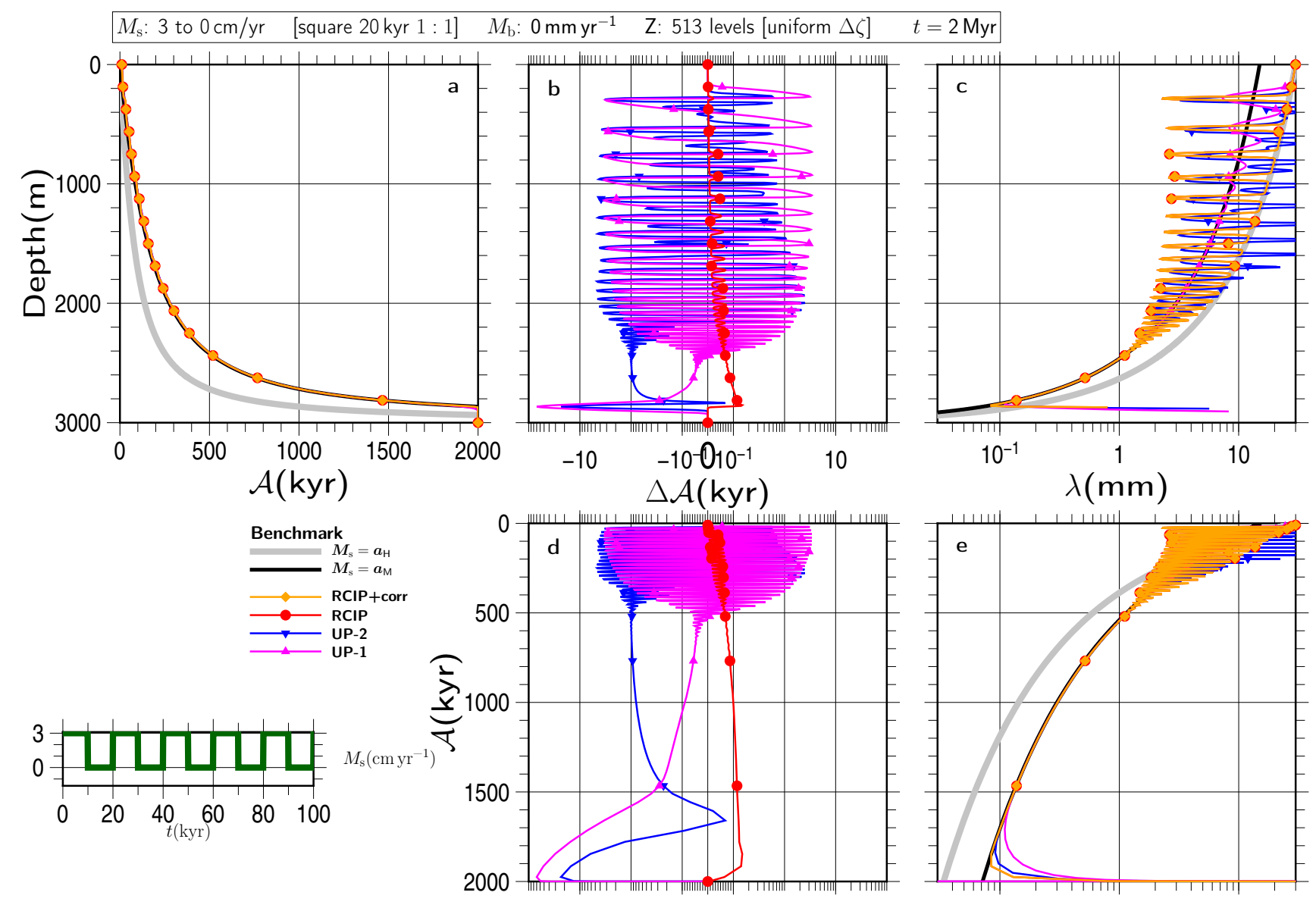

Figure S83
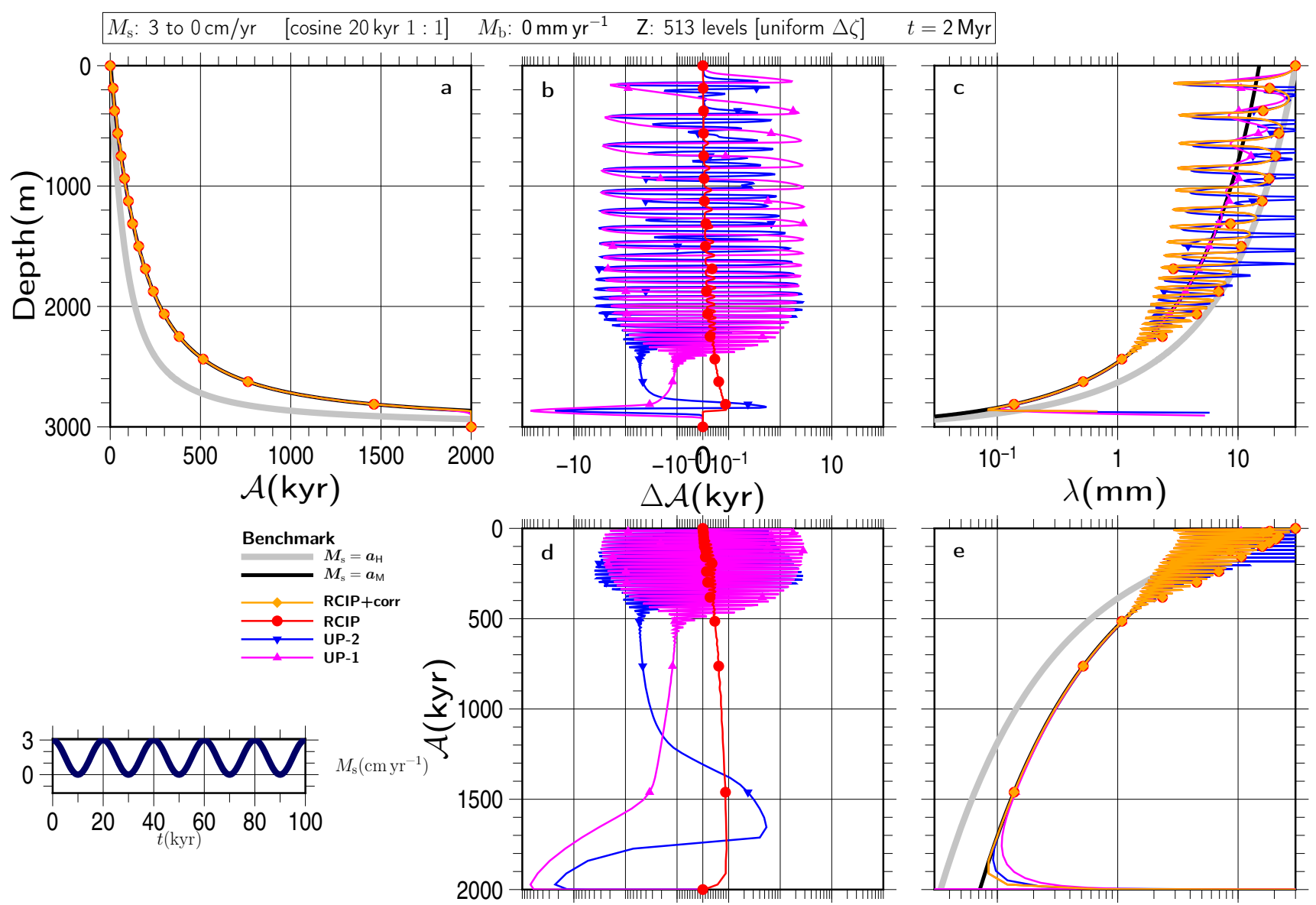

Figure S84 

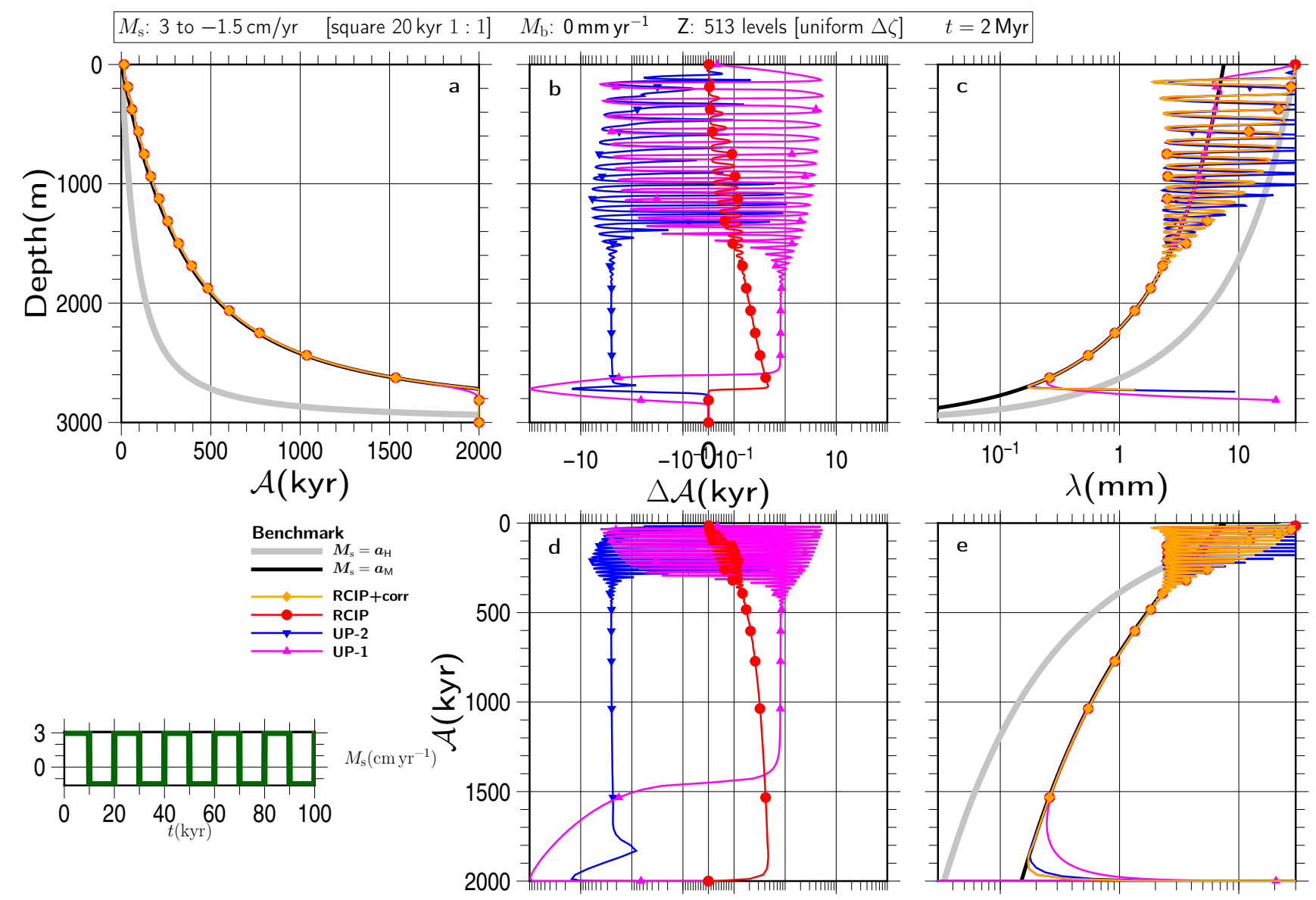

Figure S85
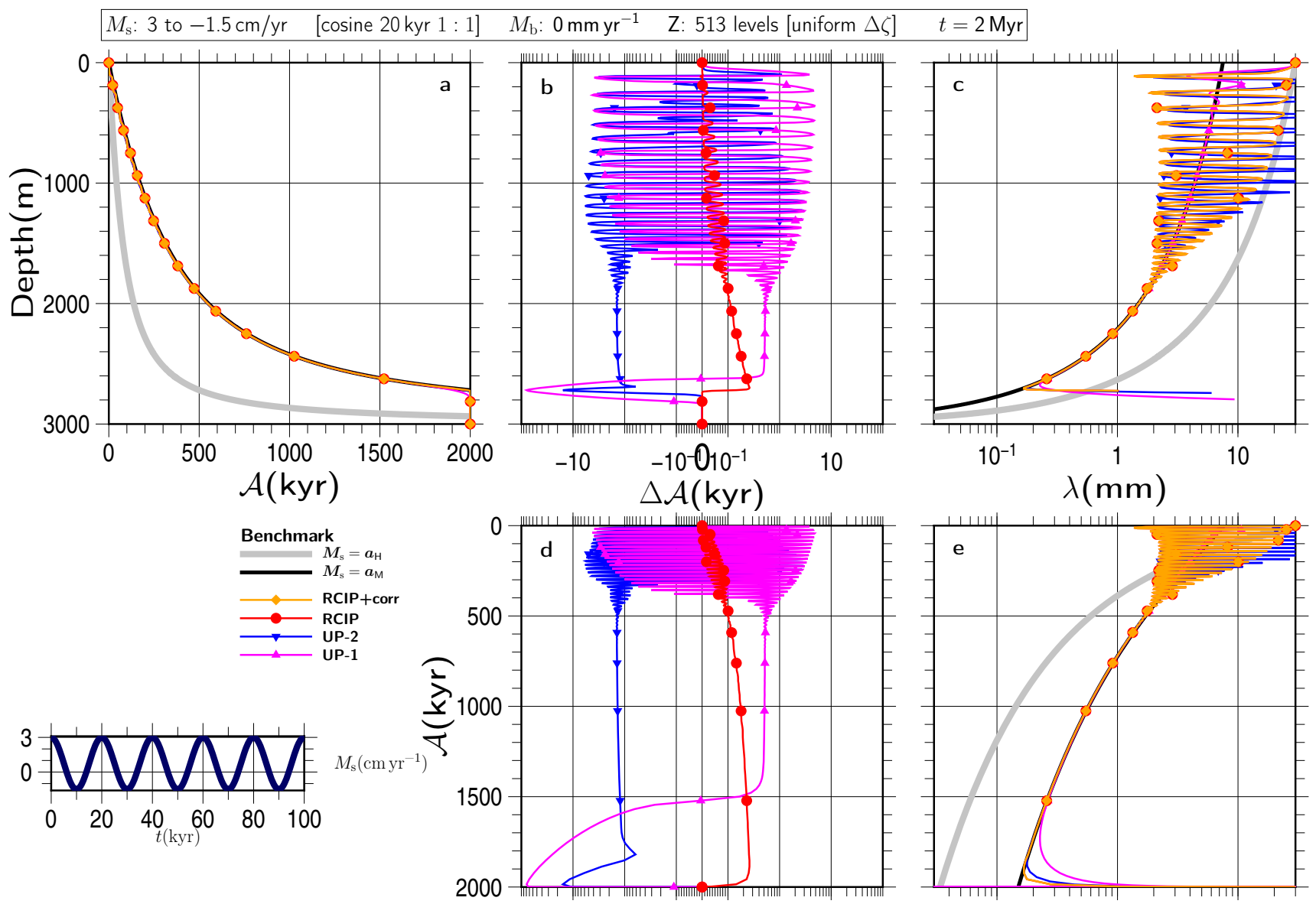

Figure S86 

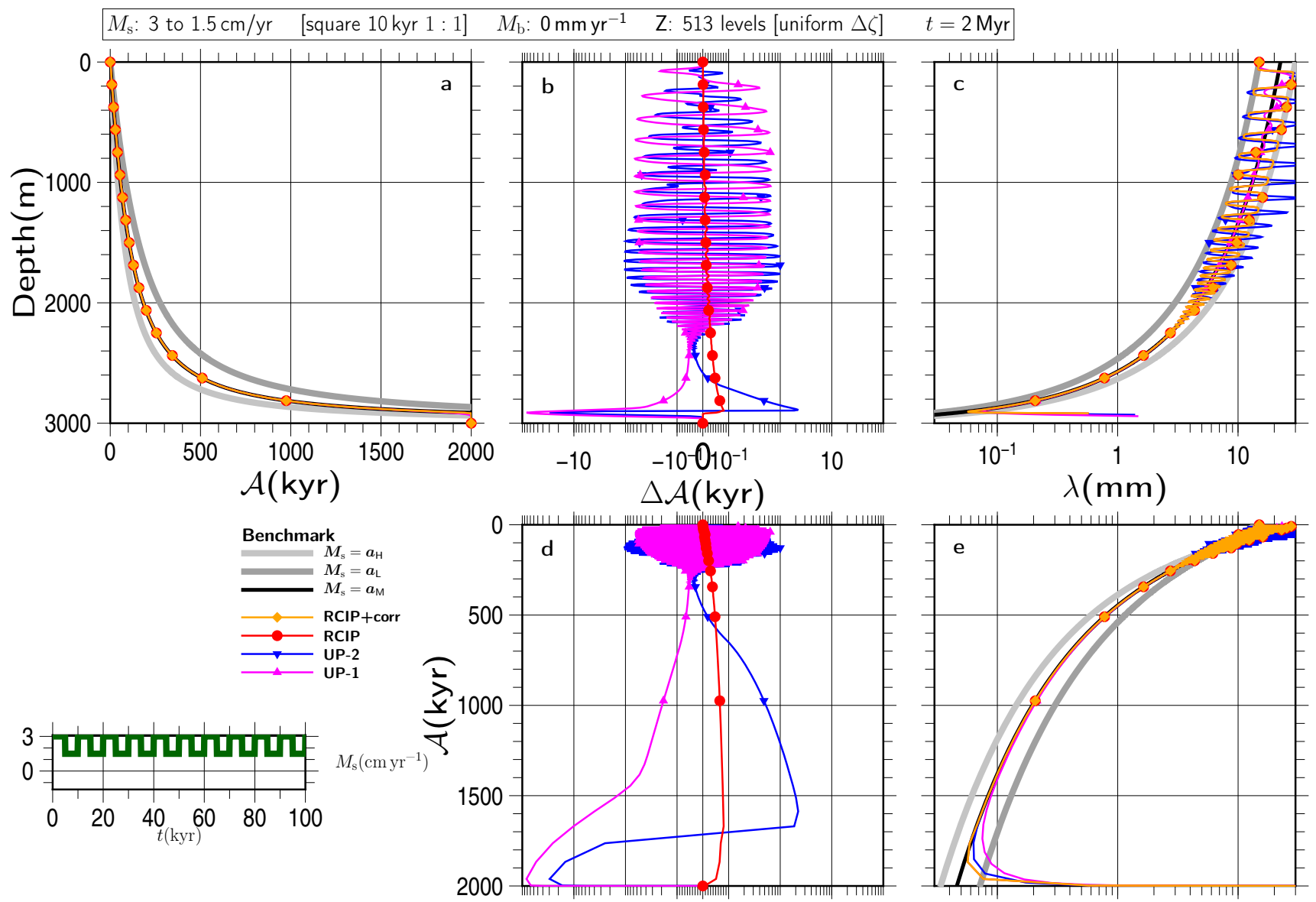

Figure S87
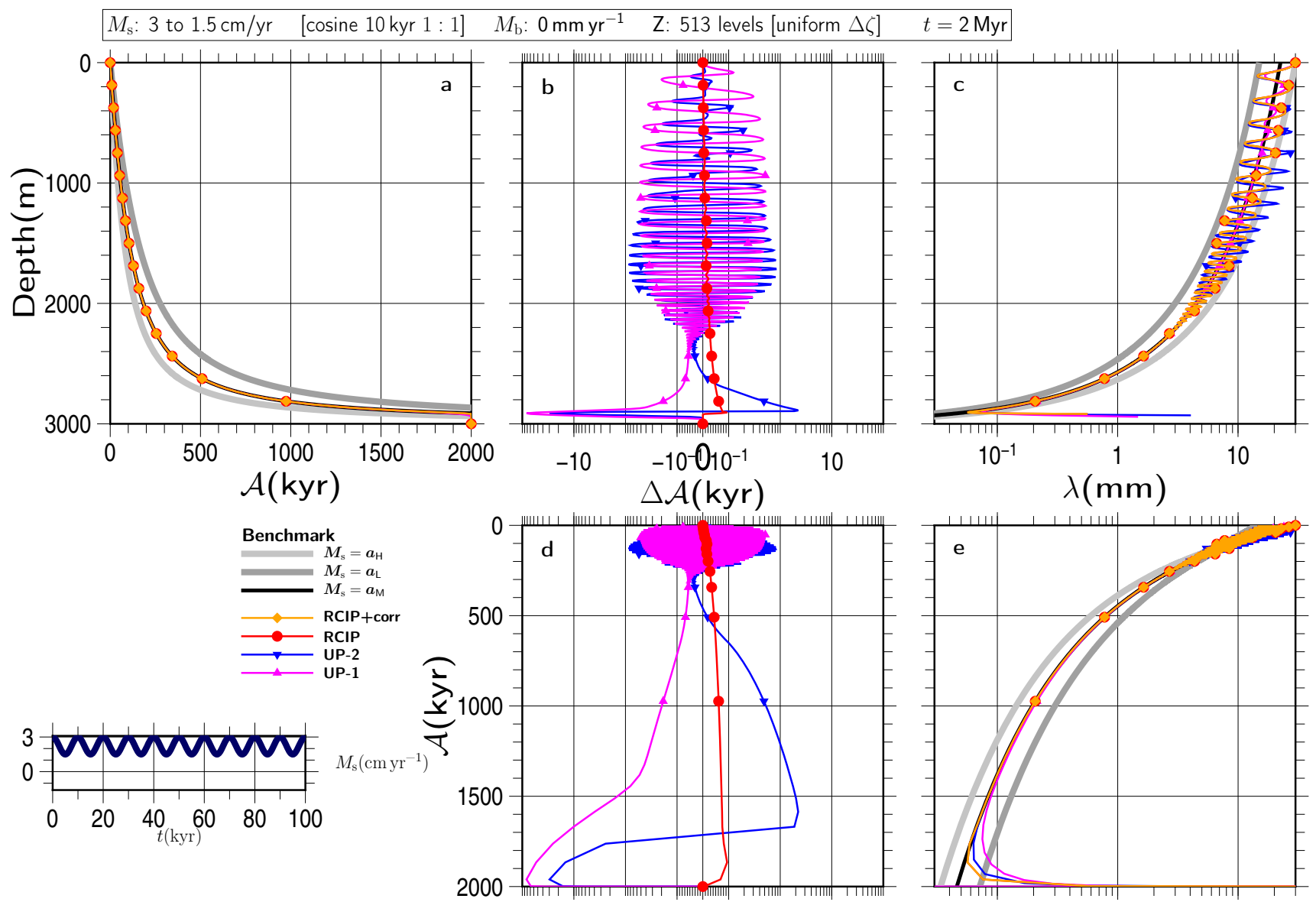

Figure S88 

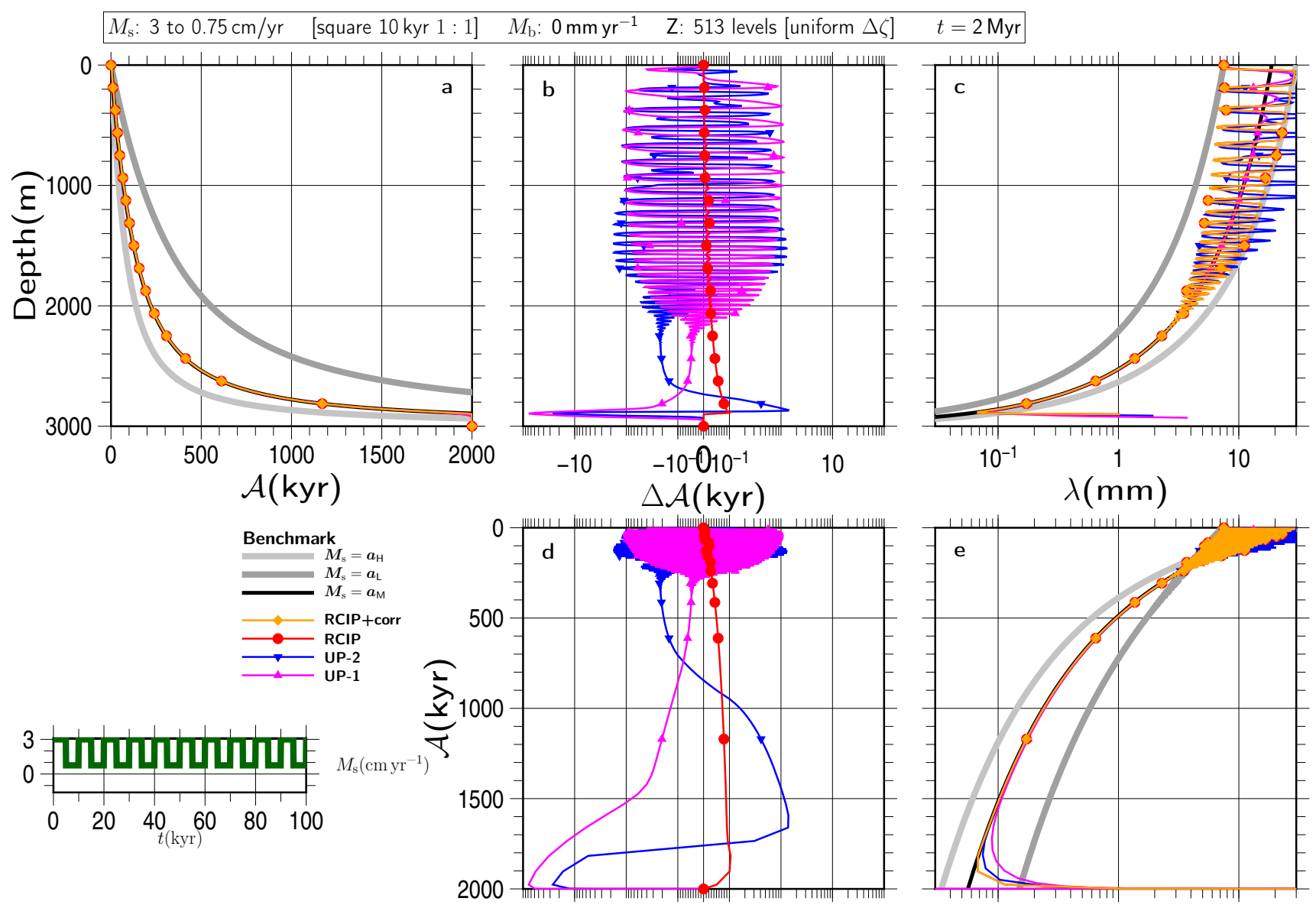

Figure S89
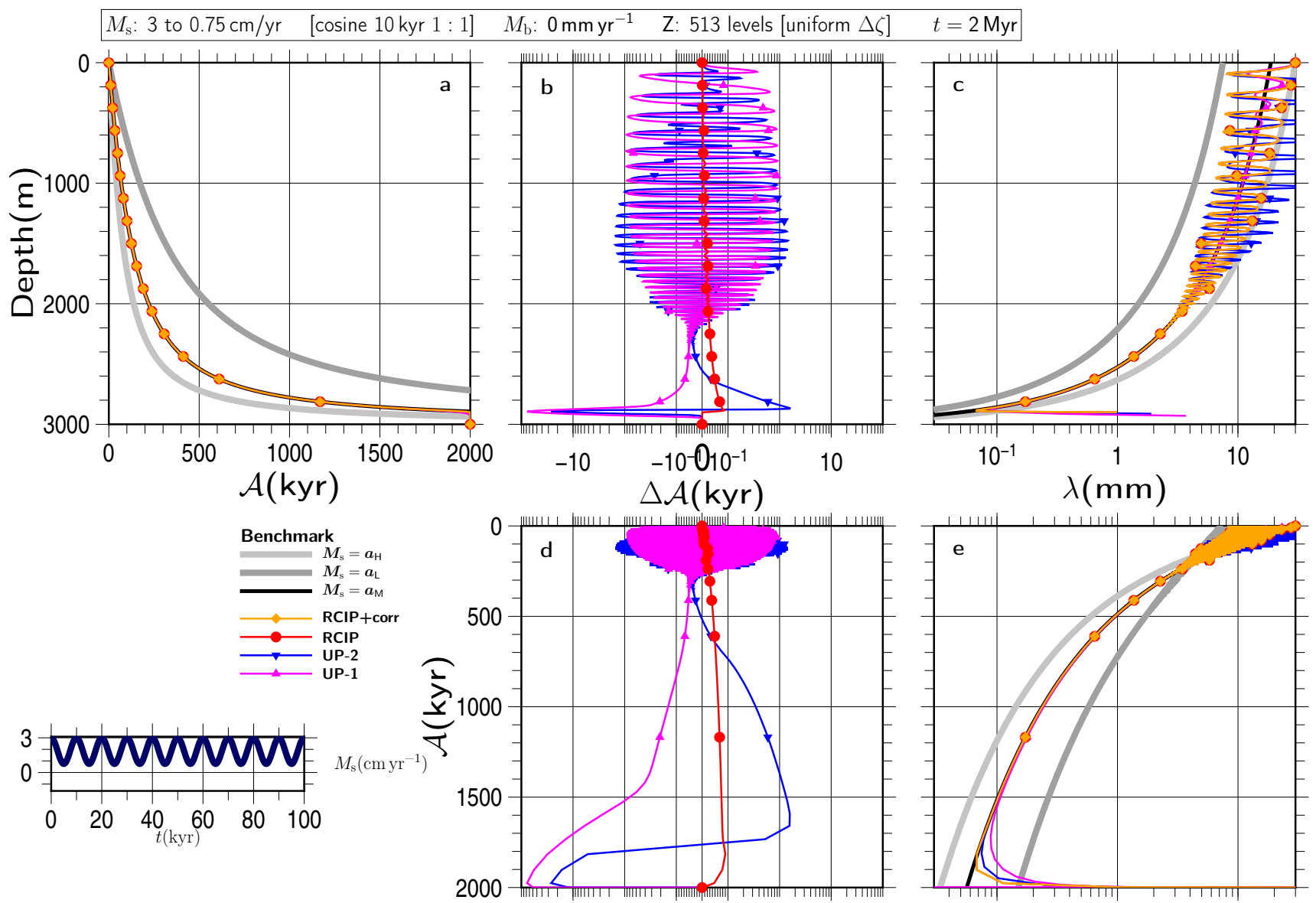

Figure S90 

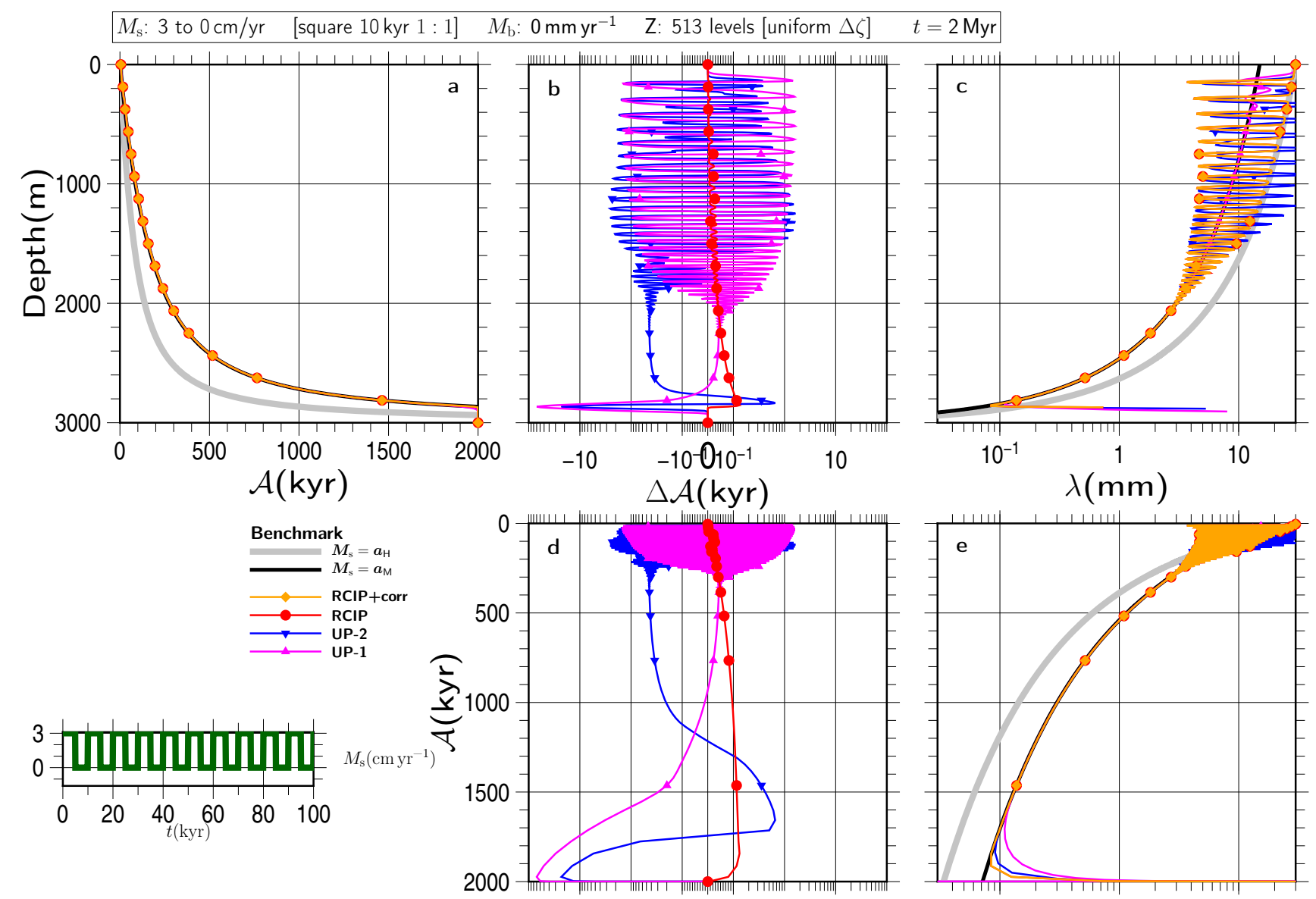

Figure S91
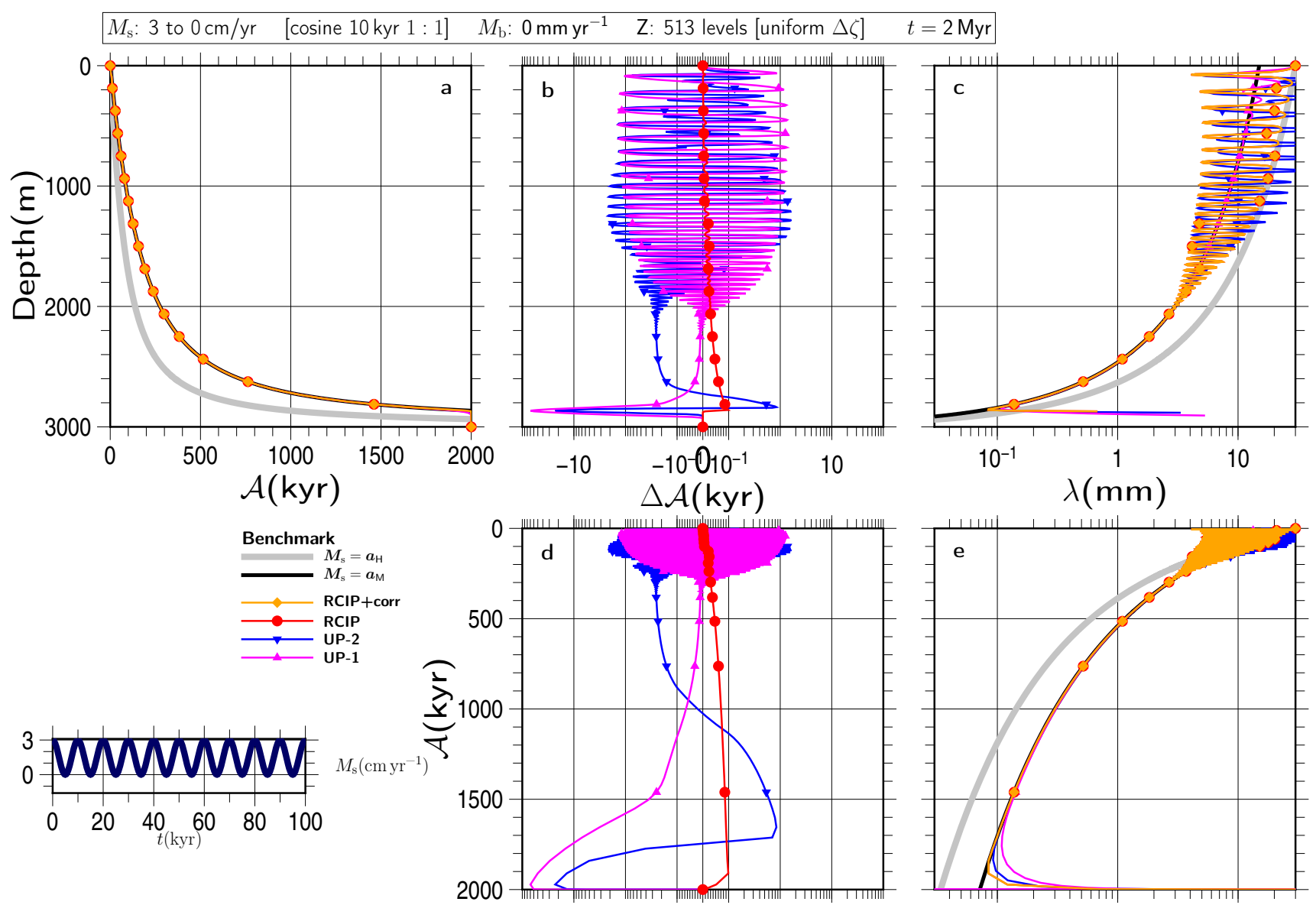

Figure S92 

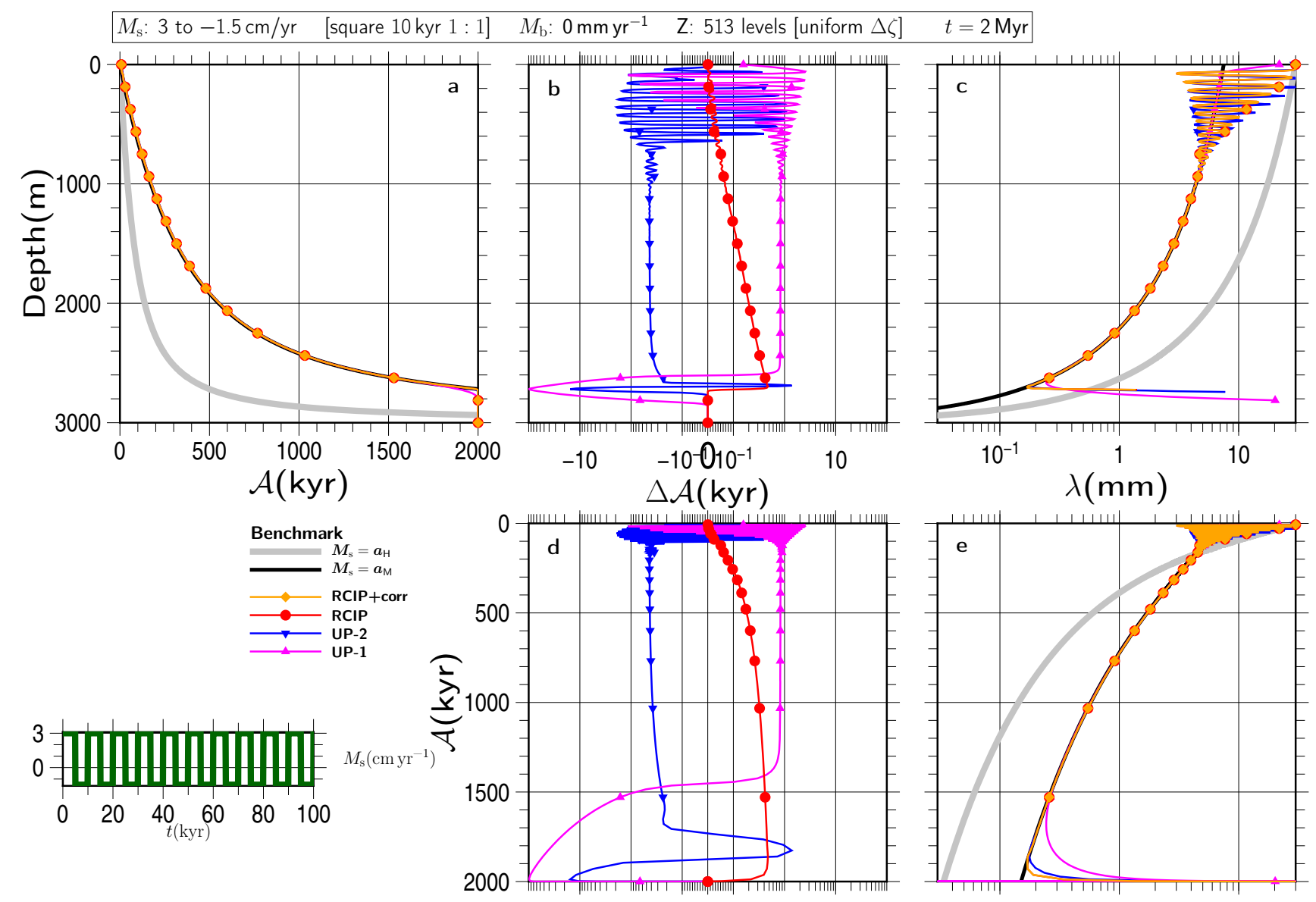

Figure S93
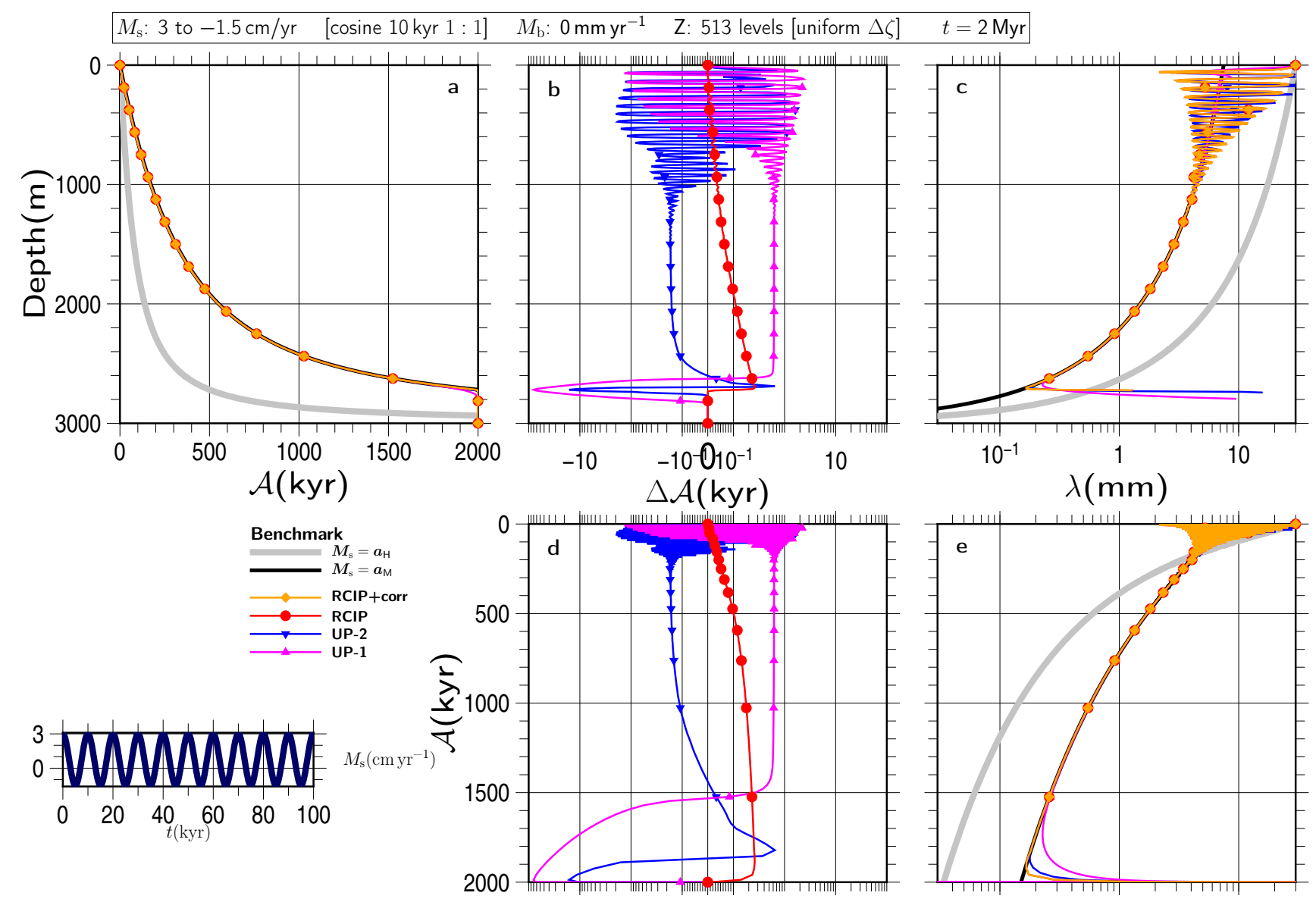

Figure S94 

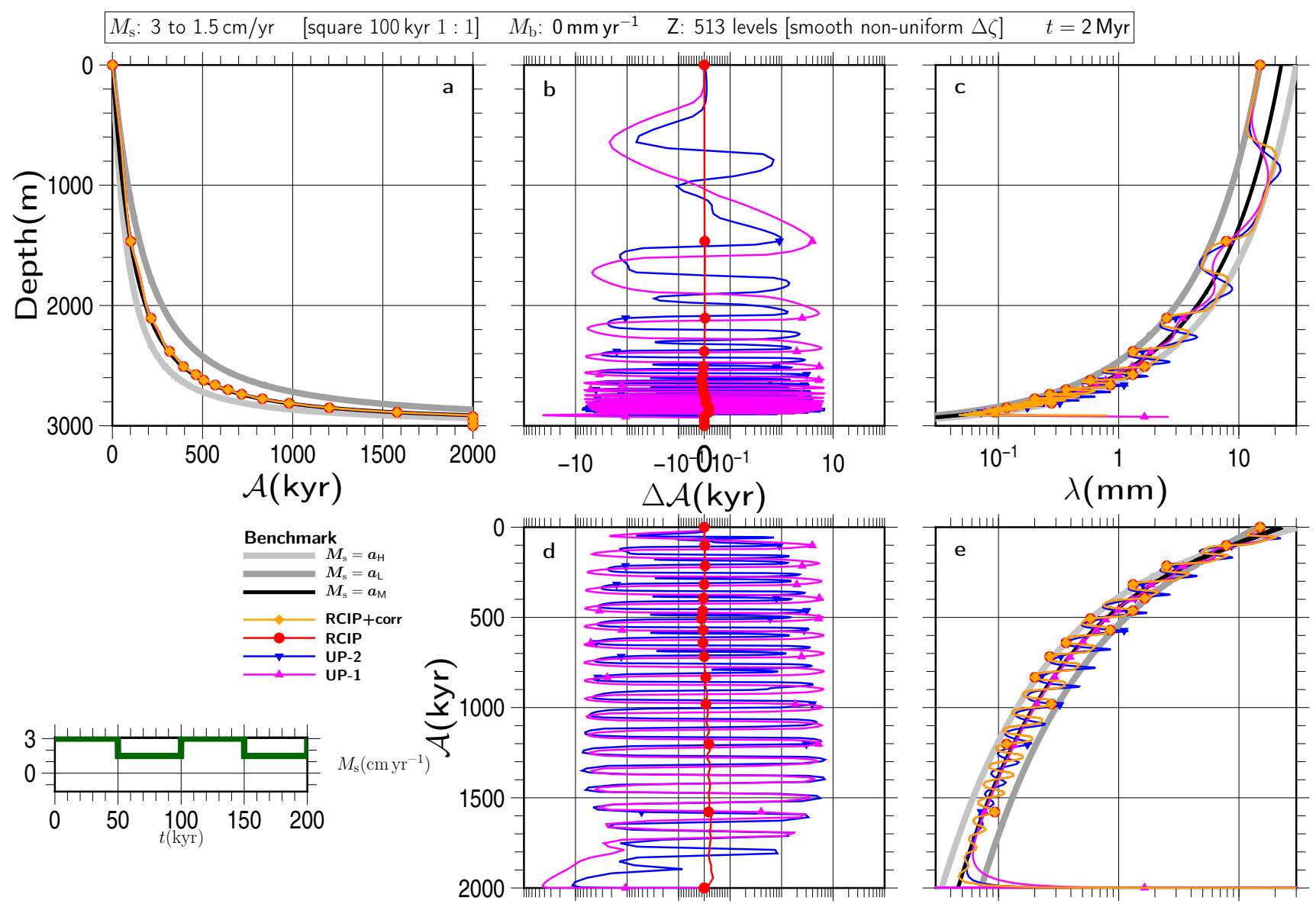

Figure S95
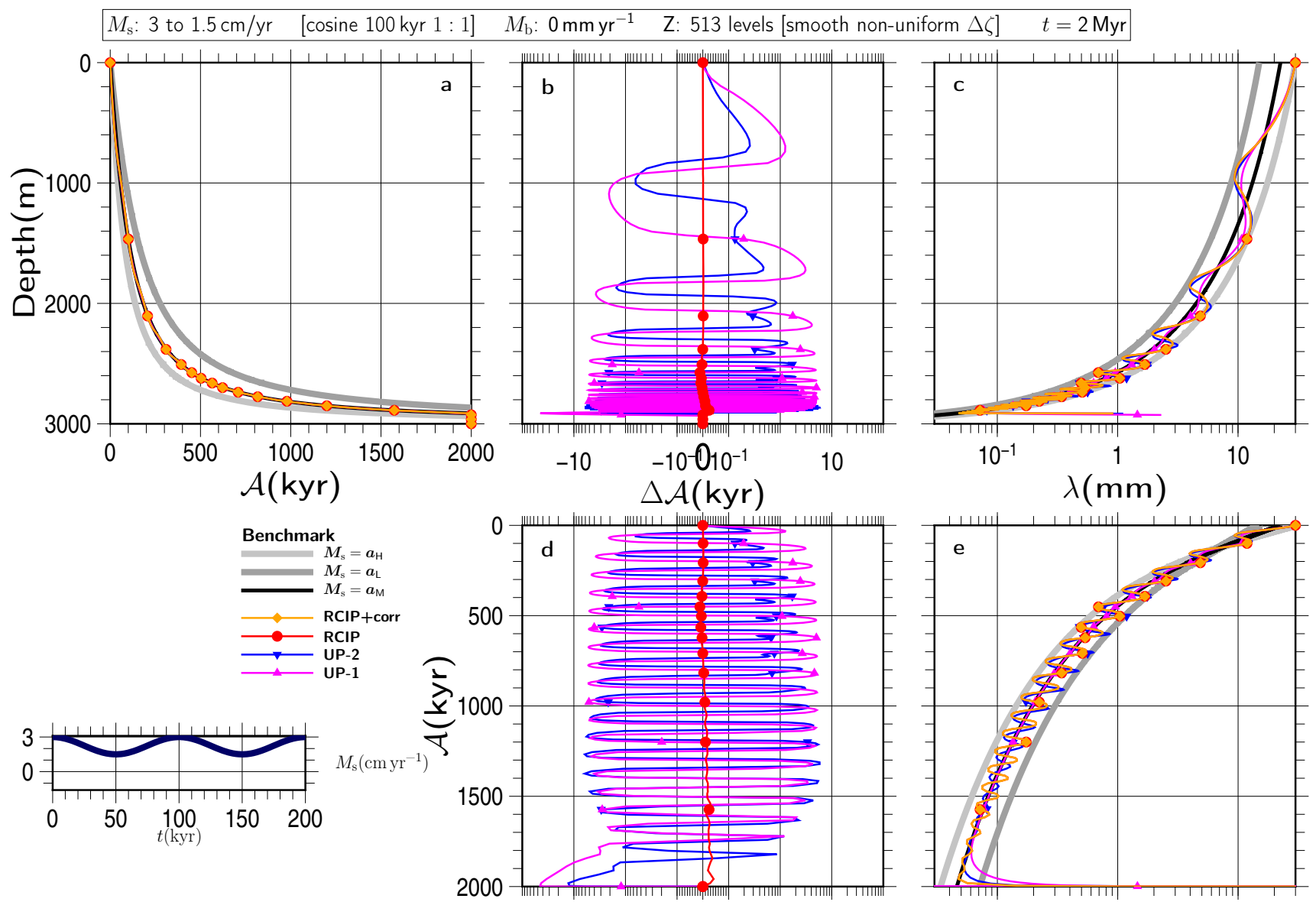

Figure S96 

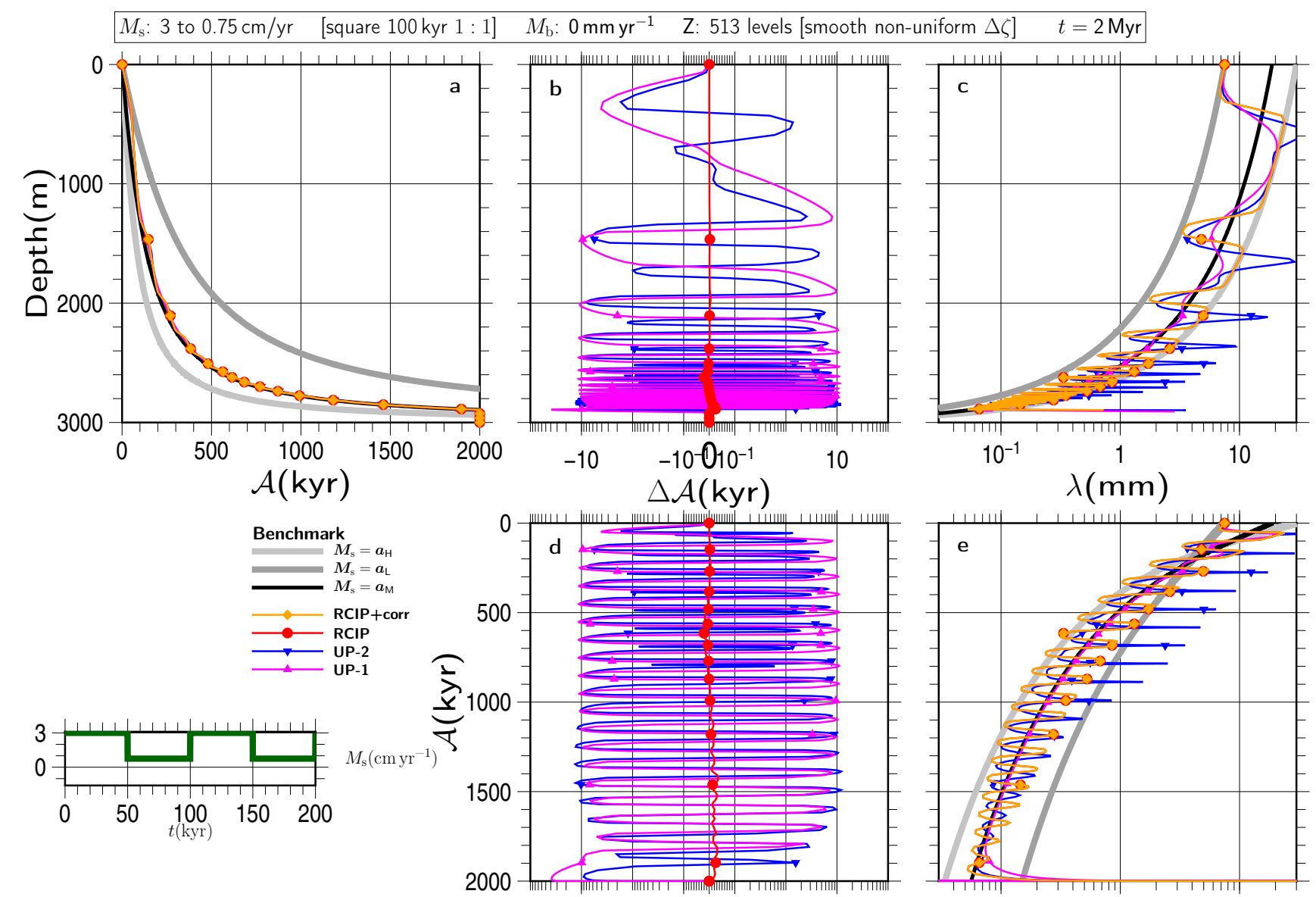

Figure S97
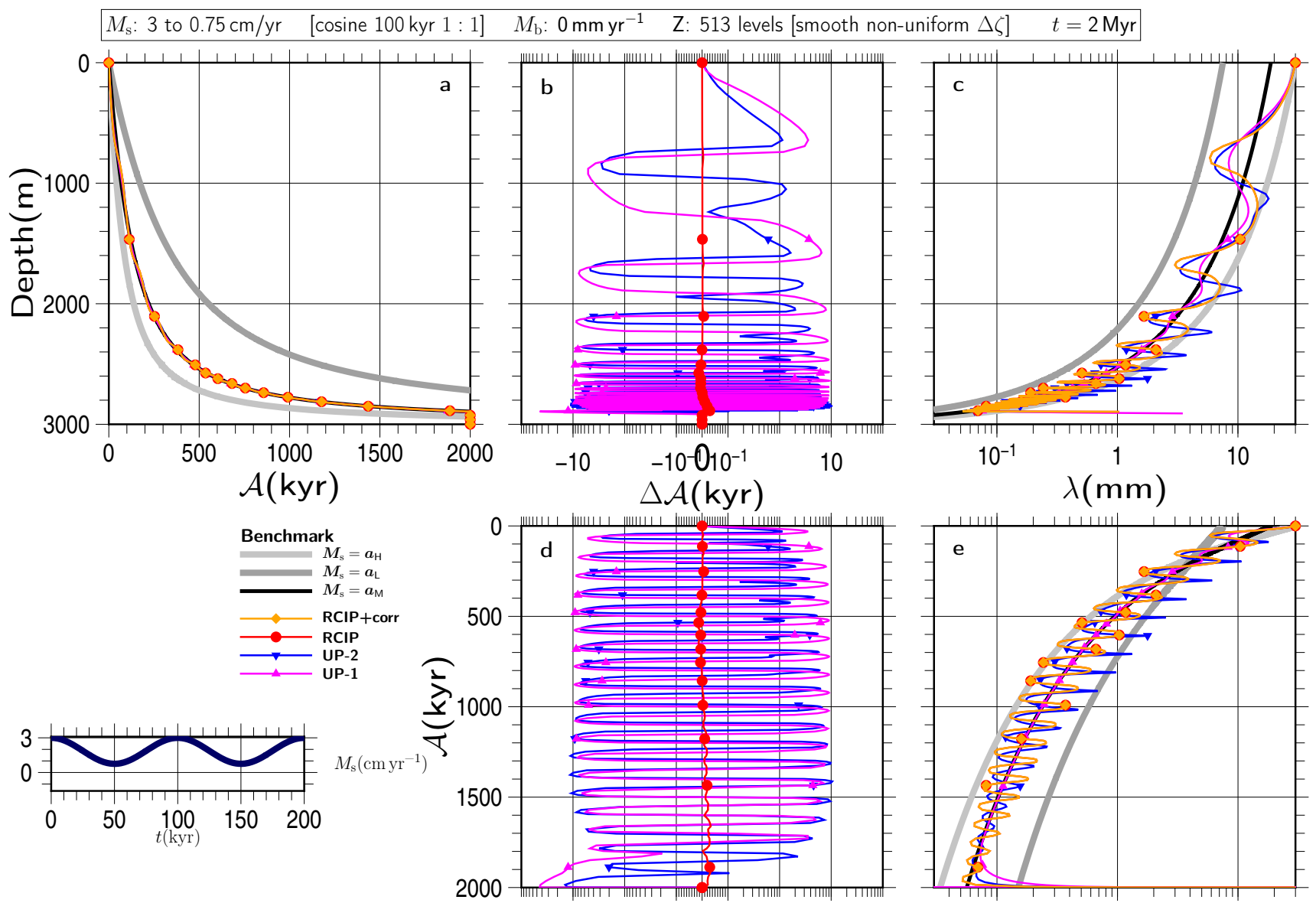

Figure S98 

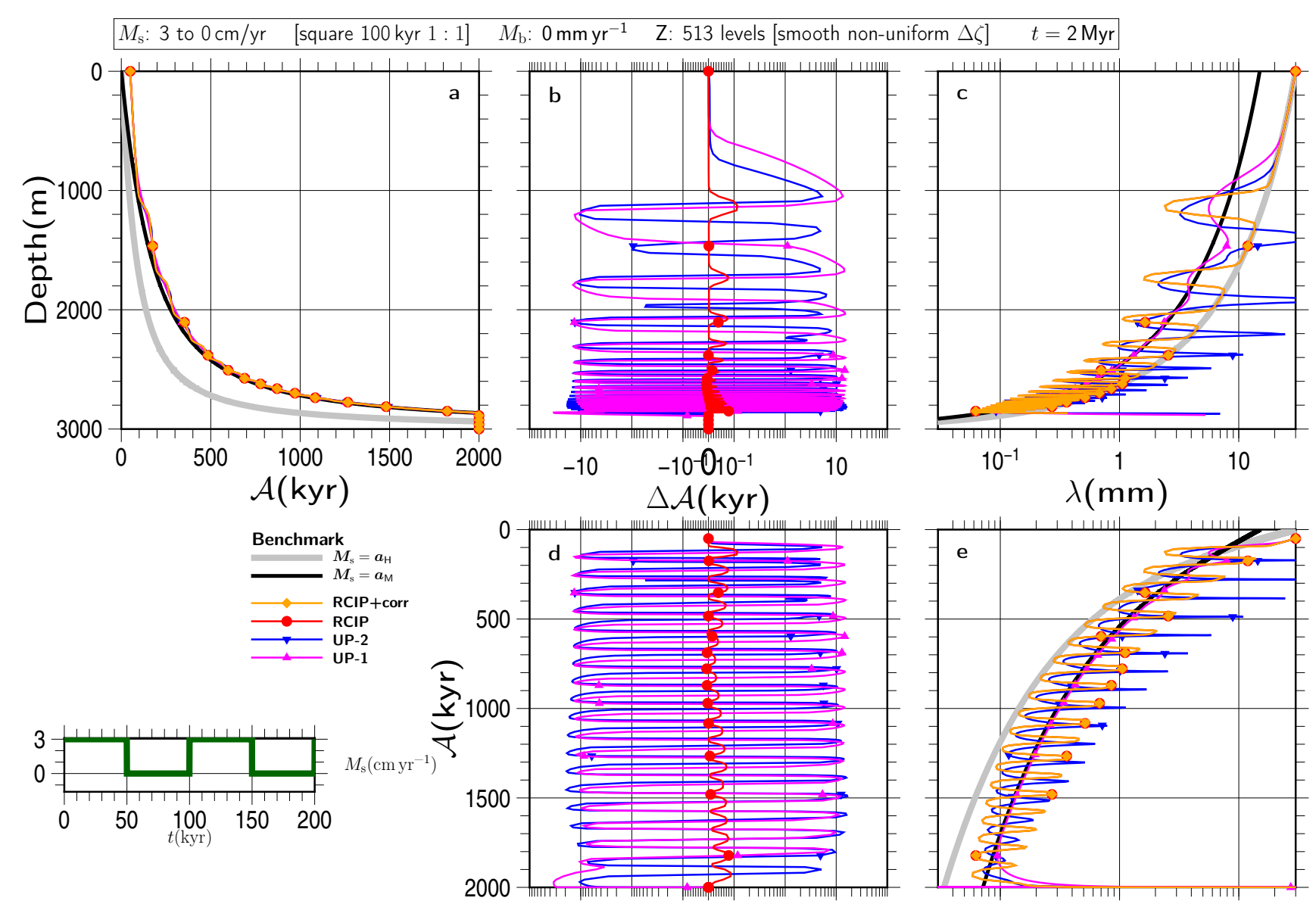

Figure S99
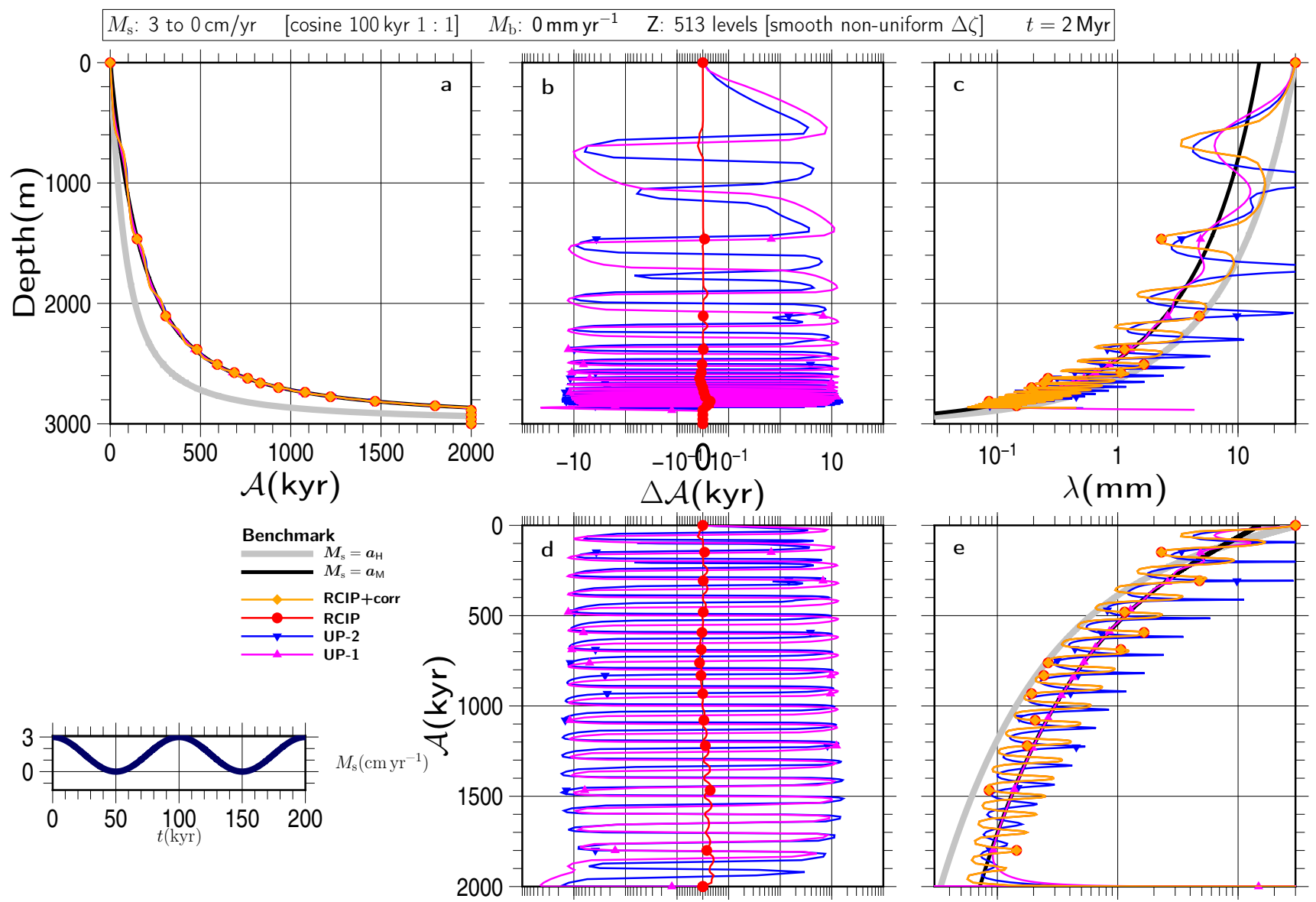

Figure S100 

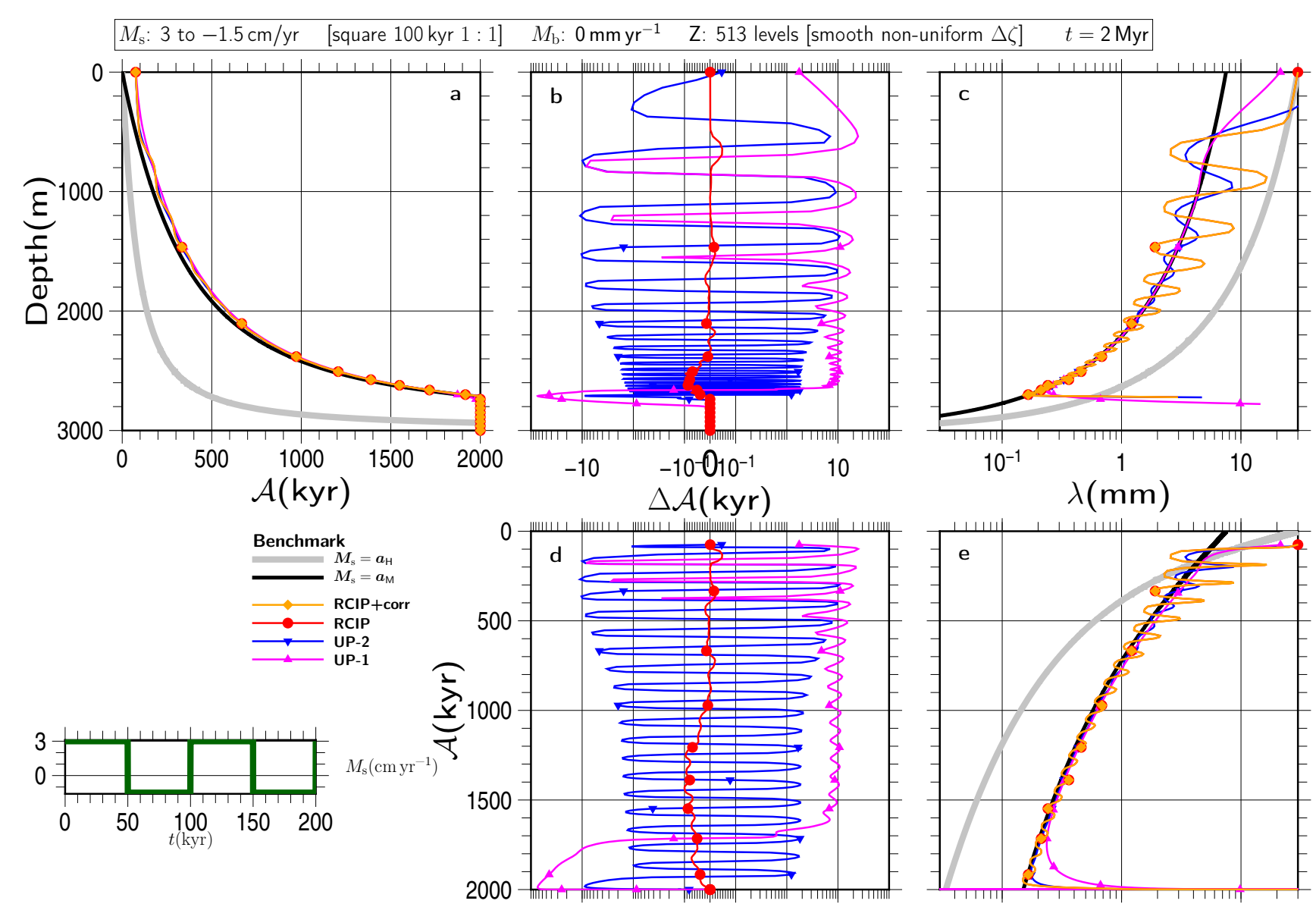

Figure S101
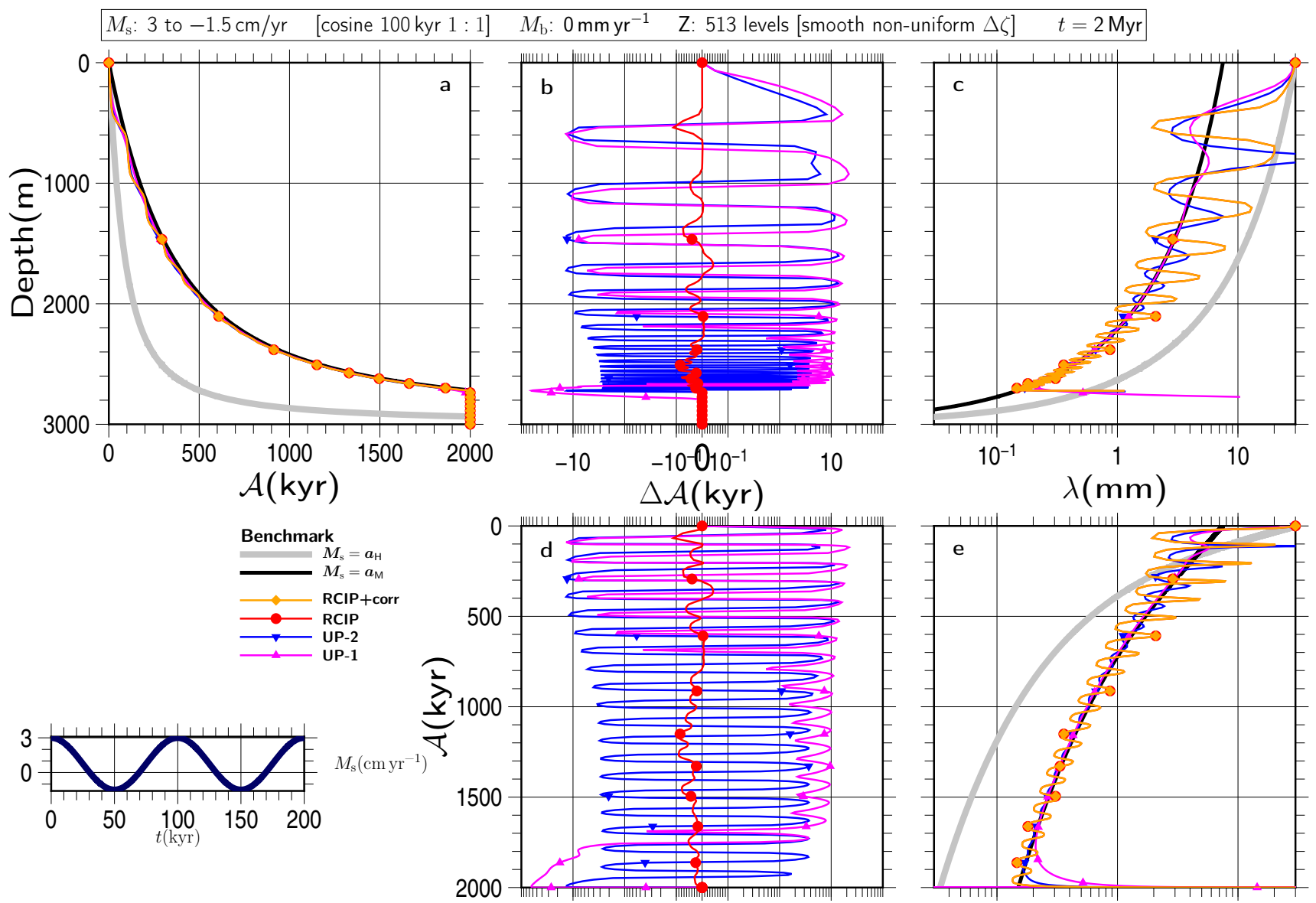

Figure S102 

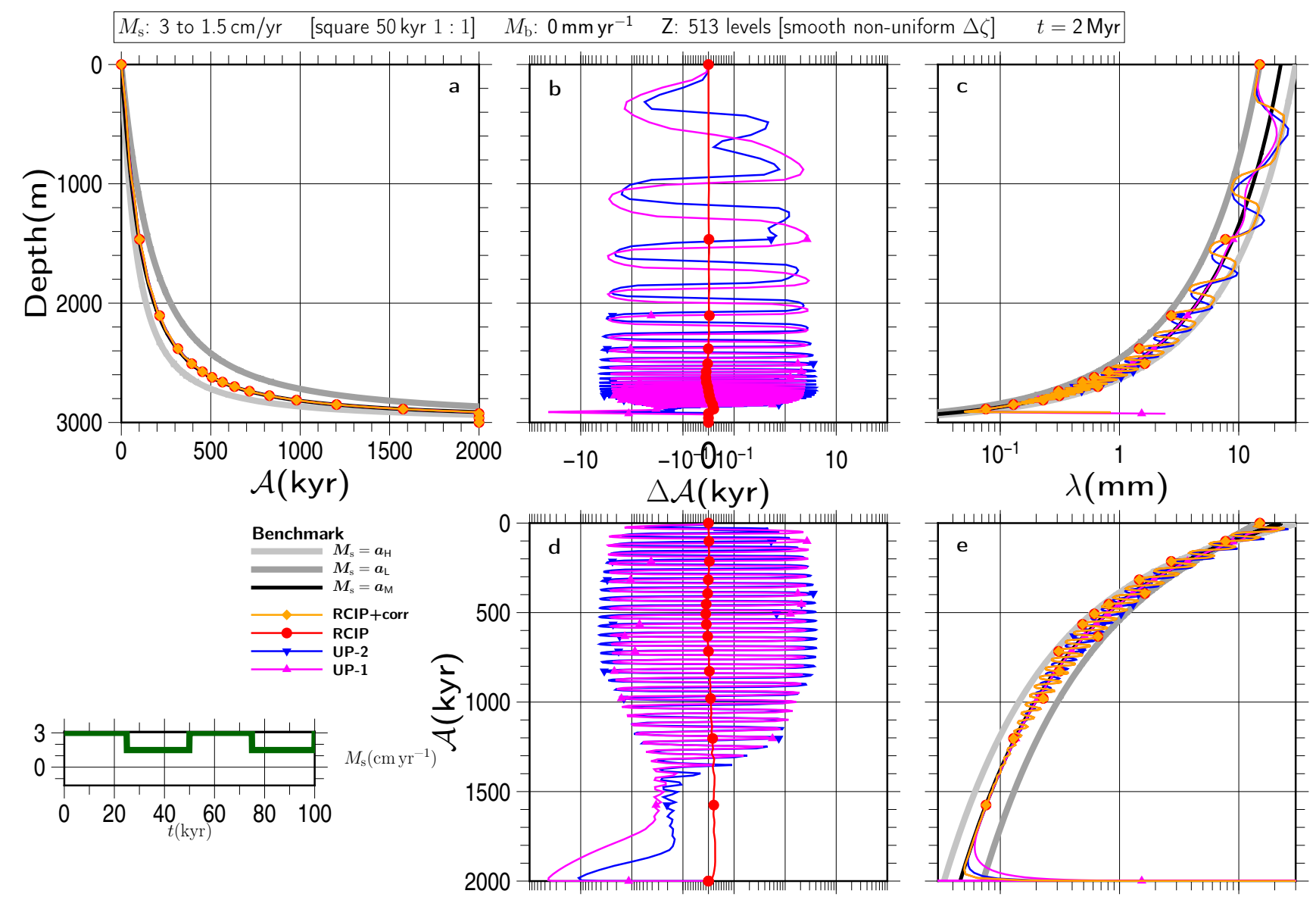

Figure S103
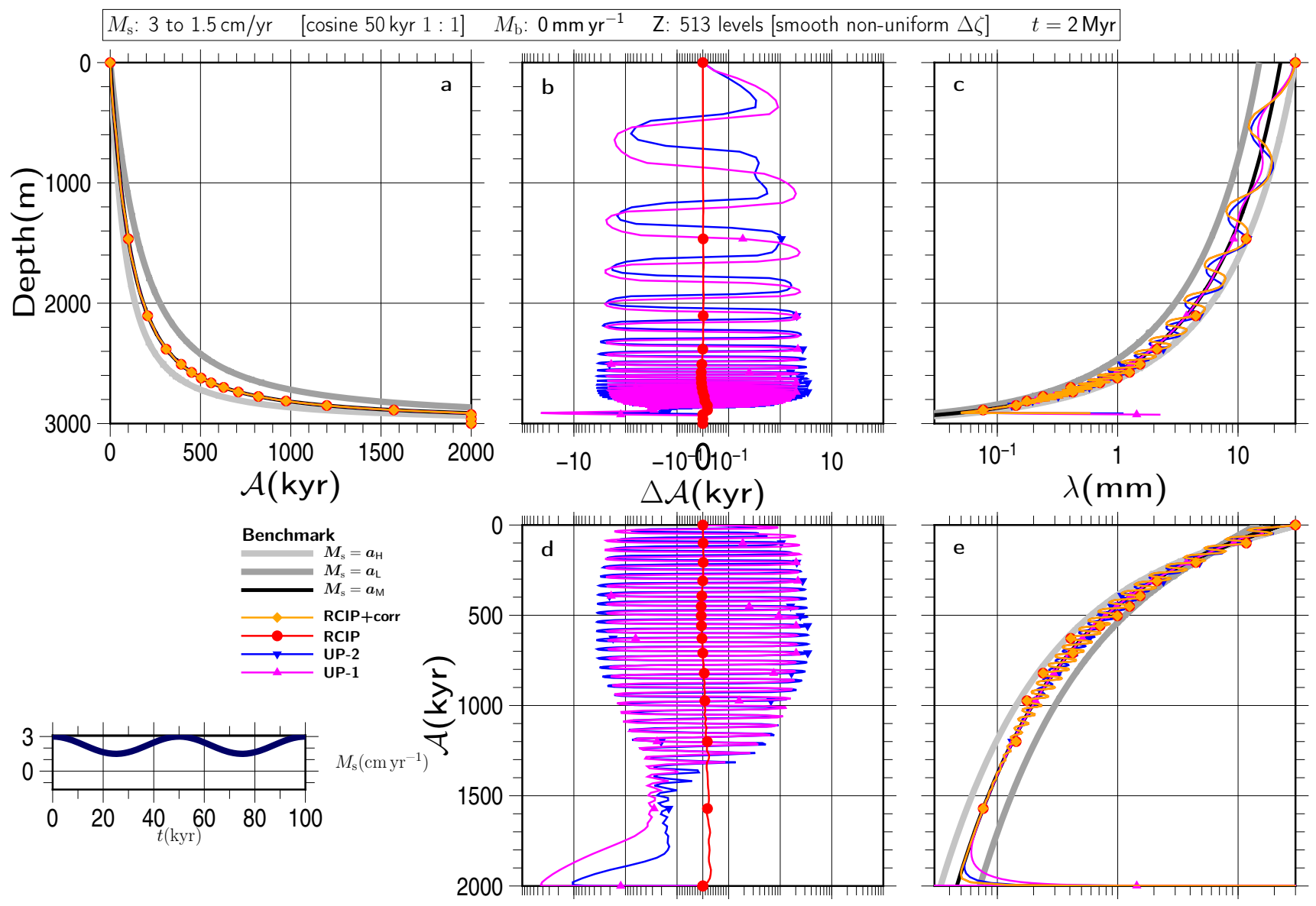

Figure S104 

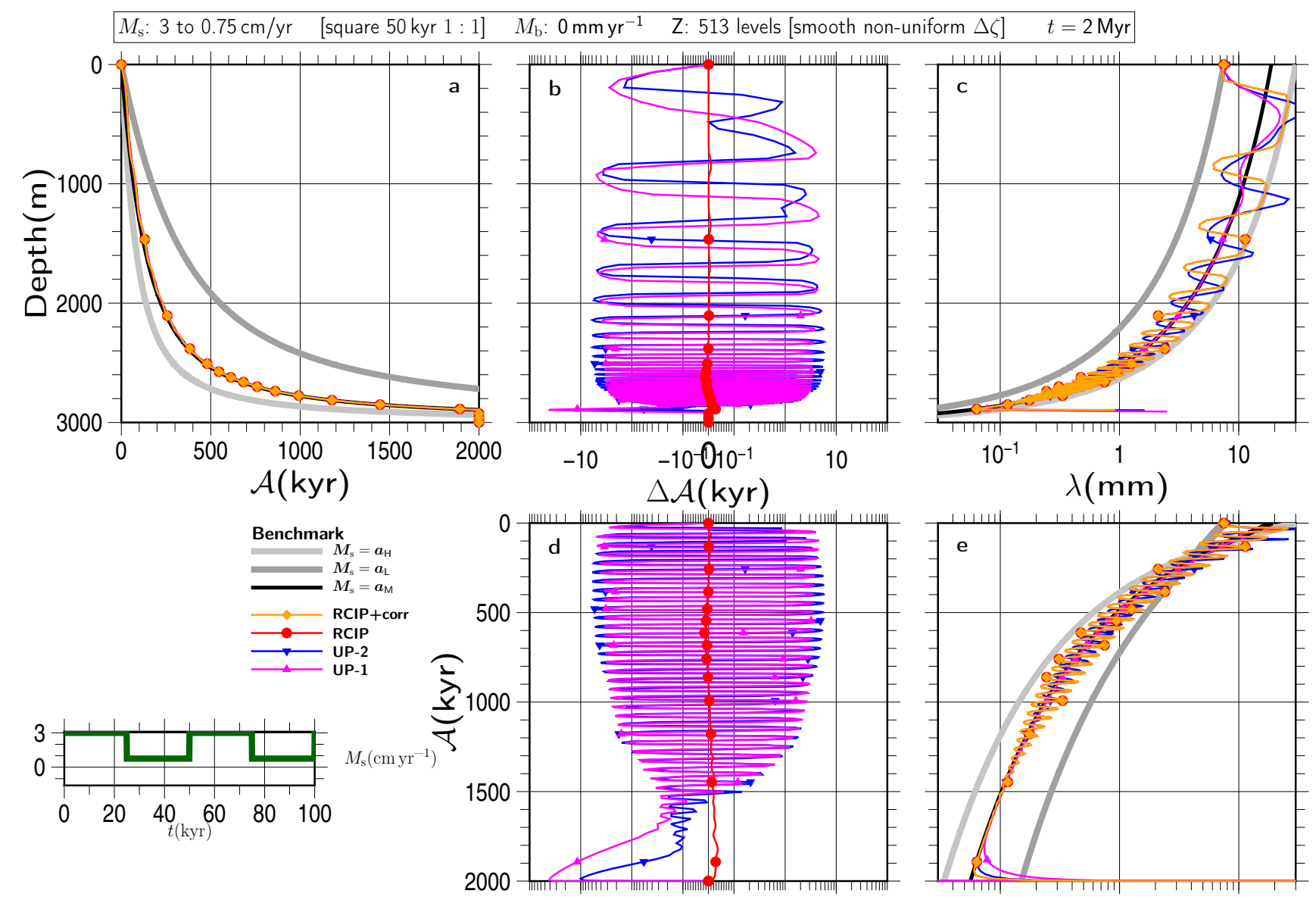

Figure S105
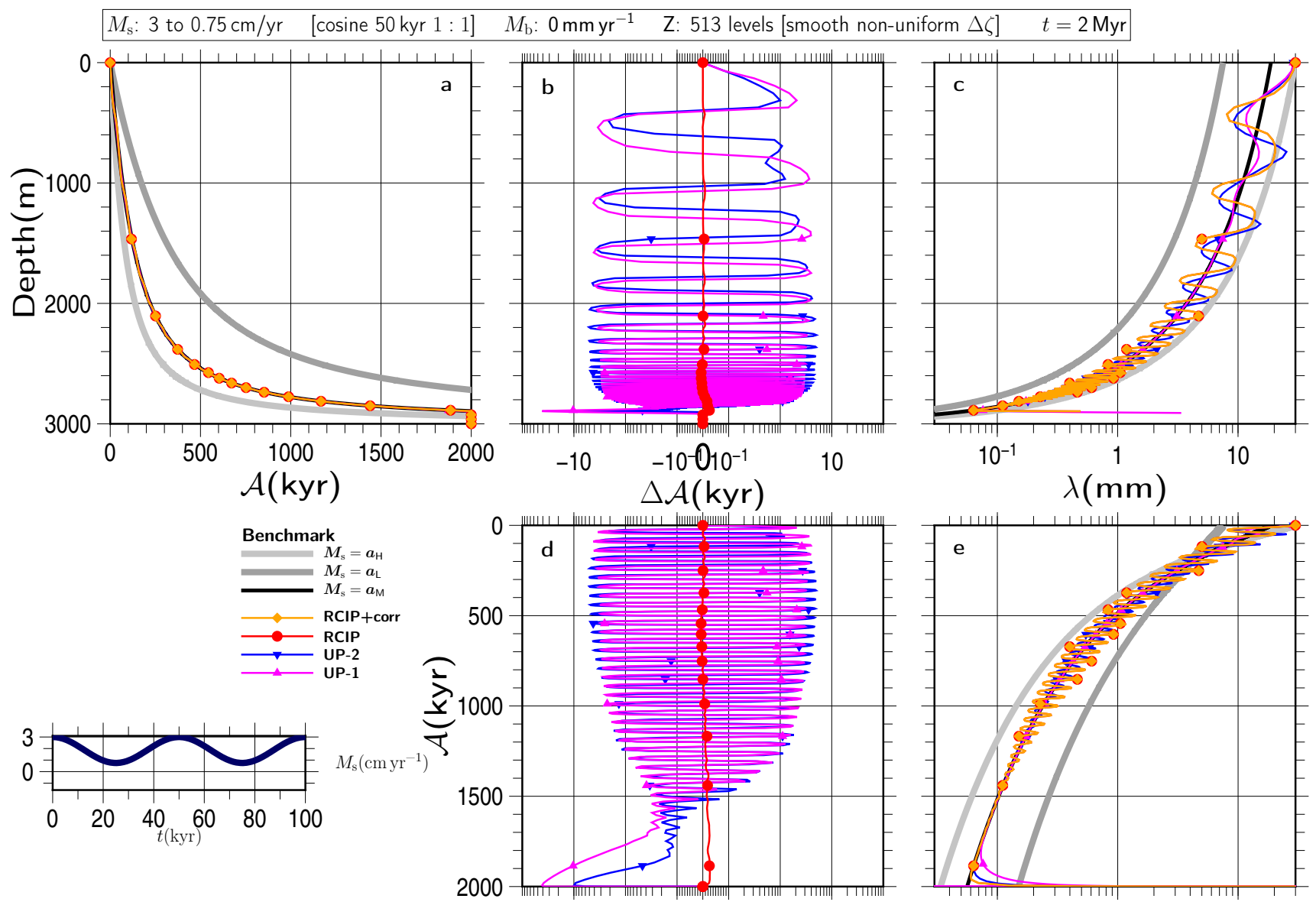

Figure S106 

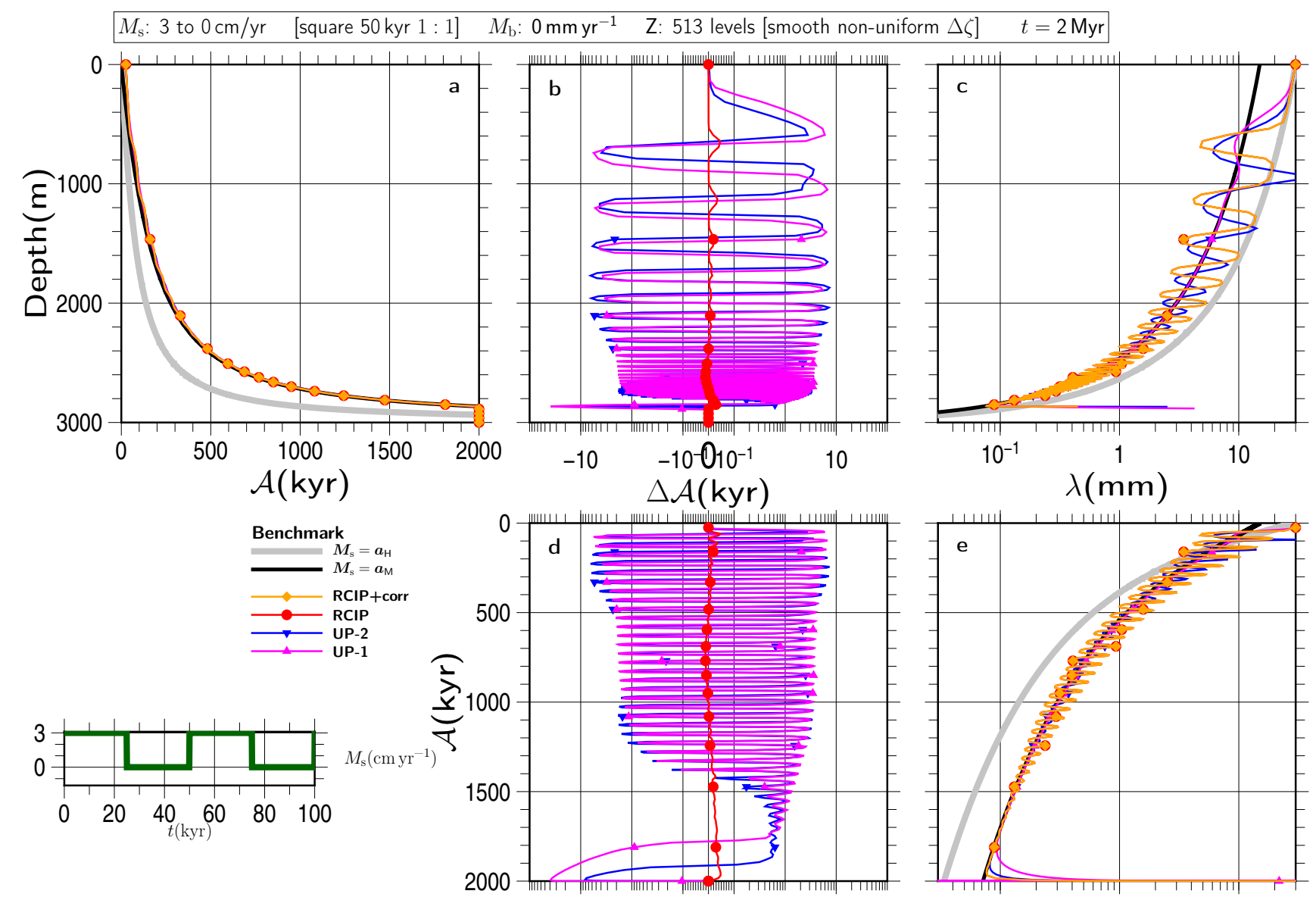

Figure S107
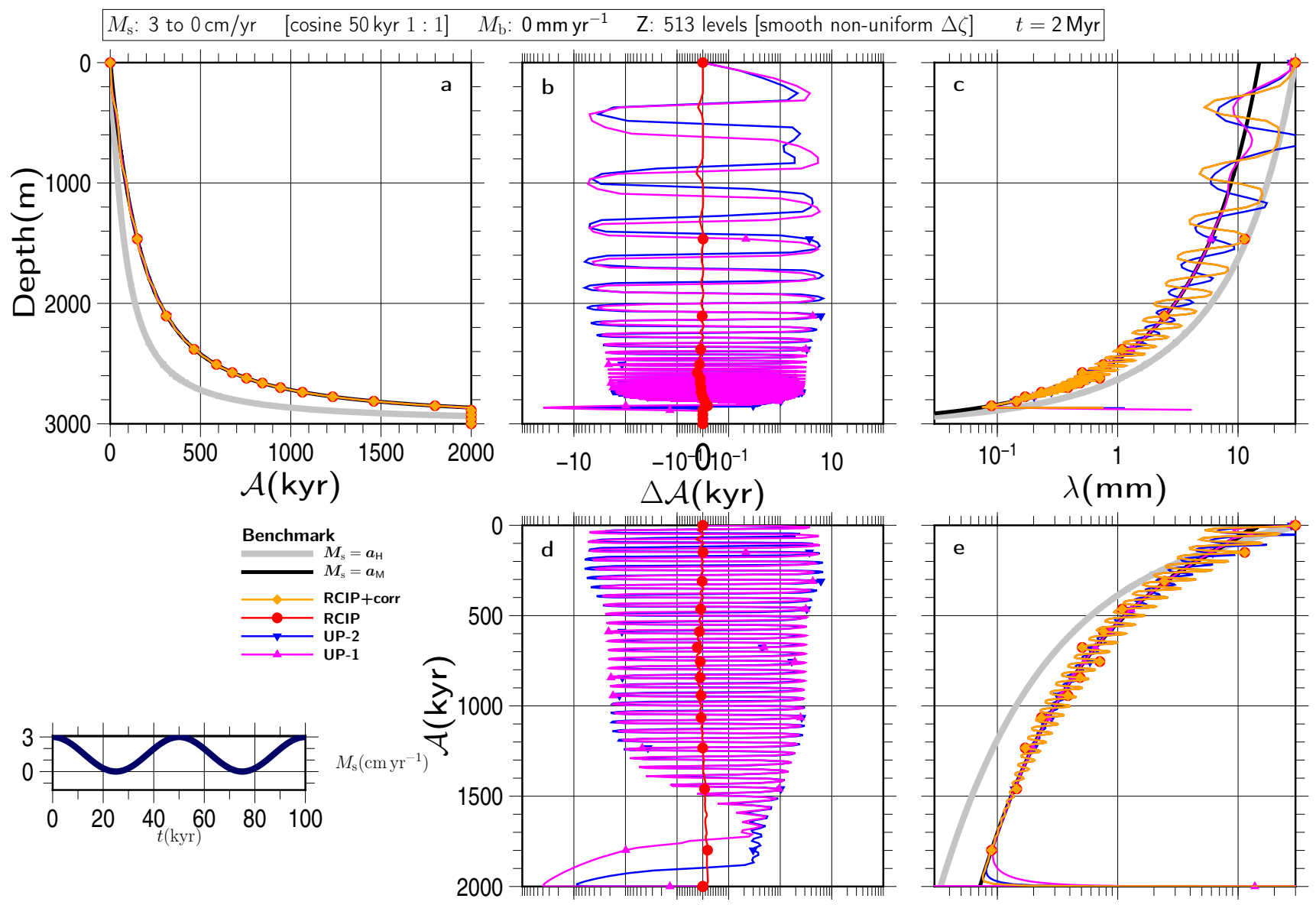

Figure S108 

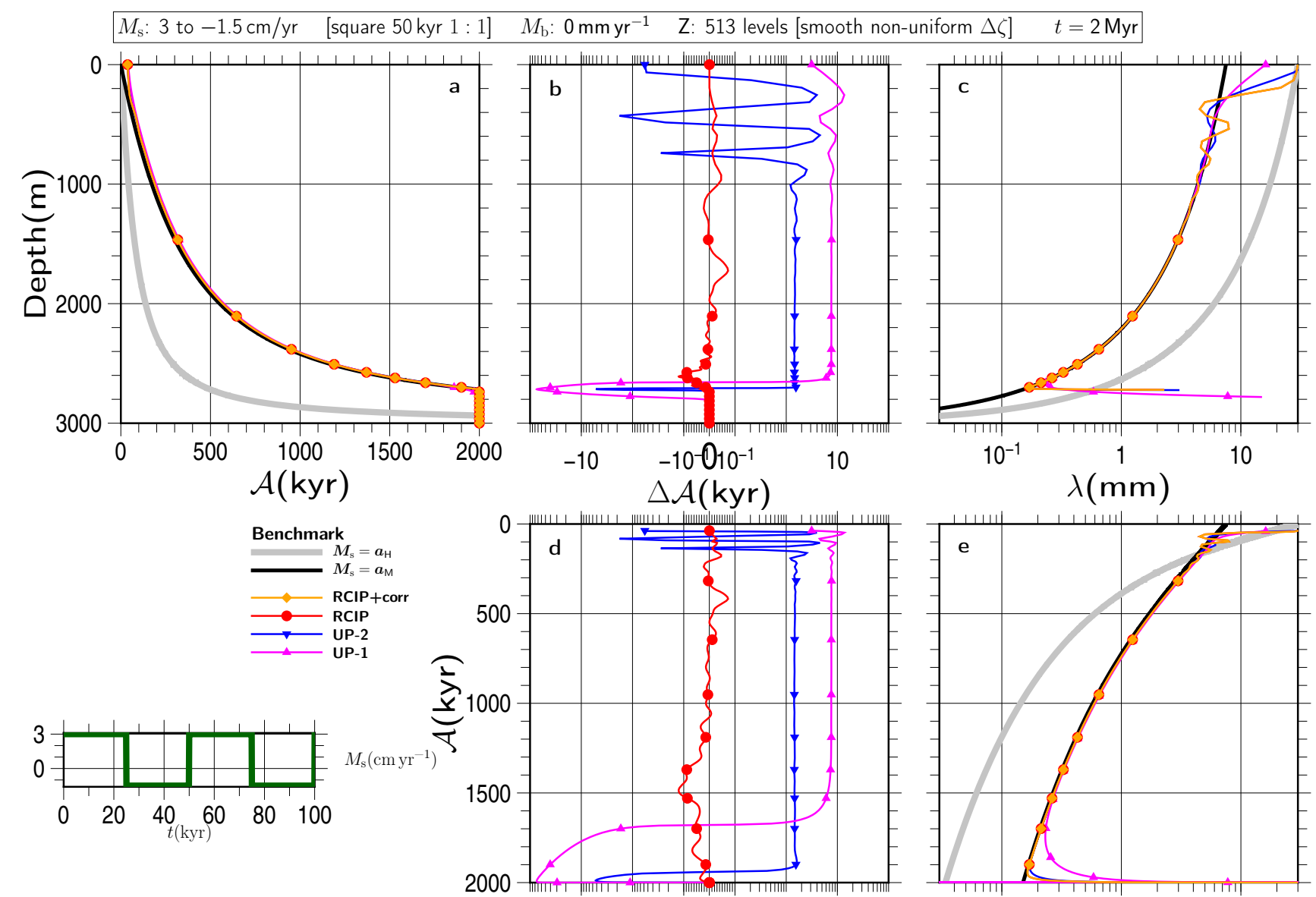

Figure S109
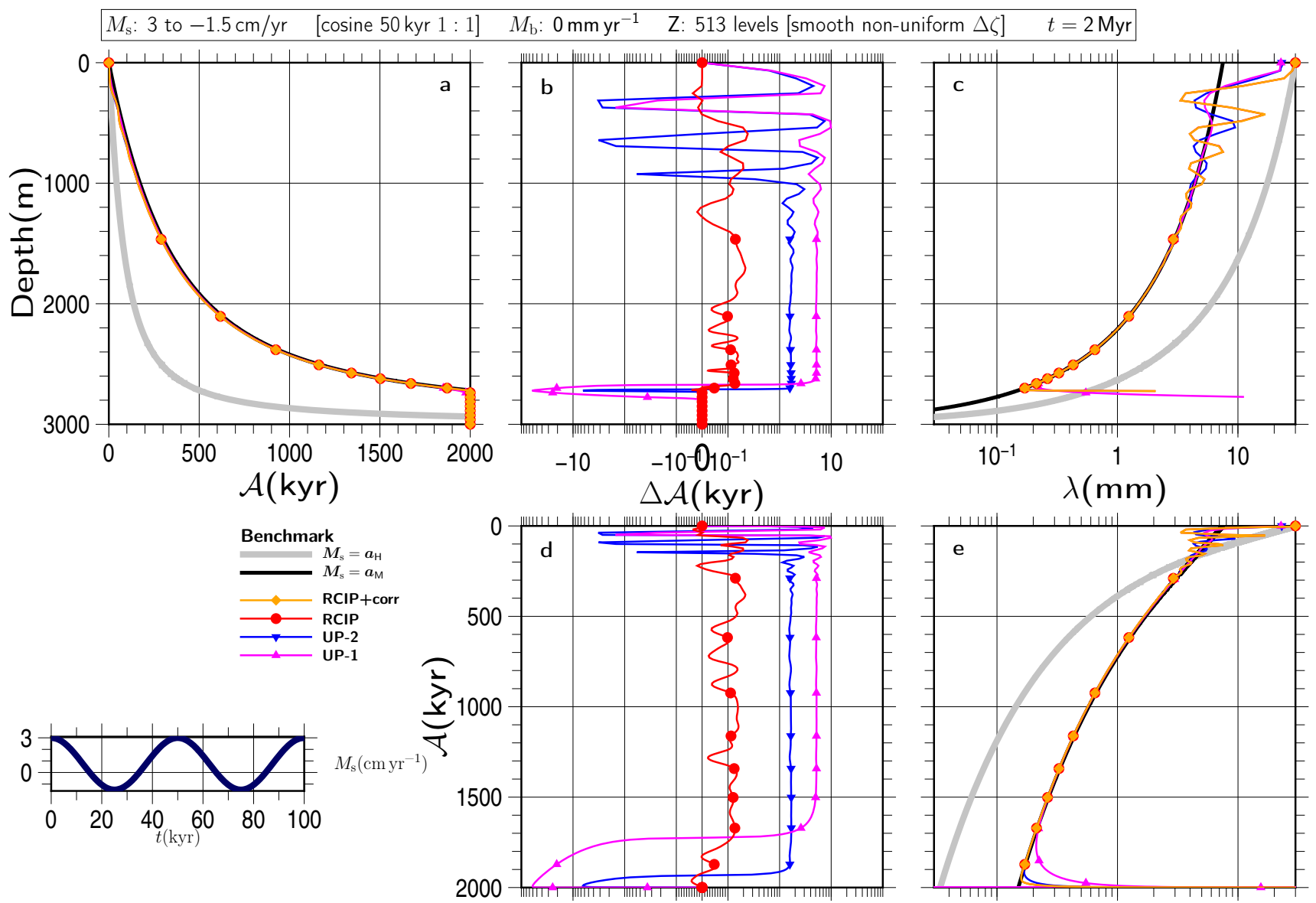

Figure S110 


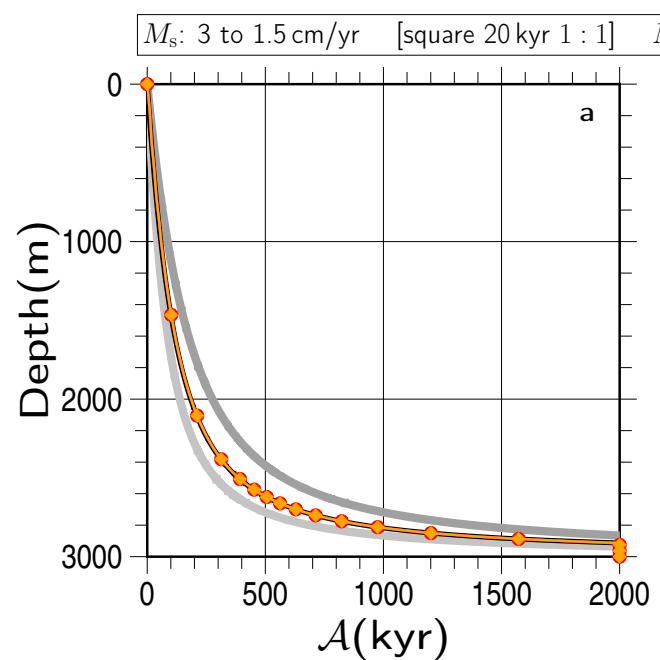

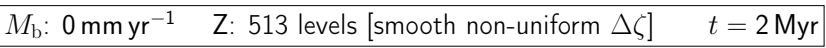
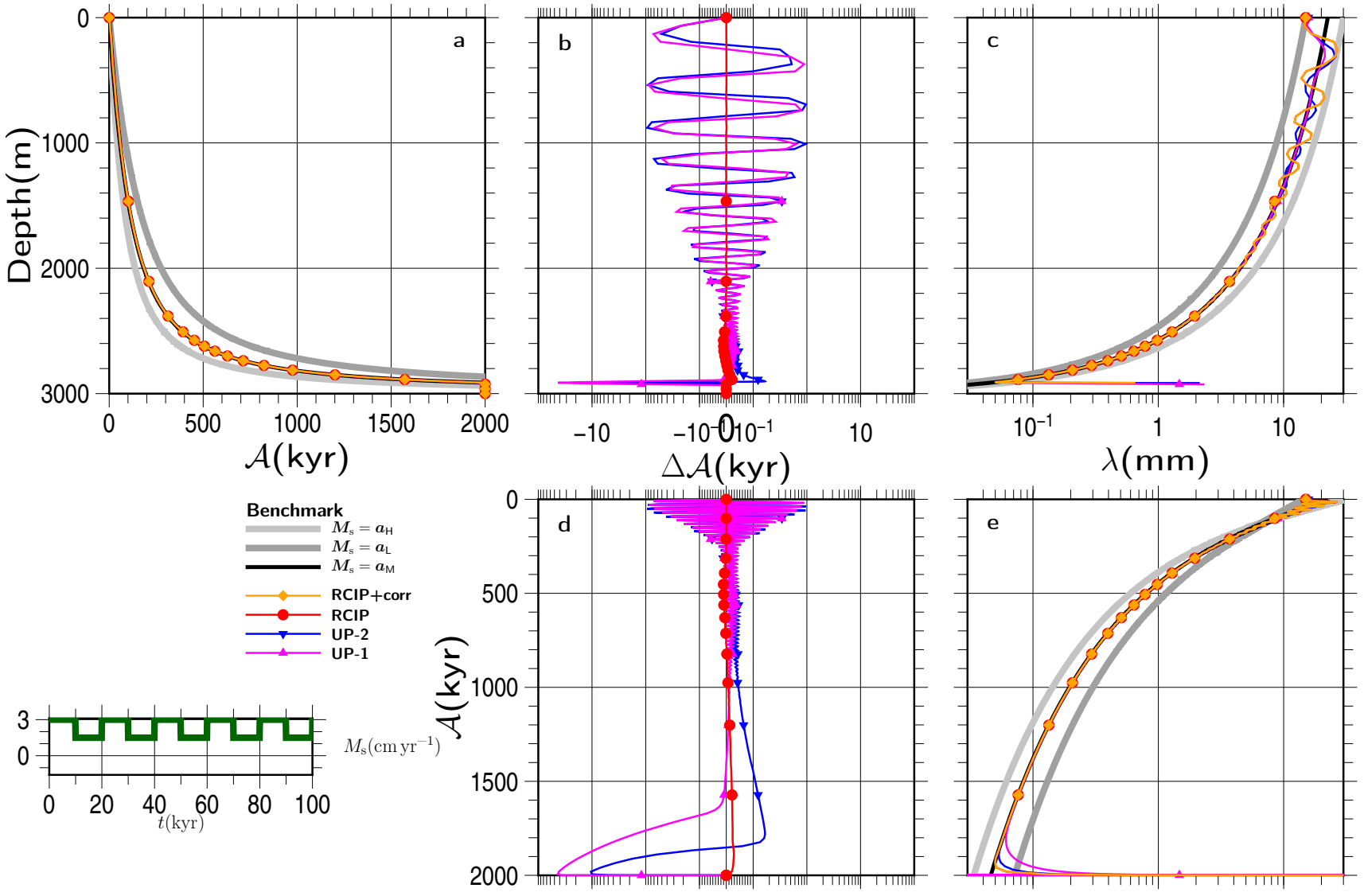

Figure S111
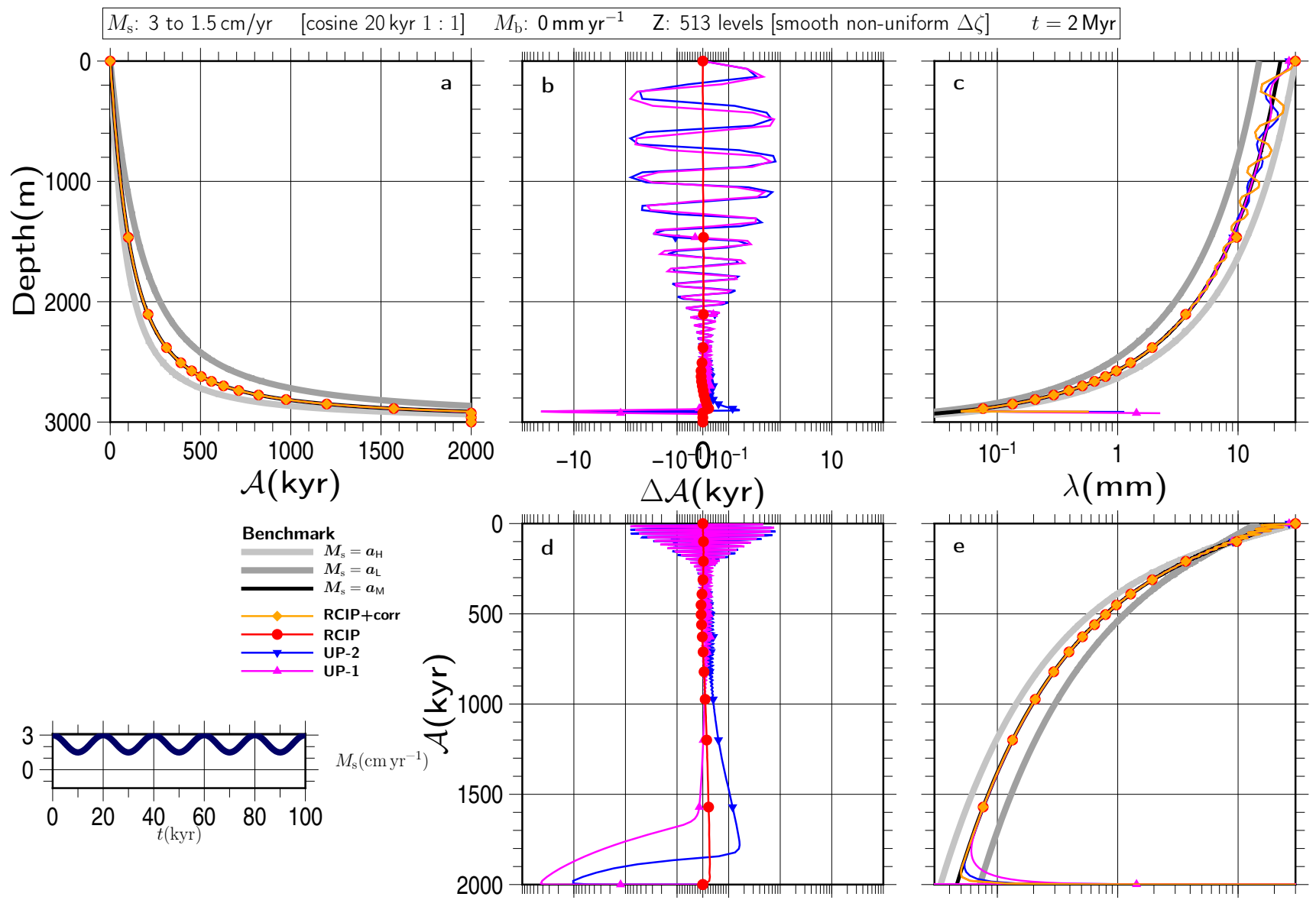

Figure S112 

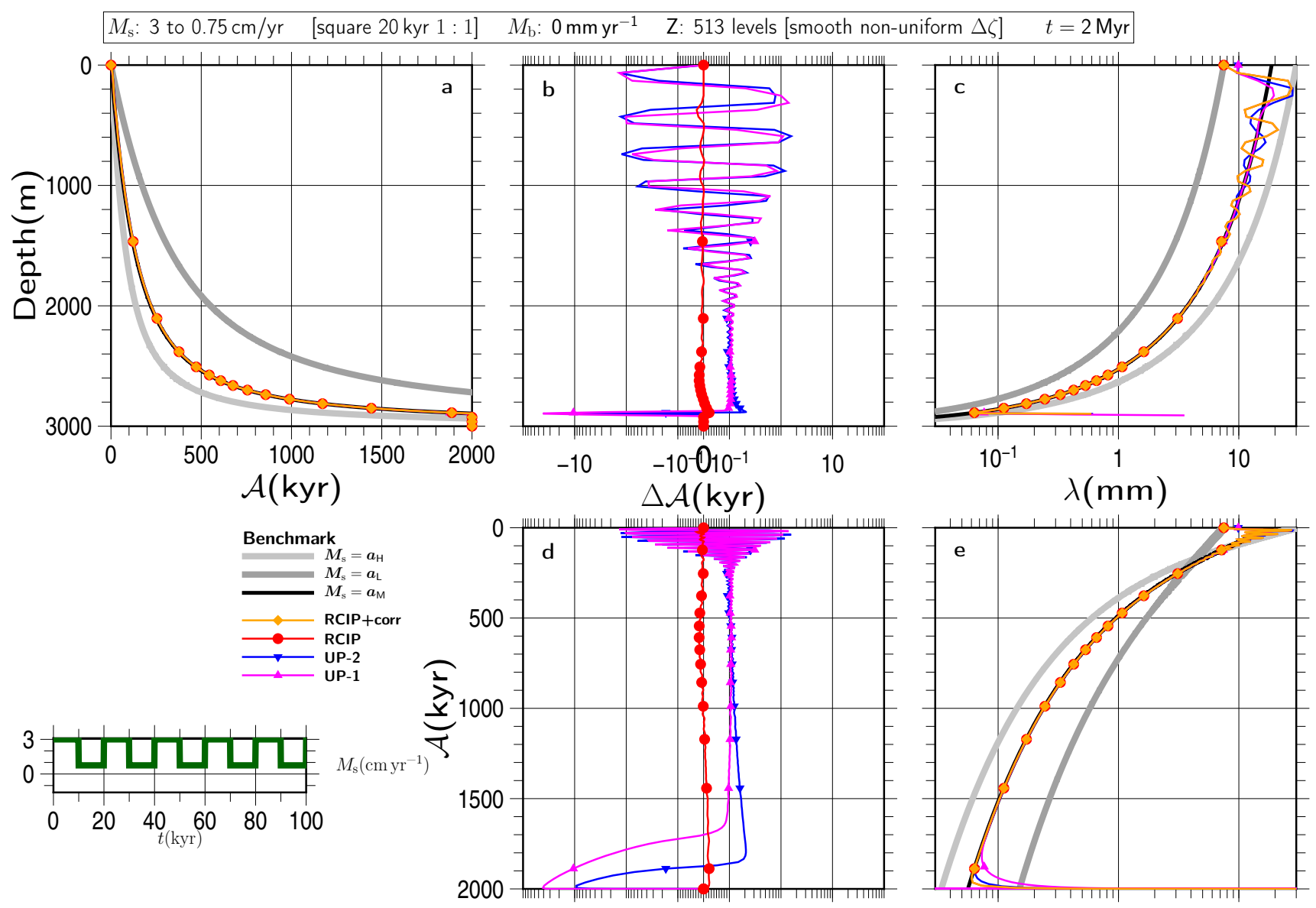

Figure S113
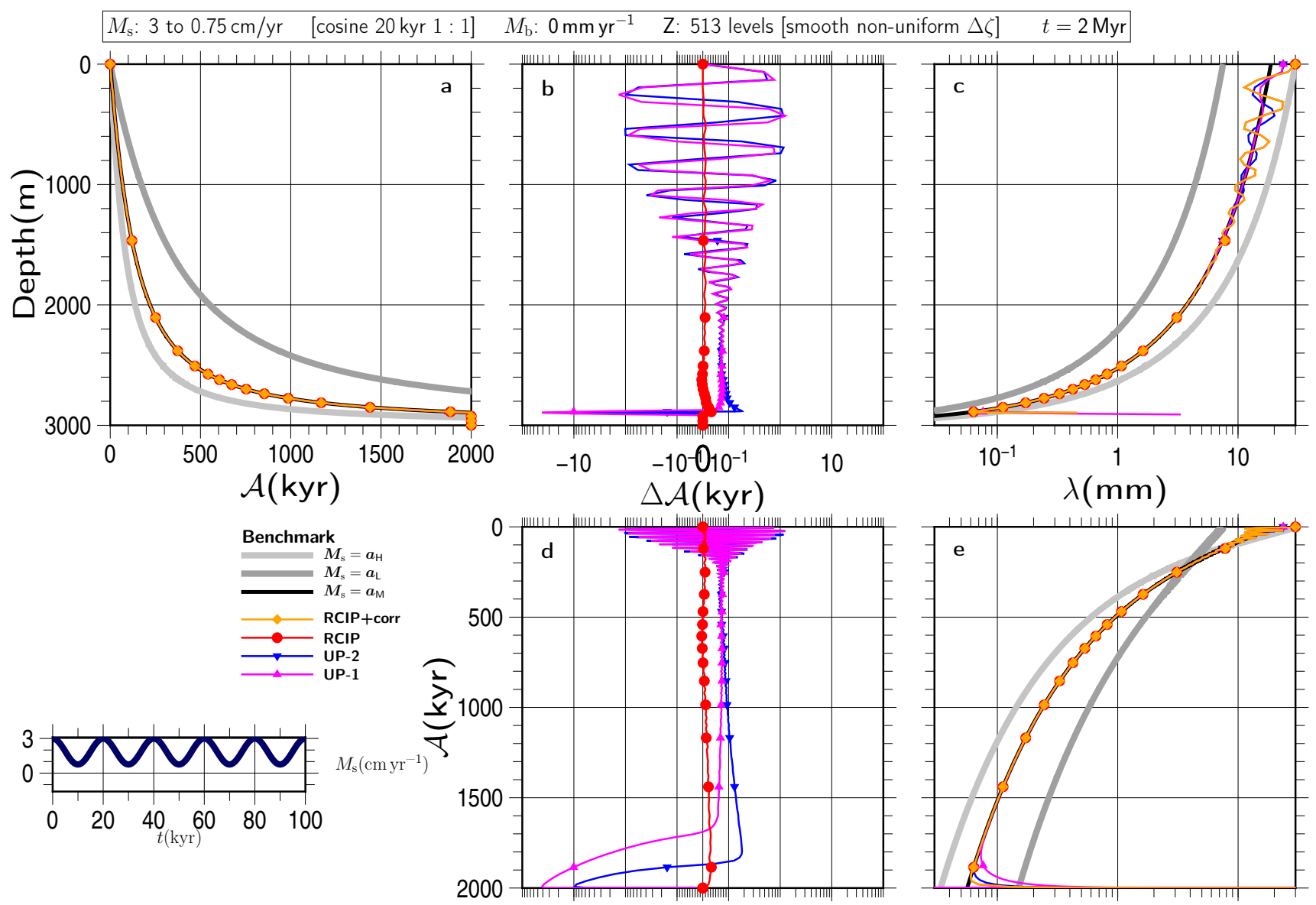

Figure S114 

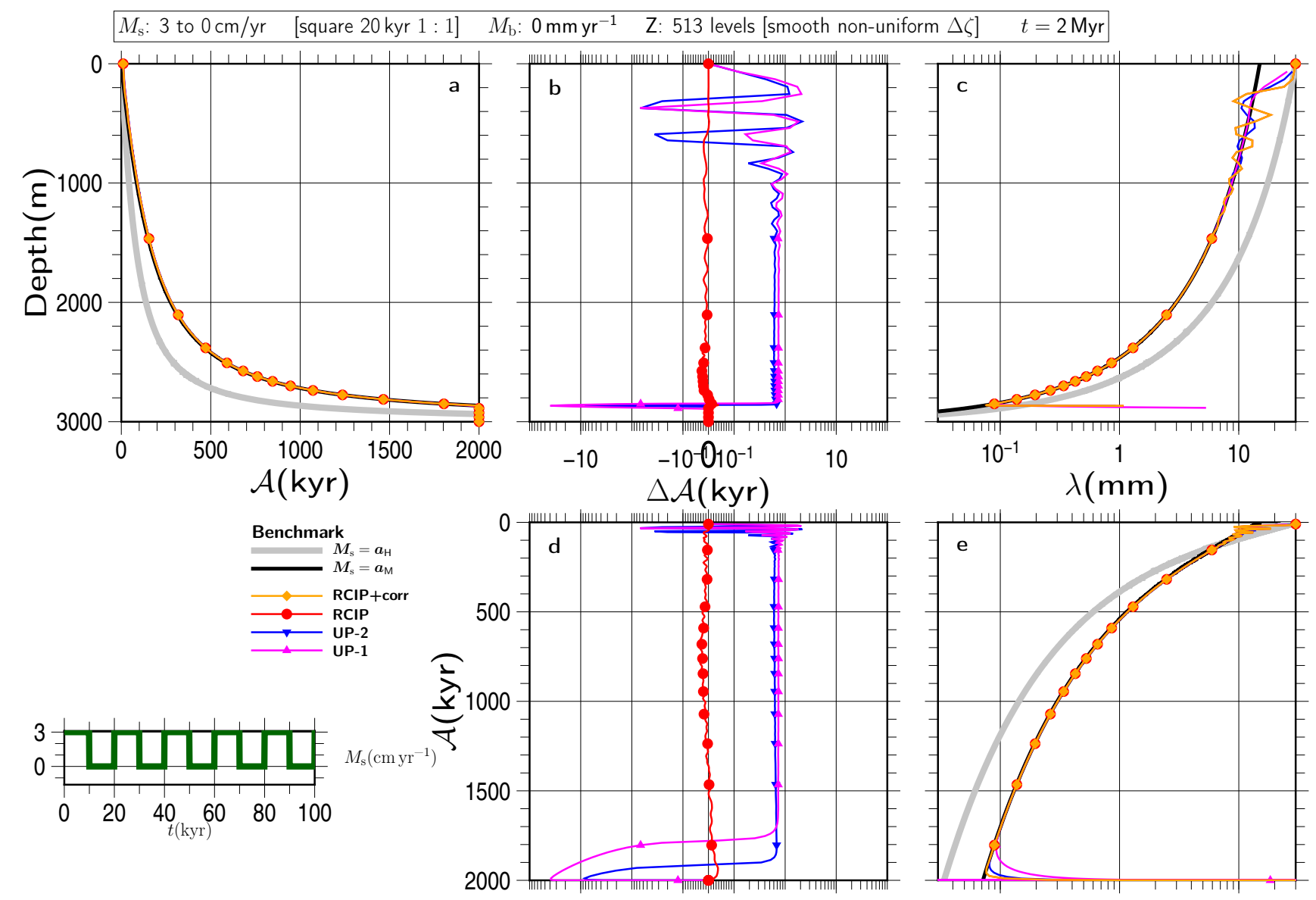

Figure S115
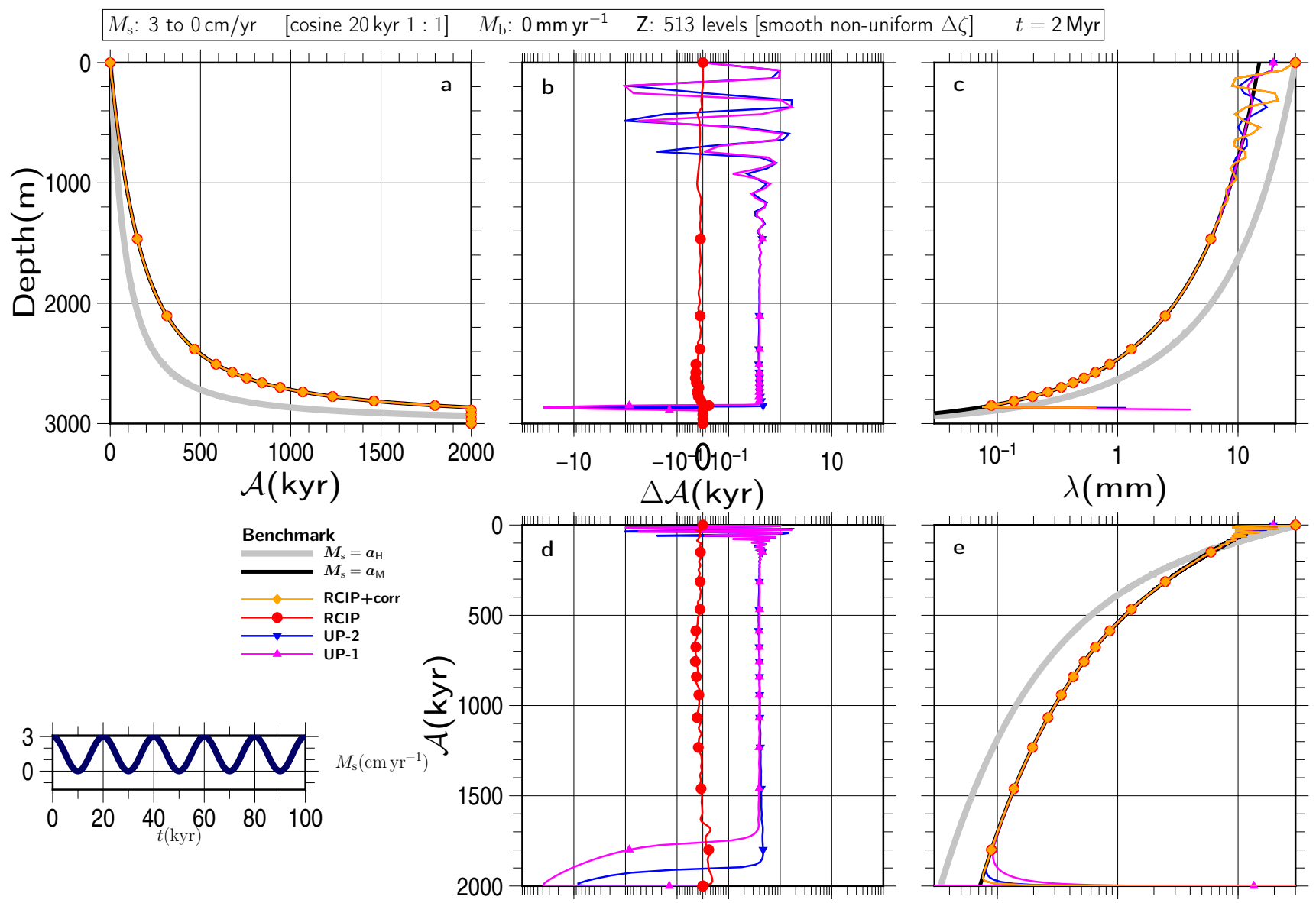

Figure S116 

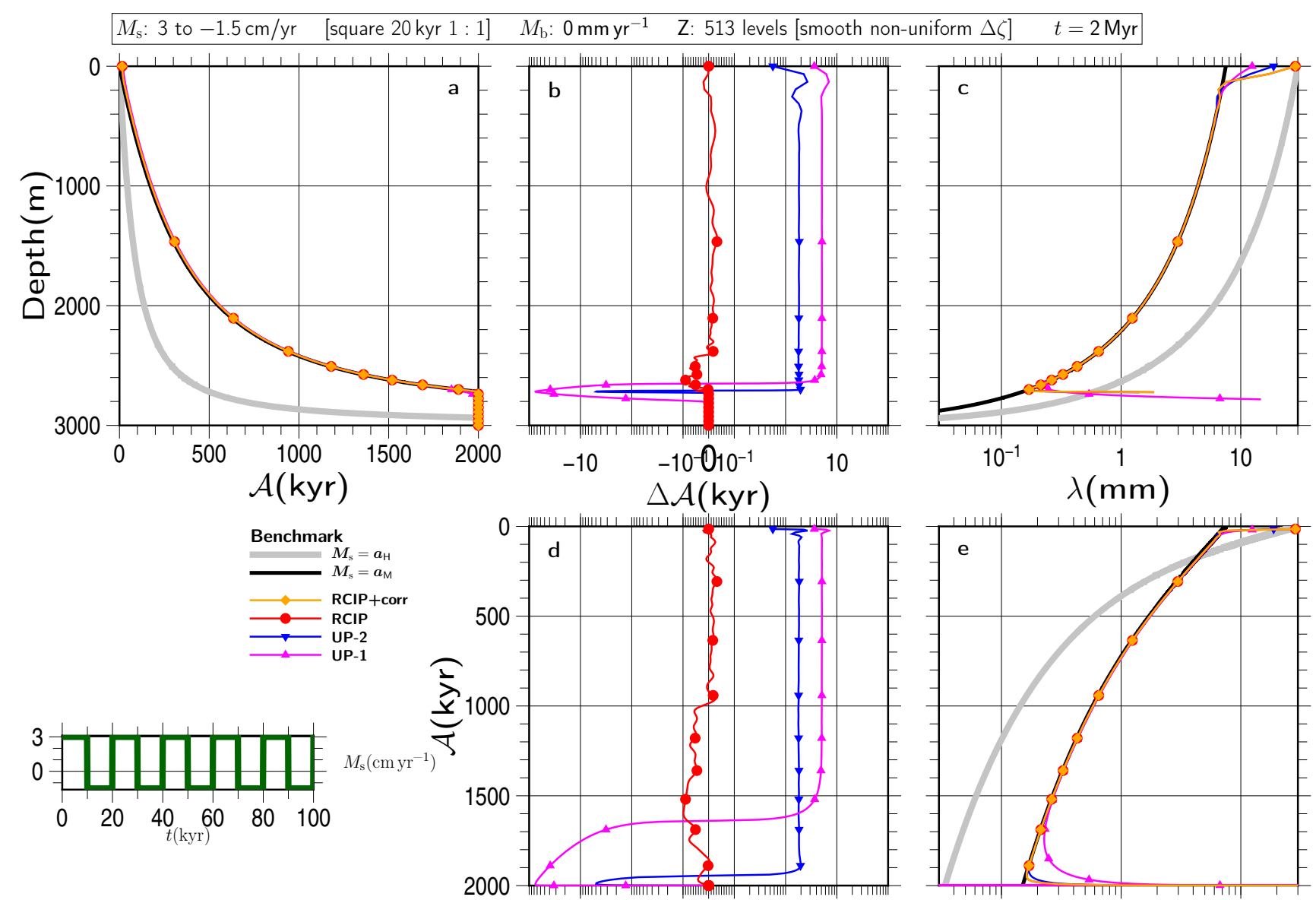

Figure S117
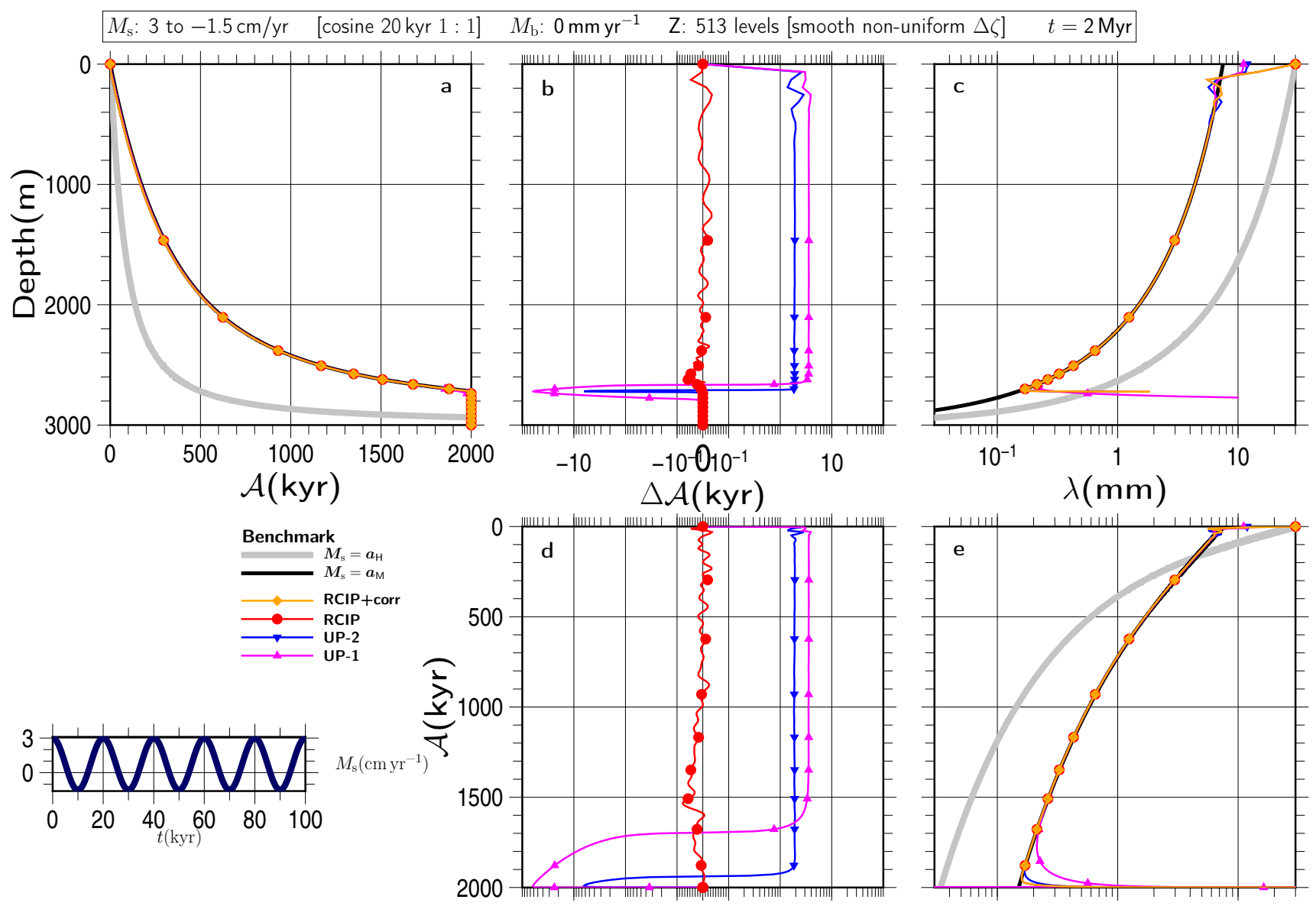

Figure S118 


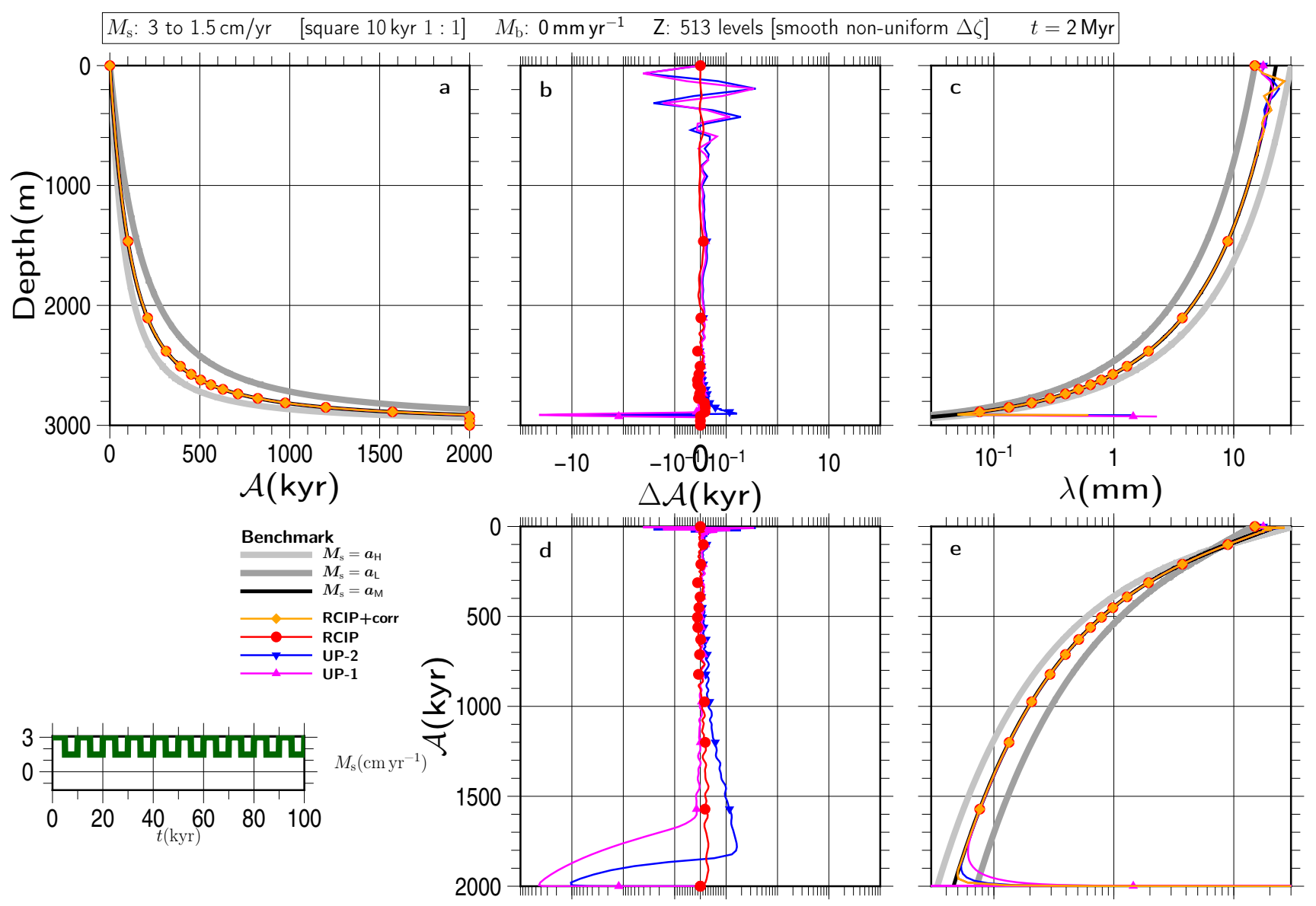

Figure S119

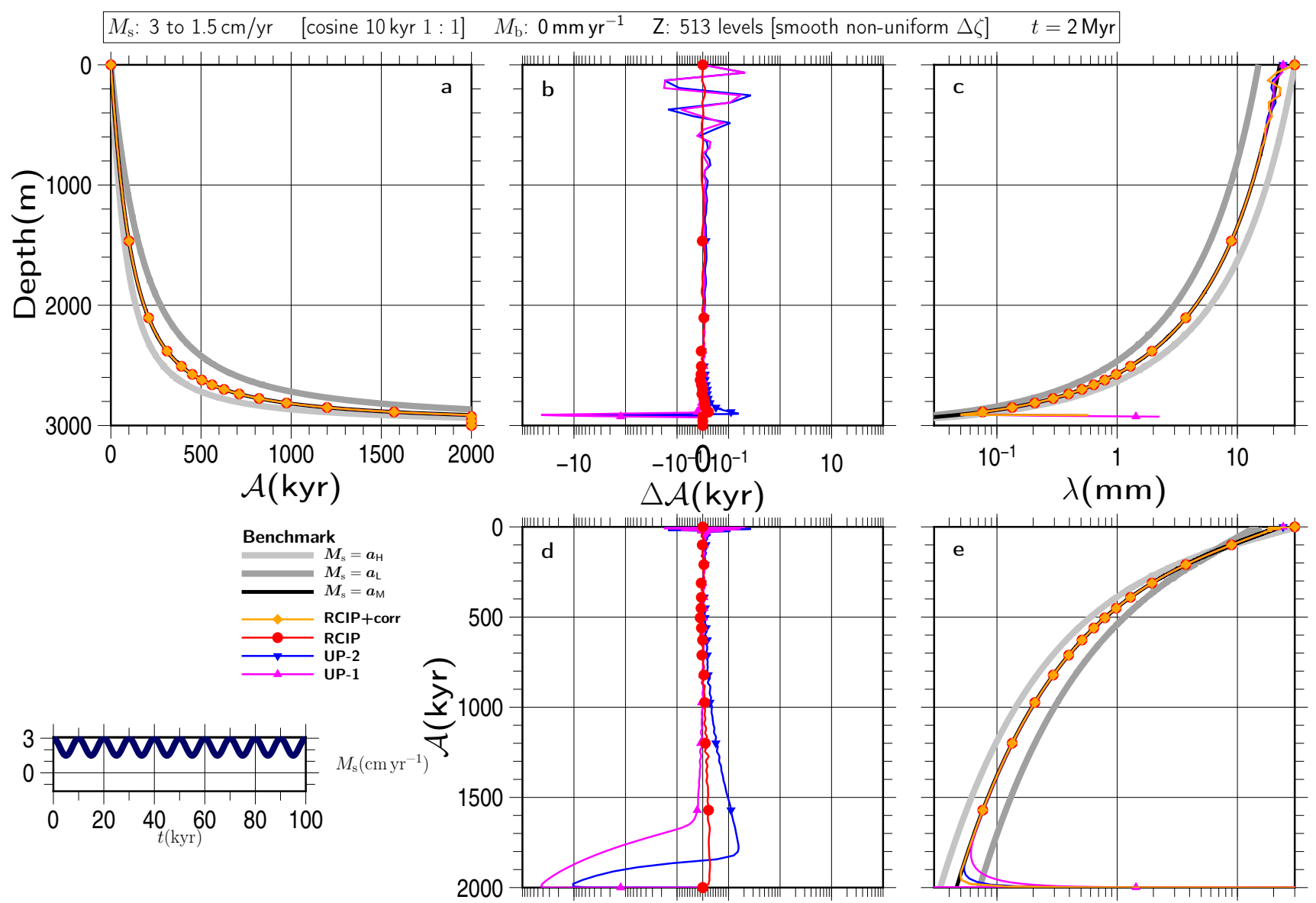

Figure S120 

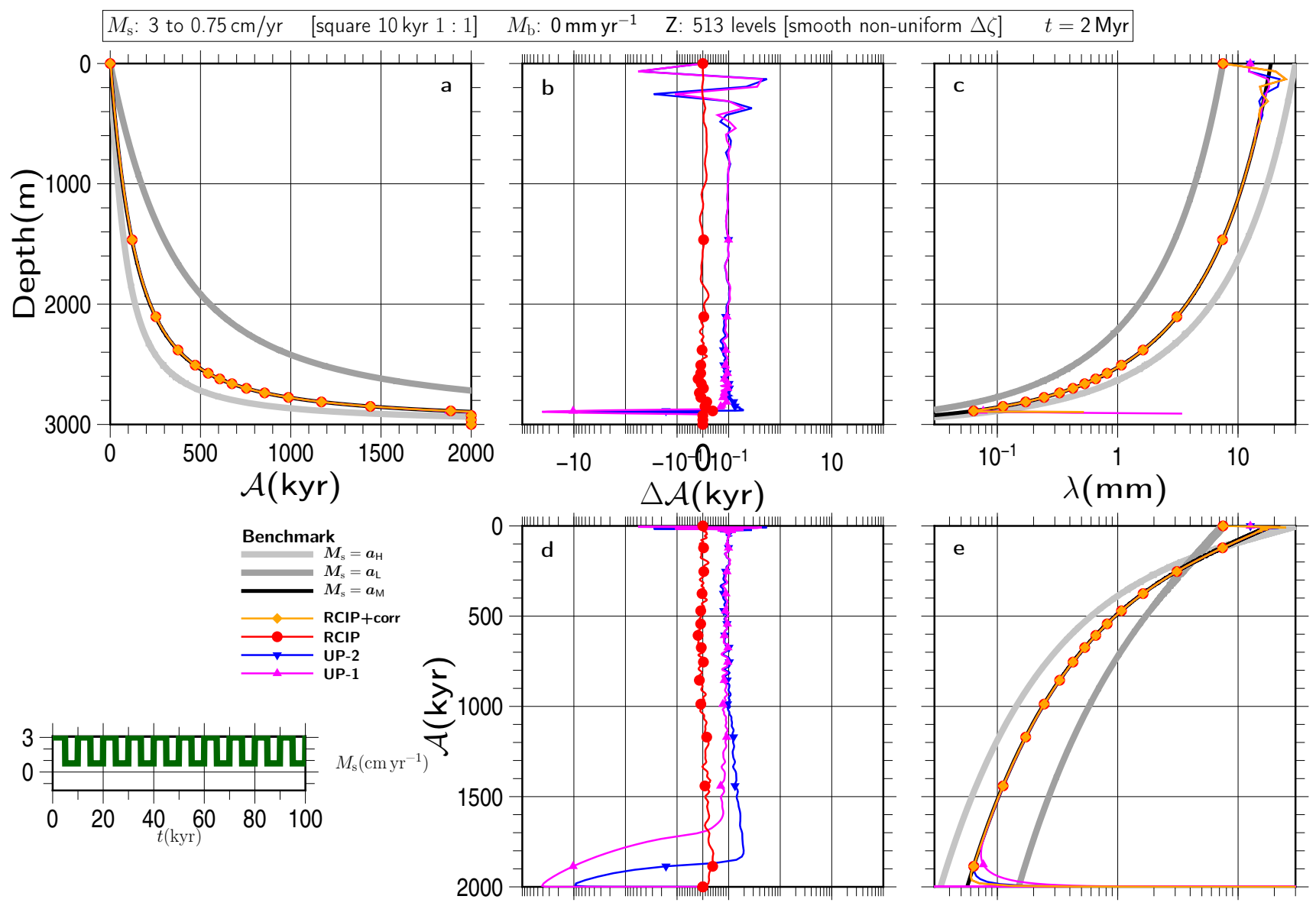

Figure S121
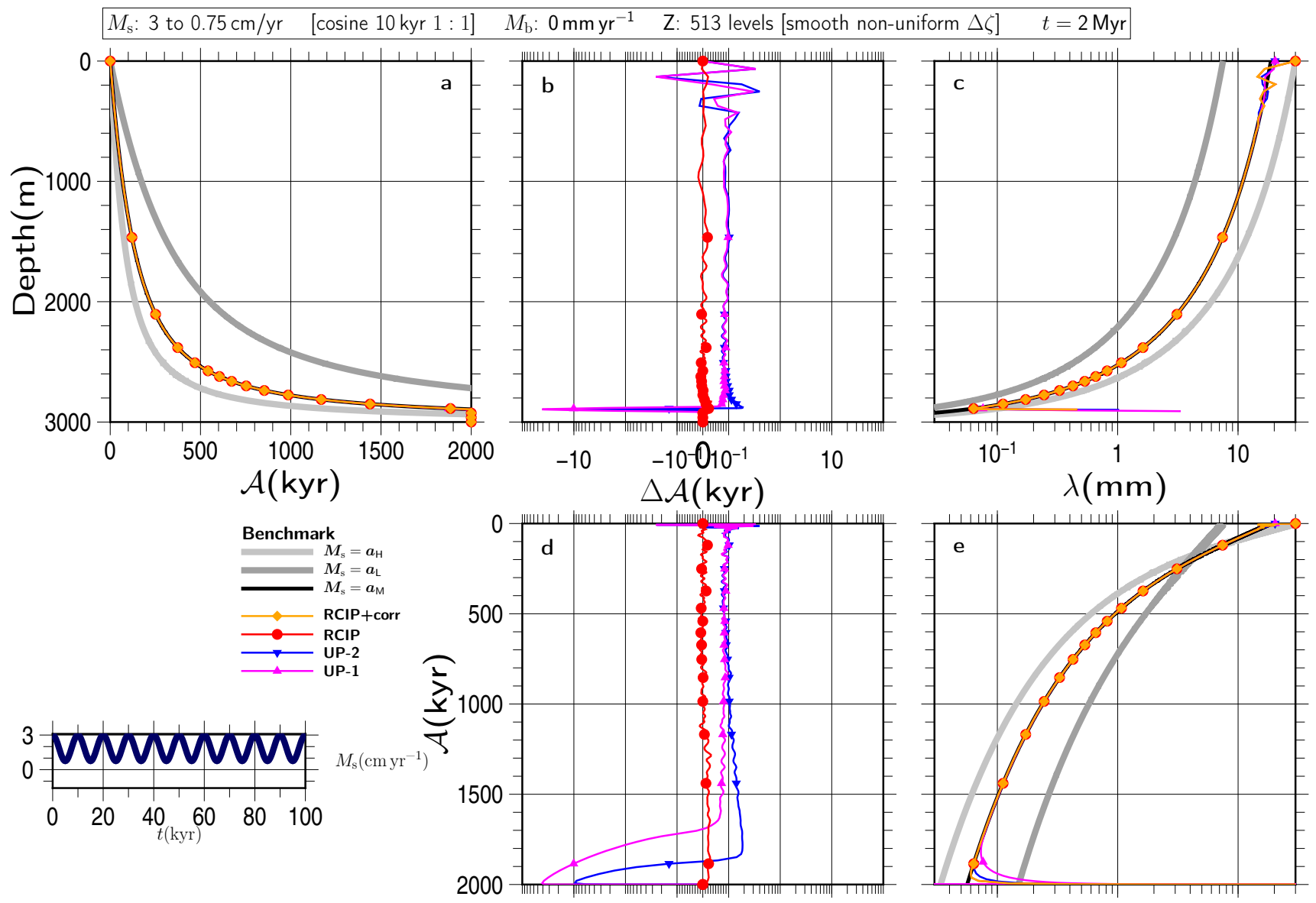

Figure S122 


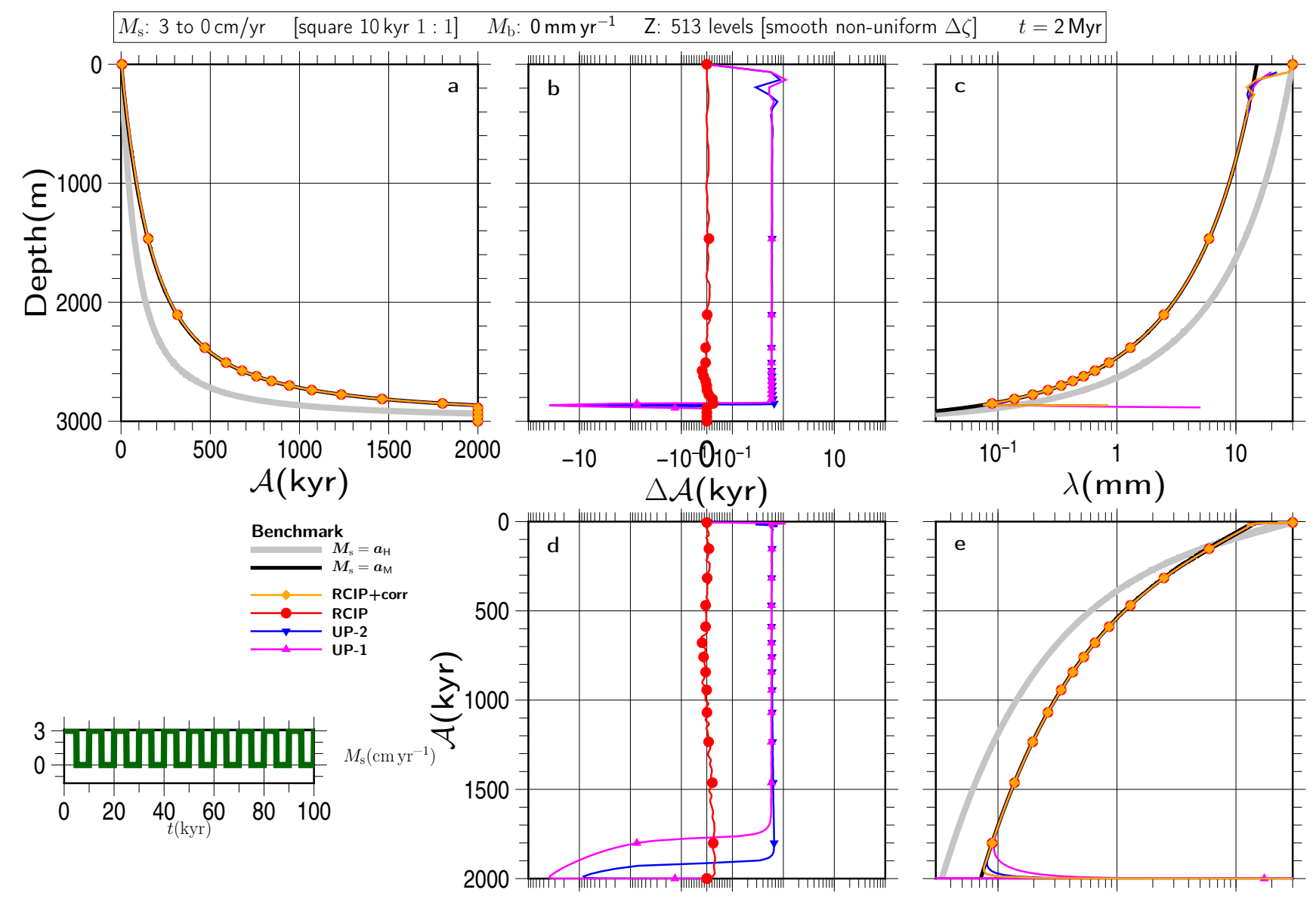

Figure S123

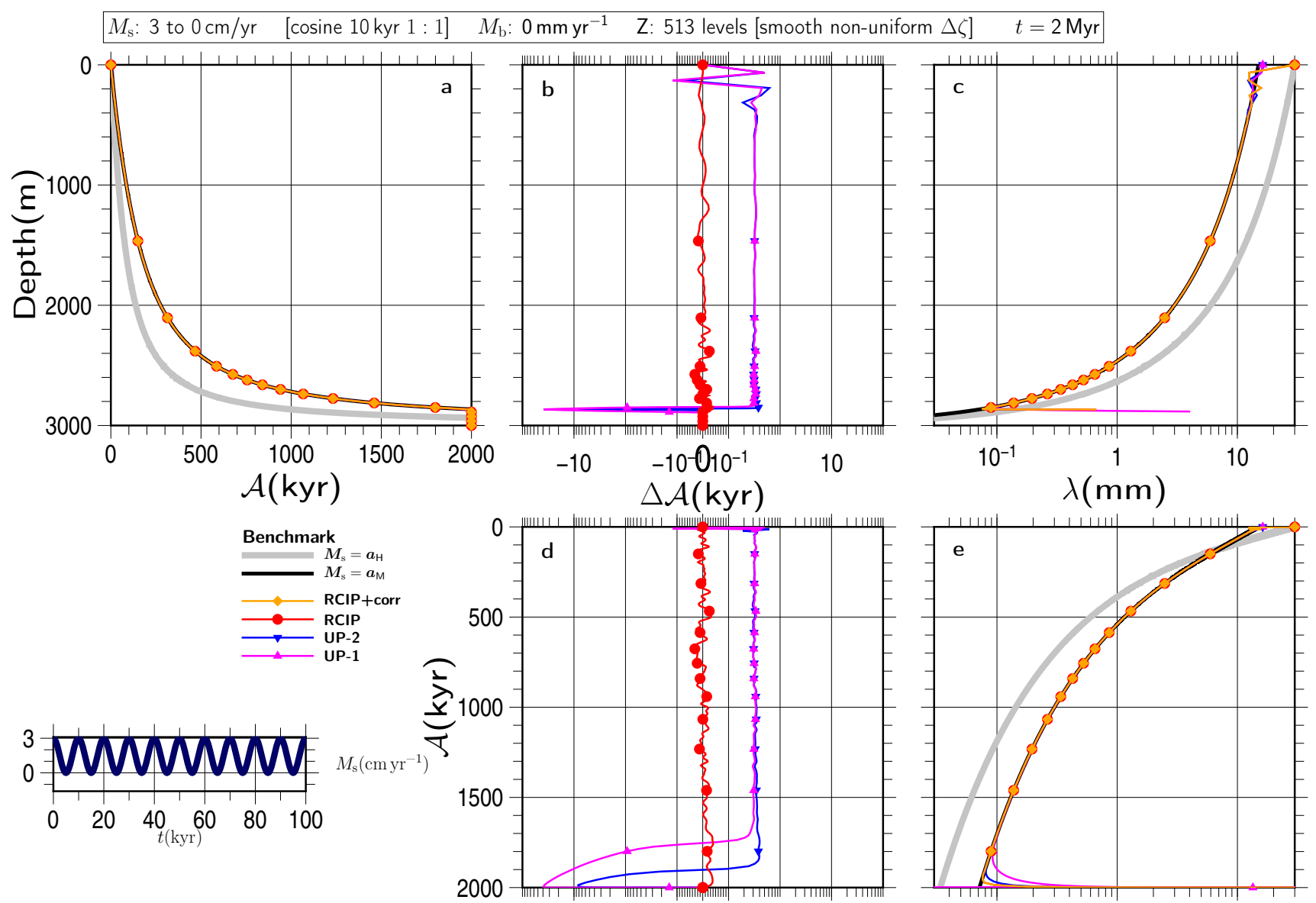

Figure S124 

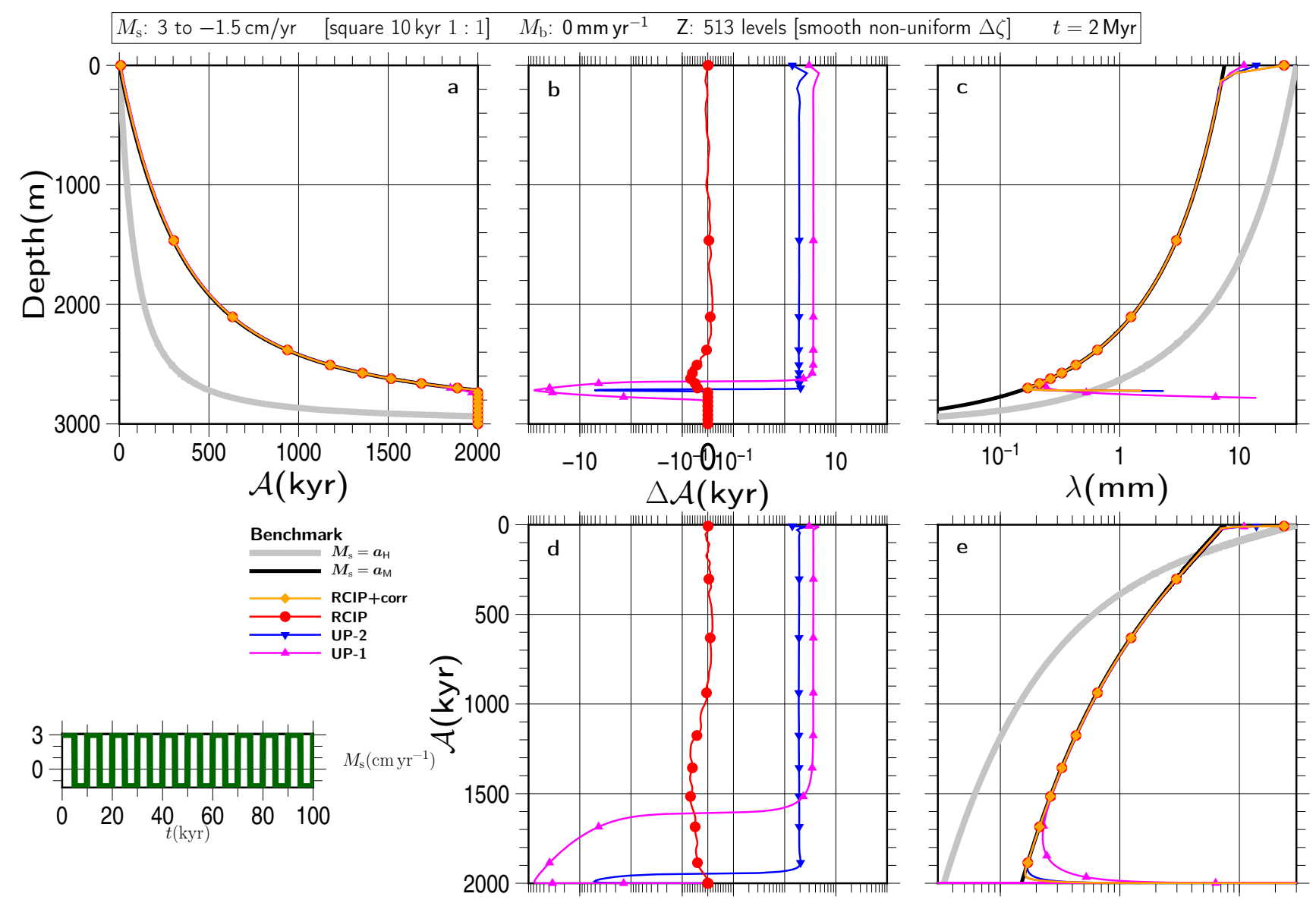

Figure S125

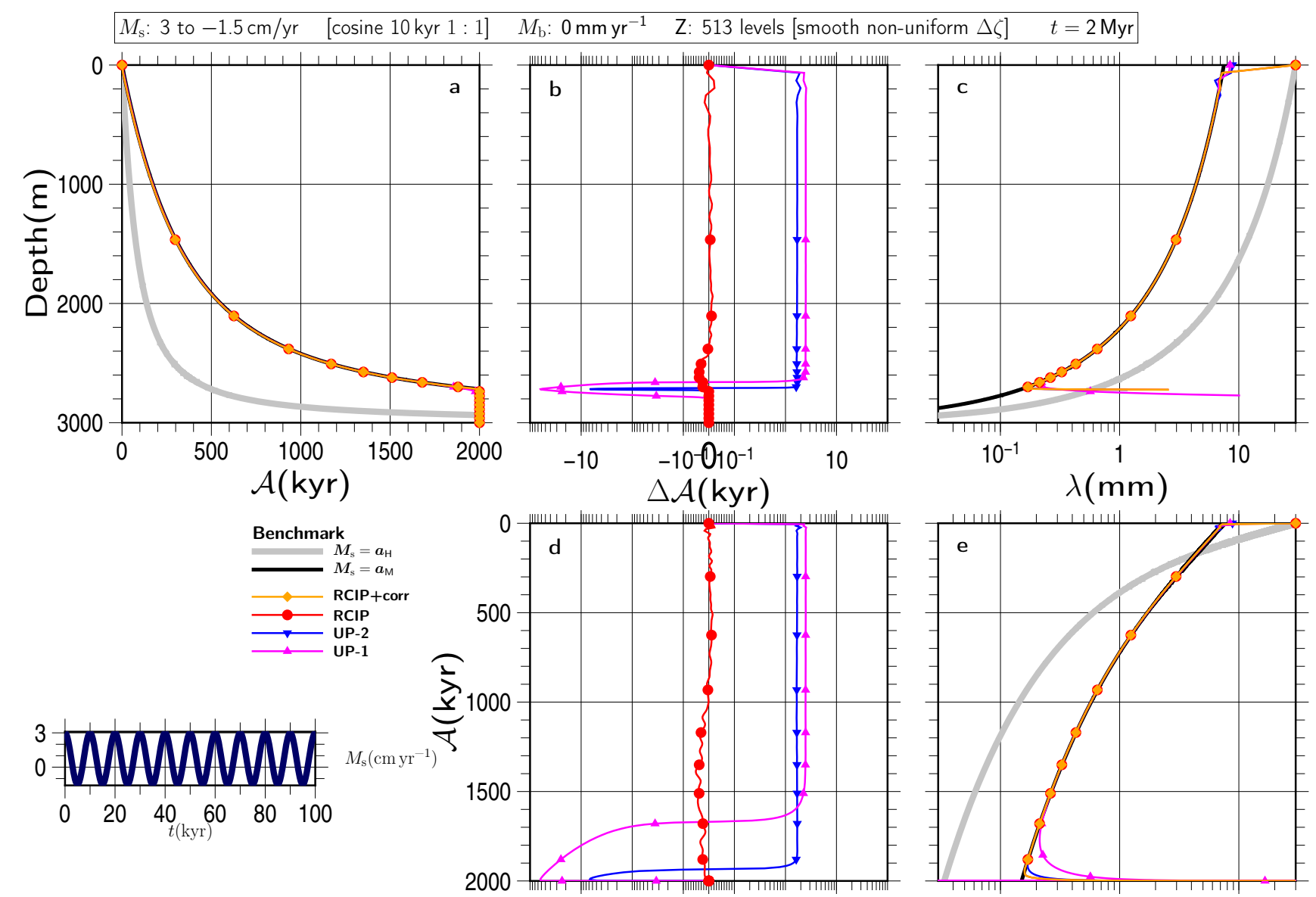

Figure S126 

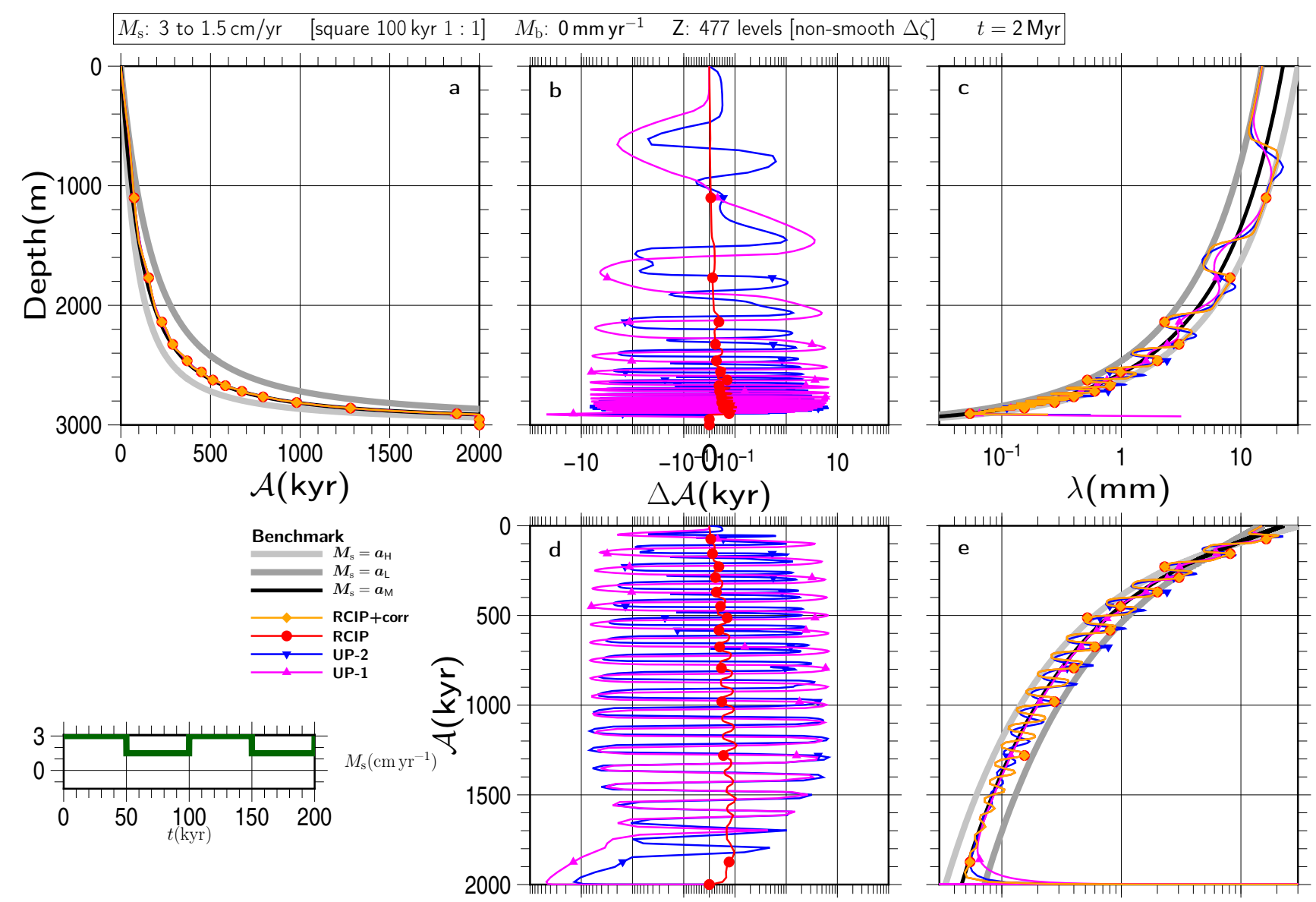

Figure S127
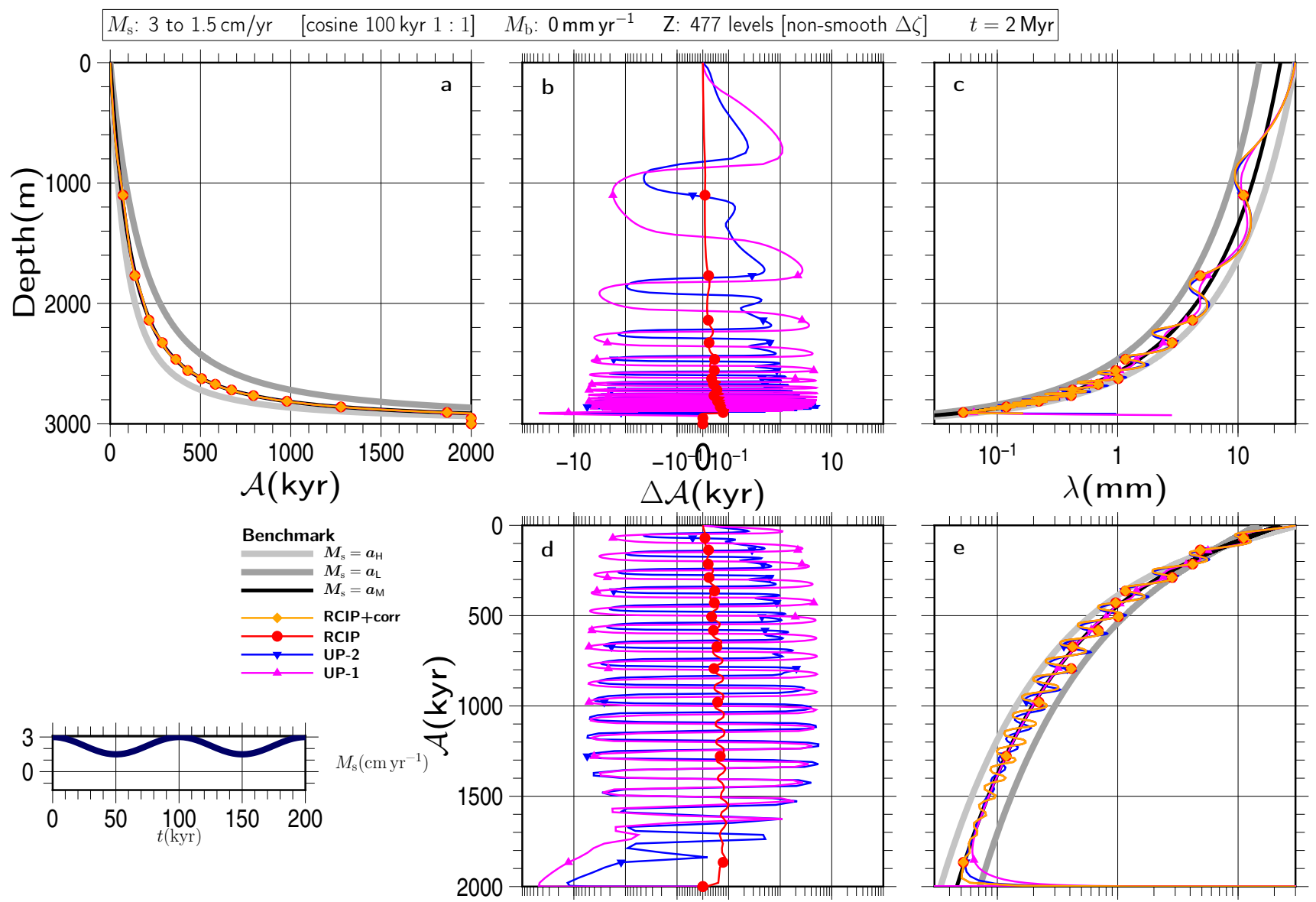

Figure S128 

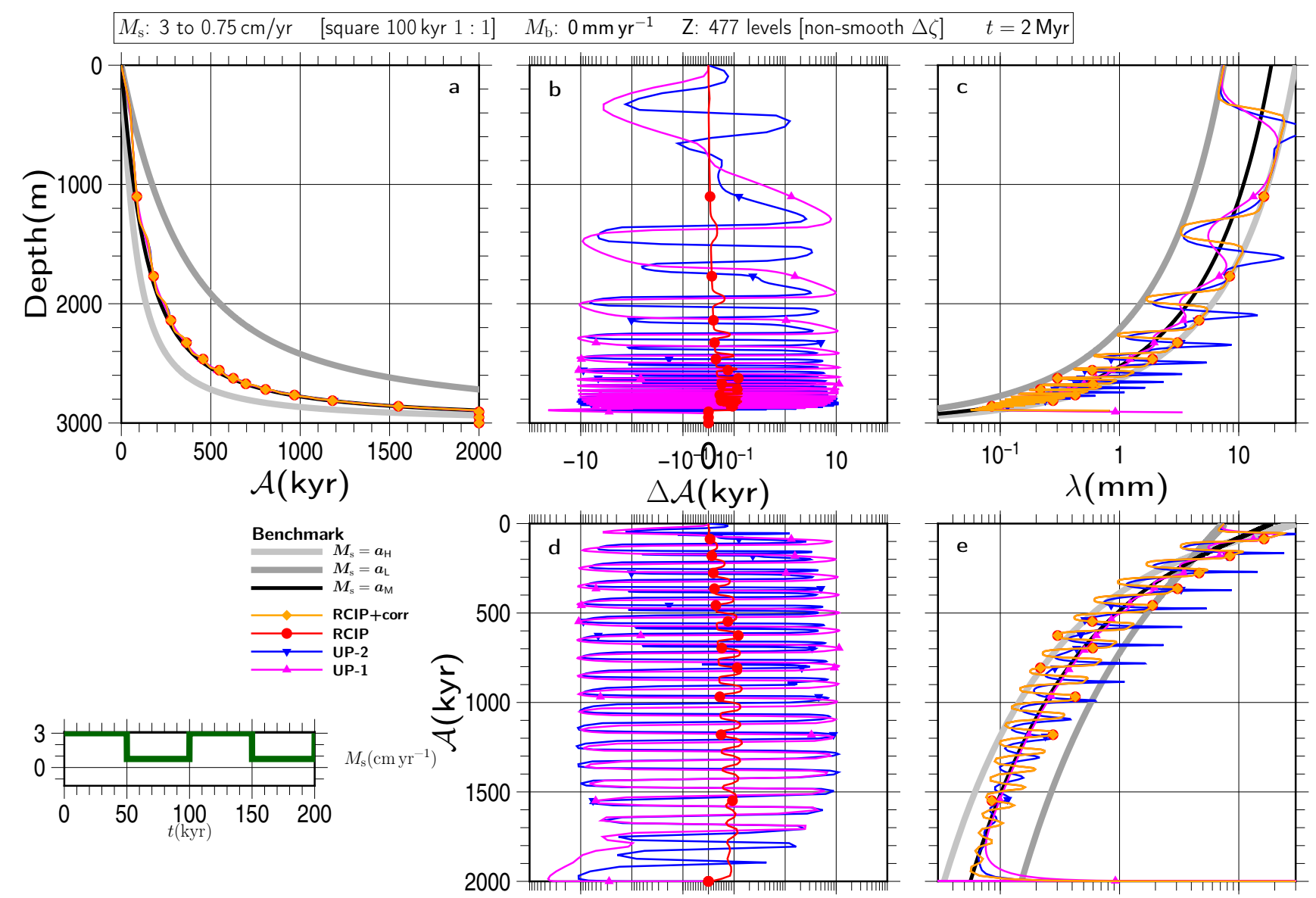

Figure S129
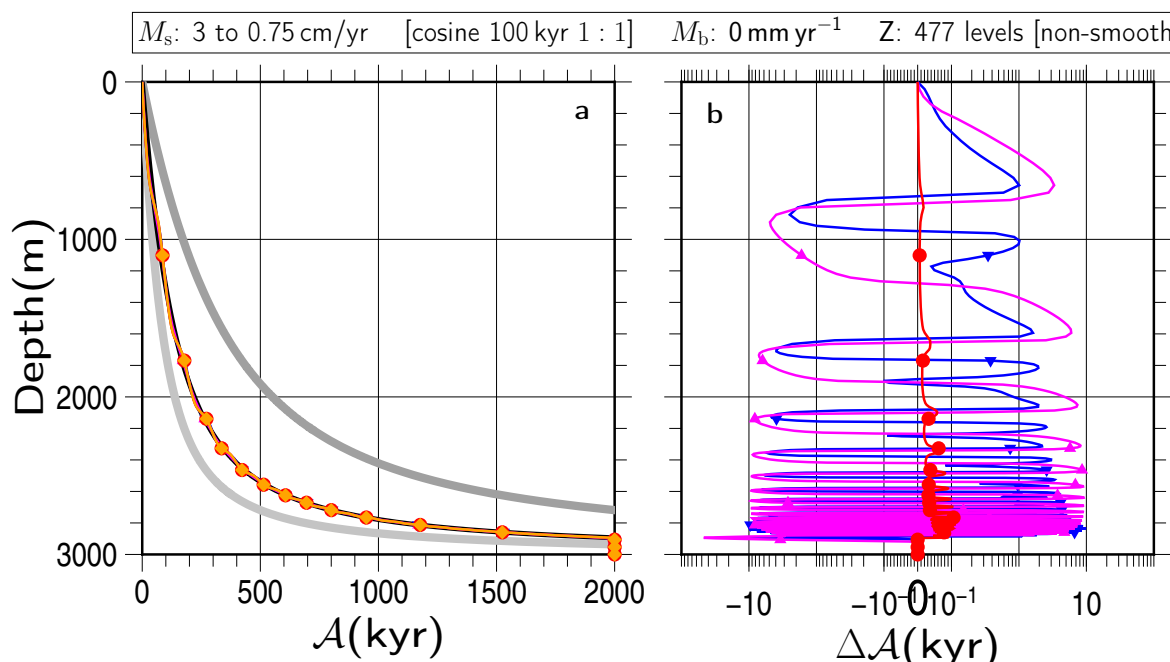

$\Delta \zeta] \quad t=2 \mathrm{Myr}$
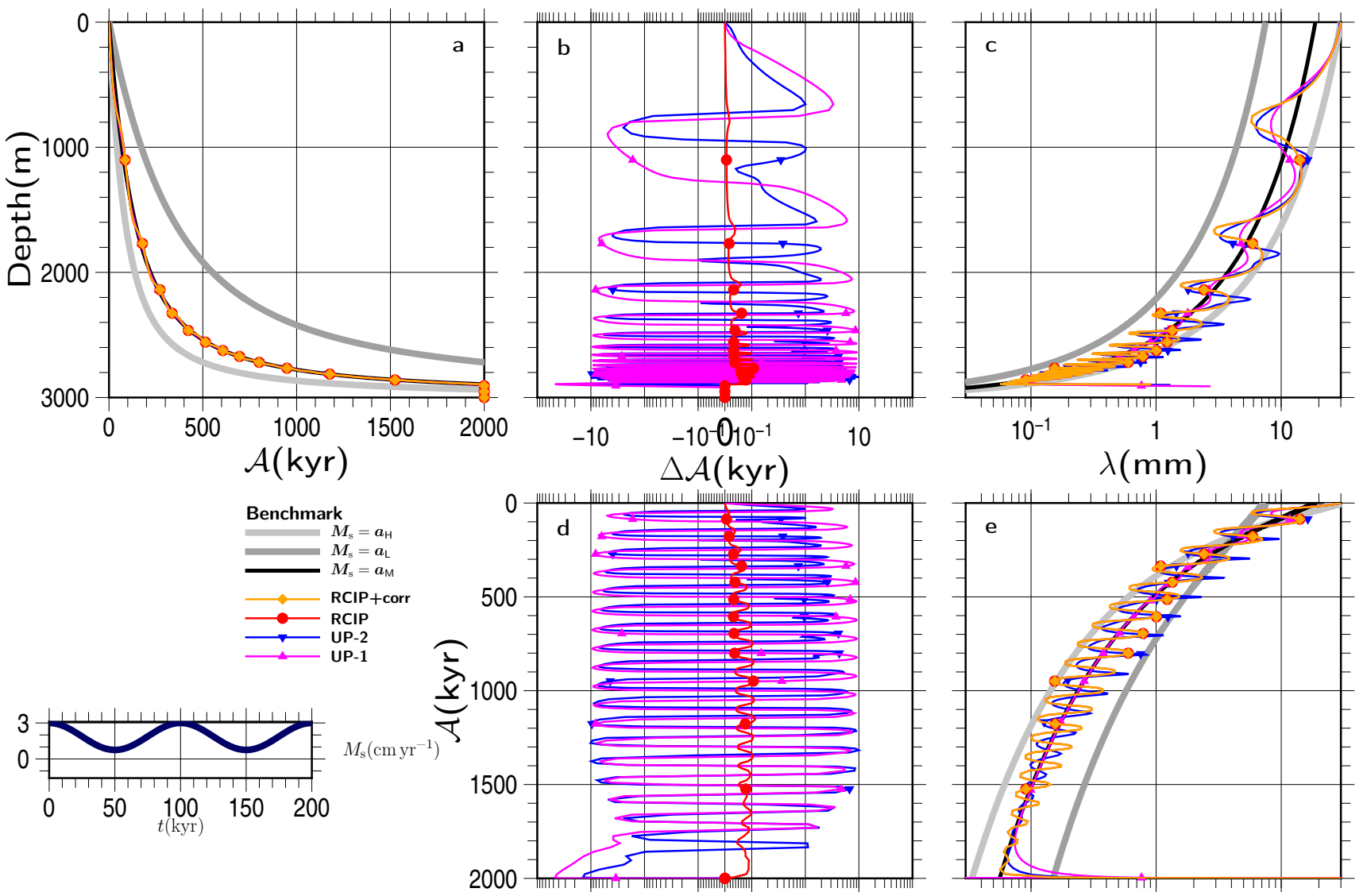

Figure S130 

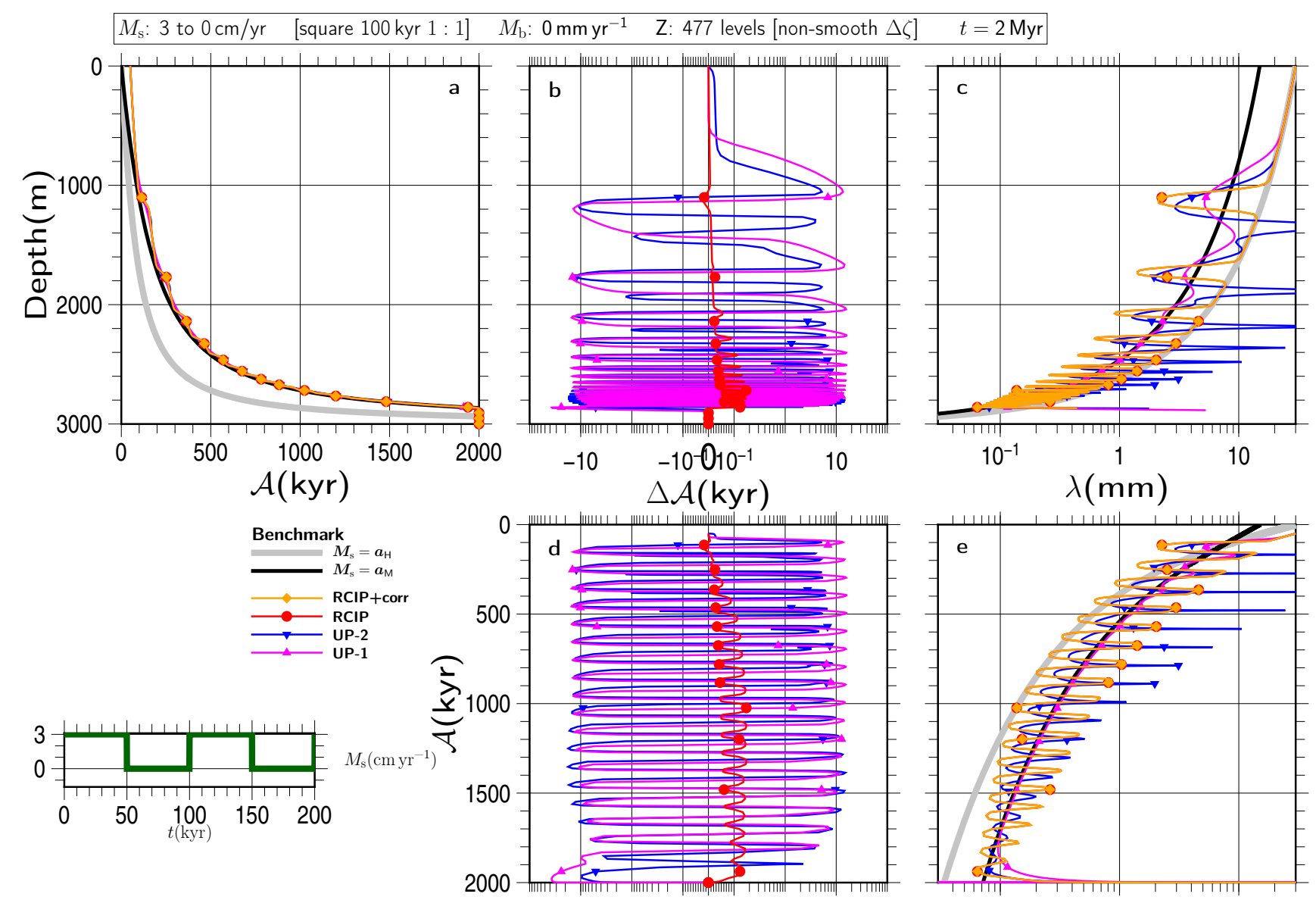

Figure S131
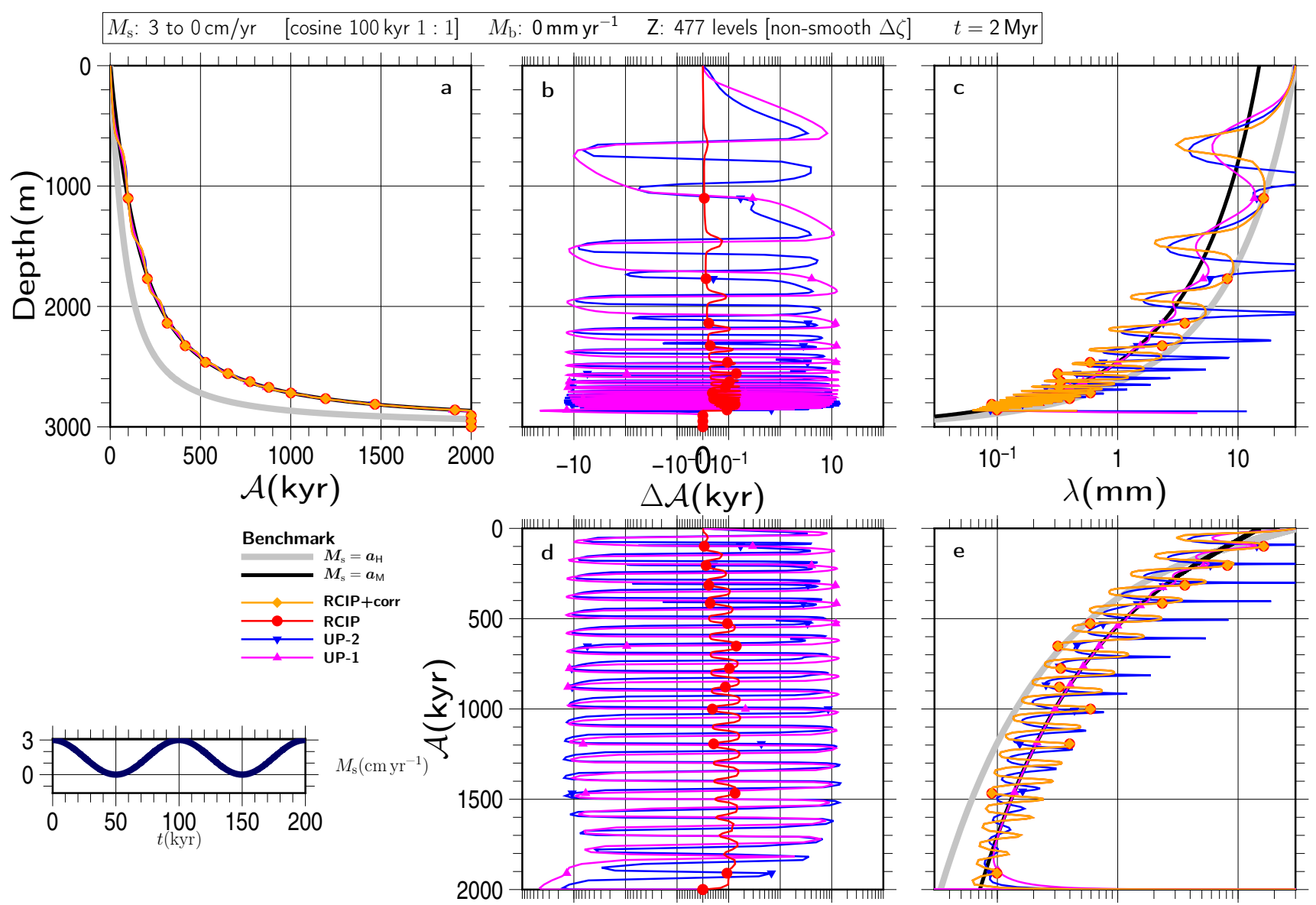

Figure S132 

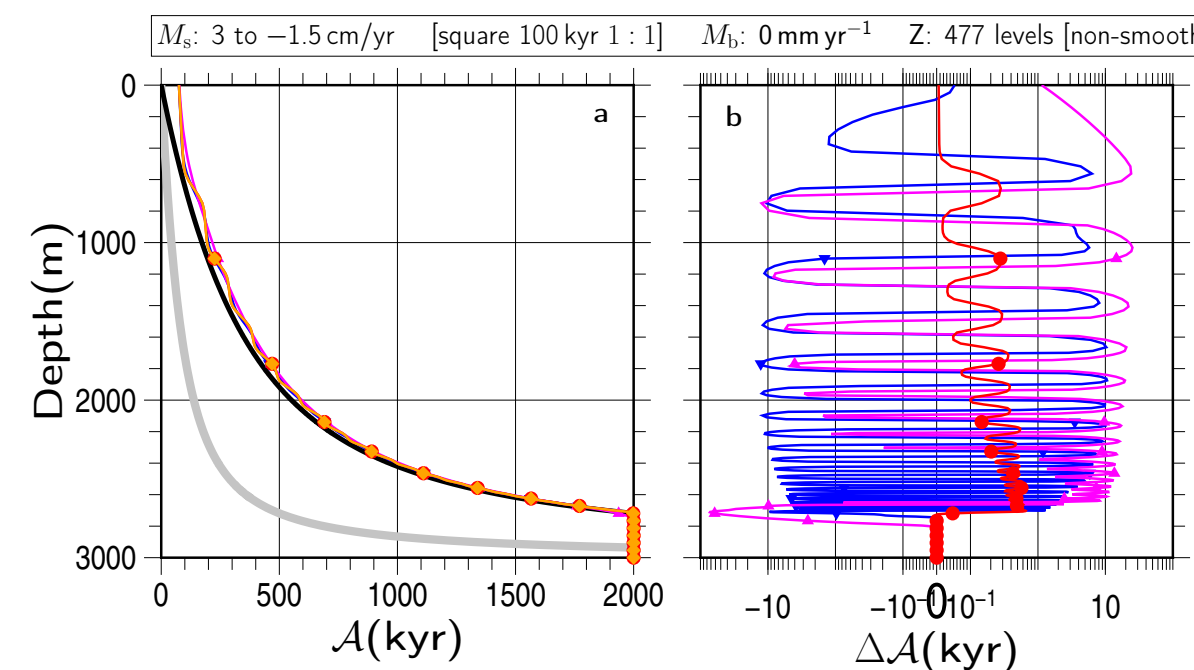

$\Delta \zeta] \quad t=2 \mathrm{Myr}$
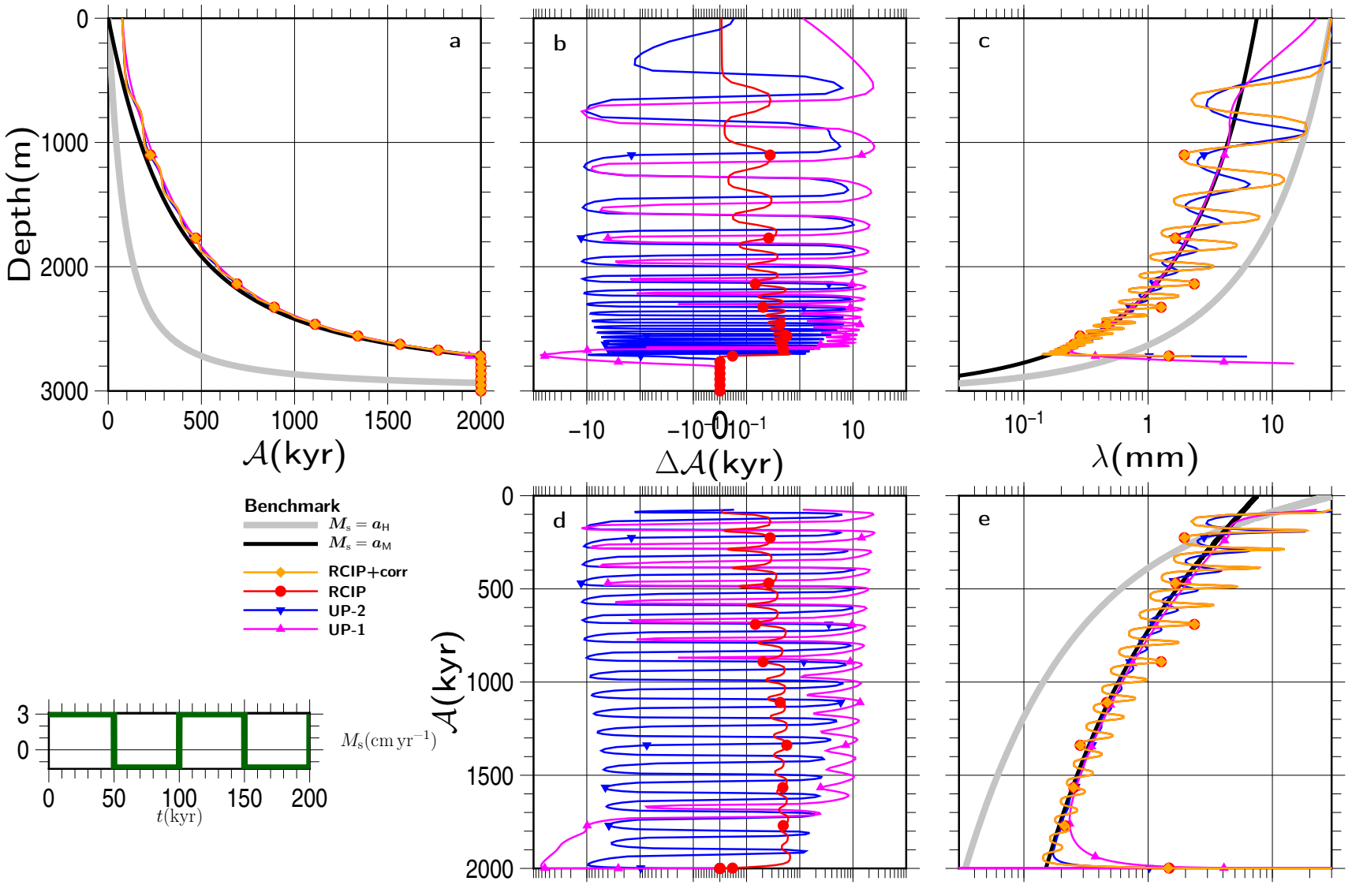

Figure S133
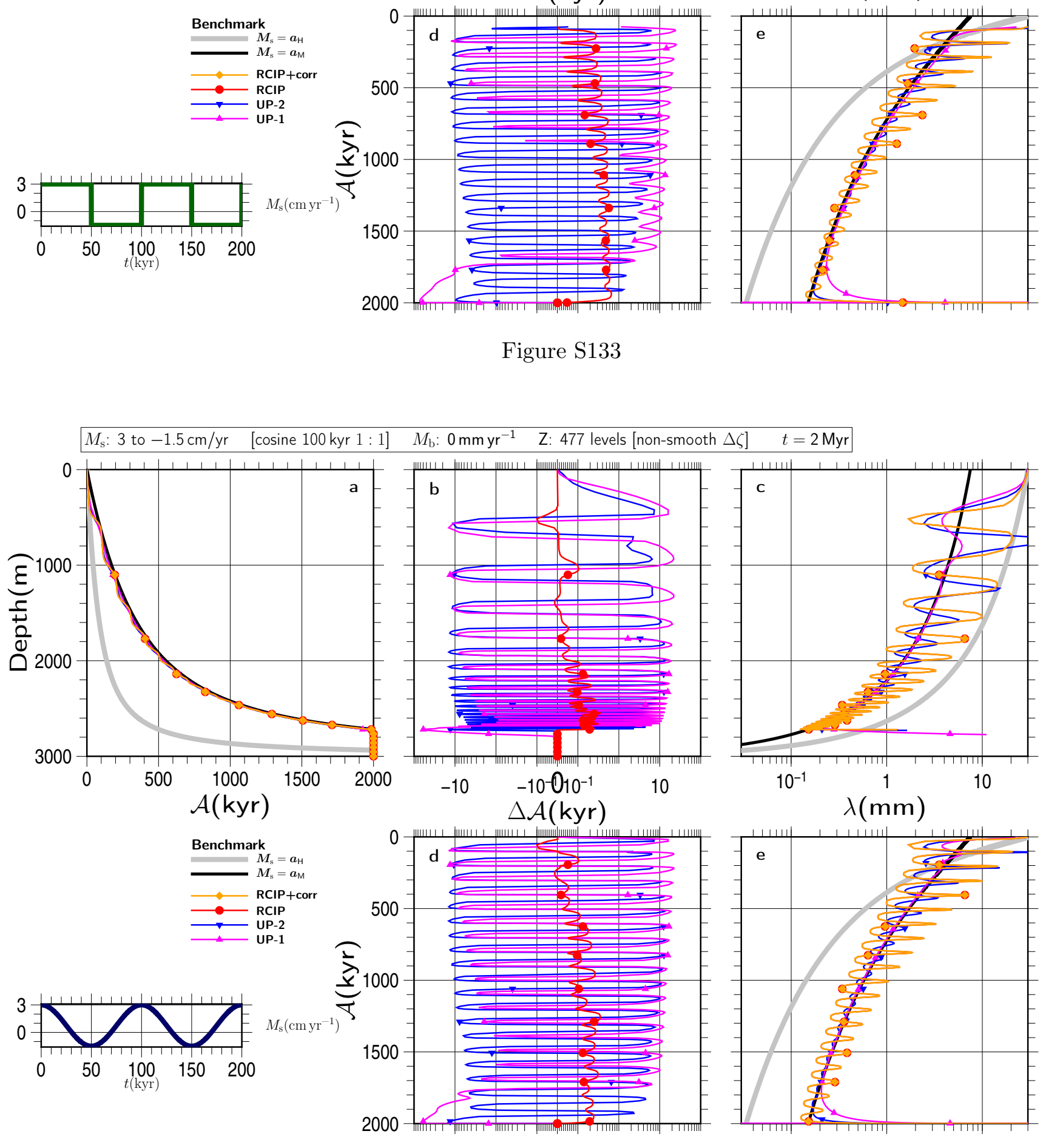

Figure S134 

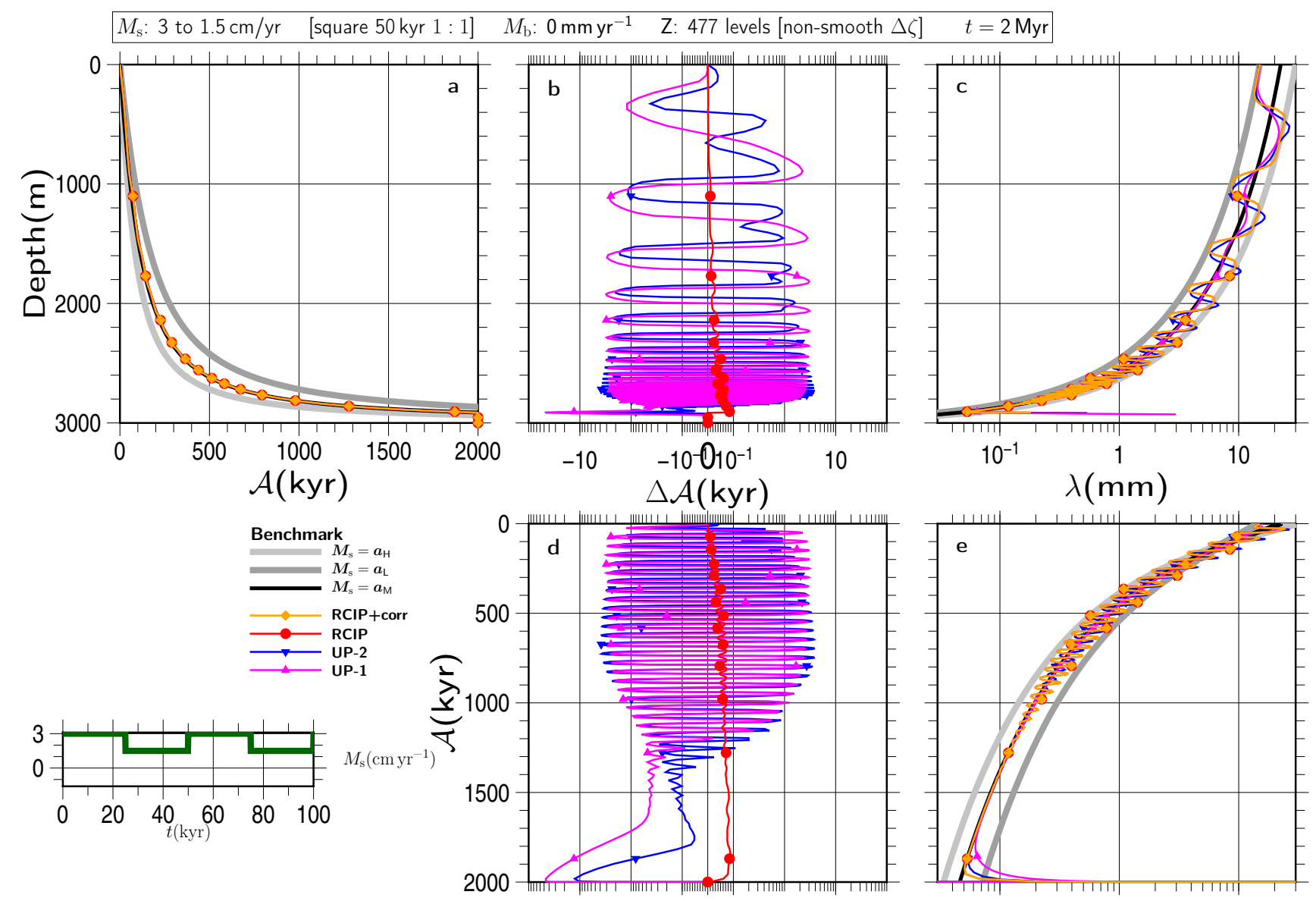

Figure S135
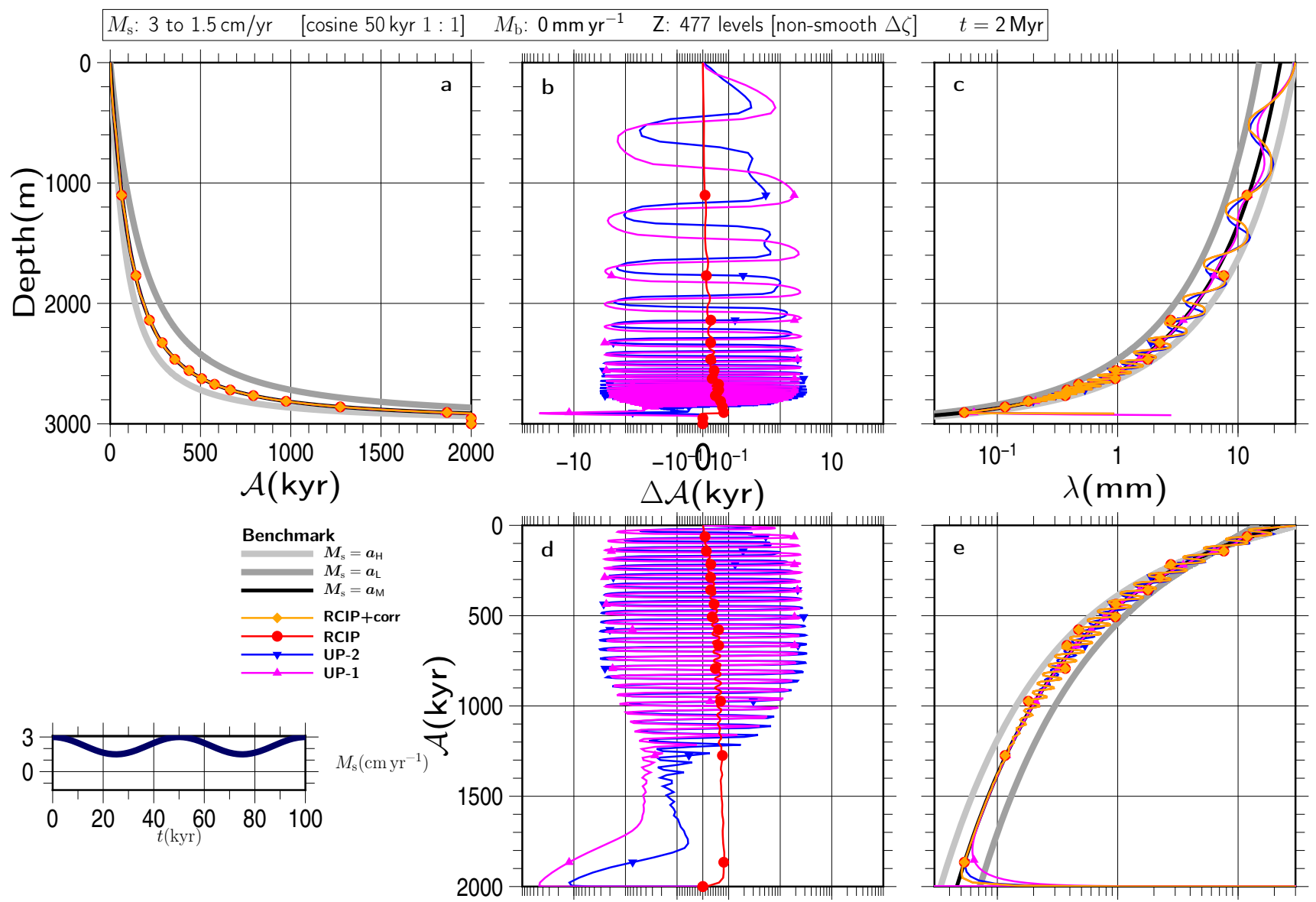

Figure S136 

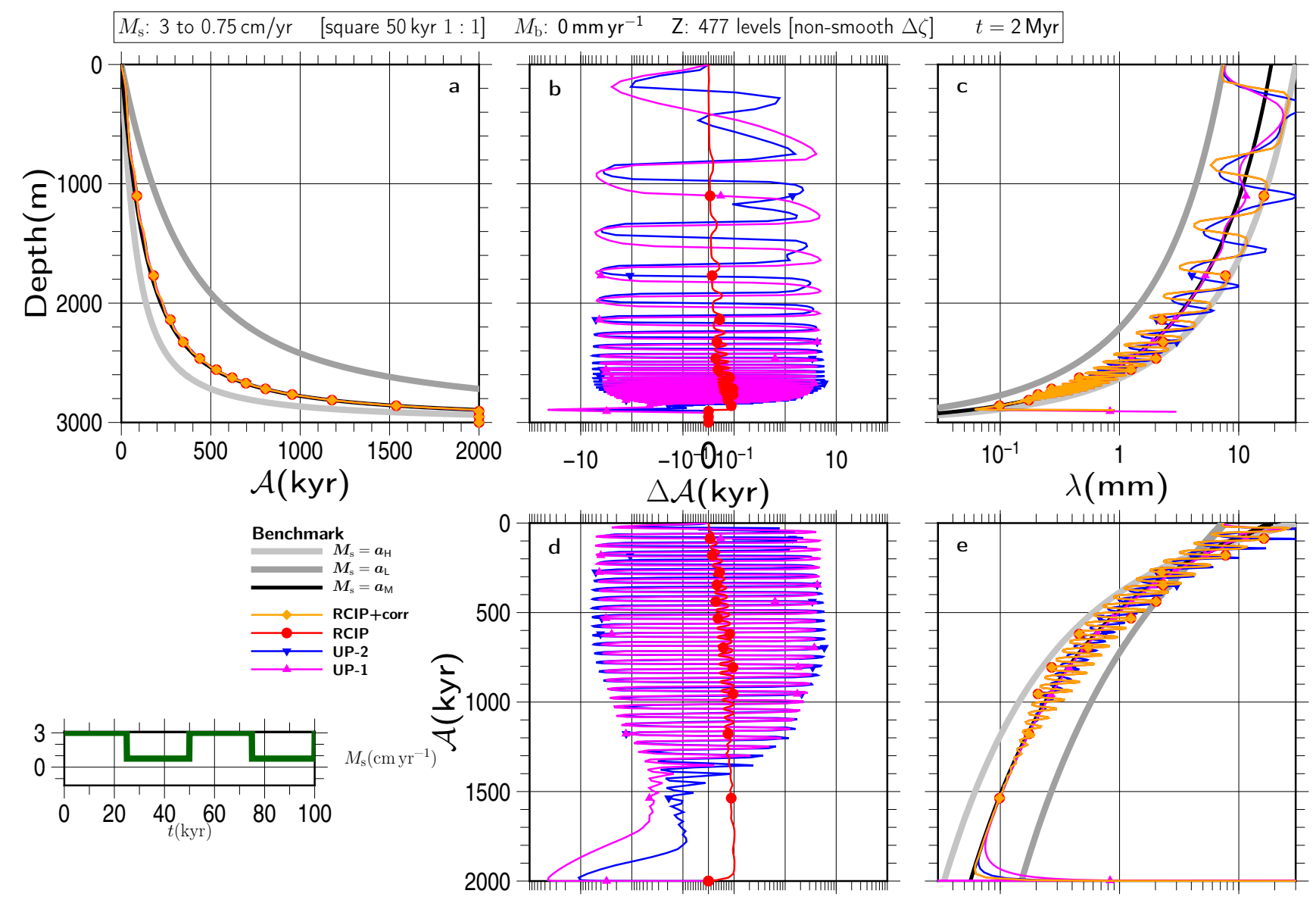

Figure S137
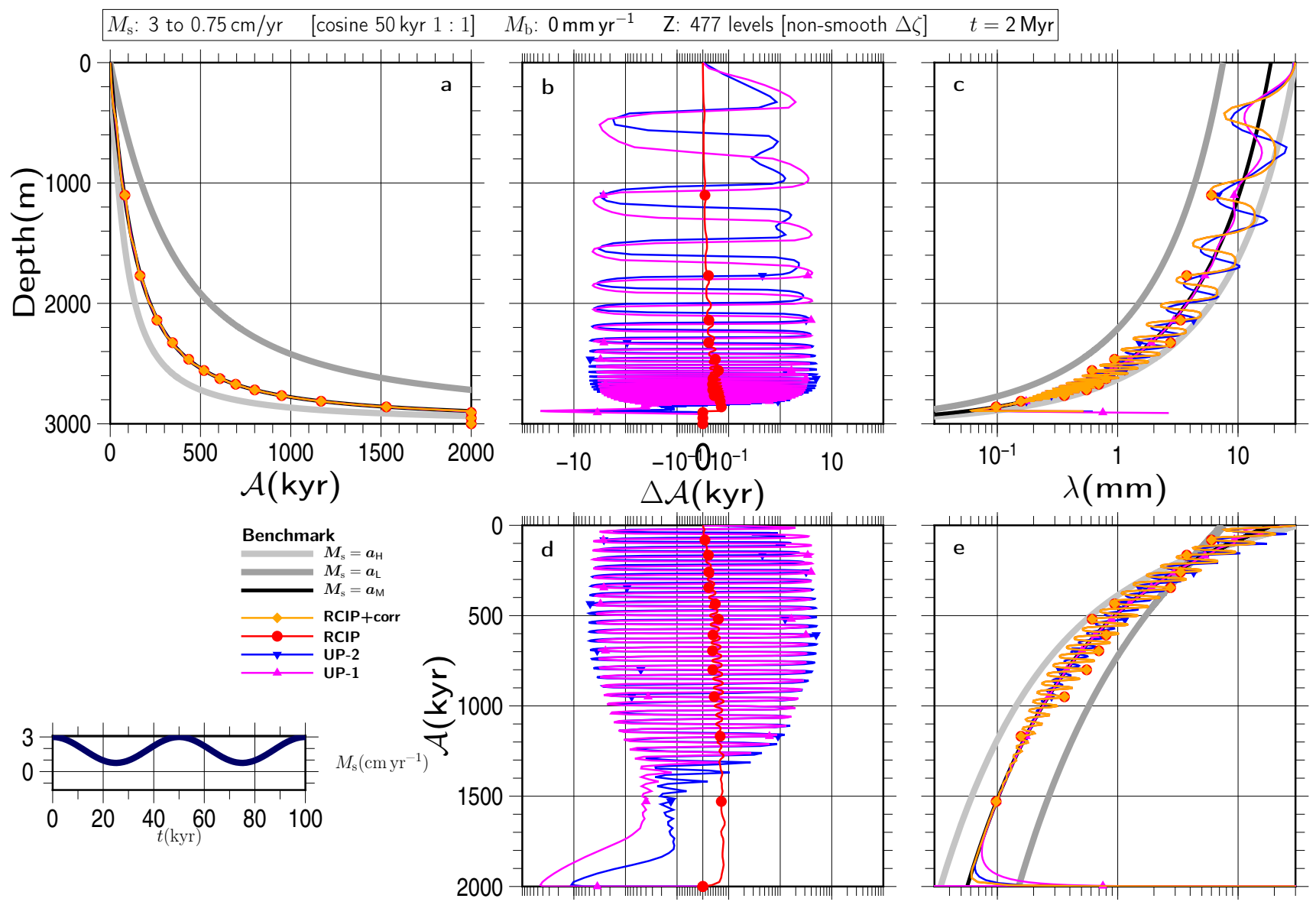

Figure S138 

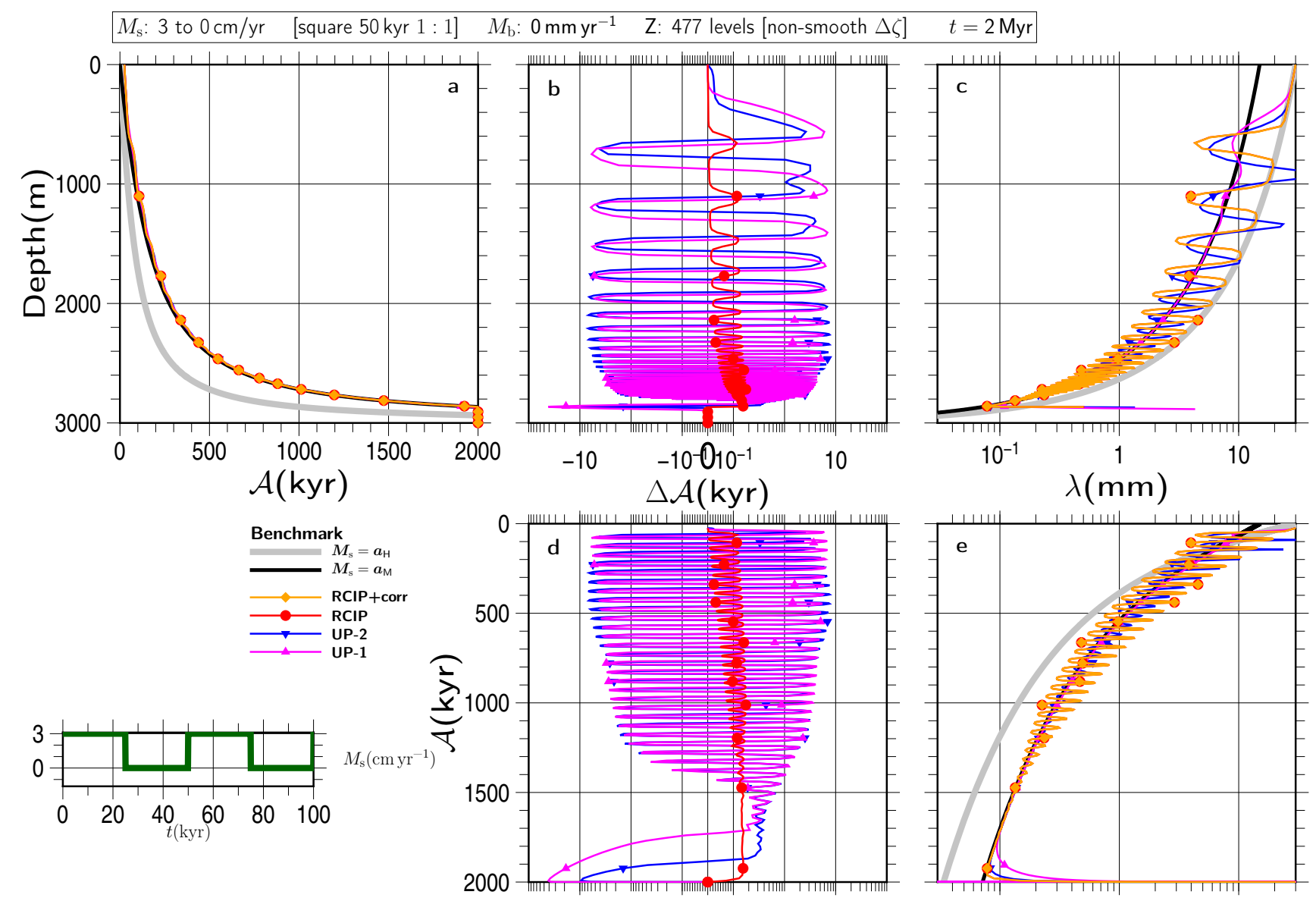

Figure S139
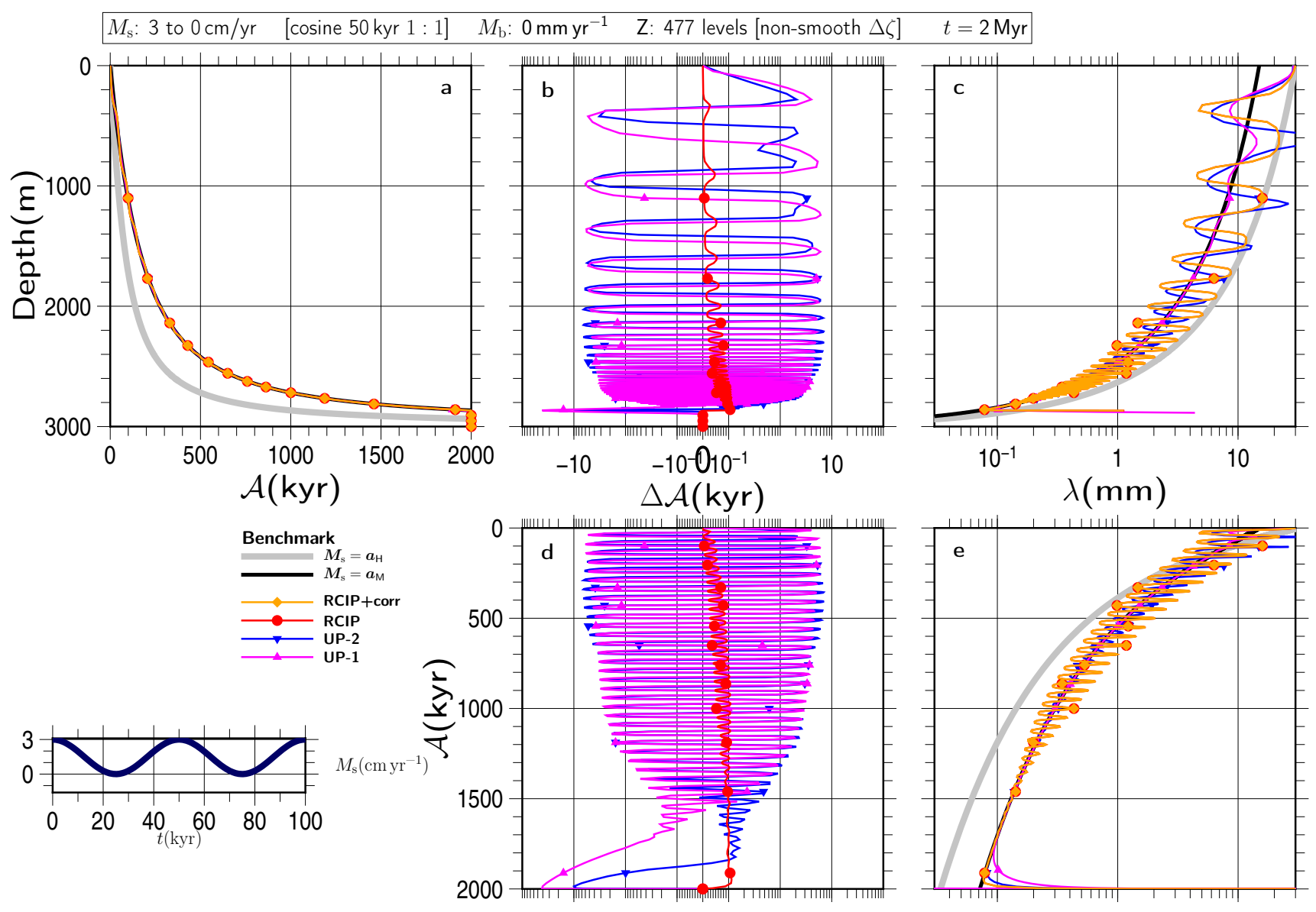

Figure S140 

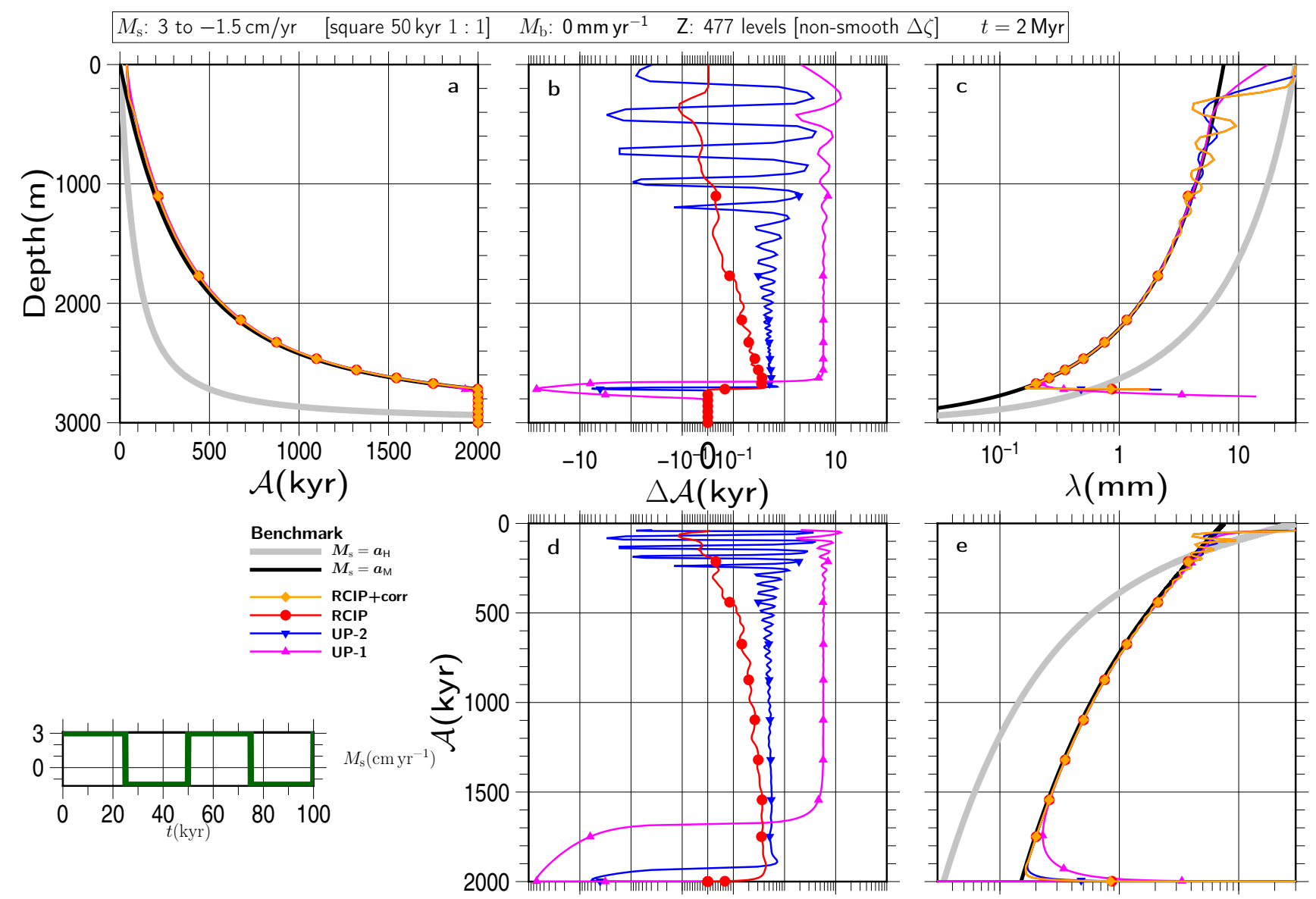

Figure S141
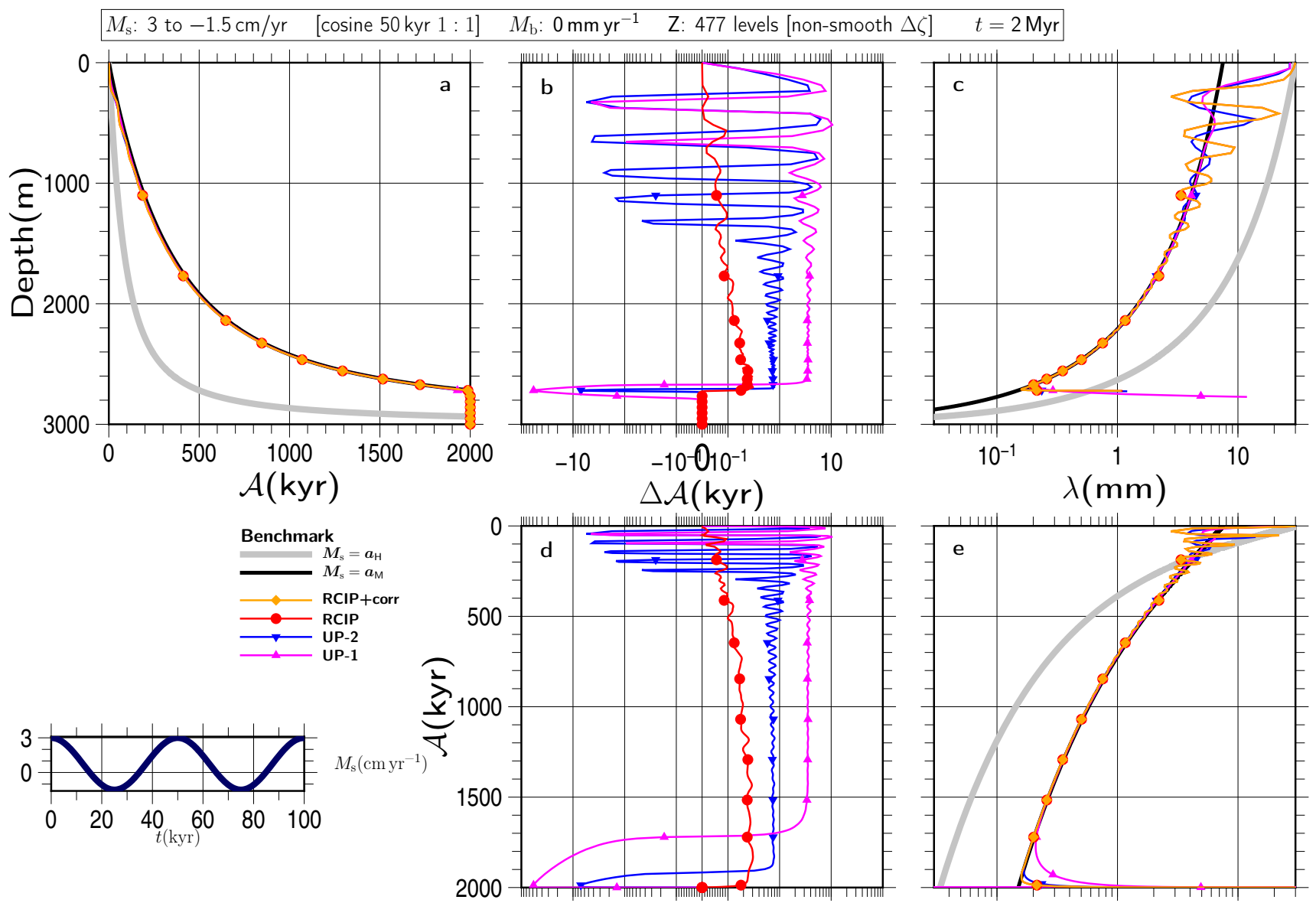

Figure S142 

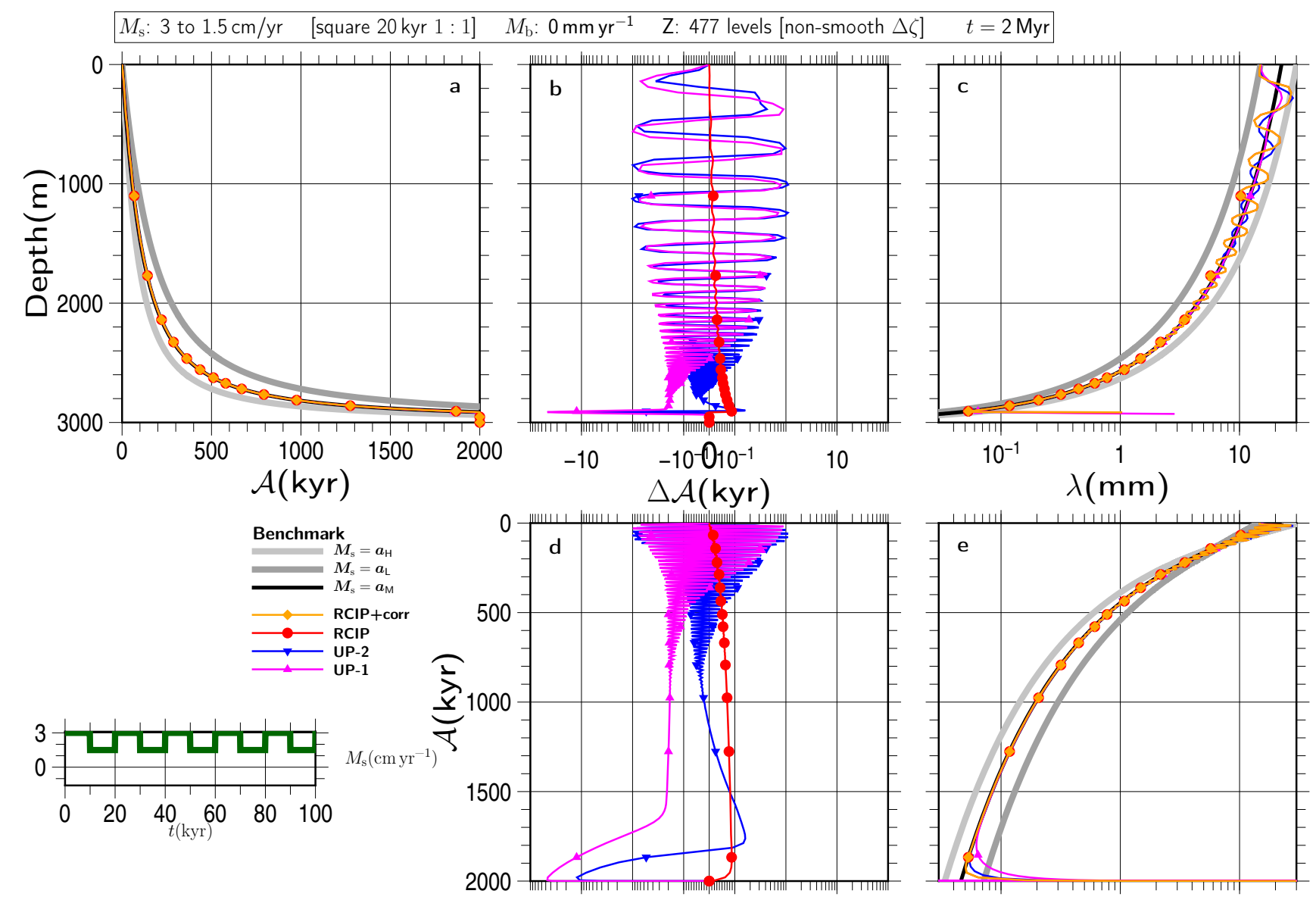

Figure S143
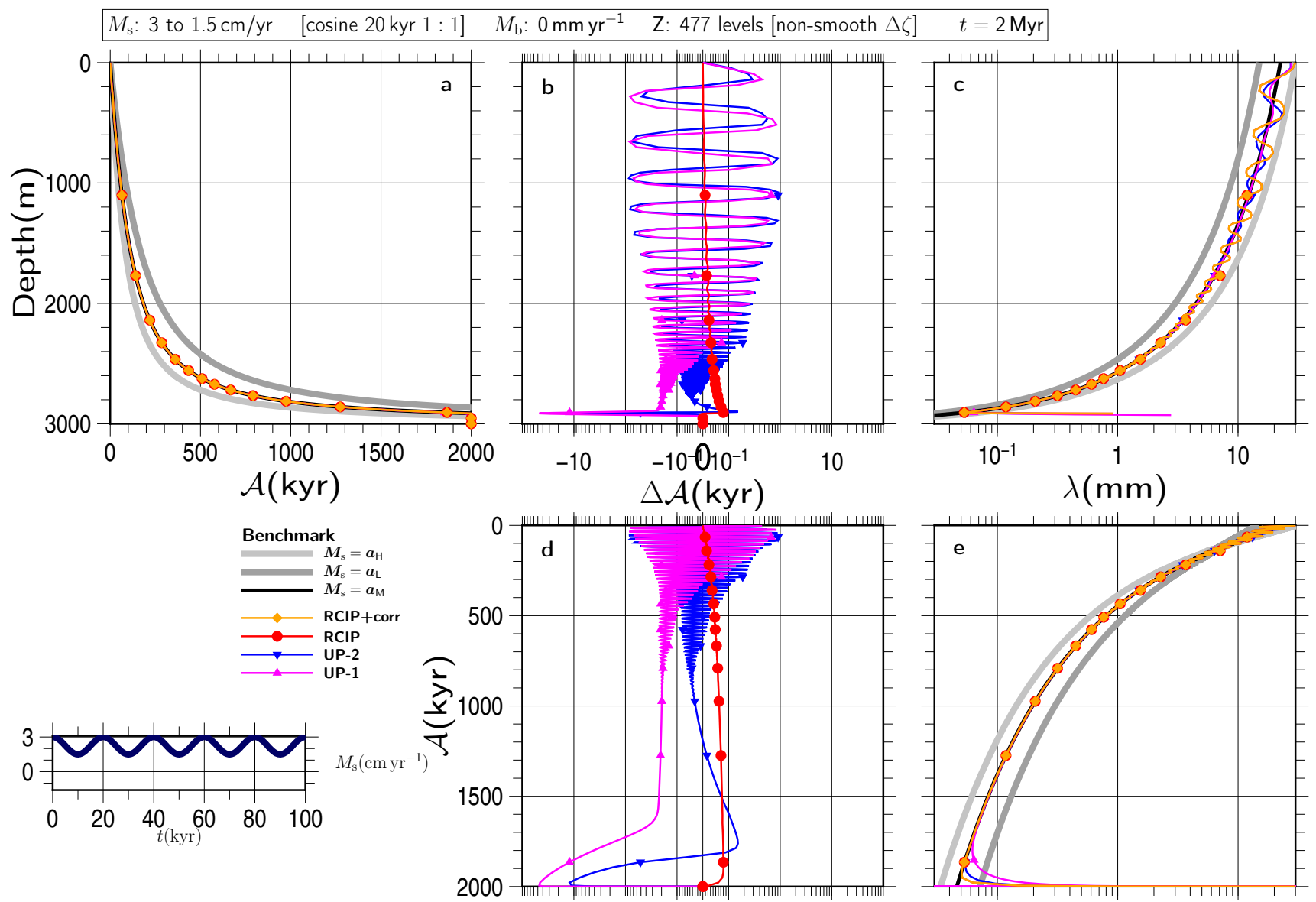

Figure S144 

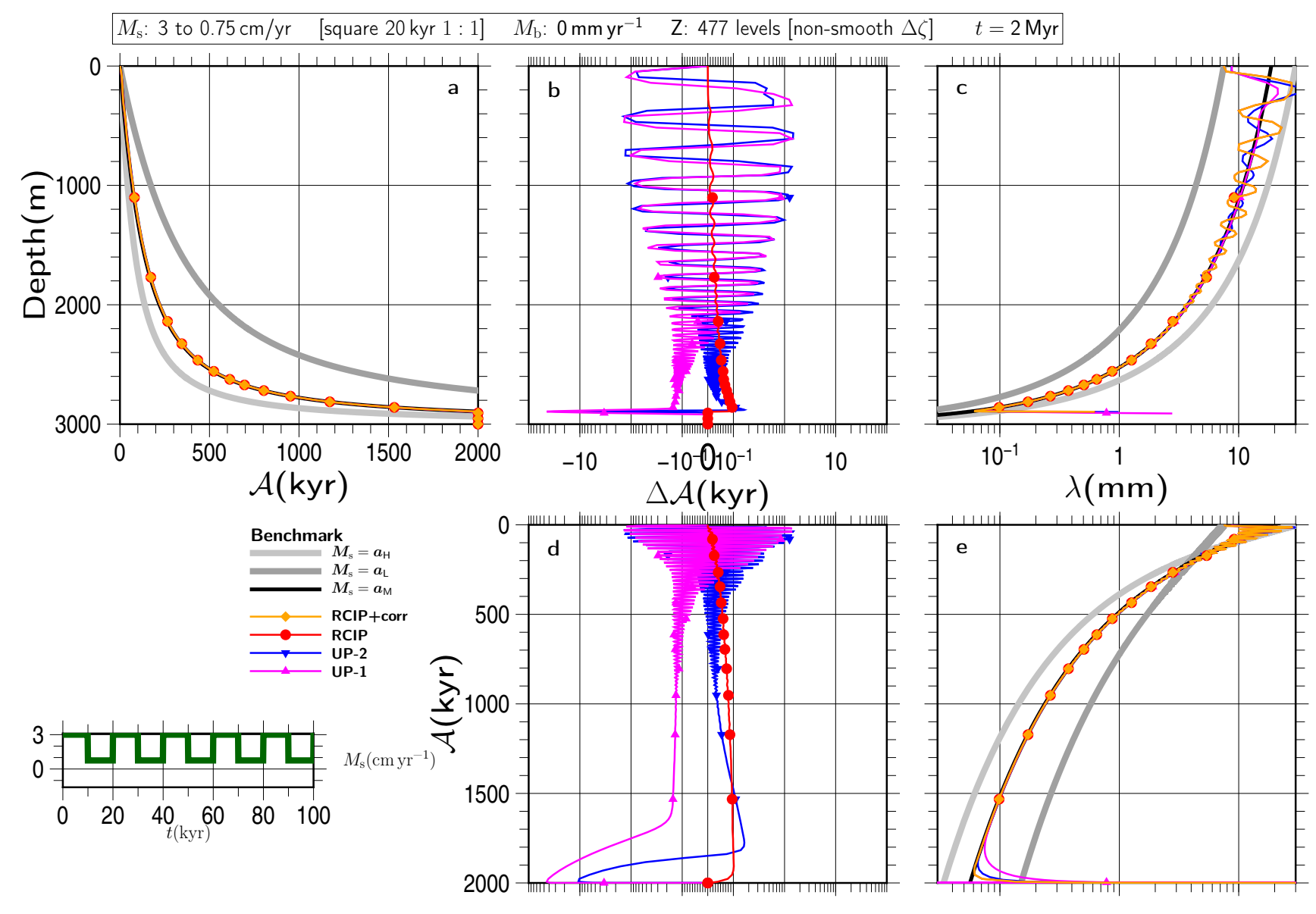

Figure S145
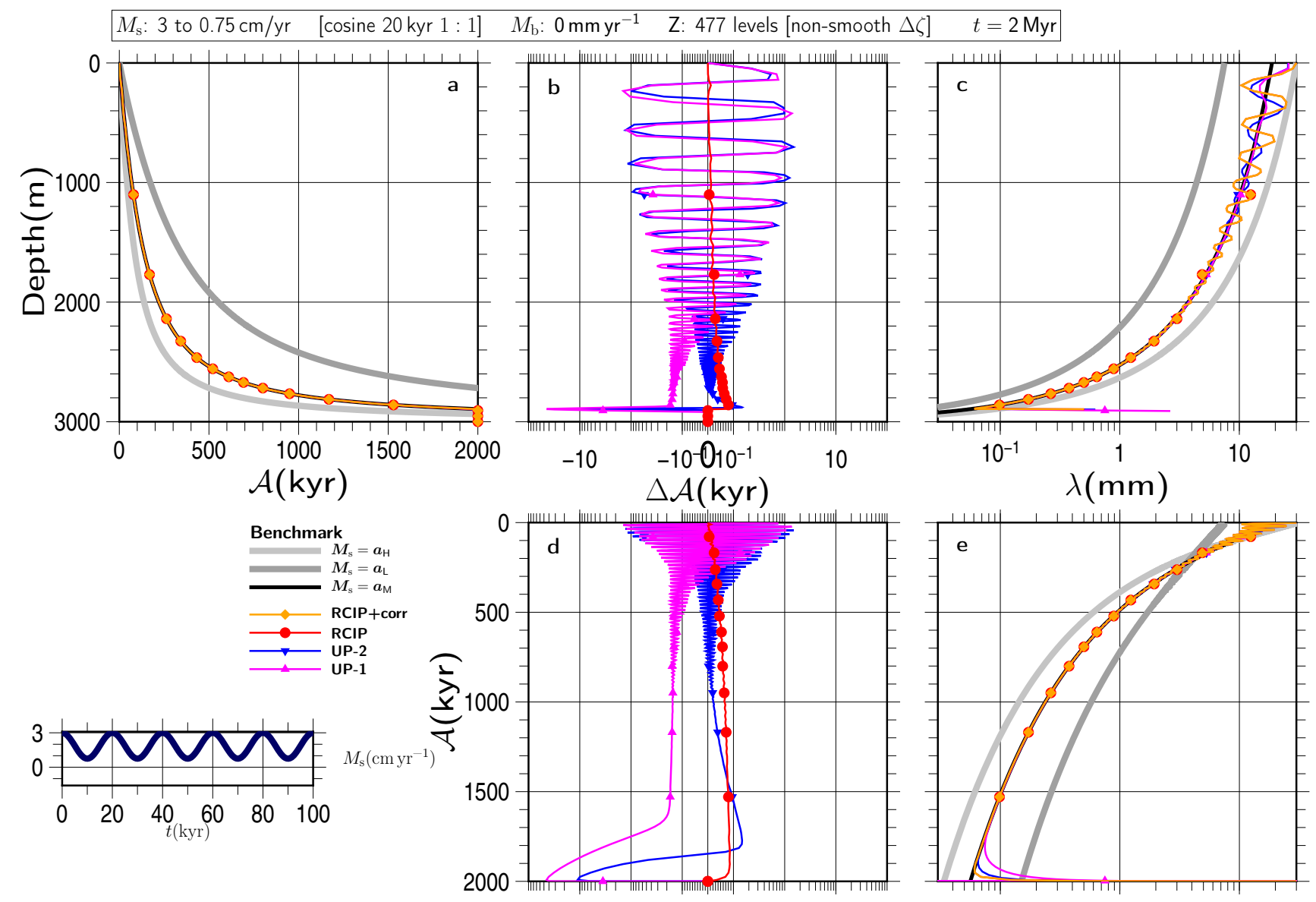

Figure S146 

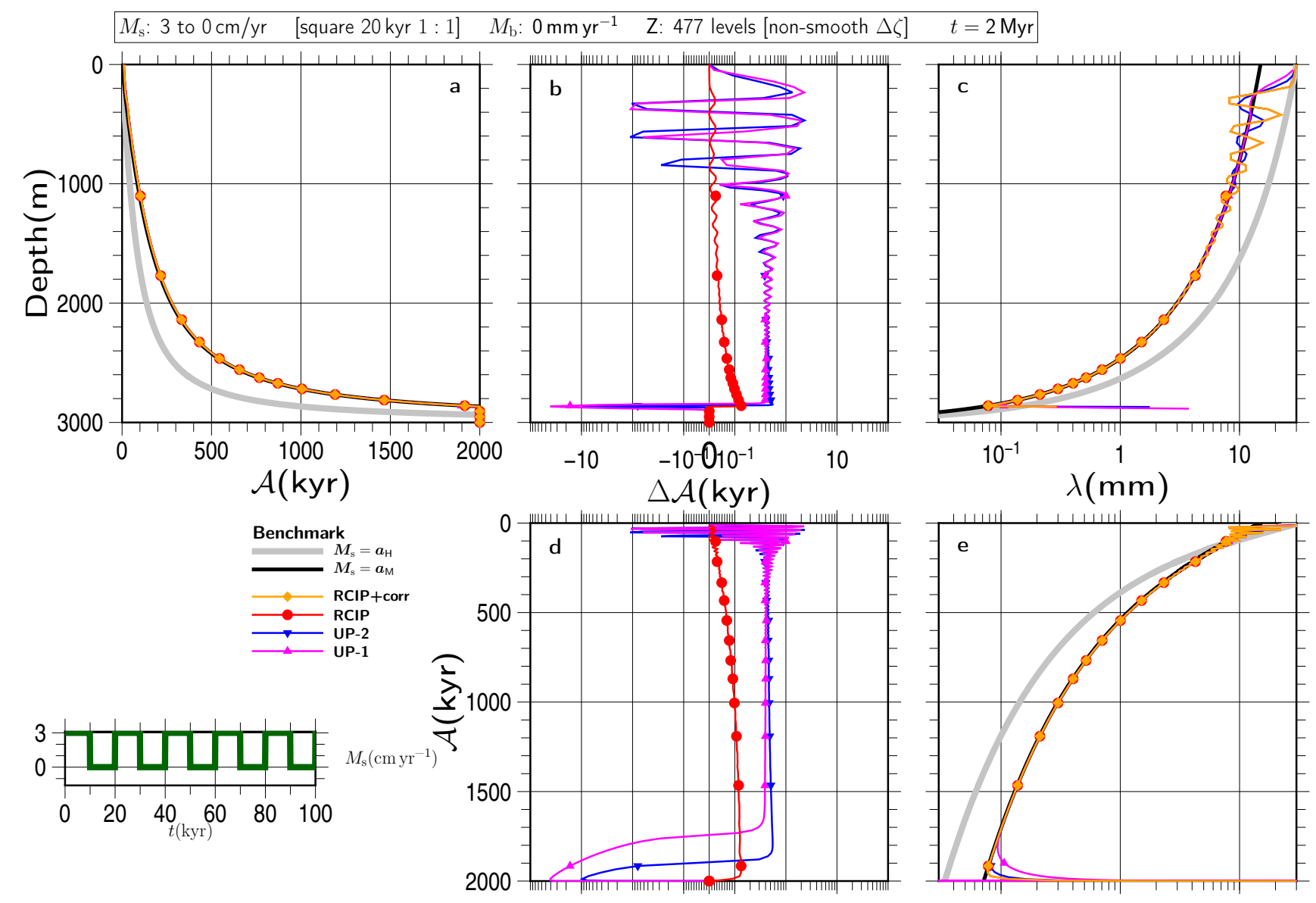

Figure S147
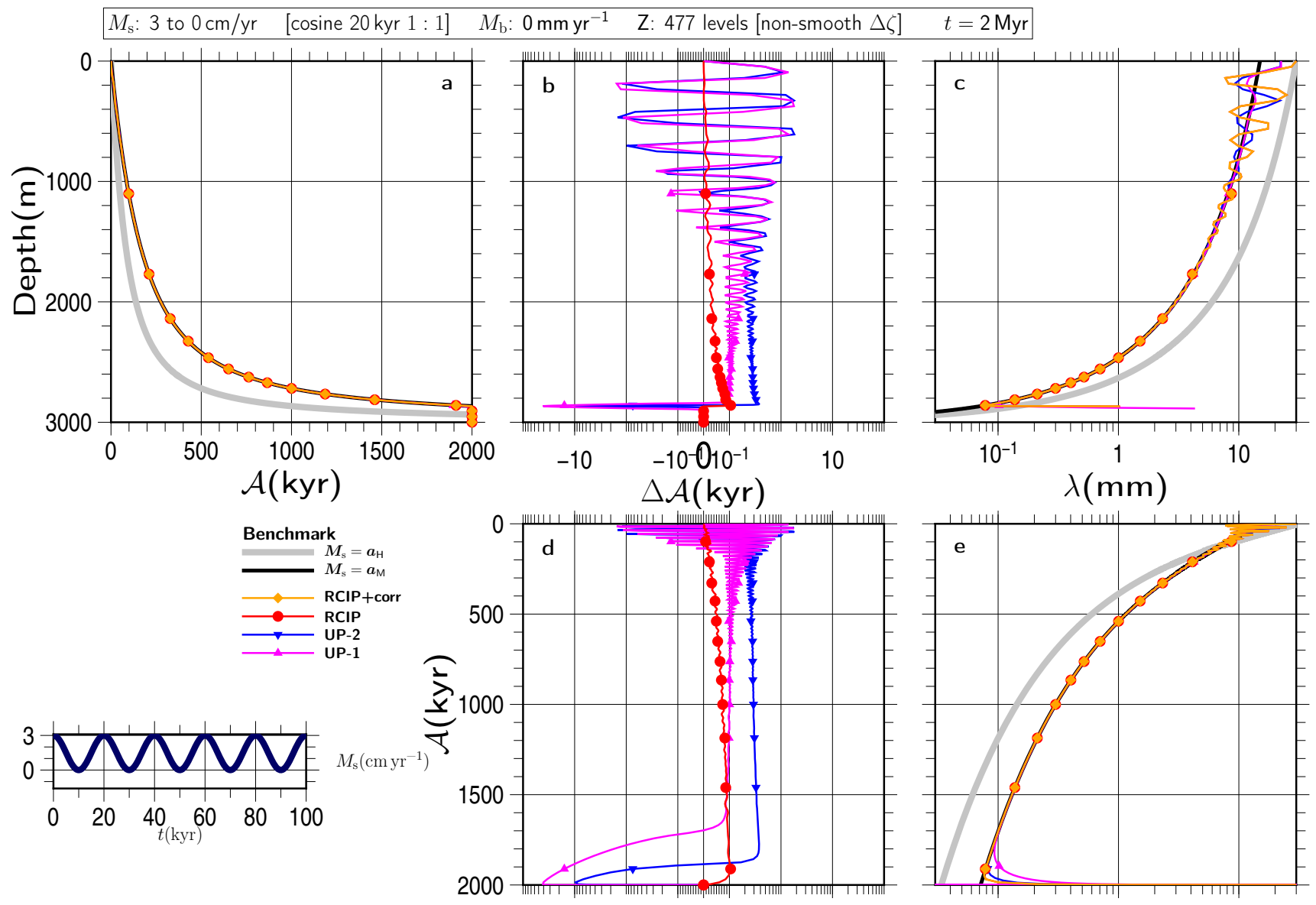

Figure S148 


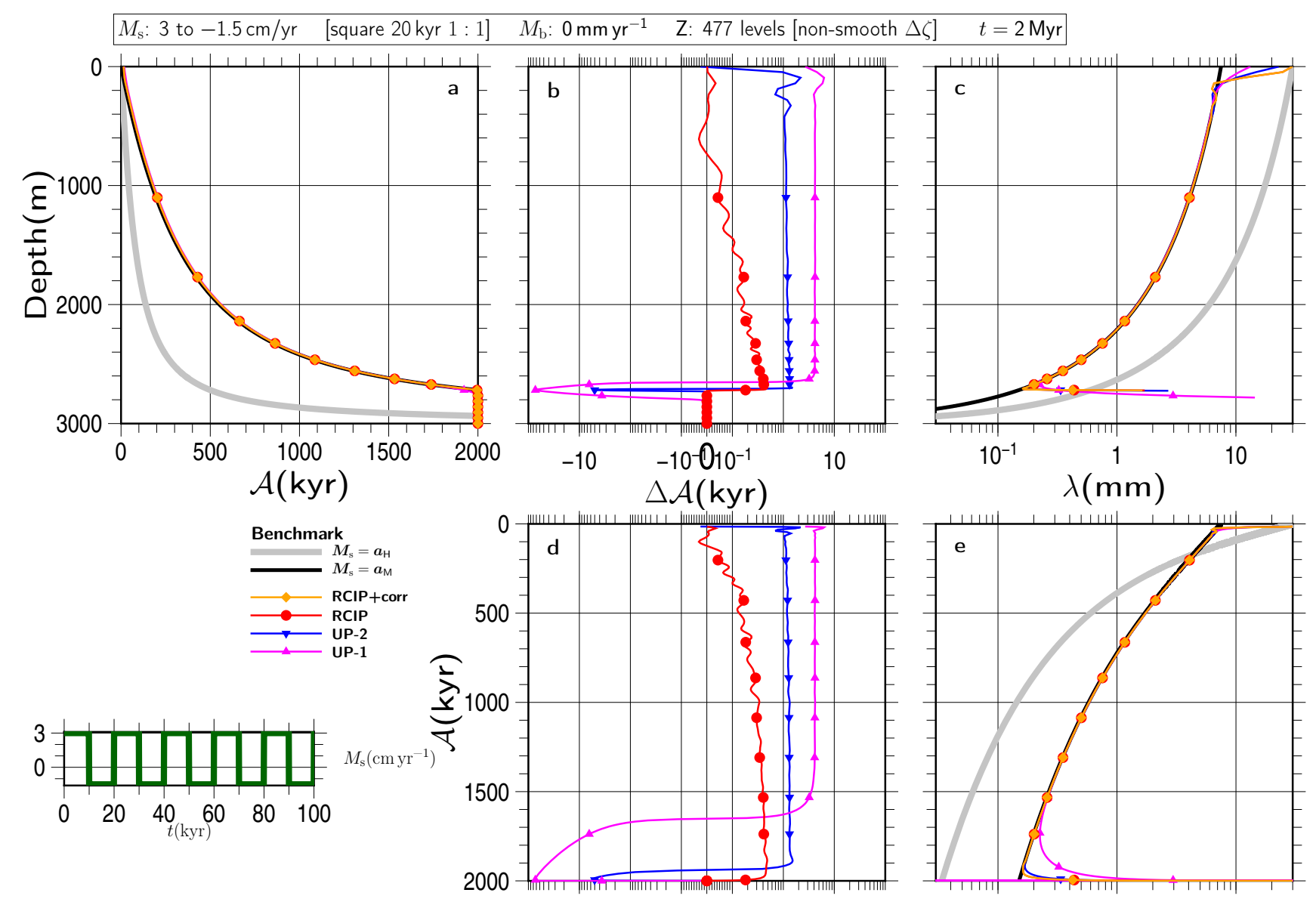

Figure S149

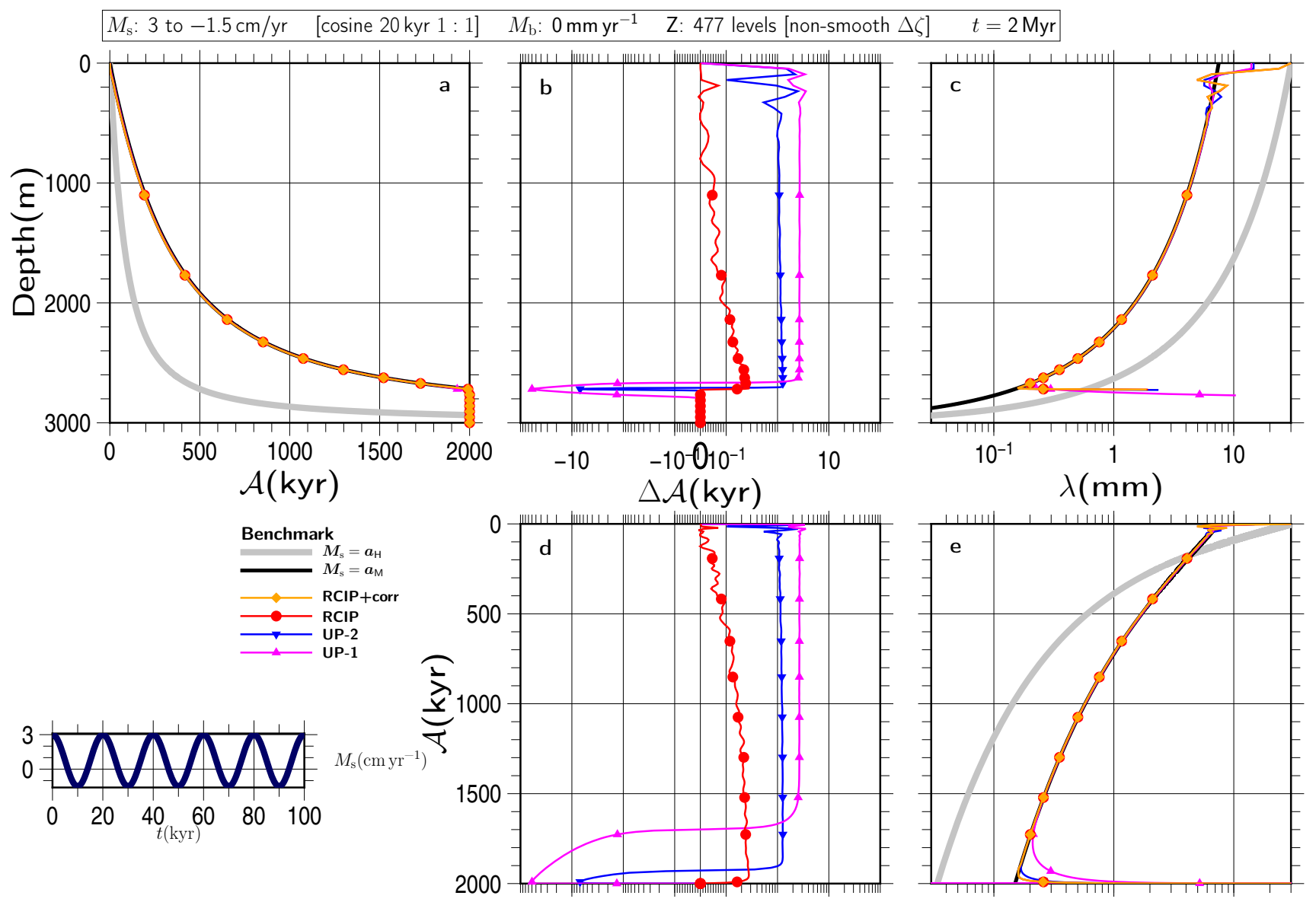

Figure S150 


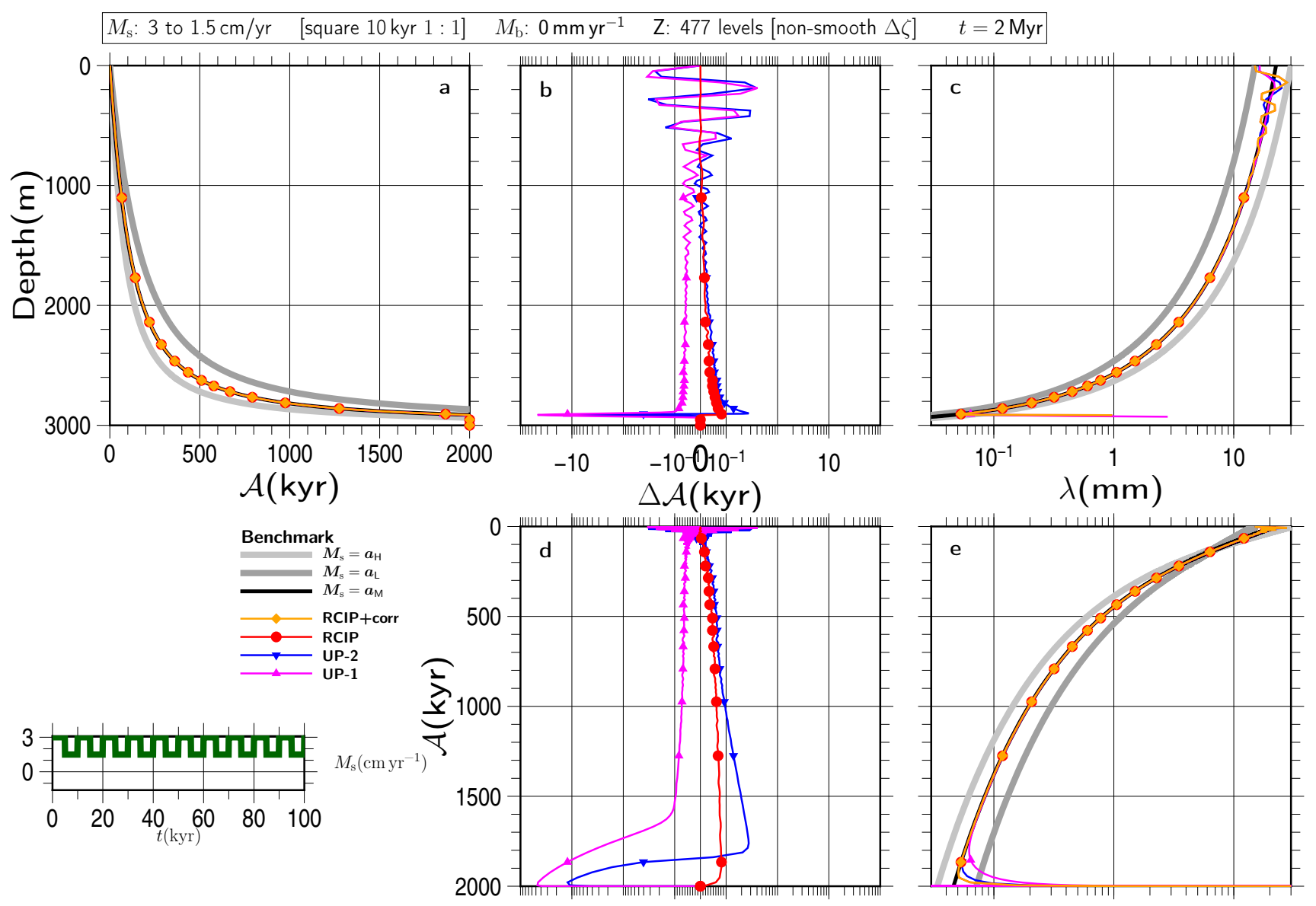

Figure S151

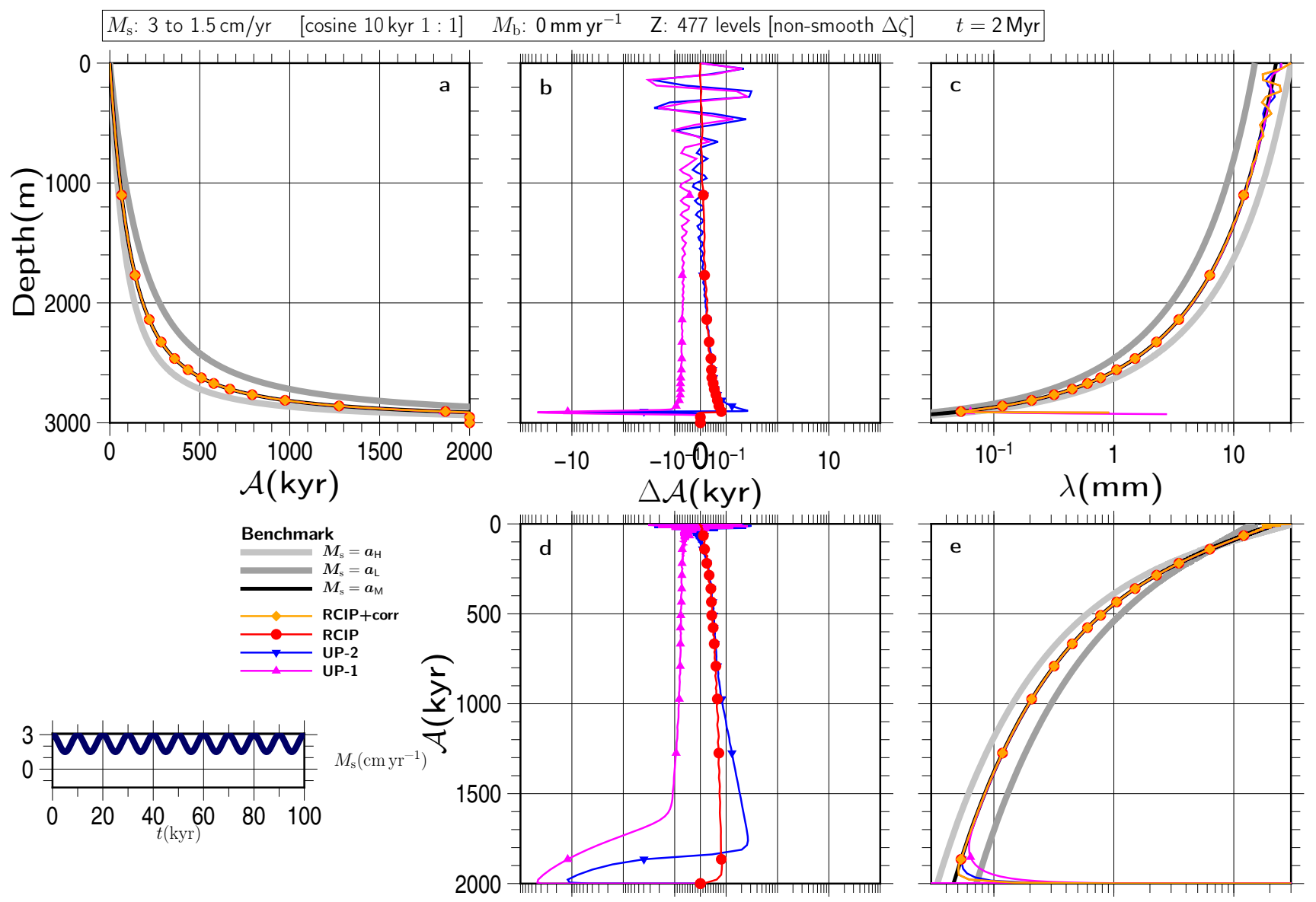

Figure S152 

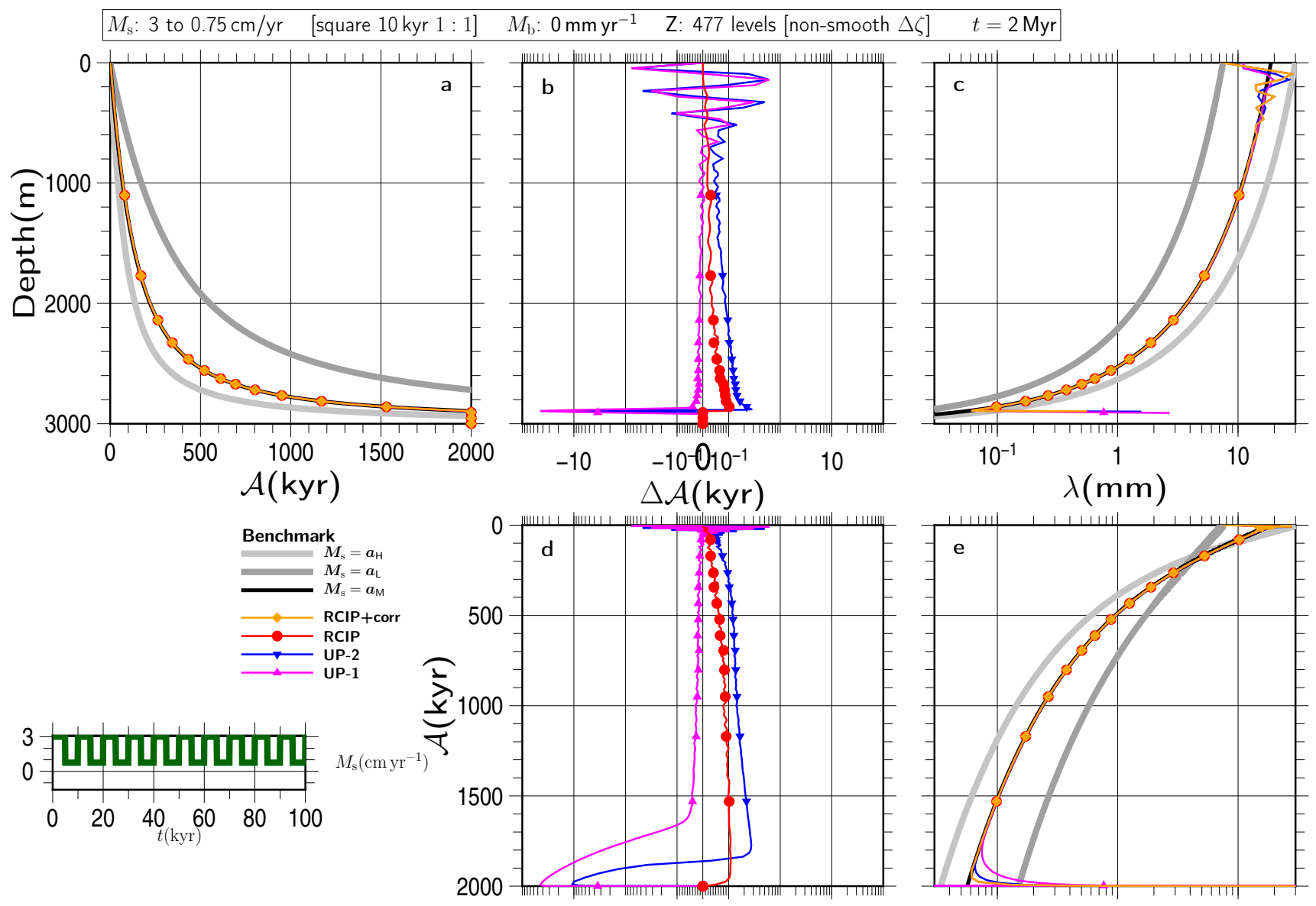

Figure S153

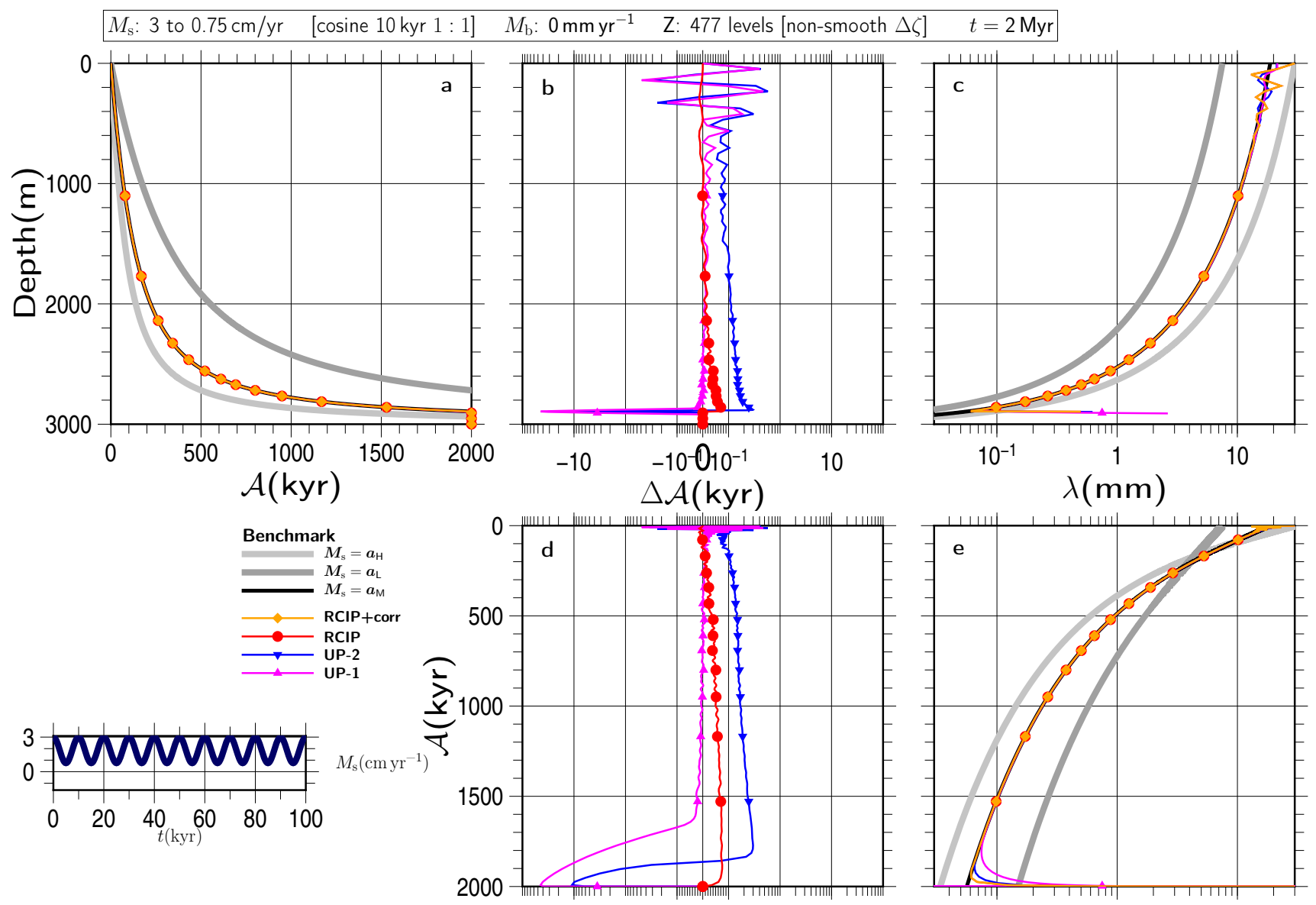

Figure S154 

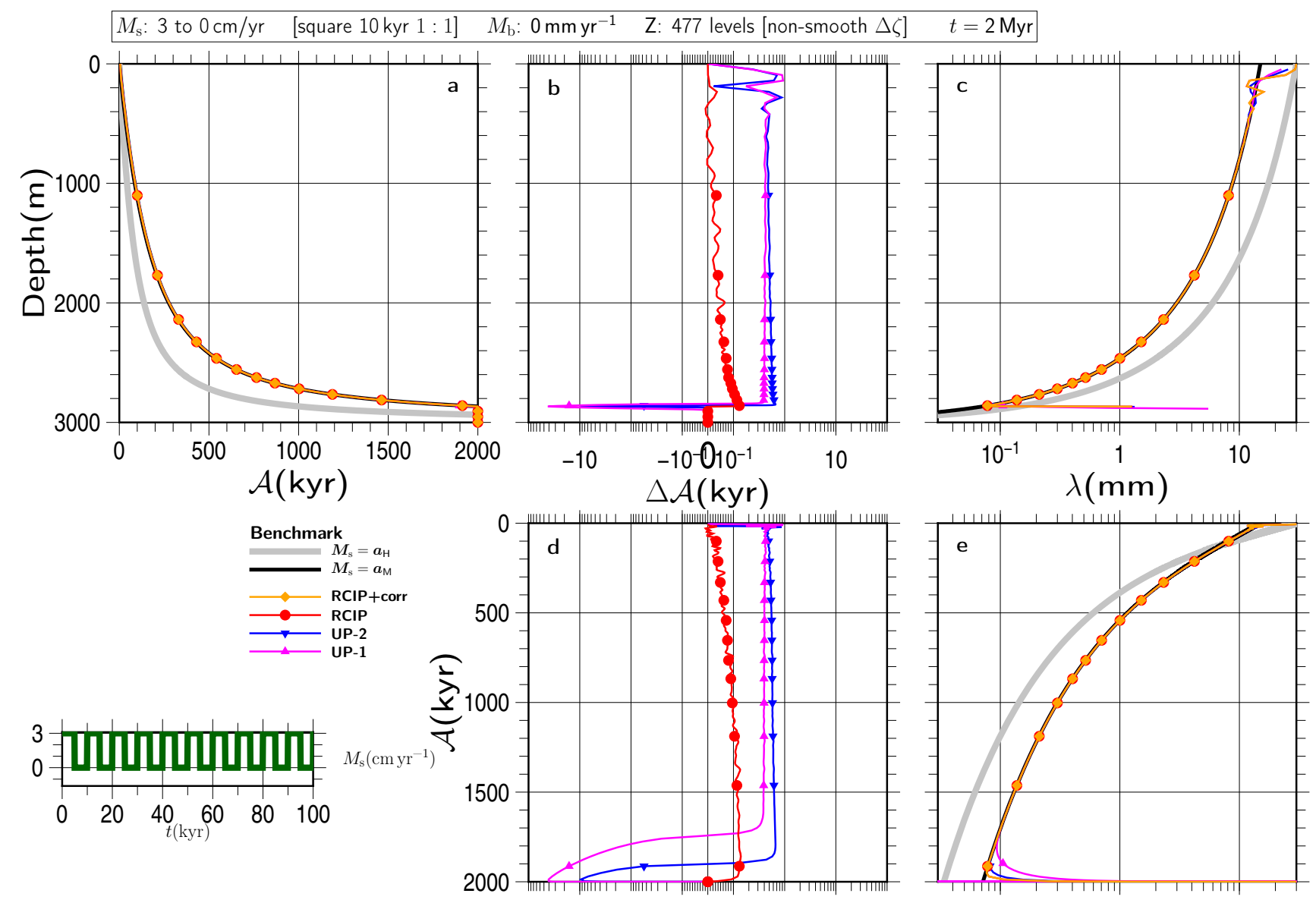

Figure S155
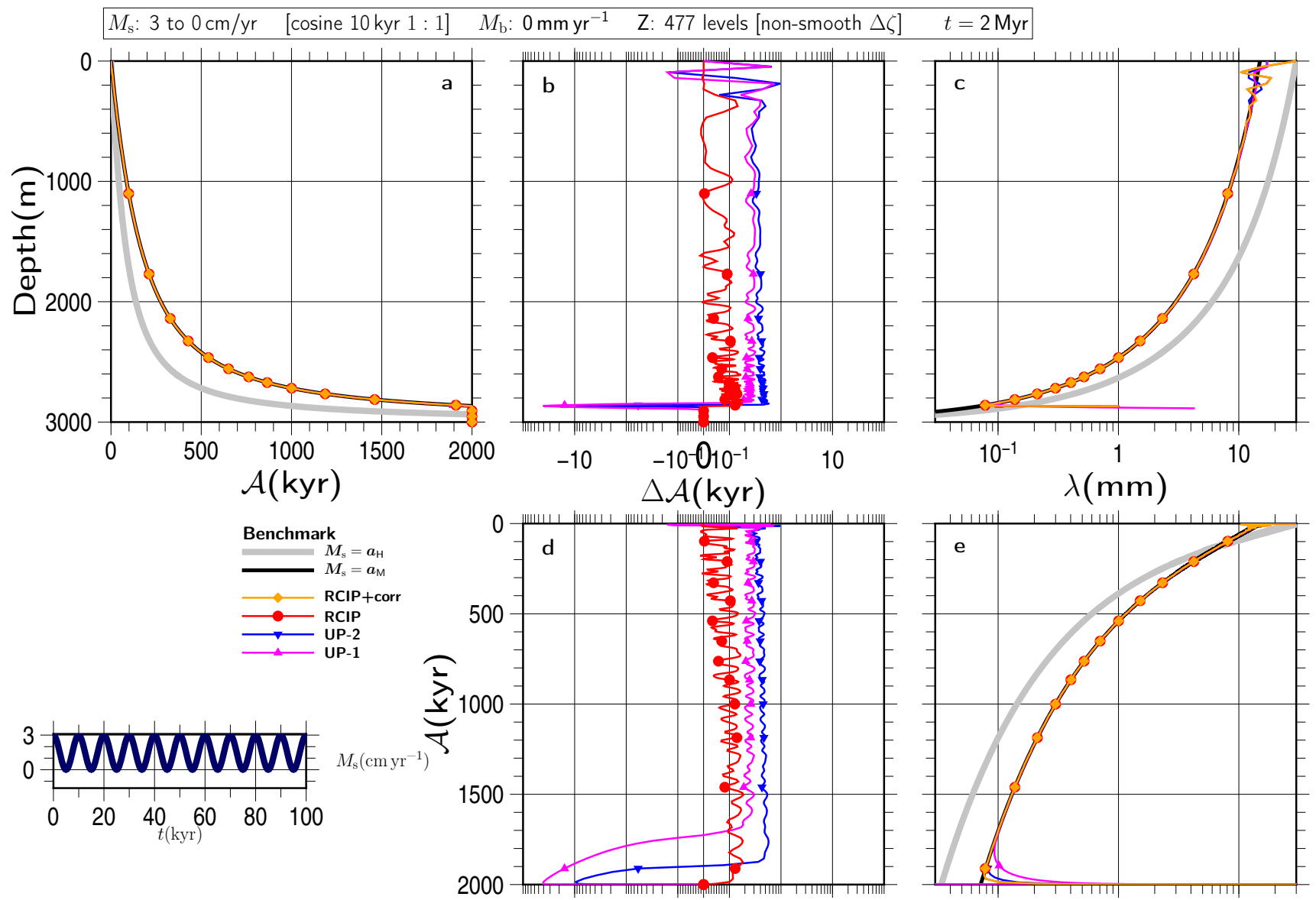

Figure S156 


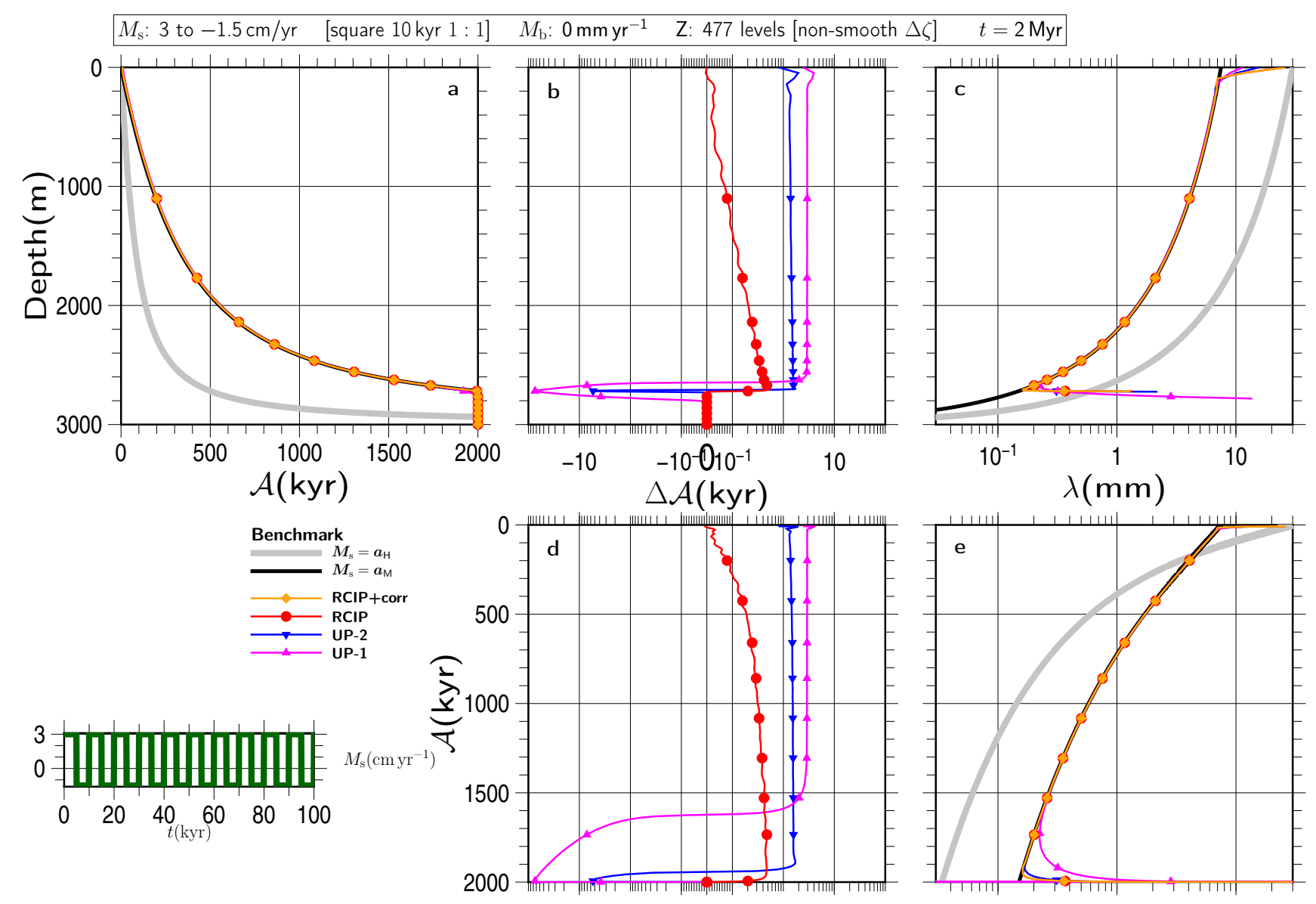

Figure S157

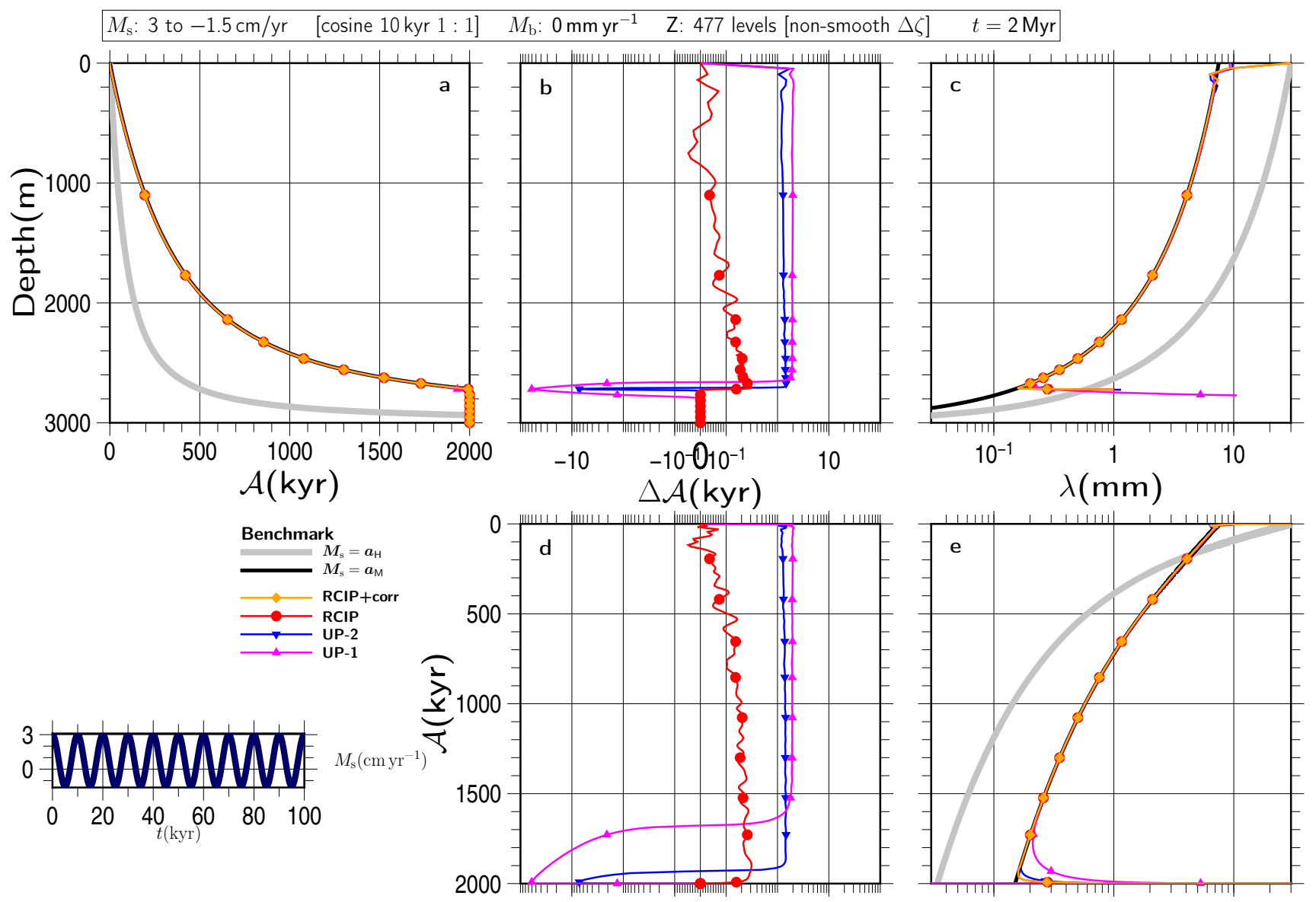

Figure S158 

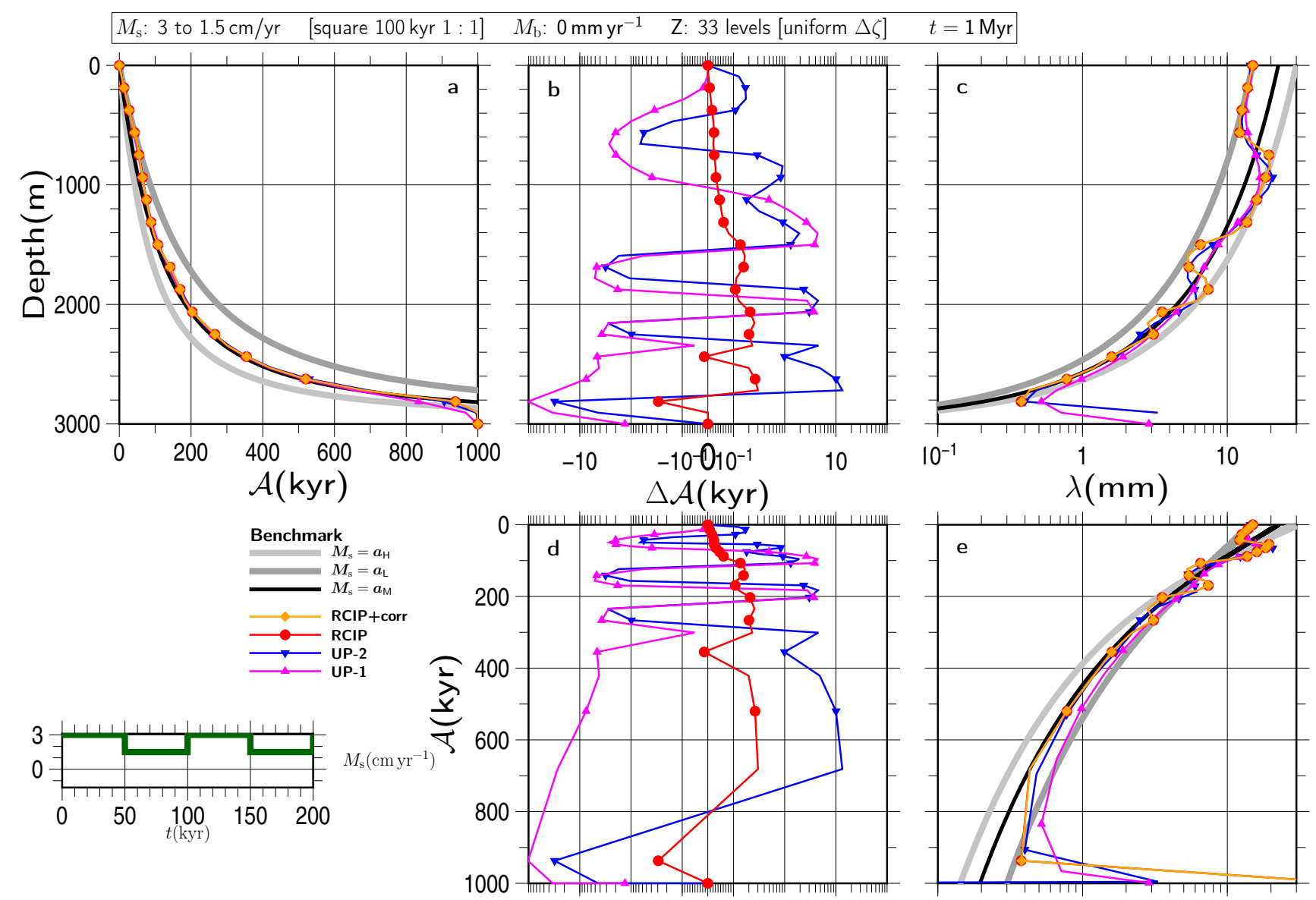

Figure S159
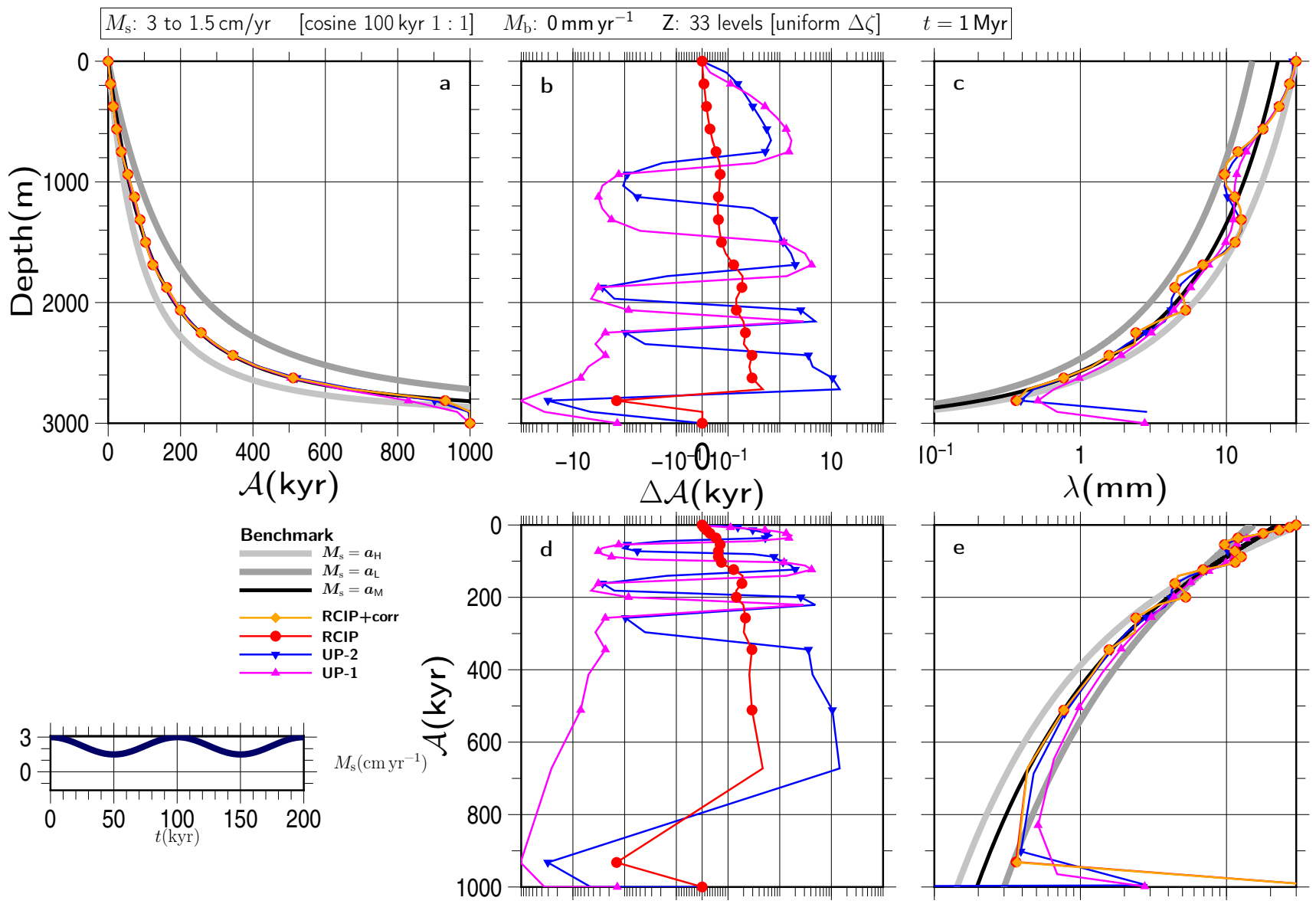

Figure S160 

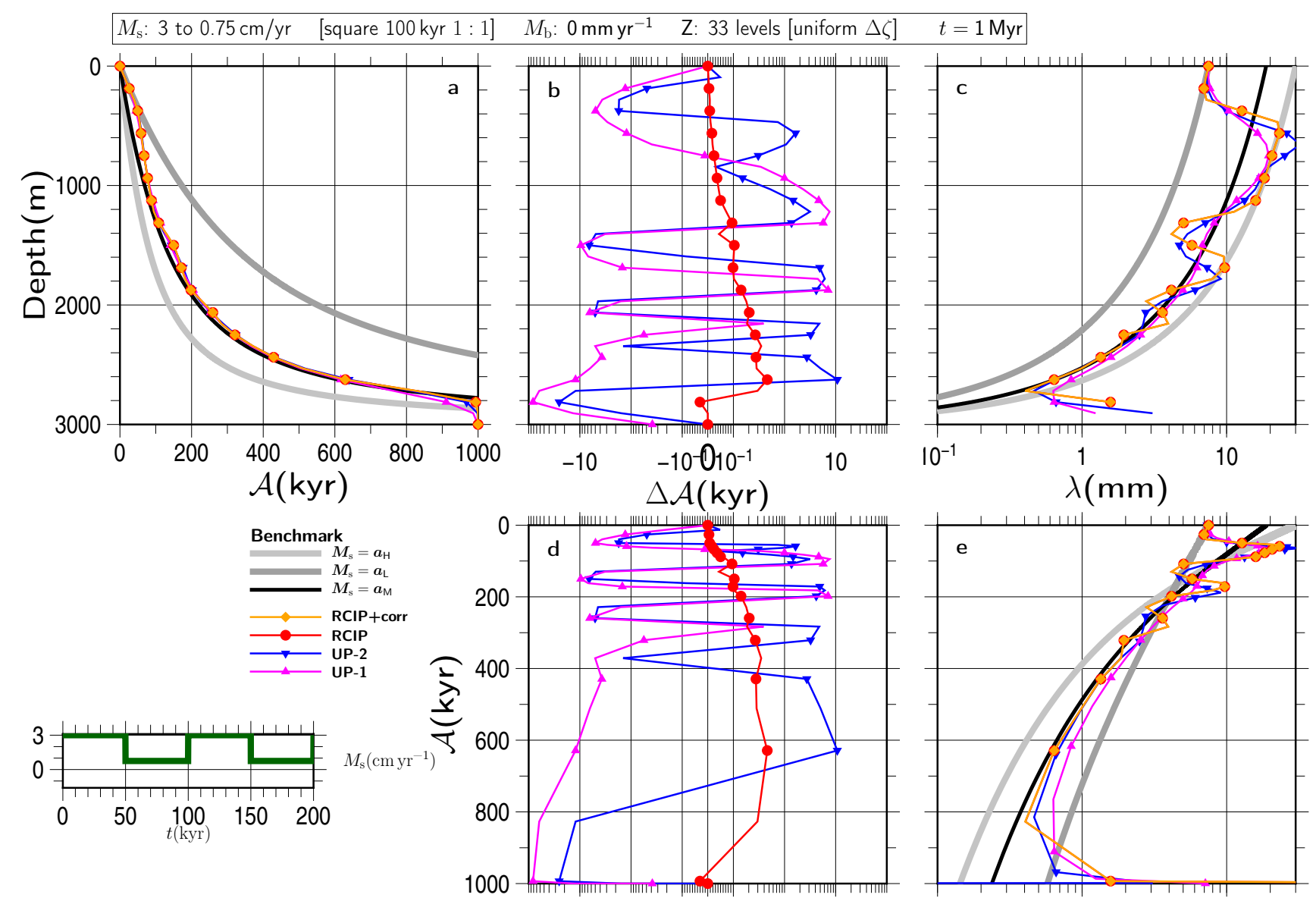

Figure S161
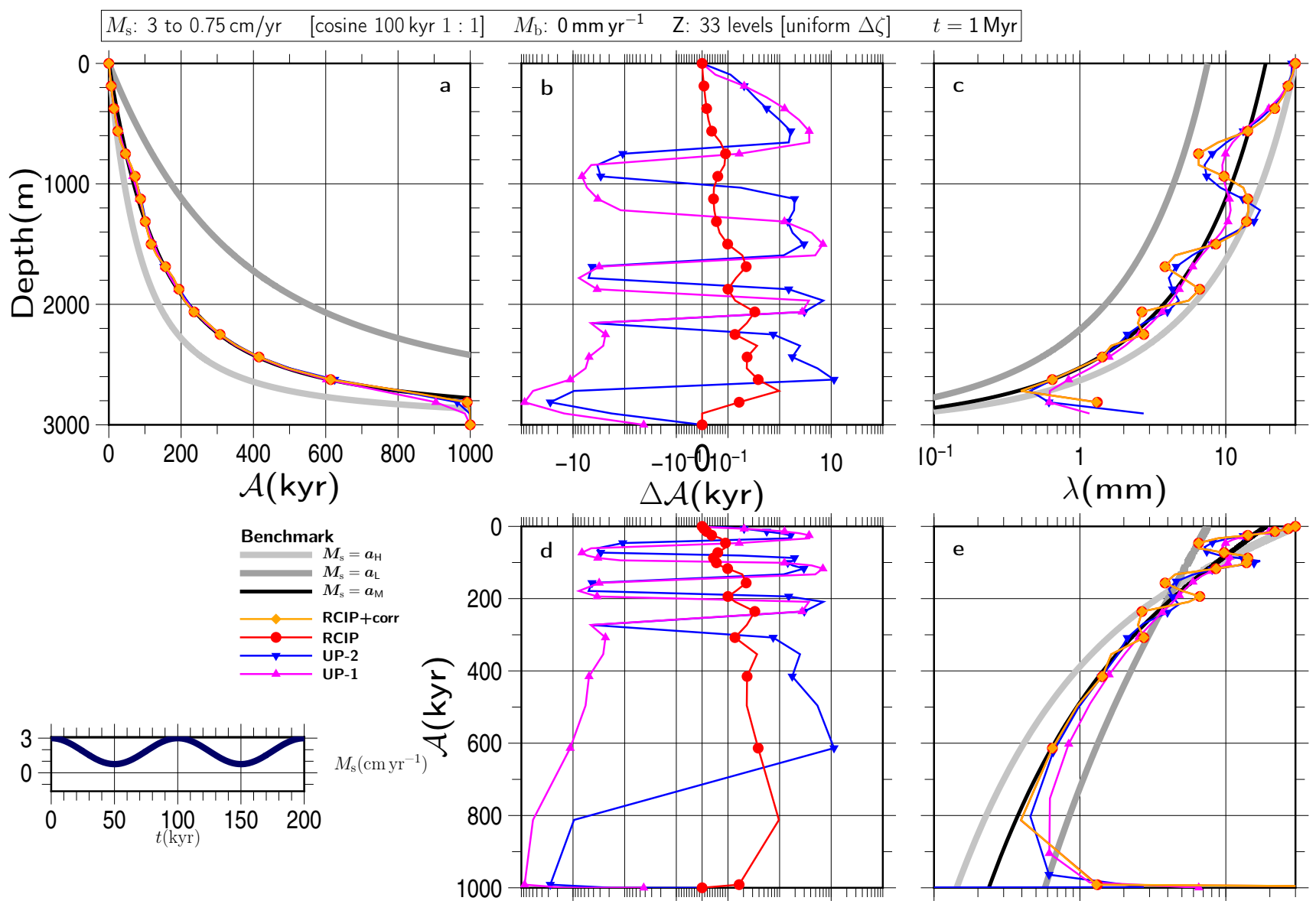

Figure S162 

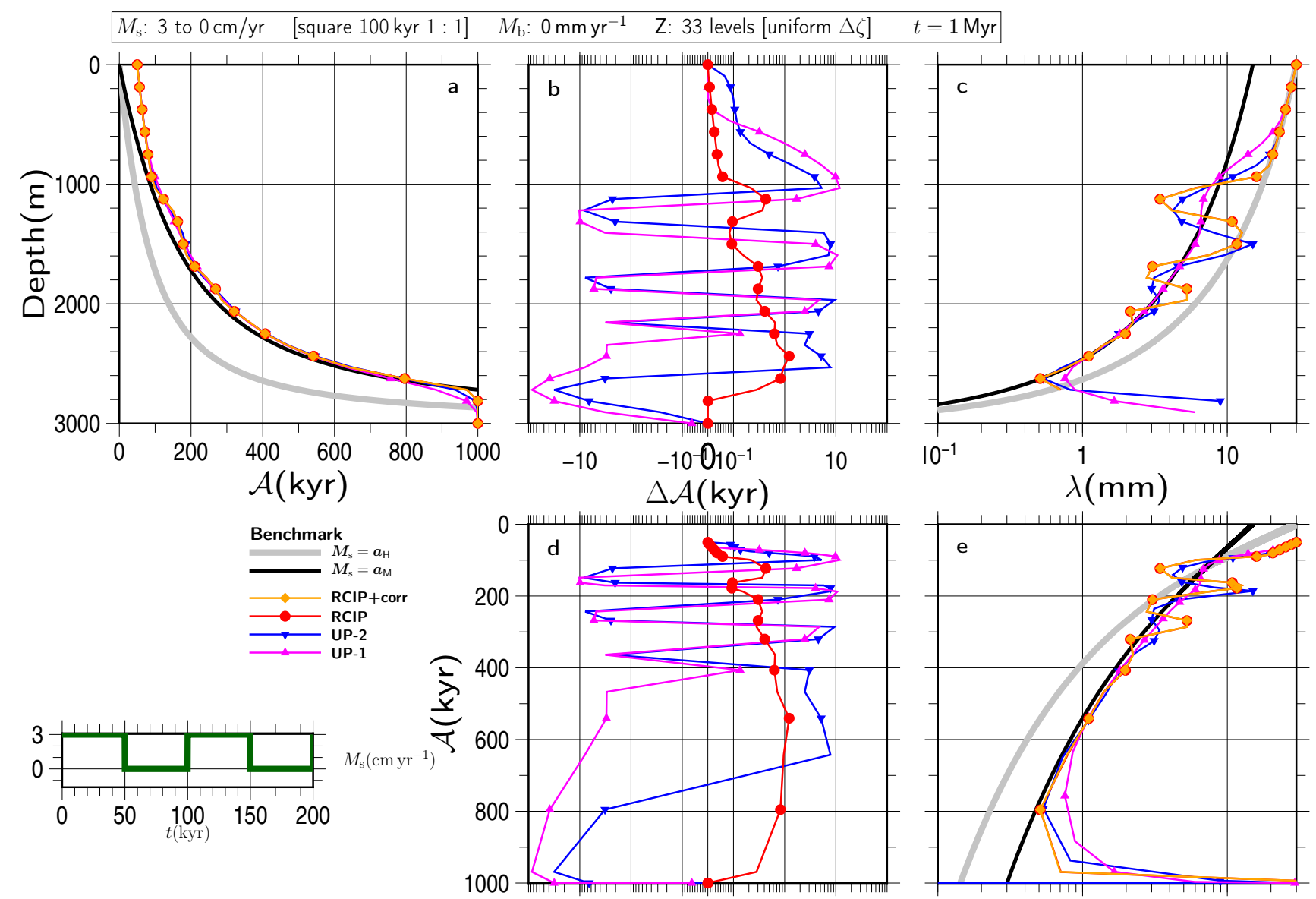

Figure S163
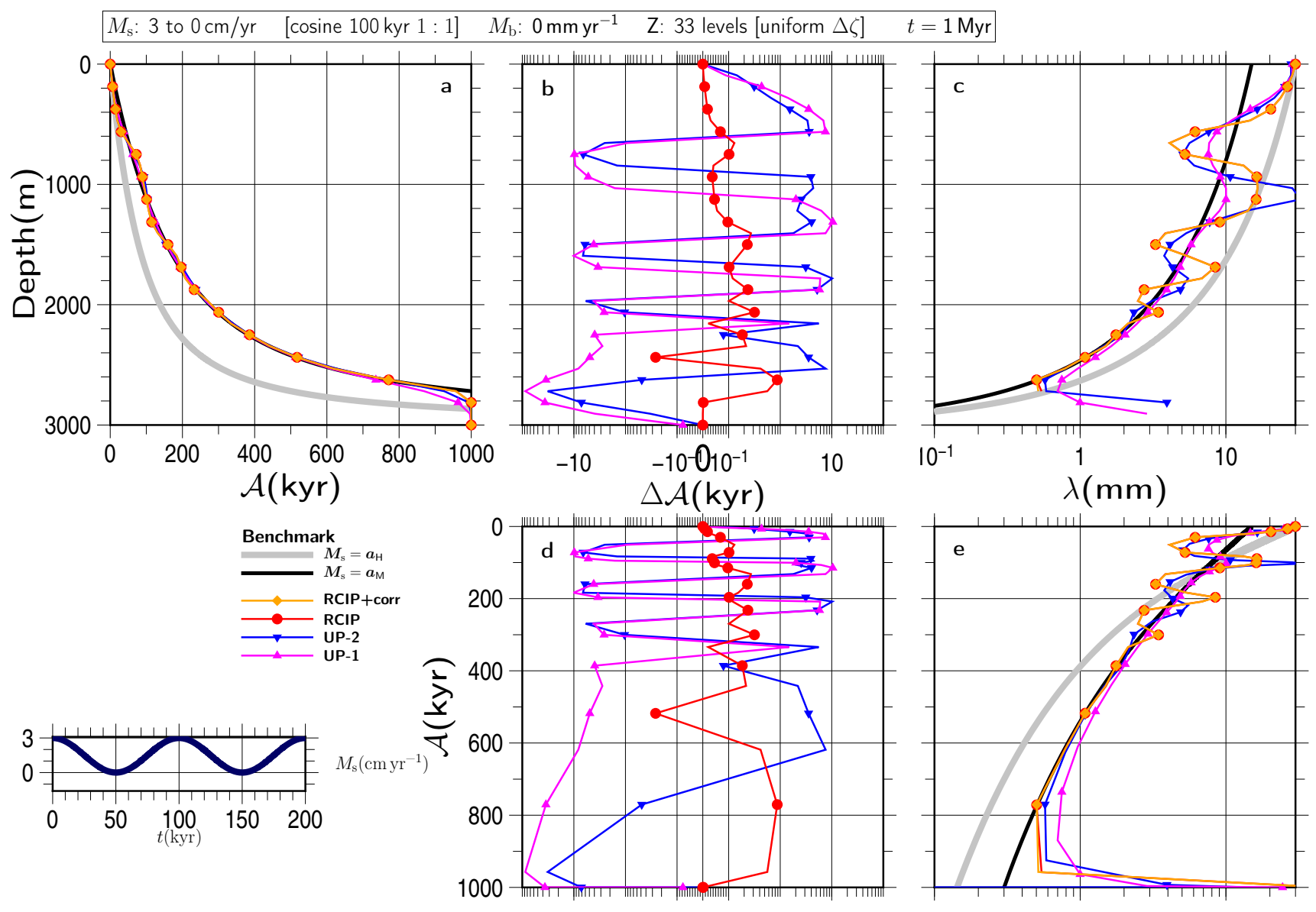

Figure S164 

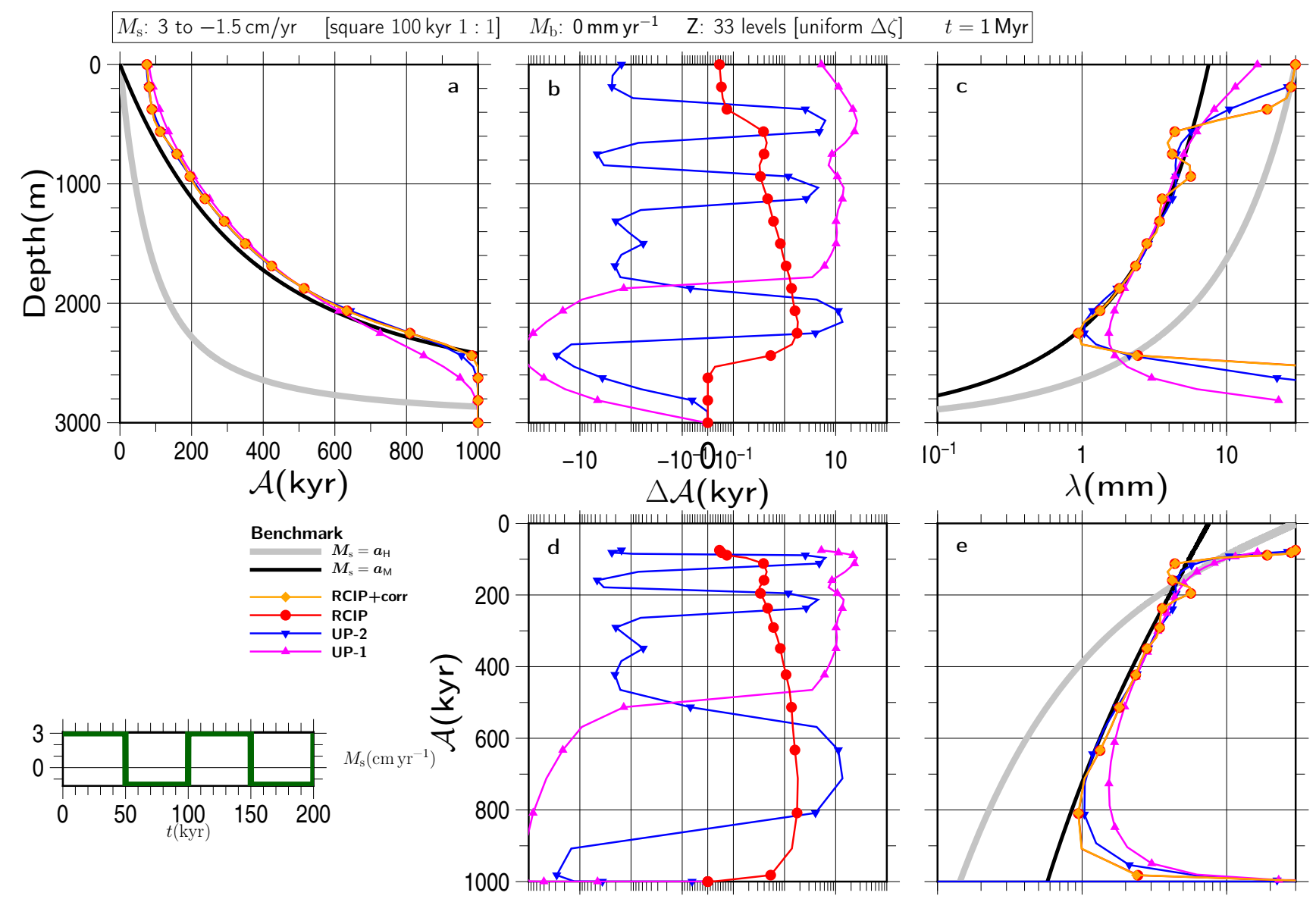

Figure S165
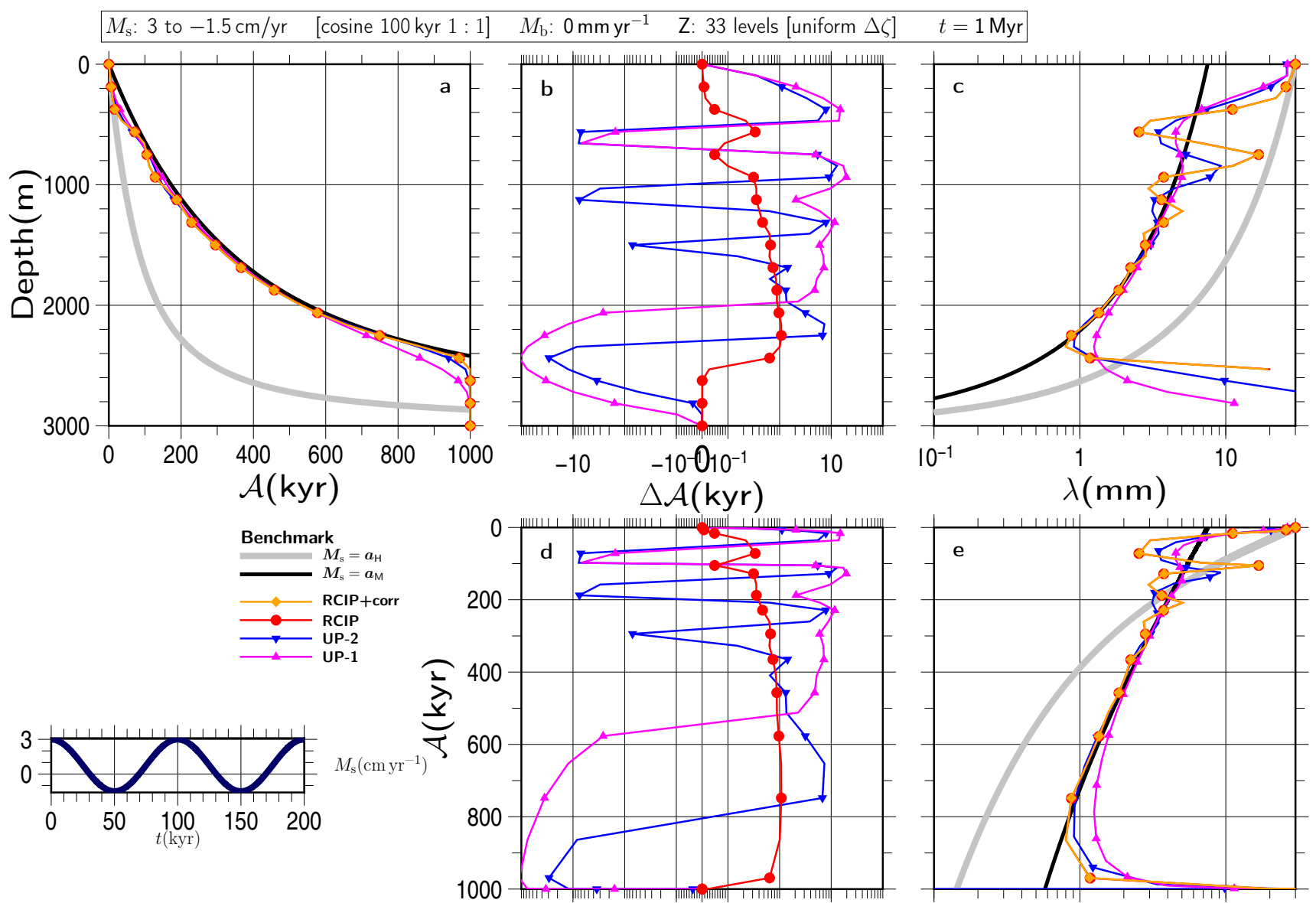

Figure S166 